\title{
Application of Value of Information to Tank Waste Characterization: A New Paradigm for Defining Tank Waste Characterization Requirements
}

L. L. Fassbender M. E. Brewster

A. J. Brothers

S. W. Gajewski

B. L. Harper

T. Eppel ${ }^{(a)}$

R. John ${ }^{(a)}$

November 1996
J. G. Hill

V. L. Hunter

D. A. Seaver

T. W. Wood

D. von Winterfeldt ${ }^{(a)}$

J. W. Ulvila ${ }^{(b)}$

Prepared for the U.S. Department of Energy under Contract DE-AC06-76RLO 1830

Pacific Northwest National Laboratory Richland, Washington 99352

(a) Decision Insights

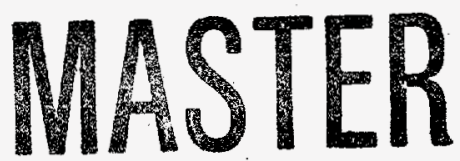

(b) Decision Science Associates, Inc. 


\section{DISCLAMMER}

Portions of this document may be illegible in electronic image products. Images are produced from the best available original document. 


\section{DISCLAIMER}

This report was prepared as an account of work sponsored by an agency of the United States Government. Neither the United States Government nor any agency thereof, nor any of their employees, make any warranty, express or implied, or assumes any legal liability or responsibility for the accuracy, completeness, or usefulness of any information, apparatus, product, or process disclosed, or represents that its use would not infringe privately owned rights. Reference herein to any specific commercial product, process, or service by trade name, trademark, manufacturer, or otherwise does not necessarily constitute or imply its endorsement, recommendation, or favoring by the United States Government or any agency thereof. The views and opinions of authors expressed herein do not necessarily state or reflect those of the United States Government or any agency thereof. 


\section{Executive Summary}

This report presents the rationale for adopting a recommended characterization strategy that uses a risk-based decision-making framework for managing the Tank Waste Characterization Program at Hanford. The risk-management/value-of-information (VOI) strategy that is illustrated explicitly links each information-gathering activity to its cost and provides a mechanism to ensure that characterization funds are spent where they can produce the largest reduction in risk. The approach was developed by tailoring well-known decision analysis techniques to specific tank waste characterization applications. This report illustrates how VOI calculations are performed and demonstrates that the VOI approach can definitely be used for real Tank Waste Remediation System (TWRS) characterization problems.

The goal of a characterization strategy is to provide timely information to support decisions. Information has value if it leads to better decisions, which are those with better expected consequences. Thus the VOI should be gauged by the expected increase in the value of decisions. Frequently, information will reduce uncertainty about consequences. Information will also often lead to decisions that involve reduced risks.

Qualitatively speaking, a source of information (e.g., a tank waste sample) is valuable if it has the potential for changing subsequent decisions, such as whether to keep transuranic (TRU) waste separate from high-level waste (HLW). For a source of information to be valuable, two conditions must be met:

1. The decision alternatives must have uncertain consequences (otherwise, there is nothing to be learned from the information).

2. Depending on the information obtained, different decisions may be best (otherwise, the information would not have any impact).

VOI decision analysis techniques are used for determining risk-based characterization requirements. This approach provides an understandable technical basis that explicitly links sampling, analysis, physicalchemical modeling, and other "learning" activities to risk reduction. Furthermore, VOI analysis provides a clear measure of completion because additional characterization activities are not justified when the costs exceed the calculated risk reduction value.

Risk-based requirements defined in terms of the VOI become a defensible basis for integration and prioritization of needs across tanks and program elements requiring information about waste composition, phenomenology, and performance. Knowing the value of obtaining specific information will provide an explicit basis for investments in research and/or technology development to reduce costs and technical uncertainty. This approach will also provide a defensible basis for budgeting and scheduling decisions by providing the means for selecting the most impactful characterization work for funding each year. This integration, coupled with statistically and technically sound sampling and analysis, monitoring, and laboratory methods, will result in an implementation plan that provides the most cost-effective characterization program. 
There are substantial costs associated with tank waste characterization activities, regardless of whether these activities involve detailed modeling and analysis from process flowsheets or sampling and laboratory analysis. These high costs provide an incentive to carefully estimate the VOI and to articulate a strong justification for spending resources on acquiring additional information. 


\section{Contents}

Executive Summary $\ldots \ldots \ldots \ldots \ldots \ldots \ldots \ldots \ldots \ldots \ldots \ldots \ldots \ldots \ldots \ldots$

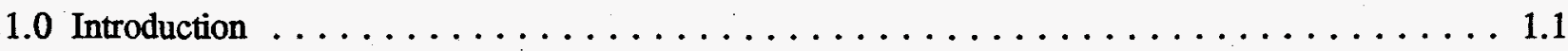

1.1 Purposes of the Report $\ldots \ldots \ldots \ldots \ldots \ldots \ldots \ldots \ldots \ldots \ldots \ldots \ldots \ldots \ldots$

1.2 Rationale for a Risk-Based Decision-Making Framework $\ldots \ldots \ldots \ldots \ldots \ldots$

1.3 Application and Limitations of the VOI Approach $\ldots \ldots \ldots \ldots \ldots \ldots$

2.0 The Recommended Strategy $\ldots \ldots \ldots \ldots \ldots \ldots \ldots \ldots \ldots \ldots \ldots \ldots \ldots \ldots$

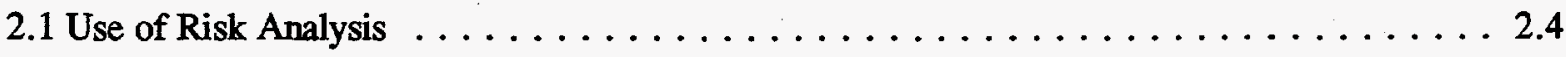

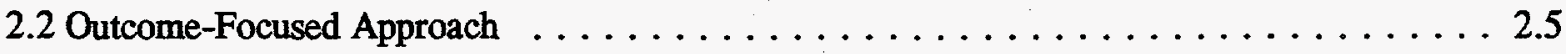

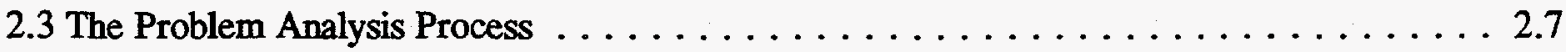

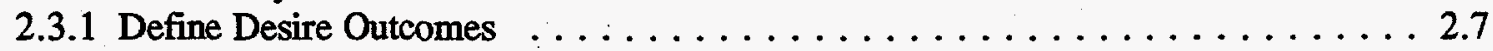

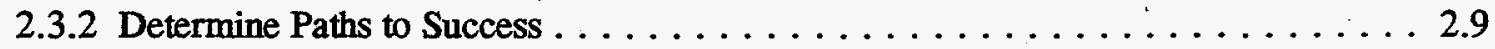

2.3 .3 Justify Information Needs . . . . . . . . . . . . . . . . 2.16

2.3.4 Use Waste Actions as Characterization Opportunities . . . . . . . . . 2.21

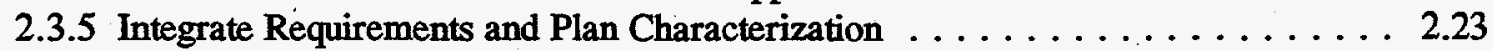

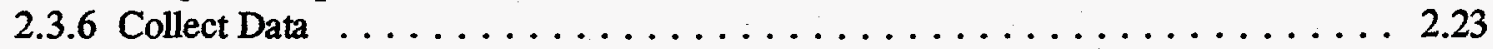

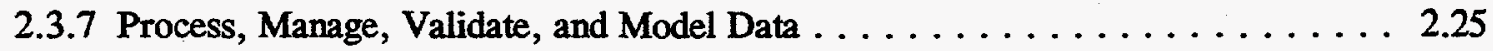

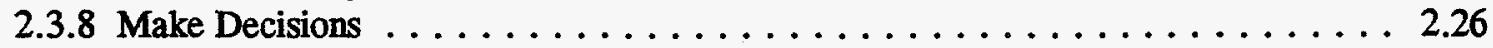

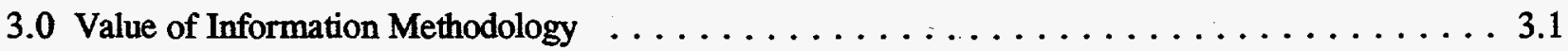

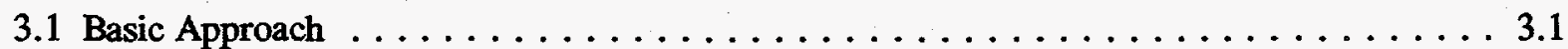

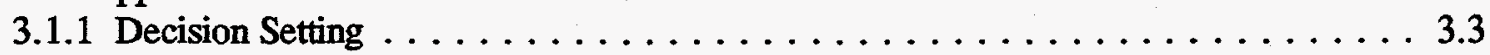

3.1 .2 Structure of the VOI Model $\ldots \ldots \ldots \ldots \ldots \ldots \ldots \ldots \ldots \ldots \ldots$

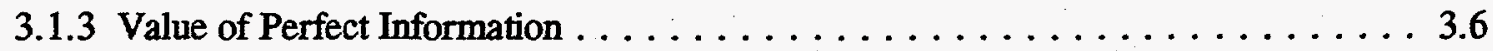

3.1.4 Value of Imperfect Information $\ldots \ldots \ldots \ldots \ldots \ldots \ldots \ldots \ldots \ldots \ldots \ldots \ldots$

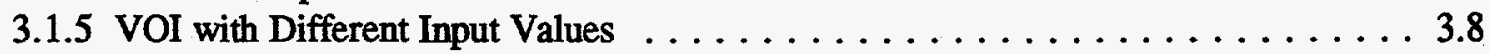

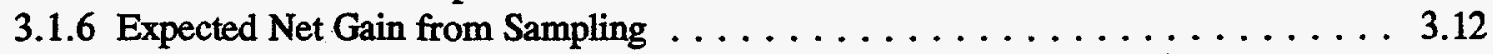

3.1.7 Estimation Approach: Introduction to Loss Functions . . . . . . . . . . . 3.13

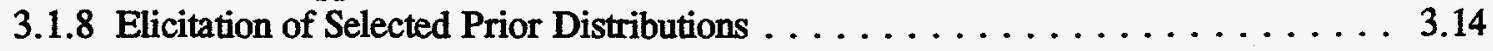

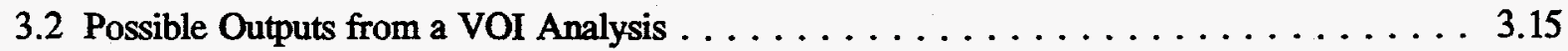

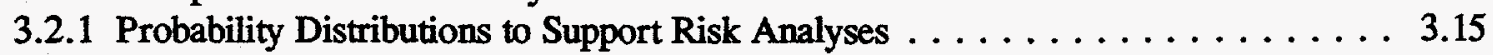

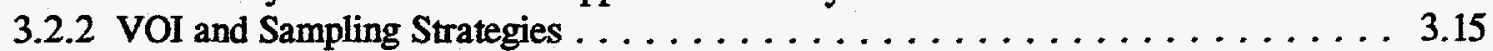

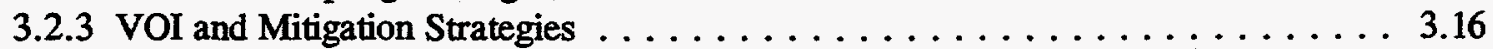

3.2 .4 Sequential Sampling Strategies $\ldots \ldots \ldots \ldots \ldots \ldots \ldots \ldots \ldots \ldots \ldots \ldots \ldots$

3.2.5 Informal Strategies Across Tanks . . . . . . . . . . . . . . 3.18 
3.2.6 Characterization Plans $\ldots \ldots \ldots \ldots \ldots \ldots \ldots \ldots \ldots \ldots \ldots \ldots . \ldots \ldots$

3.3 Incorporation of Risk-Based Constraints $\ldots \ldots \ldots \ldots \ldots \ldots \ldots \ldots \ldots \ldots .19$

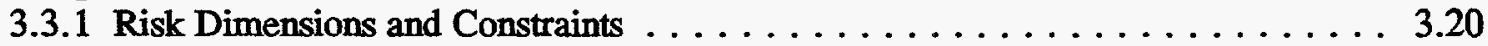

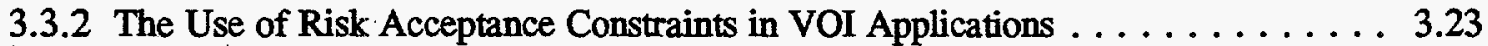

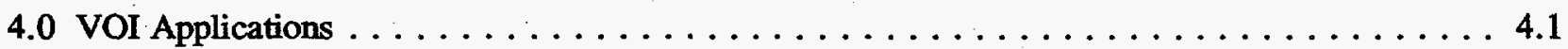

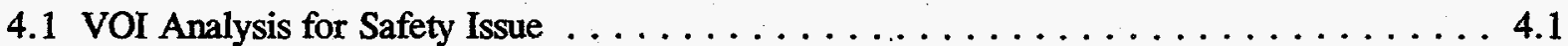

4.1.1 Organic Nitrate Problem Description $\ldots \ldots \ldots \ldots \ldots \ldots \ldots \ldots \ldots . . \ldots 4.1$

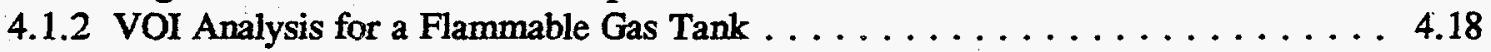

4.2 VoI Analysis for Disposal Decisions $\ldots \ldots \ldots \ldots \ldots \ldots \ldots \ldots \ldots \ldots .37$

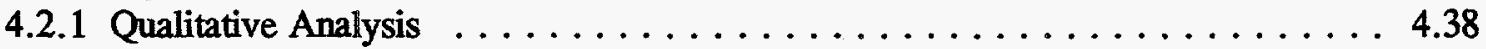

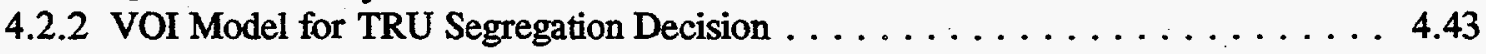

4.2.3 VOI Model for Technetium Feed Specification Decision . . . . . . . . 4.57

5.0 Basis for Integration of VOI and Prioritization of Requirements $\ldots \ldots \ldots \ldots \ldots \ldots .1$

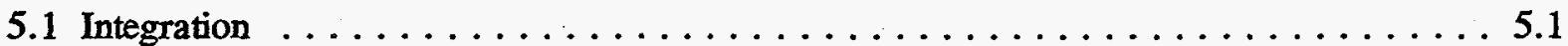

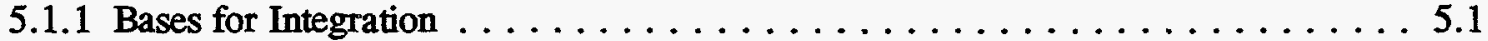

5.1 .2 Measuring Values on a Dollar Basis $\ldots \ldots \ldots \ldots \ldots \ldots \ldots \ldots \ldots .2$

5.2 Integrating Value of Information to Create a Characterization Strategy $\ldots \ldots \ldots \ldots .2$

5.2 .1 Types of Integration $\ldots \ldots \ldots \ldots \ldots \ldots \ldots \ldots \ldots \ldots \ldots \ldots \ldots \ldots \ldots \ldots \ldots \ldots .2$

5.2 .2 Basic Approach to Aggregation and Prioritization $\ldots \ldots \ldots \ldots \ldots \ldots .3$

5.2 .3 Additivity Issues and Approaches $\ldots \ldots \ldots \ldots \ldots \ldots \ldots \ldots \ldots \ldots \ldots \ldots \ldots$

5.3 Bounding Estimates for VOI $\ldots \ldots \ldots \ldots \ldots \ldots \ldots \ldots \ldots \ldots \ldots \ldots \ldots \ldots \ldots \ldots \ldots \ldots$

6.0 References $\ldots \ldots \ldots \ldots \ldots \ldots \ldots \ldots \ldots \ldots \ldots \ldots \ldots \ldots .6 .1$

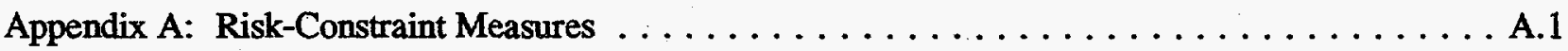

Appendix B: Condensed Phase Organic Reactions Safety Issue Information $\ldots \ldots \ldots \ldots \ldots$ B.1 


\section{Figures}

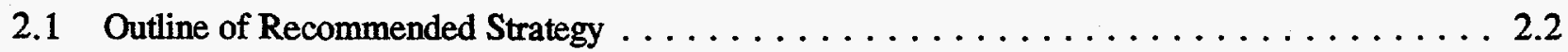

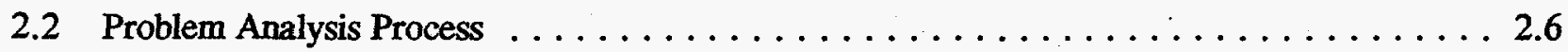

2.3 Participants in a Process for Defining Desired Outcomes $\ldots \ldots \ldots \ldots \ldots \ldots \ldots \ldots$

2.4 Underlying Thought Process for Preparing and Organizing Structured Logic Diagrams . . . . 2.10

2.5 Example of Preliminary Risk Evaluation $\ldots \ldots \ldots \ldots \ldots \ldots \ldots \ldots \ldots \ldots \ldots \ldots \ldots$

2.6 Example of Value of Information as a Function of Uncertainty $\ldots \ldots \ldots \ldots \ldots \ldots \ldots$

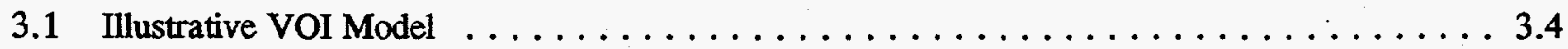

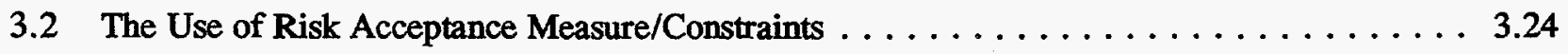

4.1 Decision Tree for the Organic-Nitrate Safety Issue $\ldots \ldots \ldots \ldots \ldots \ldots \ldots \ldots .7$

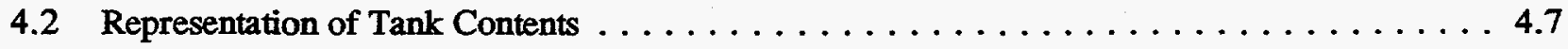

4.3 Expected Value of Perfect Information for the Organic-Nitrate Safety

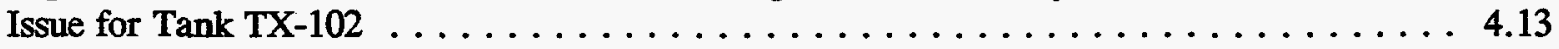

4.4 VOI Versus Quality of Information $\ldots \ldots \ldots \ldots \ldots \ldots \ldots \ldots \ldots \ldots \ldots \ldots \ldots \ldots \ldots \ldots \ldots$

4.5 Expected Value of Information (EVPI) Profile Condensed Phase Organic-Nitrate Reactions in Saltcake Tanks . . . . . . . . . . . . . . . . . . 4.16

4.6 Annual Frequency of Dome Collapse $\ldots \ldots \ldots \ldots \ldots \ldots \ldots \ldots \ldots \ldots \ldots \ldots \ldots \ldots \ldots \ldots \ldots \ldots .22$

4.7 Cumulative Distribution of Lifetime Dome Collapse Probability $\ldots \ldots \ldots \ldots \ldots \ldots .25$

4.8 Flammable Gas Safety Structured Logic Diagram $\ldots \ldots \ldots \ldots \ldots \ldots \ldots \ldots \ldots . .27$

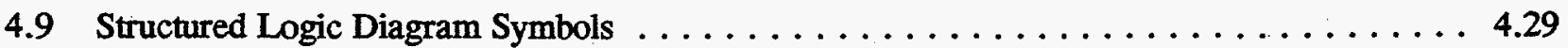

4.10 Sensitivity Analysis of Input Parameters $\ldots \ldots \ldots \ldots \ldots \ldots \ldots \ldots \ldots \ldots . . \ldots \ldots$

4.11 Decision Tree for TRU Segregation Problem $\ldots \ldots \ldots \ldots \ldots \ldots \ldots \ldots \ldots .45$

4.12 Sensitivity Analysis on Cost of Segregation and Penalty for Late Disposal . . . . . . . . 4.50

4.13 Sensitivity Analysis on the Probability that Tank 241-T-111 is Not Contaminated with HLW . 4.50 
4.14 Two-Way Sensitivity Analysis on $\mathrm{p}$ and $\mathrm{q} \ldots \ldots \ldots \ldots \ldots \ldots$

4.15 Influence Diagram for ${ }^{99} \mathrm{Tc}$ Characterization $\ldots \ldots \ldots \ldots \ldots \ldots$

4.16 Prior Distribution of ${ }^{99} \mathrm{Tc}, \mu \ldots \ldots \ldots \ldots \ldots \ldots \ldots \ldots$

4.17 Linear Loss Functions, $L(u, D)$ Where $u=22.5$ ) for Varying Values of the Optimist's

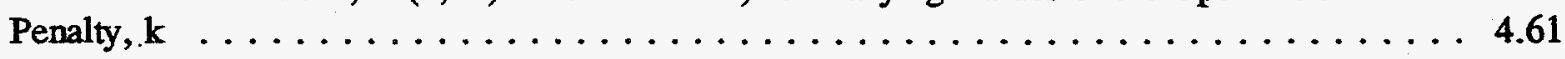

4.18 Quadratic Loss Functions for Varying Values of Optimist's Penalty, k . . . . . . . . . . . 4.62

4.19 Expected Loss as a Function of the ${ }^{90} \mathrm{Tc}$ Estimate $\ldots \ldots \ldots \ldots . \ldots \ldots$

4.20 Expected Loss as a Function of the ${ }^{99} \mathrm{Tc}$ Estimate $\ldots \ldots \ldots \ldots \ldots \ldots$

4.21 Expected Loss for Different Levels of Diagnosticity Compared with No Information Case . . 4.65

4.22 Expected Linear Loss as a Function of Optimists Penalty (k) and Diagnosticity of the

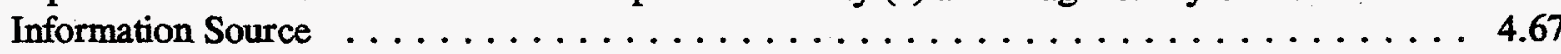

4.23 Expected Quadratic Loss as a Function of Optimists Penalty (k) and Diagnosticity of the Information Source 


\section{Tables}

3.1 Expected Net Gain from Sampling Analysis $\ldots \ldots \ldots \ldots \ldots \ldots \ldots \ldots \ldots \ldots \ldots \ldots$

3.2 Individual Risk Measures $\ldots \ldots \ldots \ldots \ldots \ldots \ldots \ldots \ldots \ldots \ldots \ldots \ldots \ldots \ldots \ldots \ldots \ldots \ldots \ldots .21$

4.1 Ignition Frequencies for Energy Sources $\ldots \ldots \ldots \ldots \ldots \ldots \ldots \ldots \ldots \ldots \ldots \ldots \ldots \ldots$

4.2 Estimated Probabilities of Ignition for Dry or Damp Reactive Waste $\ldots \ldots \ldots \ldots \ldots . .5$

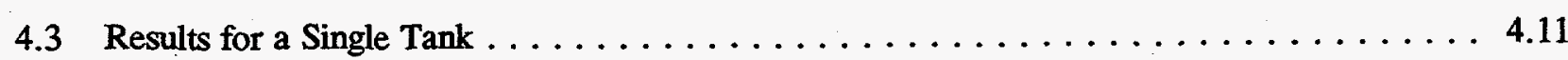

4.4 Summary Results for Tank $241-\mathrm{TX}-102 \ldots \ldots \ldots \ldots \ldots \ldots \ldots \ldots \ldots \ldots \ldots \ldots \ldots \ldots \ldots$

4.5 Risk Acceptance Criteria under Consideration $\ldots \ldots \ldots \ldots \ldots \ldots \ldots \ldots \ldots .20$

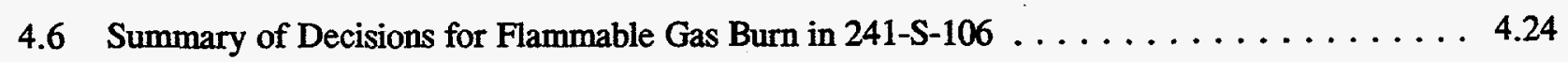

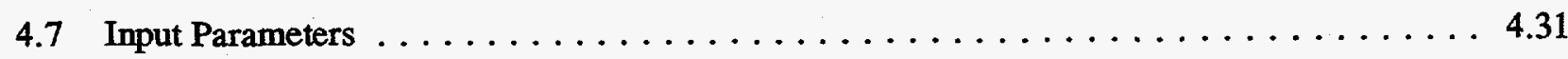

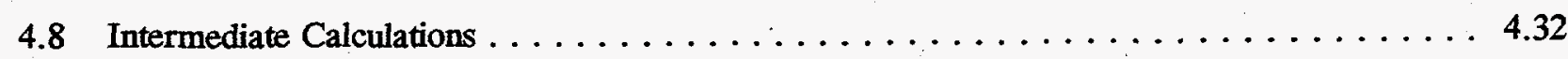

4.9 Qualitative VoI Analysis for Waste Retrieval and Processing Decisions . . . . . . . 4.40

4.10 Qualitative VOI Analysis for Specifications for Privatization $\ldots \ldots \ldots \ldots \ldots \ldots .4 .4$

4.11 Qualitative VOI Analysis for Pretreatment and Waste Form Decisions . . . . . . . 4.41

4.12 Qualitative VOI Analysis for Interim Storage and Transportation Decisions . . . . . . . 4.42

4.13 Qualitative Vor Analysis for Tank Closure and Final Disposal Decisions . . . . . . . 4.42

4.14 Probability Distributions for Glass Loading Factors $\ldots \ldots \ldots \ldots \ldots \ldots \ldots .47$

4.15 Probability Distributions for Cost $\ldots \ldots \ldots \ldots \ldots \ldots \ldots \ldots \ldots \ldots \ldots \ldots \ldots \ldots \ldots \ldots \ldots$

4.16 Expected Equivalent Cost for TRU Segregation Alternatives . . . . . . . . . 4.49

4.17 Expected Value of Perfect Information Analyses $\ldots \ldots \ldots \ldots \ldots \ldots \ldots \ldots . .52$

4.18 Diagnosticities for Imperfect Information $\ldots \ldots \ldots \ldots \ldots \ldots \ldots \ldots \ldots \ldots \ldots \ldots \ldots$

4.19 Application to all TRU Tanks $\ldots \ldots \ldots \ldots \ldots \ldots \ldots \ldots \ldots \ldots \ldots \ldots . . \ldots \ldots$

4.20 Preliminary VOI Results and Recommendations $\ldots \ldots \ldots \ldots \ldots \ldots \ldots \ldots . .55$ 


\subsection{Introduction}

Understanding the content and phenomenology of the waste in the underground storage tanks at Hanford is critical to the success of the Tank Waste Remediation System (TWRS) Program. The most immediate need for this knowledge is to ensure the safety of workers and the public by demonstrating that the contents of the tanks are stable and under control. Additional information is needed to proceed with the final disposal of the waste through processing and immobilization. For decision-making related to tank safety issues, data on tank chemical and radionuclide concentrations, moisture content, and temperature are needed to predict events that might occur and the effects of mitigation actions. For example, for disposal decision-making purposes, knowledge of whether tanks can be classified as transuranic (TRU) waste can help determine whether TRU should be segregated from high-level waste (HLW).

Characterization, broadly defined, provides the mechanism for obtaining this knowledge. All knowledge-development activities (including sampling and analysis, historical data analysis, modeling, and laboratory studies) are included in this definition. Current understanding of tank contents and phenomenology includes considerable uncertainty. In such an environment, a characterization strategy that is cost-effective and technically defensible is required.

\subsection{Purposes of the Report}

The purposes of this report are to

- present the rationale for adopting a risk-based decision-making framework for managing the Tank Waste Characterization Program

- describe an approach that was developed by tailoring well-known decision analysis techniques to specific applications for the Tank Waste Characterization Program

- illustrate how value of information (VOI) is calculated

- demonstrate that the VOI approach can be used for real tank waste characterization problems.

The risk-management/VOI framework illustrated in this report explicitly links each informationgathering activity to its cost and provides a mechanism to ensure that characterization funds are spent where they can produce the most risk reduction.

The report describes an approach to developing a characterization strategy based on the concept that characterization is intended to support making decisions and taking actions. In particular, the decisions and actions should effectively manage the risks associated with the tank waste. "Risk" is defined to include any uncertain and undesirable consequences of decisions, especially health and safety effects on workers and the public; effects on the environment and other natural and cultural resources; and effects on the cost, schedule, and technical performance of TWRS. 
This approach is based on decision analysis (Clemen 1991; von Winterfeldt and Edwards 1986), which analyzes decisions in terms of their potential consequences on multiple value dimensions (e.g., risk) and uncertainties in these consequences. In this framework, the goal of a characterization strategy is to provide timely information to support decisions. Information leads to better decisions, which lead to better expected consequences. Thus, the VOI should be gauged by the increase in the expected value of decisions. The VOI approach and applications described in this report provide an explicit assessment of the value of characterization information with respect to particular tank waste program decisions, which can then be used to develop a broad characterization strategy.

\subsection{Rationale for a Risk-Based Decision-Making Framework}

Any decision involving some chance of an adverse outcome is a risky decision. In this sense, almost all serious decision-making is risky, and "risky" need not mean carrying dire consequences. Risk-based decision making implies that we deal with what concerns us about a problem (i.e., our values), and that we deal explicitly with uncertainty and are willing to quantify it (i.e., estimate and use probabilities). Thus the only preconditions for risk are uncertainty and possible adversity to some decision maker.

In the context of the Hanford tanks, the relevant adverse outcomes are associated with management and remediation of the HLW they contain, including the following:

- Release of radionuclides to groundwater and subsequent human exposure.

- "Routine" radiological dose to tank farm workers due to contamination of the tank farms over time and/or associated with in-tank operations.

- Human exposure due to uncontrolled release of radionuclides or toxic constituents from the tanks as a result of a fire, explosion, or criticality in the waste itself, or burning/detonation of gases in the tank headspace.

- A large class of actions in which there is failure to appropriately manage the waste in the long term, including inadequate retrieval, ineffective waste treatment after retrieval, or simply the prospect of spending much more time and tax dollars than necessary in the process of waste management, remediation, and disposal.

These are different kinds of risks, measured in different ways, and they have different levels of importance to different stakeholders. What many of these risks have in common, however, is some degree of dependence on the physical and chemical conditions in the waste tanks. This, and the fact that these conditions are only partially known, is the basic rationale for any characterization program.

Tank waste constituent or behavioral knowledge should have no special stature in the risk assessment process. Since many types of factors influence estimated risk, reducing uncertainty in any of them can be beneficial. Whether it is smart to spend millions on obtaining better tank waste data depends on the other opportunities for reducing uncertainty, and the effects and cost of these options as well. 
It is important that these uncertainties are included in the risk analysis for a particular decision, given that the basic decision-making framework is known with confidence. This framework includes the timing of the decision and its implementation, the available options, their costs, their effects-in short, a description of the decision that is sufficient to actually make it with some degree of confidence.

Since a risk estimate is based on estimates for key measurable parameters in the tank, including our current uncertainty about them, the option to get more information can be represented by alternative sampling schemes (or other analysis, modeling, etc.), each of which has an expectation for uncertainty (and thus estimated risk) reduction. Many people instinctively rebel at the notion that information gathering can really reduce risk because it does not change the real conditions in the tank. This objection overlooks two key points. First, decisions are always made based on estimated risks. We never know "real" risks. Second, the risk-reduction value of better information about tank conditions comes about by virtue of better decisions about the things which do change the real conditions. With better information about fuel, moisture, initiator frequencies, and the like, we will make fewer overly conservative decisions to mitigate, and fewer inappropriately risky decisions to do nothing. So in an ex-ante sense, the information actually does reduce risk.

The kind of analysis briefly described here is a powerful but demanding framework. It requires substantial investment in management and engineering time by those who own the problem. This model is in significant contrast to those that tend to separate the decisions about what information to get from those that deal with what to do in the mitigative domain. It forces decision makers to push their knowledge and be candid about its limitations. It requires, most fundamentally, that one is willing to admit the possibility of significant adverse outcomes and to quantify this possibility, since avoiding these outcomes is the only real incentive to learn more.

Such a risk-based framework has some clear advantages. First, it sets clear "completion conditions" for the characterization of any tank with respect to any issue or set of decisions. When the expected value of information does not exceed its expected cost, we are done. In a trial application of this method to the organic-nitrate reaction for single-shell tanks (SSTs) (Wood et al. 1995), even perfect (i.e., without any statistical uncertainty) information about fuel concentrations did not improve decision making enough to "pay the costs" of minimal sampling in many tanks. Since we will never derive anything approaching perfect information from real sampling and analysis, this quickly rules out large areas of characterization activity as effective risk-management tools. In contrast, the method makes the benefit of some characterization activities very obvious.

A second major advantage of this framework is that it places a distinct burden of proof on those who want better information to show just how it will provide some benefit. The result is that characterization activities are forced to compete for resources with projects that would take mitigative or treatment actions, which is as it should be.

Tank waste management is inescapably risky because it requires several very consequential decisions be made under significant uncertainty. A risk-based framework for planning characterization can be constructed with a clear logical structure in which risk-reduction value can be input to better information in 
several uncertainty domains, including tank waste inventories and physical-chemical phenomenology. Such a framework requires investment in formulating tank waste management decisions and in predicting several types of risks as functions of alternative actions and levels of uncertainty. It is sensible to talk about such a framework only if the overall management of the wastes is conducted in risk-management terms.

To the extent that a risk-management/VOI framework can be used to define requirements for TWRS characterization, there will be an understandable technical basis that explicitly links sampling, waste analysis, physical-chemical modeling, and other "learning" activities to risk reduction. This technical basis can tell us, in some detail, how to go about the tank waste characterization mission and when we have succeeded. It also explicitly links information gathering (and thus risk reduction) to its cost (and the costs of decisions without additional information). This framework then provides a mechanism to ensure that money is spent where it can produce the most risk reduction, an important feature in today's highly constrained funding environment.

\subsection{Application and Limitations of the VOI Approach}

While the application of the VOI approach will provide a sound technical basis for characterization strategy, some caveats do apply. These do not diminish the desirability of the approach, but they do need to be understood to ensure its credibility and appropriate use.

First, a VOI analysis is performed for a specific decision, such as those noted above. The number of decisions that could potentially be affected by better characterization information is large, and full VOI analysis for all such decisions may not be practical. A better approach may be to explicitly analyze key decisions (e.g., resolution of all safety issues and a few key disposal decisions) and use a qualitative approach such as that described in Section 4.2.1 to get a qualitative understanding of the value of characterization information for other decisions.

Second, much of the direct input to a VOI analysis comes from the judgments of technical experts. Ideally, these judgments are based on sound technical data and analysis and are the best source of integrated information available. Systematic processes for capturing these judgments to minimize biases and maximize validity have been developed over many years and should be used.

Third, development of a full characterization strategy defining which tanks will be sampled when, and analyzed for which constituents; and what laboratory experiments, historical data analysis, and modeling will be done requires integration and generalization from the VOI analyses for individual decisions. For example, the desirability of one or more core samples from a particular tank could be based on how that information would potentially affect several decisions involving several different waste constituents. The methodological issues in such integration are not trivial but can be handled, as is discussed in Section 5.0, if the issues are understood and considered.

Fourth, the VOI analysis by itself will not demonstrate compliance with existing risk constraints such as those defined by law or regulation. Rather, the analysis will suggest the preferred alternative (and the 
VOI relative to that decision) based on some set of values, which, for the characterization strategy, includes some measures of risk. The compliance of this preferred alternative with such risk constraints will still need to be demonstrated. This issue is addressed in Section 3.3.

Section 2.0 provides an overview of the recommended characterization strategy, and Section 3.0 describes the VOI methodology. The basic VOI approach is illustrated with a complete, simplified VOI model. The structure, the required inputs, the calculations, and the outputs are described. The section also demonstrates how the VOI changes as the state of knowledge changes prior to gathering the information, changes in the costs of errors, and changes in the quality of information (i.e., its diagnosticity).

Section 4.0 provides applications for three specific decisions: 1) how to deal with organic nitrates (a safety issue), 2) whether to segregate TRU waste from HLW for processing and disposal, and 3) what the technetium specification should be for the low-level waste (LLW) feed to private vendors for immobilization. These three decision analysis applications demonstrate how the VOI methodology can be applied and provide insights into characterization needs for the specific decisions. Section 5.0 addresses how quantitative VOI analysis results from specific tank waste management decisions can be integrated to plan a costeffective characterization program. 


\subsection{The Recommended Strategy}

The details of the recommended strategy are described in this section, which focuses on the Hanford waste tank problem analysis process. Descriptions of each process step are prefaced by sections that discuss the strategy's underlying concept of risk, the use of risk analysis, and the need for risk-based management; the risk-based and outcome-focused nature of the strategy; and the waste characterization efforts that include taking advantage of routine waste operations and processing activities to derive characterization information.

Figure 2.1 shows a generalized outline of the recommended strategy, in which the work flow is as follows:

- The objective (i.e., resolve a safety problem) is identified, the desired outcome is specified, and resolution criteria for meeting the objective are defined.

- A Resolution Team is assembled and conducts an initial analysis of the problem using existing information (i.e., the current understanding of the waste properties and the physics and chemistry controlling the resolution of the problem of interest). A quality review ensures that data quality represents the needs of the Resolution Team.

- If the initial analysis finds that the resolution criteria are met (globally for the issue at hand or on a tank-by-tank basis), the desired outcome has been achieved.

- If the initial analysis finds that the resolution criteria are not met, actions are taken in a series of iterative steps until problem analysis shows that the resolution criteria are met through an improved understanding of the waste chemistry or physical condition or by mitigation through changing the waste properties or adding operational controls.

The success of this characterization strategy depends heavily on the decisions of the Resolution Team, who are key in managing the application of the recommended strategy. This team must be certain that the strategy is applied with the appropriate level of rigor to lead to timely and cost-effective actions. The team must eventually recommend to decision makers the actions that will lead to the accomplishment of the defined objectives. Success of the Resolution Team requires mechanisms for

- ensuring that all relevant information is addressed by the team

- managing the influence of business, programmatic, and other nontechnical considerations on decisions (appointing team members who do not have a vested interest in the outcome of the decision analysis might be difficult if the team members are engineers and scientists with a good understanding of the problems). 


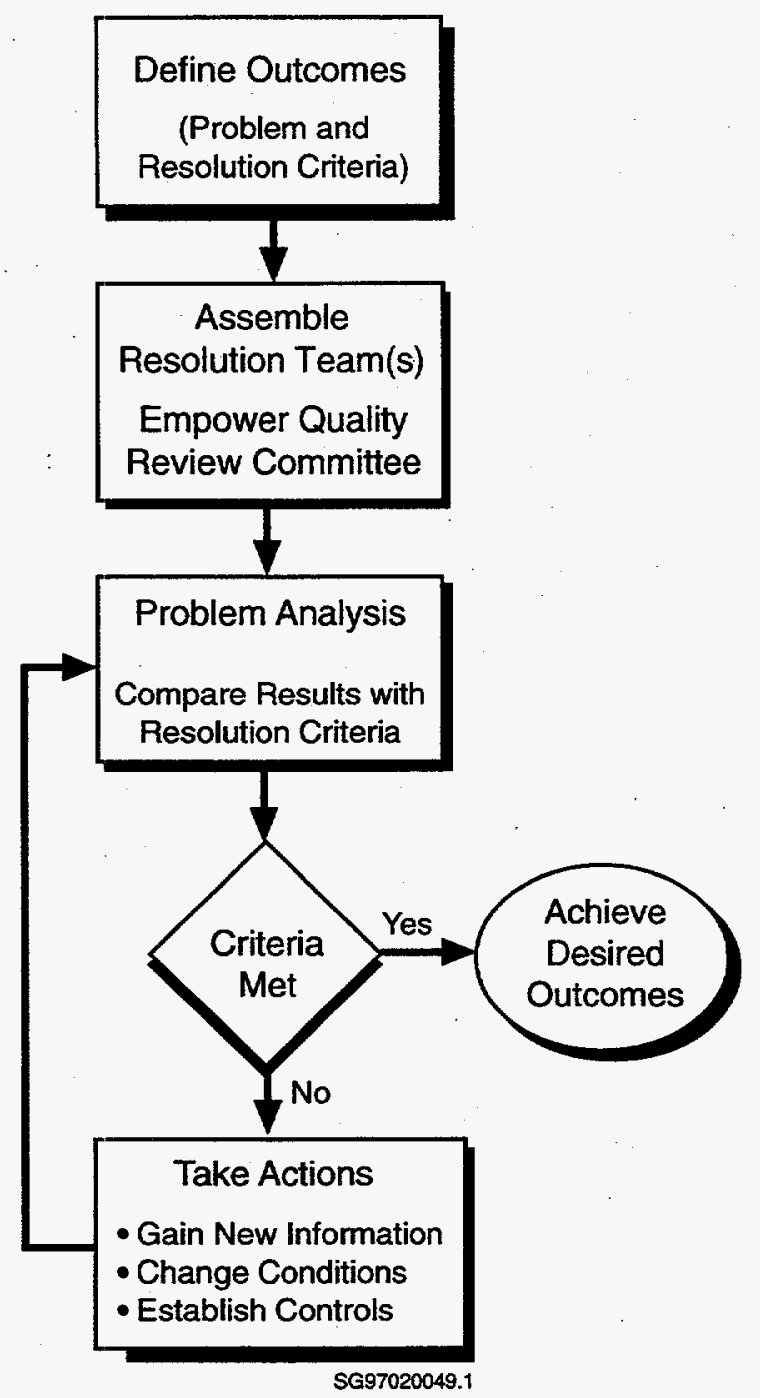

Figure 2.1. Outline of the Recommended Strategy

An approach used in other venues can successfully respond to these needs and concerns by providing appropriate checks and balances. The key features of this approach are as follows:

- The team is composed of technical staff who have the appropriate expertise to address the issue under consideration. These staff can be from DOE contractors, universities, national laboratories, and industry.

- The leadership and many team members may come from one contractor, but, to avoid conflict of interest, that contractor should not be responsible for most of the implementation work.

- The team members are drawn from the broad scientific and technical community, as needed. 
- A review process is implemented to ensure that the team's decisions are technically sound, respond to the need for implementable, timely solutions, and do not involve any conflict of interest.

The Resolution Team is expected to work full time if necessary until it has established a sound technical basis for resolution of the issue.

Establishment of a standing Quality Review Committee composed of highly qualified personnel from the national laboratories, industry, and academia is proposed as part of the recommended strategy. This committee would ensure that a data quality assurance program is in place and that it provides a scientifically justified and rigorous technical assessment of all data requests and results. The fundamental criterion for judging data quality is that the data reliably respond to the data needs specified by the Resolution Team.

The proposed basis for organizing the Quality Review Committee would include the following components:

- Membership is by appointment and based on technical qualifications and freedom from conflict of interest.

- Representative(s) of any party fiscally involved in an action are disqualified from making decisions benefiting their institution.

- Expert subcommittees may exist for select topics.

- The Quality Review Committee acts promptly if an urgent and unexpected technical issue arises in between regular reviews.

The Hanford Tank Waste Advisory Panel is an example of an existing review panel that could be changed to provide the Quality Review Committee function.

In summary, the recommended strategy calls for the establishment of three types of teams/committees/ panels to accomplish the work:

- Resolution Teams. These are contractor staff, augmented by others, who are responsible for identifying the details of the strategy(ies) and data needs required to resolve Hanford's tank characterization and interim-stage safety problems. The contractor primarily responsible for this part of the work may be the operations contractor, some other contractor, or a national laboratory. The key points are that the teams must work without conflict of interest and the team members must have appropriate qualifications for the work. Some problems will require the contractor to build upon its core staff with subcontractors from universities, industry, the national laboratories, etc.

- Quality Review Committee. This committee is to ensure that the data obtained through sampling and analysis of wastes will respond reliably to the data needs specified by the Resolution Teams. For 
example, it is expected that this committee will identify an alternative to the measurement of total organic carbon (TOC), which is only a weak indicator of the contribution of organic materials to the energy content of the waste.

- Review Panels. These can be standing or ad hoc panels that are to review the quality of the work, the capabilities/appropriateness of the key staff, and the work environment (e.g., evaluating whether Resolution Teams have sufficient authority and stability to be successful). This is not a technical advisory function. If those responsible for the work are in need of significant technical advice, the

Resolution Teams should be strengthened such that DOE and the public can be assured of the technical competence of the work.

This approach represents a clarification and simplification of current practices. Resolution Teams already exist by definition-they are the individuals working to address particular issues or concerns. However, they may not meet the criteria described previously. The Quality Review Committee and Peer Review Panels would replace existing advisory panels, separating their roles of helping to solve technical problems and reviewing progress such that one body is not reviewing actions that they themselves have directly or indirectly encouraged or planned.

\subsection{Use of Risk Analysis}

Before the strategy is described, it is important to discuss how the concept of risk is used in two different ways in this strategy. First, risk is used to define the acceptance criteria for the various elements (e.g., safe interim storage) of the waste storage and disposal mission (Harper et al. 1996). Risk includes both the probability and the consequences of an unwanted event taking place. For this application, the criteria need to respond to health and environmental protection needs as well as to programmatic concerns about the effect of an incident on the waste disposal mission. Limits of time and resources prevent the acceptable risk criteria from being set at arbitrarily low levels. In working with regulators and stakeholders to set these criteria, DOE is bound by the constraints of physical and fiscal reality. Establishing these bounds defines an important role for characterization work.

Risk is also used to guide decisions about taking the actions needed to achieve the waste storage and disposal mission. Not only must the results of selected actions (i.e., waste retrieval, transport, blending, processing) aid in achieving the criteria for success of the mission, but the actions themselves involve risk. Quantification of the risk associated with taking a particular action on the waste requires knowledge of the waste contents and properties as provided by waste characterization work. Using risk to select actions is discussed in Colson et al. (1996, Appendix B), where it is noted that, while it is important to stay below acceptable risk limits, taking the actions of lowest risk may not be the best decision. Some riskier actions might be justified if the data/information gained are expected to have sufficiently large beneficial effects.

The successful implementation of the recommended strategy requires that safety and waste disposal activities also be managed in a risk-based framework. The first and most basic requirement for management systems is that risk-management principles be used consistently across all related programs. This requirement means that the management of safety and waste disposal functions acknowledges that achiev- 
ing zero risk is not possible. In tank safety management, we cannot maintain that "there are no significant risks but we just cannot quite prove it yet." Risk must be acknowledged to gauge the incentive(s) to conduct appropriate characterization activities. The level of acceptable risk can be made as small as is affordable, but a position that "any risk is too much" cannot be realistically maintained.

In the realm of waste retrieval and processing, risk-based management allows for some tolerable degree of uncertainty in process development and application. A workable reprocessing technology at Hanford would never have been developed if a requirement had been imposed that the first process successfully used (bismuth phosphate) be perfectly efficient. The degree of uncertainty permitted need not be large or reflect a permanent end state, but it should allow for enough flexibility to actually do some waste processing and to learn while doing so. The best technical solution will emerge from experience. Learning takes practice, and practice requires that decisions be made and actions be undertaken. There is no substitute for the experience that comes from actually dealing with the tank waste within constraints that limit risks to acceptable levels.

\subsection{Outcome-Focused Approach}

The characterization effort must be intimately tied and integrated into the work required to achieve specific waste management objectives. It focuses on achieving the specific set of outcomes that rely upon understanding the waste and related systems. Characterization work seeks to answer questions such as, Do the properties of the waste represent a potential energy release hazard? and, How much risk is associated with that energy release? Characterization, safety/risk analysis, decision making, and large-scale remedial actions applicable to tanks are all part of the same interrelated and interactive system (Figure 2.2) in which justification for new data, characterization actions, validation/refinement of the model(s), and risk and decision analyses take place. This contrast with the current situation in which the characterization and safety programs are managed by multiple Hanford contractors, making coordination and integration difficult.

In some instances, all steps shown in Figure 2.2 (from defining desired outcomes to taking actions) are exercised as the recommended process is worked. For other iterations, only select steps are used based on specific data/information needs of analyses or option comparisons required by decision makers. In this fashion, flexibility is built into the recommended strategy as it is tailored to address the problem(s) at hand. This approach forms the basis for an integrated and effective characterization strategy. Ongoing characterization work cannot be considered complete until the final acceptable end state of any given tank has been achieved. At each iteration, the need for additional characterization work must be justified. The recommended strategy uses risk analysis as a basis for justifying data needs. In fact, one of the key strengths of a risk-based strategy is that the criteria provide a quantitative basis to break the collect-data/ model/collect-data cycle that is otherwise difficult to complete. When is the amount of information enough? It is enough when the cost of obtaining additional information exceeds the cost of remediation or control actions needed to reduce risks to sufficiently low levels. 


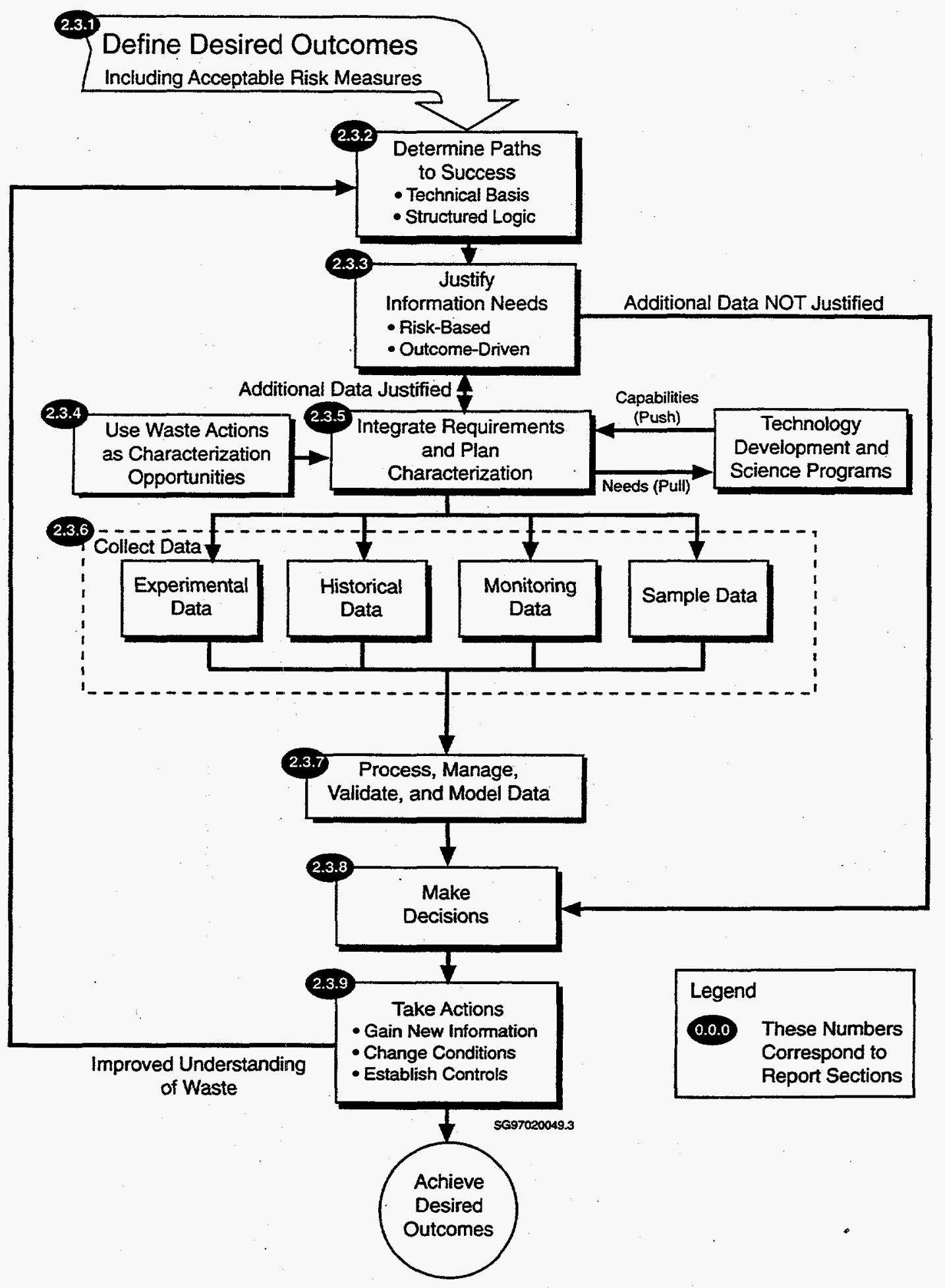

Figure 2.2. Problem Analysis Process 
Waste characterization, defined as obtaining the physical and/or chemical intelligence that provides enough information to allow actions to be taken to achieve a specified level of risk, will enable the quickest and most cost-effective, task-by-task (or tank-by-tank) closure of characterization work.

\subsection{The Problem Analysis Process}

The problem analysis process is shown in Figure 2.2 and described in Sections 2.3.1 through 2.3.9. The strategy employs structured logic diagrams to provide a graphic representation of the model(s) used to describe the phenomena associated with the disruptive event that is the basis of the safety issue. Structured logic tools guide development of an understanding of the problem. Decision analysis tools are then used to make risk-based decisions based upon this understanding. These problem-solving tools are developed and used to the degree of complexity that matches the complexity of the problem at hand. Using the tools does not guarantee success, but, when used by a team that understands the limitations of the available information and analysis methods, the likelihood of success is increased.

\subsubsection{Define Desired Outcomes}

Defining the desired outcomes (i.e., selecting a level of risk that is acceptable and that is believed to be physically and financially attainable) is the role of decision makers. Discussions are ongoing about what constitutes resolution of a safety issue. For example, concerns about minimizing risks can lead to the desire for no unexpected event to occur within a tank, even an event that would be too small to cause worker or public harm. Obviously, costs and other factors will be strongly affected by the level of risk that is deemed acceptable. Definition of these desired outcomes, including acceptable risk and risk uncertainty, is based on current understanding of the technical requirements underlying resolution of the safety issue, augmented by input about preferences and values of stakeholders (oversight panels, Native American Tribes, the public), regulators (federal and state), and national policies. The definition process is illustrated in Figure 2.3.

As shown in the figure, decisions about the 177 waste storage tanks at Hanford are a subset of the full range of DOE decision making, ranging from concerns about safety issues to other issues requiring resolution during the restoration of the Hanford Site. The needs and requirements of all sources are assimilated into desired outcomes. Either formally or informally, desired outcomes are defined for all waste management issues, including safe interim storage and waste disposal. In some cases, a general consensus has been forged (i.e., the disposition of the Hanford tanks as described in the Tri-Party Agreement between the DOE, U.S. Environmental Protection Agency [EPA] and the State of Washington) (Ecology 1996) and in others, such as land end states for the Hanford Site, the development of consensus is ongoing. A recommended, integral part of this process is early and open sharing of information and ideas among the project teams, DOE, regulators, stakeholders, tribes, and the public. This is often an iterative process as information about risks and the costs of managing risks is refined. Application of the recommended strategy will play an important role in this process by providing decision makers with the required technical information such as the current level of risk associated with each tank and the costs and tradeoffs associated with reducing that risk and its uncertainty. 


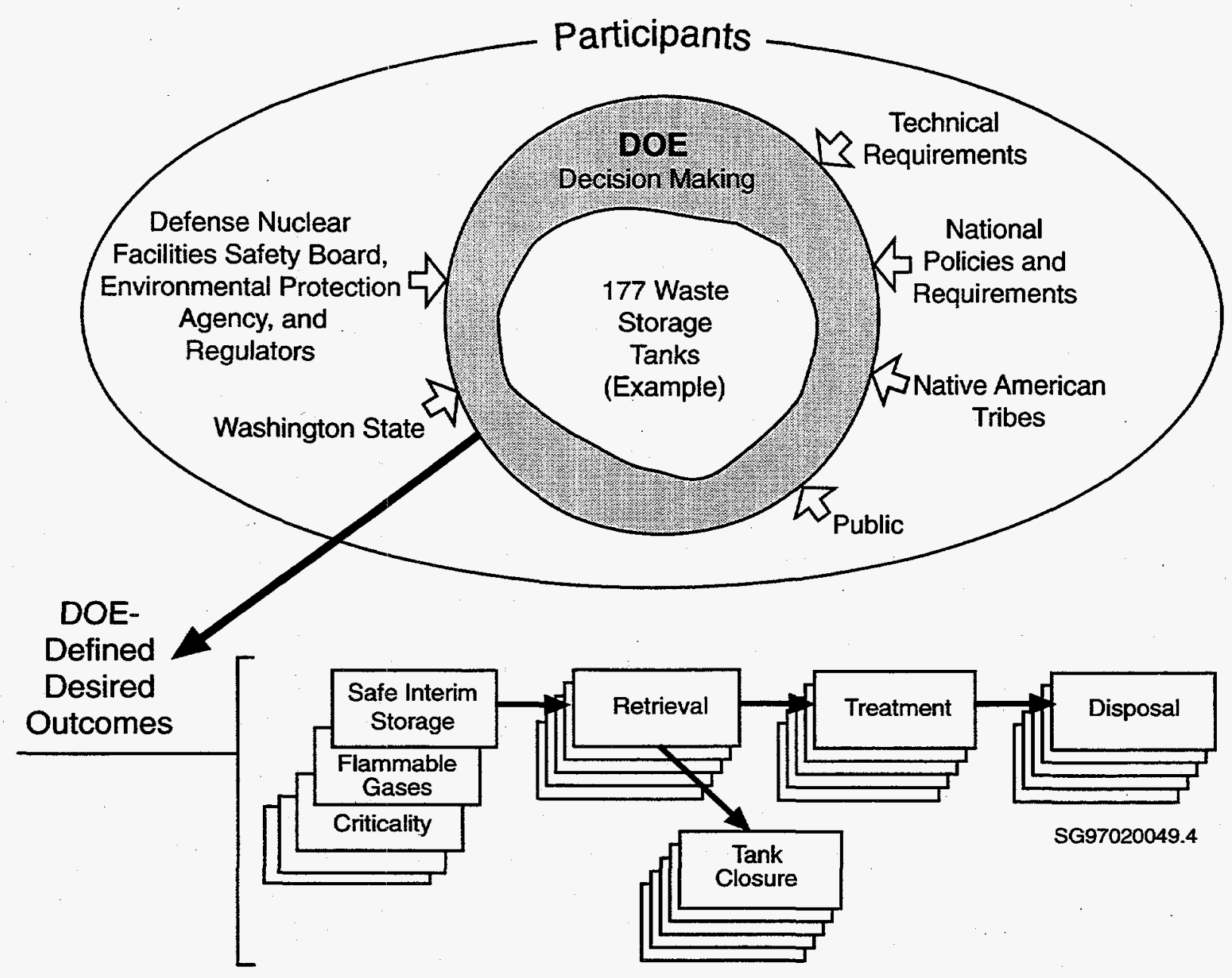

Figure 2.3. Participants in a Process for Defining Desired Outcomes

Not all tanks pose the same level of risk from a particular phenomenon; for example, an organic complexant fire is highly incredible in a tank that is mostly water. Nor are the risks from particular hazards separable. A flammable gas fire might ignite dry flammable solids. The strategy calls for the management of all tanks to a level of acceptable risk when integrated over all credible hazards. Some tanks may pass this test with ease, while others may require considerable study and modification of the tank contents or installation of operational controls to meet the acceptance criteria.

Acceptable risk and uncertainty must be developed into a measurable and appropriate set of risk attributes that contain technical and programmatic risks. Risk constraints and the methodologies to develop meaningful risk constraint levels are further detailed by Harper et al. (1996). As the understanding of the safety problem evolves and the level of available funding is specified, planning will account for what can realistically be accomplished. The technical feasibility and clarity of the desired outcomes and acceptance criteria will have a major effect on any strategy designed for managing tank safety risks. 


\subsubsection{Determine Paths to Success}

To achieve specific tank safety and remediation objectives (desired outcomes), the recommended strategy calls for the Resolution Team to manage the development of models that show

- alternative and technically sound solutions (paths to success) leading to each objective

- physical and chemical conditions that must exist to achieve those solutions

- information needed to assess the probability that each of these required conditions can be met.

A technical analysis approach called structured logic is a well-established tool for documenting the options for resolution of a problem and its associated information needs. Structured logic was found to be well suited to analysis of the tank waste safety issues. The results of this approach are contained in a structured logic diagram. The basic factors considered in preparing the structured logic diagram are illustrated in Figure 2.4. Such analysis of a problem generally leads to a variety of technically feasible options for achieving the desired outcomes. At the very least, the options include resolution through understanding, mitigation, and/or addition of controls. There may be several options within each of these categories. Structured logic provides a formal and detailed approach to guide the development of the technical basis for achieving specific desired outcomes. Structured logic diagrams provide concise documentation of the desired outcome, definition of alternative success paths, definition of data needs, and modeling requirements or data processing requirements.

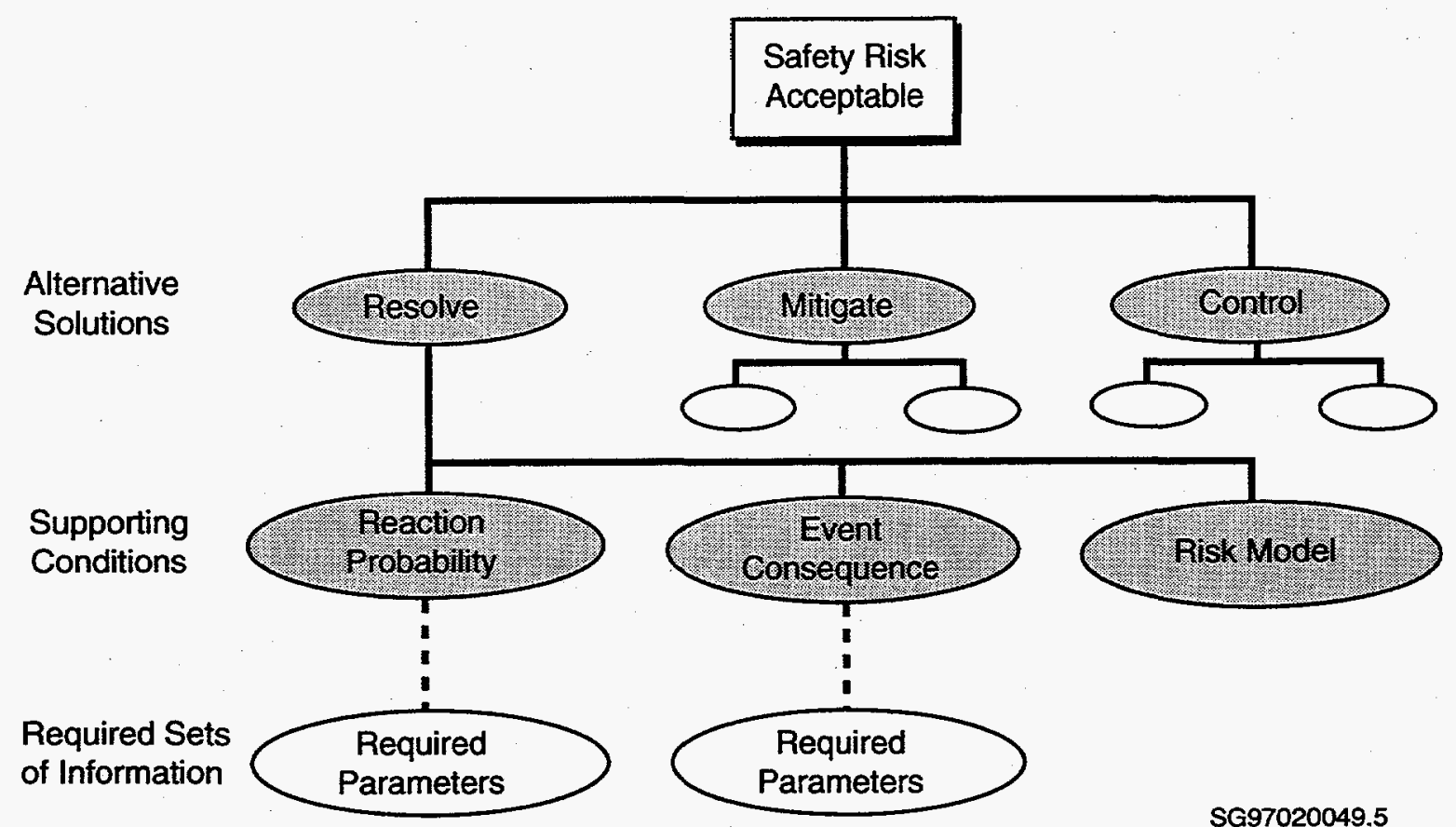

Figure 2.4. Underlying Thought Process for Preparing and Organizing Structured Logic Diagrams 
Creation of the structured logic diagrams requires qualified, multidisciplined scientific and technical Resolution Teams, whose capability base spans the chemistry, physics, and technology that the problem embodies. For the most challenging and enduring problems (e.g., safety problems caused by production of flammable gases by reactions within the tank waste), these teams become standing scientific panels that create and manage the development of the structured logic diagrams and overall implementation of the recommended strategy until the related objective has been accomplished. The technical capabilities of Resolution Teams and any associated consultants and staff are key to ensuring the successful implementation of the strategy.

As shown in Figure 2.4, the structured logic diagrams for safety issues are organized to consider the three primary options for issue closure: 1) resolve through knowledge that existing risks are acceptable, 2) mitigate to change the waste conditions sufficiently to make the risks acceptable, and 3) control to properly manage any tanks for which risks are known to be unacceptable. This approach is a disciplined means of developing an understanding of the physics and chemistry of the waste, its associated phenomenology, and how this phenomenology relates to risk. Furthermore, when the structured logic diagram is organized as in Figure 2.4, one works down the left-hand column and then to the right, recognizing that the normally preferred solutions are found in that order. For example, determining that the waste properties are such that the probability of a proposed event is incredibly small is the most desired solution. It provides the highest assurance that acceptable risk can be achieved without additional mitigation or control actions. Generally, mitigation is better than procedural or instrumental controls because it relies more on adjusting the waste's chemical or physical conditions to avoid an unwanted event than on workers adhering to work procedures. Likewise, mitigation is preferable to exercising controls. An exception would be the addition of water to a leaky tank. Obviously, in that circumstance, it would be better to implement a control to keep the risk acceptable. Using the approach described, the structured logic diagrams will be developed only to the extent that they enable defensible decisions.

This leads to a hierarchy when considering alternative solutions. For example, the resolution option can be achieved if the chemical reaction of concern is not credible, the frequency of the event is extremely low, or the resulting event is inconsequential. While in principle each has equal weight in the overall health or environmental risk calculation, a contained fire inside a tank, for example, could still have significant programmatic consequences. Thus, waste conditions in which reaction probabilities are very low would be the preferred outcome. A structured logic diagram developed along these lines would naturally reflect the degree of rigor needed to obtain the most valuable solutions. Although it would not reveal all possible solutions, the top-down, left-right development approach is designed to discover the most desired solutions first. Simple issues with relatively uncomplicated phenomenology will entail straightforward structured logic diagrams that can be quickly developed. For more complex, less straightforward issues requiring a rigorous technical analysis, the structured logic diagram will offer multiple paths to success and will provide guidance for evaluating how individual parameters are related to the overall risk. This leads naturally to the use of risk in decision making. Whereas the structured logic diagram is expected to be broadly applicable to tank waste remediation and disposal, logic diagrams for activities beyond safe interim storage are not identified or addressed in this document. 
In every case, the top event chosen for each structured logic diagram is the establishment of an acceptable level of risk for a specific safety issue. All basic components of a potential unwanted event (initiating factors, conditioning event scenarios, primary event scenarios, secondary event scenarios, release scenarios, possible source-term configurations and amounts, pathway scenarios, and waste uptake and consequence scenarios) are included in the structured logic diagram. The structured logic diagram shows the technical basis for four fundamental models listed here; each is an essential component of the tank safety analysis resolution strategy:

- A model of the phenomenology itself. A fully developed structured logic diagram includes the physical and chemical conditions related to a proposed action or decision, the frequency of potential reaction initiators, physical and engineering factors such as weaknesses or strengths of the containment vessel or its components, and the consequences of possible disruptive events related to the waste, including transport and fate models and long-term and secondary consequence possibilities.

- A model for uncertainty analysis that reflects the flow of information and propagation of uncertainty from tank conditions to the uncertainty in the estimate of risk.

- A model for evaluating the logical completeness of the tank safety analysis, including the systematic inclusion and analysis of new safety concerns.

- A model for systematic resolution of the large-scale composite tank safety problem that focuses on key data and information needs. By treating the structured logic diagram as a large-scale, decomposable problem, it is possible to eliminate, simplify, or combine subproblems for faster, easier, and more economical solutions.

Structured logic is a tool for visually presenting and analyzing the underlying, causative phenomenology for each safety issue that allows for systematic application of logic for problem resolution. The following sections discuss structured logic as a tool; how the structured logic diagram is used; how uncertainty analysis is applied to tank safety issues; how the structured logic diagram is used as a computational model for uncertainty analysis; and how safety issues raised by new hypotheses are identified and resolved.

\section{Structured Logic as a Tool}

A major issue in ensuring tank waste storage reliability is establishing standard methods to ensure a review of the technical basis that provides a systematic examination of all conceivable pathways by which a disruptive event can contribute to risk. Herein lies a potential pitfall. "Conceivable" means the ability of a human to conceive an idea, and this is a highly unpredictable variable. Structured logic is a standard tool in the chemical and nuclear industries, where it serves to optimize yields of highly characterized processes and reduce the risk of failures. Detailed descriptions of structured logic for each safety issue developed using this strategy and its depiction in the structured logic diagram are given in separate, unpublished reports (Doherty et al. 1996; Goheen et al. 1996; Liebetrau et al. 1996; Pulsipher et al. 1996; Stewart et al. 1996). 
Structured logic diagrams illustrate how failure can be eliminated and allow for a systematic examination of all identified ways that failure can be eliminated. By so doing, the most desirable approach(es) to eliminating failure can be identified from among all options.

There is danger in "blind" reliance on structured logic or any other decision support tool (Colson et al. 1996). The human element and lack of complete knowledge about the systems (e.g., tank waste) being evaluated naturally introduce unquantified uncertainty and the potential for errors of judgment. Many human-caused disasters have taken place in spite of very rigorous planning. Operations as complex as tank waste cleanup will not be at zero risk, even if structured logic and other quantitative risk assessment methods predict an acceptable risk margin for a given action. Because of their quantitative flavor, such methods may nurture a false security. Therefore, it is critical to ensure that the structured logic process is carried out in an open, disciplined, and scientifically defensible manner to lessen the chance for a major unwanted event to occur.

\section{Using the Structured Logic Diagram}

Addressing any one of the tank waste safety issues can require solving a few or many (more than 100 in some cases) smaller component problems of varying degrees of difficulty. These subproblems can range from data sampling problems to developing and applying models of the events that represent safety issues to be resolved, mitigated, or controlled. The speculative nature of much of the waste, the deteriorating condition of the tanks, and the difficulty of taking and analyzing samples of radioactive material make uncertainty a significant factor in evaluating tank safety issues. The uncertainty component of risk can be quantified in the structured logic approach and documented for each key parameter on the structured logic diagram. The uncertainty about noncritical parameters, properties, or chemical reactions, those with little effect upon risk, can be ignored. Acceptance of non-zero risk decisions requires that areas of uncertainty be acknowledged and understood and that their effect on potential actions be assessed.

Many of the identified component problems may be eliminated by identifying controlling relationships among them. These relationships will be identified in the structured logic diagram. For example, information about the nature and amount of fuel (e.g., organic waste) can dominate information about possible reaction initiators (e.g., spark or high heat source). If there is no fuel, reaction initiators are not a concern and the problem is resolved without a formal risk calculation. This leads naturally to a hierarchy of importance in knowing particular factors that contribute to risk. If the potential for an event to occur is sufficiently low, there is no need to know the amount of energy released, the effect of that energy on the tank, the amount and means of release of tank contents, how the contents are dispersed to the workers and the public, and the health consequences of the resultant exposure, if any. On the contrary, if the potential event is likely, then each of these additional data needs becomes important in a progressive manner depending upon the projected severity of the purported event. Hence, the strategy calls for the iterative development and application of the structured logic analysis of the problem. The selection of acceptable risk criteria also plays an important role in constraining the complexity of the problem analysis. The expectations of the public are that the frequency of events of the type that might represent a public risk (i.e., a small in-tank fire) must be maintained sufficiently low that the potential of a larger event with 
significant health and environmental risks becomes incredibly low. Managing to this more conservative measure of risk greatly simplifies the range of phenomena that must be contained in the problem analysis. It is important to establish risk criteria that are technically achievable and affordable:

\section{Uncertainty and Sensitivity Analysis Applied to Tank Safety Issues}

The logic and phenomenology captured in the structured logic diagram for each tank safety issue can be evaluated to estimate the expected value of risk for comparison with acceptability criteria, especially applicable regulatory or tank operation safety limits. However, for the comparison to be meaningful, the risk estimate must be accompanied by a reliable estimate of its uncertainty. At a minimum, the uncertainty should capture variability in model parameters, especially in the input data and any lack of knowledge about the phenomenological processes involved.

Quantitative risk uncertainty analysis is required for any process undertaken to provide a technically defensible estimate of risk. The analysis is incomplete and, in the worst case, can be misleading until such an estimate is determined. This is true whether or not the analyses rely on back-of-the-envelope calculations or complex numerical models.

Structured logic diagrams greatly facilitate the analysis of risk uncertainty by exposing the sources of uncertainty associated with each phenomenology and by helping to focus on the controlling drivers (parameters and reactions) in the analysis. The uncertainty model involves obtaining the known (or an estimate of) uncertainty for each data need and then, at each junction in the structured logic diagram, showing how variability in the inputs is propagated to the output(s).

Distributions that describe uncertainty in data needs or models can be propagated through a computational structure based on the structured logic diagram, which also serves as a convenient calculational template for uncertainty analysis. Uncertainty distributions can be obtained empirically through actual knowledge about error in the data collection processes or, theoretically, through numerical methods. Propagation of uncertainty through each of the alternative paths through the structured logic allows the nature and amount of uncertainty to be realized for each option under consideration in the decision and subsequent action recommendations. Uncertainty propagation is shown through a structured logic diagram in Liebetrau et al. (1996).

Uncertainty issues are at the core of each safety issue, and developing the uncertainty estimation strategy is crucial to the satisfactory resolution of every tank safety issue. Using structured logic, the decision maker is fully aware of the risk uncertainty in each potential decision. 
Using the structured logic diagram also assists in the sensitivity analysis of the data. Sensitivity analysis estimates the effect on a calculated output (i.e., level of risk) due to variation in one or more input parameters. A sensitivity analysis is undertaken for two reasons. First, to achieve a minimal representation of the model (in this case, the structured logic diagram and its key component models) by identifying noninfluential variables and components that can be ignored in subsequent uncertainty analysis. Thus, sensitivity analysis is a quantitative tool used to pare down the structured logic diagram to a minimal set of pathways essential to solve the safety problem. Because the analytical steps involved in sensitivity analysis are closely related to those for uncertainty analysis, the second reason to do a sensitivity analysis is to provide the basic information required for uncertainty analysis.

Uncertainty in risk estimates can arise from a variety of sources. One of the most familiar is measurement variability mainly due to sampling errors and, to a lesser extent, due to instrument errors. Uncertainty can also arise for other reasons such as a lack of information. The concentration estimates from the Historical Tank Contents Estimation model (Brevick et al. 1995a, 1995b, 1995c, 1995d; Agnew et al. 1996), for example, are uncertain because (among other reasons) the records used to derive them are incomplete. Uncertainty also appears in the form of incomplete or inadequate phenomenological models; that is, models that do not adequately predict the phenomenon they are designed to model. The structured logic diagram helps deal with the inescapable sources of uncertainty and variability in characterization by displaying how that uncertainty will affect or influence answers and where significant sources of variability lie.

Just how uncertainty is represented is important in any structure with the logical rigor of the structured logic diagram. There are many ways to represent uncertainty. These include the variance, the range determined by the highest and lowest possible values, and tolerance intervals. The usefulness of these measures depends on how they are derived and what form they take. The variance, for example, is not appropriate for characterizing the variability of a parameter that has a highly skewed or bimodal distribution. The one representation that always contains all the relevant uncertainty information for a given quantity is its distribution function. In fact, all other representations of uncertainty can be derived from the distribution function. Empirical distribution functions derived from observational or experimental data measure analysis variability. A distribution function may also be selected for theoretical reasons. In all cases, the distribution functions are formally treated alike in the subsequent uncertainty analysis. The importance of uncertainty analysis in safety issue resolution is further discussed by Liebetrau et al. (1996).

\section{Identification and Resolution of Safety Issues Raised by New Hypotheses}

Confidence in the structured logic is based on an accurate data and problem description plus rigorous expert analysis. Such an approach can give the false impression that complex, dynamic systems can be definitively understood. Therefore, a process to account for the discovery of new sources of risk is described further in Colson et al. (1996).

By their nature and history, Hanford tank wastes preclude the a priori definition of all possible safety concerns. In some cases, it is uncertain what material and material quantities have been placed in the tanks. Furthermore, the wastes are chemical reaction systems that continually produce new substances as 
the organic complexants and solvents undergo radiolysis and chemical conversion. Hence, while it is important to encourage the ongoing generation of new hypotheses about and analysis of potential safety issues, it is also important that the new hypotheses pass a credibility test before they are allowed to affect tank waste characterization and management decisions. A formal and open process will assure all concerned parties that the identification and analysis of potential safety concerns is being given appropriate attention. The recommended strategy provides a logical approach for resolving questions raised by a safety-related hypotheses. It is important to note that, since 1990, only six major safety issues related to the tank waste itself have been identified, and several have been resolved or are approaching resolution.

An example of the strategic handling of questions raised by new hypotheses has been developed in Colson et al. (1996) and illustrates the

- dynamic and sometimes unknown chemical nature of the waste

- potential consequences of prolonged storage of the waste

- importance of validated chemical models in studying the waste and asking speculative "what-if" questions

- ability of the recommended strategy to address new issues.

New safety concerns may arise as additional information becomes available about the waste and/or as its properties are modified through waste retrieval and management. The first response is to develop a technical analysis of the potential existence of a problem (i.e., are the proposed circumstances credible and, if so, do they represent a significant risk?). Using information in the scientific literature about the proposed phenomena and using existing data about the tank contents, a model is created to test the safety risk hypothesis. The validity and quality of these data are being considered. New issues can bring new data quality and completeness requirements. For example, a more complete analysis of existing mass spectrum or optical spectral data may be required to look for species previously not thought to be important. Evaluating many of the suggested concerns will be accelerated because they naturally fit within the structure of existing structured logic diagrams, appearing as new energetic species or reaction initiators. For concerns that pass the initial credibility test, the strategy is then fully exercised as information needs are justified and met, and the findings are used to decide on appropriate actions. This type of problem emphasizes the value of an iterative strategy in which screening information (e.g., looking for species that are indicators of the existence or absence of the proposed safety conditions) is obtained before a more costly sampling and analysis campaign is undertaken to prove or refute the validity of the proposed safety concern. 


\subsubsection{Justify Information Needs}

Sampling and analysis are costly and time-consuming and involve risky actions. Therefore, the recommended strategy does not assume a waste sampling and analysis baseline for obtaining required information. Only information that reduces waste storage and disposal risks to acceptable levels is pursued.

To provide a technically defensible and economically justifiable basis for waste characterization, the recommended strategy employs an approach that explicitly links sampling, waste analysis, physicalchemical modeling, and other learning activities to risk reduction and decision making. After the technical basis for decisions has been developed and reviewed, the knowledge that is genuinely needed to make better decisions must be determined. Justification for additional information requires specific evidence that it can reduce uncertainty in health and environmental risks or potentially change a decision about the system. If new data or the information derived from those data cannot reduce uncertainty, they are not justified and should not be collected. If new data will not change a decision based on current information, they are not justified and should not be collected. If new data are not justified, the next appropriate steps are immediate decision making for this issue. In Figure 2.2, this is shown by the direct line from Section 2.3.3 to Section 2.3.8 (from Justify Information Needs to Make Decisions).

A tool that can be used to analyze risk-based requirements in complex problems is value-ofinformation (VOI) decision analysis (Raiffa 1968; Keeney and Raiffa 1976; von Winterfeldt and Edwards 1986; Clemen 1991). Simply stated, the output of this analysis indicates that if the cost of acquiring additional information is greater than the expected costs of a wrong decision made without the information, then the information is not worth obtaining. The result is a clear measure of completion. Characterization for a given action or decision is complete when the costs of additional characterization activities exceed the calculated value of the information for decision making or risk reduction. Conversely, additional waste characterization is justified when the calculated value of new information exceeds the cost of obtaining it. This decision analysis approach is to be applied with a degree of rigor that is tailored to the level of difficulty and complexity of the issue being addressed. While not burdening straightforward problem analysis, it enables effective decision analysis of complex problems.

In addition to guiding characterization decisions, knowing the value of obtaining specific information will provide an explicit basis for investments in research and/or technology development to reduce health risks, costs, and technical uncertainty.

The technical analysis of each desired outcome (see Section 2.3.2) results in a set of alternatives ("paths to success") and associated information needs. The challenge is to select an acceptable alternative that achieves the desired level of risk at the lowest cost. The attributes of the selected alternative then provide the fiscal and technical justification for needed information. 


\section{Risk and Fiscal Justification of Characterization Work}

A preliminary risk evaluation is conducted using the best available information, including its uncertainty, as described in Section 2.3.2. This evaluation serves two purposes. First, it provides an initial look at where an issue or decision lies with regard to a desired risk value. To illustrate, Figure 2.5 shows four hypothetical risk evaluations for four tanks, with associated risk uncertainties given in the vertical lines. For example, the uncertainty associated with the risk estimated for Tank 3 is much greater than for Tank 2. The acceptable risk criterion is given as the horizontal line. For many industrial practices, this criterion represents a one-in-a-million chance that an unacceptable event will take place. If the predicted risk, including its uncertainty, is significantly below or above the acceptable risk threshold (as in Tank 1 and Tank 2), the issue will be summarily resolved (Tank 2) or will require mitigative action (Tank 1). There is no role for additional characterization in either case if, in fact, the problem has been properly described and analyzed. Because the uncertainty in the risk of Tank 3 crosses into the acceptable risk criterion value, additional characterization may be beneficial to determine whether the safety issue in this tank must be mitigated or controlled. Decision makers must make that judgment. On the other hand, the risk of Tank 4 is mostly in the acceptable range; however, there is enough uncertainty in the risk estimate that the chance of an unacceptable risk existing merits more assurance that its risk is indeed acceptable. Thus, there is a strong basis for collecting additional characterization data. Sensitivity analysis (see Section 2.3.2) can then be used to identify the solution paths that have the highest likelihood of success and the data needs that have the greatest effect on the overall risk calculation. As a result, further analysis of the value of information is less complex and focuses on the highest payback items. The inherent structure of the decision model allows for quantitative probabilistic estimates of the risk associated with the decision alternatives to be generated. Once this is done, comparison with established risk acceptance levels and evaluation of cost/risk-reduction tradeoffs become possible.

The biggest challenge in using this approach is in obtaining a high degree of confidence in the estimate of uncertainty in the calculated risk. Hence, when making decisions with significant consequences, it is important to evaluate carefully the validity/uncertainty of data that has the largest effect on risk uncertainty. Value of information is defined as the difference in the expected value of making the decision based on existing knowledge and making the decision with better information. Existing information consists of prior sampling and analysis data, results of tank content models (e.g., Agnew et al. 1996; Brevick et al. 1995a, 1995b, 1995c, 1995d), and/or expert opinion. Value-of-information decision analysis places a distinct burden-of-proof on those who want better information to show how it will benefit decision making. A decision to undertake additional characterization work must compete for resources with decisions to undertake other activities such as mitigative or treatment actions or to accept the risk of not taking any action. 


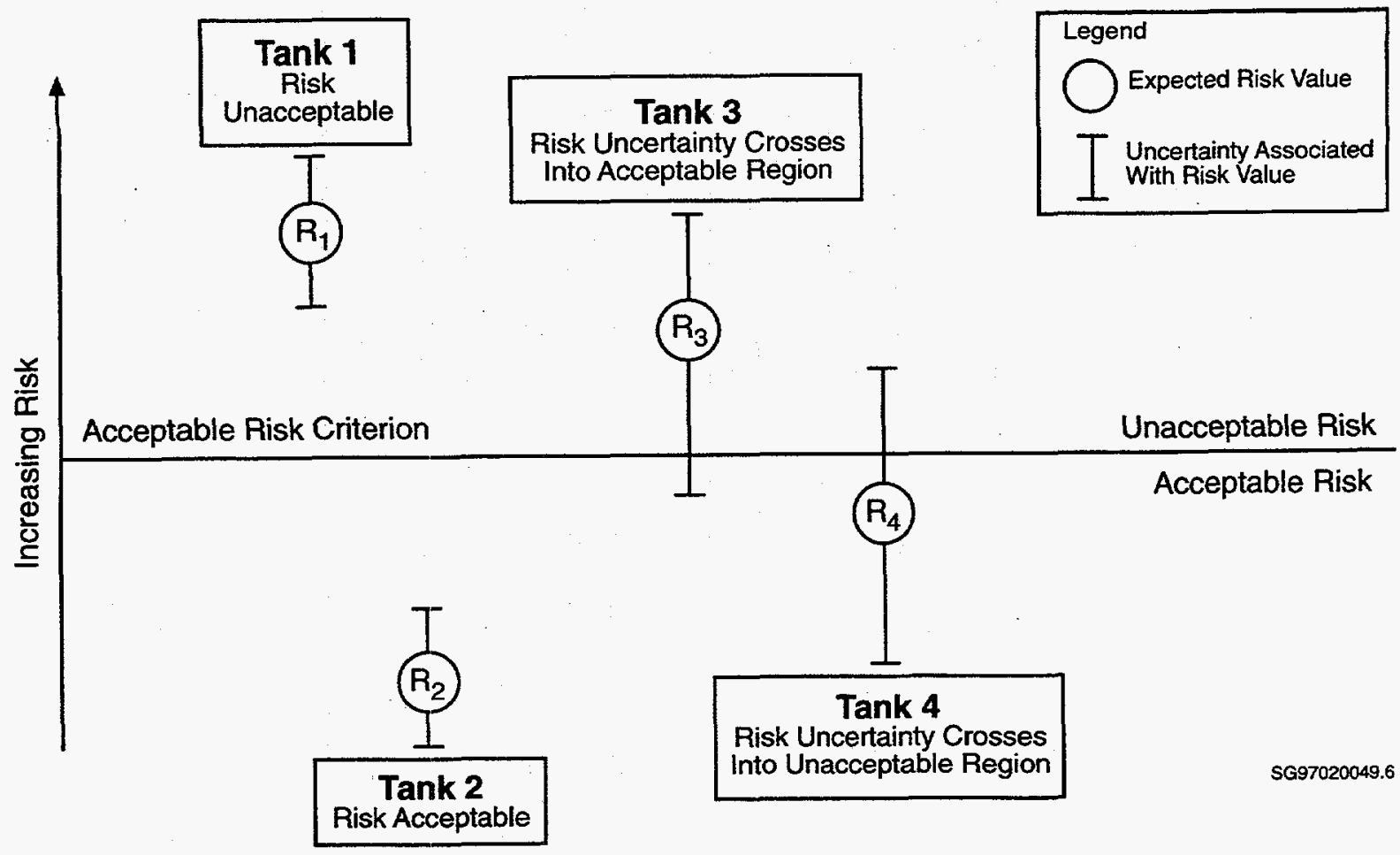

Figure 2.5. Example of Preliminary Risk Evaluation

The technique initially calculates the value of perfect information (i.e., information that would allow a decision to be made with no uncertainty about the input data). Because all real data collection activities will entail some uncertainty, the value of perfect information represents the upper bound of how much it is worth to know a specific piece of information, i.e., a limit to the resources that should be allocated to obtain that information. Many potential characterization options can be ruled out because the characterization cost is greater than the value of perfect information.

The value of information decreases as the quality of the information decreases. An example for the organic-nitrate safety issue is shown in Figure 2.6 and is described in Section 4.1. For this example, information about tank contents is described by the amount (volume) of reactive waste within increasingly larger increments of tank waste. Reactive waste is measured by the combined fuel and moisture content that can cause a condensed-phase organic-nitrate reaction. This figure presents the expected value of information as a function of information quality for four actual Hanford tanks: S-105, TX-102, U-109, and U-107 (see Colson et al. [1996] for locations of tanks). One measure of quality is the relative standard deviation (RSD), which is the standard deviation divided by the mean. Perfect information has an RSD of 0 . The quality of information decreases as the RSD increases. The $y$-axis in Figure 2.6 presents the expected value of information (for knowing the amount of reactive organic waste) in thousands of dollars, and the $\mathrm{x}$-axis shows the information quality. Although a general trend of decreasing value as a 


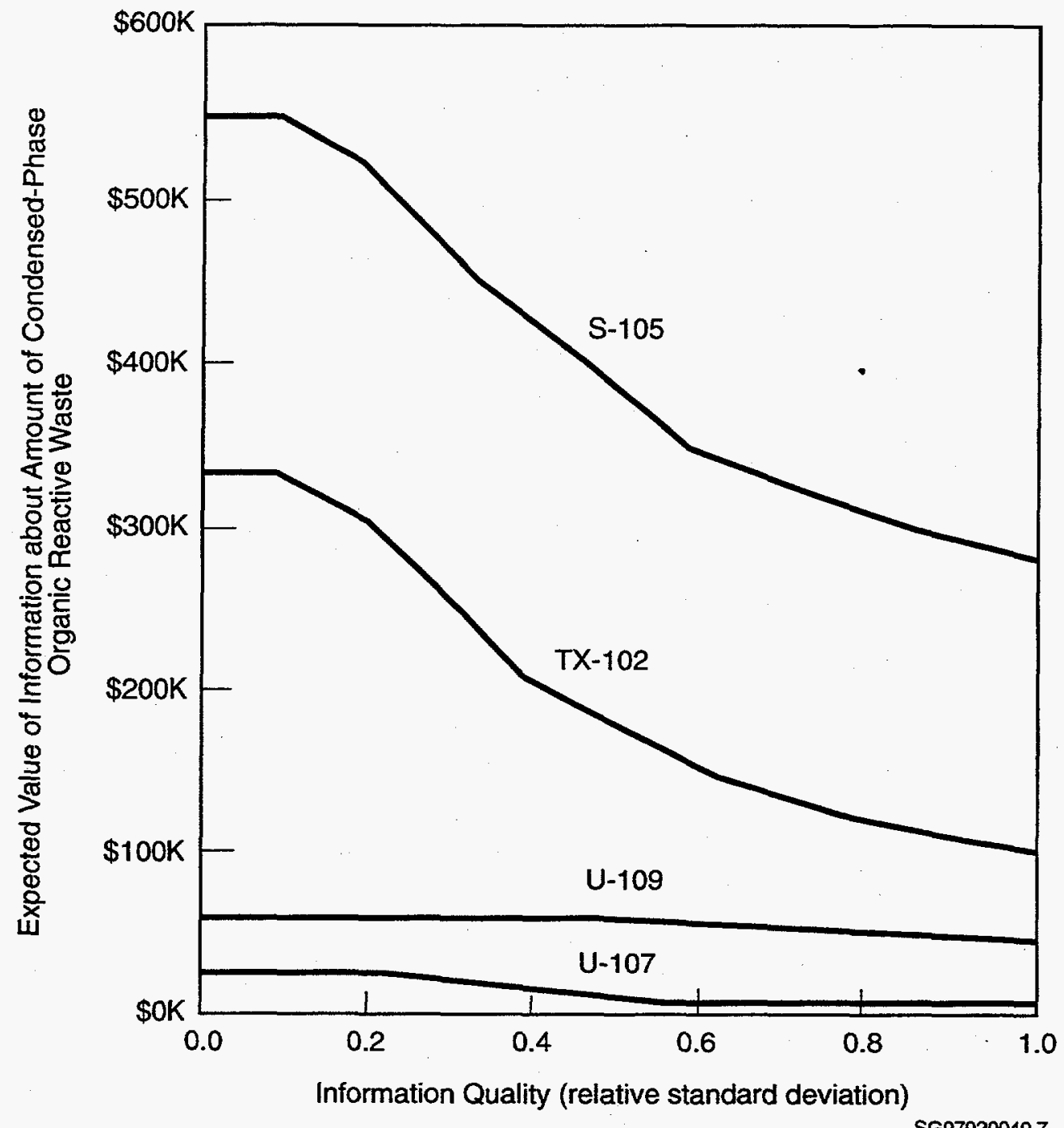

Figure 2.6. Example of Value of Information as a Function of Uncertainty

function of decreasing quality can be observed, the slope and shape of the loss curve are very much tankdependent. For example, these curves are affected by the availability and quality of prior information, the proximity of the prior estimate to the decision threshold, and the cost effectiveness of potential mitigative actions.

Additional data from Tanks U-109 and U-107 generally have a low value of information. Controlling ignition sources is an effective and inexpensive alternative to reducing the risk from these wastes. The availability of an effective, low-cost option limits the value of collecting additional information. These two tanks are also relatively insensitive to information quality because the information has very little effect on the decision under consideration. If no changes in decisions are affected by changes in information quality, the value of information will remain relatively flat, such as for Tanks U-109 and U-107. 
Tanks S-105 and TX-102 show a different scenario. The prior information cannot establish whether the waste is sufficiently damp to be unreactive. The recommended action (do nothing or conduct mitigative actions) is highly dependent on whether the waste is reactive or damp. Information on these parameters can greatly affect the decision and, therefore, establish a value for that information. For these tanks, the recommended action changes as the quality of information changes. Therefore, there is a relatively sharp decrease in the value of information as a function of decreasing quality.

The conclusions that can be drawn from the curves in Figure 2.6 are following: If resolving the organic-nitrate safety issue was the only concern associated with those tanks, further characterization of Tanks U-109 and U-107 would not be justified. However, gathering information from Tanks S-105 and TX-102 may be justified if it can be collected at a given level of quality for less cost than the value of information at the same level of accuracy. For example, if this information could be obtained at a quality level of 0.6 relative standard deviation (RSD) for a cost of $\$ 300 \mathrm{~K}$, then further characterization would be justified for Tank S-105 but not for Tank TX-102. These curves provide a finite "completion" criteria. The implementation of this value of information requires integration across all needs for information (see Section 2.3.5).

\section{Technical Justification of Characterization Work}

Technical justification of characterization work relates to the need for new information for decision making and to the feasibility of acquiring information. The justification must be rigorously and consistently applied in a scientifically defensible manner. It must be determined whether it is technically feasible for the planned approach to provide information of sufficient quality to effect decisions. Otherwise, costly and time-consuming waste characterization campaigns have no technical merit. Technical justification is a critical factor in gaining cost control and defensibility for waste characterization activities.

Once the required data quality is obtained, sampling ceases. For some issues, resolution may not require information about the average tank content, thus greatly reducing waste sampling constraints. For example, resolution of the ferrocyanide safety issue required only the knowledge of a ratio of constituents (nickel and cyanide) to be determined in tanks that had received ferrocyanide- and nickel sulfate-bearing wastes.

\subsubsection{Use Waste Actions as Characterization Opportunities}

The principal technical difficulty in the successful characterization of waste and the resolution of safety issues originates in the chemical diversity of the waste types and their physical, chemical, and radiological heterogeneity. Attempts to "completely characterize" sludges, slurries, saltcakes, crusts, and other forms of solid-laden materials are so severely restricted by the requirement for many randomly selected samples that the approach is impractical. The recommended strategy circumvents this problem by developing and taking advantage of a wide range of waste actions as opportunities for waste characterization. For example, obtaining data during waste actions reduces the risk and uncertainty of future decisions because it provides understanding of the behavior of tank waste during relatively large-scale perturbations. Actions on waste (waste operations and processing functions) are seen as key opportunities to obtain 
characterization information. Complex, inhomogeneous systems such as the Hanford tank wastes are extremely difficult to "characterize" using a waste sampling approach in their current stored state. However, when the waste is disturbed, measuring the response of the waste system is a standard approach to characterization that is often used in industry. Examples of such characterization opportunities may include obtaining grab samples or on-line measurements in waste transfer lines and conducting vapor, liquid, and solid sampling during and after actions to retrieve waste. At these times, better characterization information can be obtained using fewer operational and financial resources than required to obtain representative sampling and analysis results from a chemically and physically complex waste system. For example, salt-well pumping has been shown to release gases that were trapped within the waste (WHC 1996). Simultaneous sampling and analysis of these gases will provide information on 1) their amount and flammability for understanding the flammable gas safety problem, 2) the existence of any new safety concerns (see the discussion on safety issues raised by new hypotheses in Section 2.3.2 and Colson et al. 1996, Appendix B, Section 3.5), and 3) decomposition products from organic precursors that are important elements in organic waste aging models (Webb et al. 1995).

Applying the concept of the transfer function (the ratio of the system input to the system output, where the system input is perturbed and the response is measured), the response of the tank waste system is predicted and then measured. The response of any measured parameter compared with its predicted value can validate the model or identify where improvements are required. For example, resolution of the flammable gas safety issue requires a model of the amount and composition of stored gas in each tank or group of tanks. Actions that remove liquids from a tank predictably reduce the gas storage capacity of the remaining waste. Monitoring changes in the type and concentration of head space gases will test the gas retention model for that tank. Major actions such as salt-well pumping and tank-to-tank waste transfer are justified opportunities to obtain valuable characterization information.

Acquiring waste data when the waste is being disturbed will add costs and complexity to waste management and processing work and therefore also must be justified by analyzing the value of information expected and comparing it with the additional costs. The proper application of such an "opportunistic approach" significantly increases the effectiveness of waste characterization work by using waste operations and processing functions (including work by privatization contractors) as a cost-effective method to collect additional information from large waste volumes. The approach requires modification of operational practices to allow for the addition of characterization instruments and work. These opportunities must be carefully designed to provide the highest return on investment in waste characterization. The recommended strategy also acknowledges that characterization is not a one-time event. As wastes proceed through the interim storage/treatment/disposal life cycle, their composition and characteristics will be altered either naturally or through operations actions.

The contractual framework in which privatization vendors and support waste management functions do their work needs to support characterization as a risk management tool. For example, the preparation of waste feeds for use by vendors should support characterization needs broader than just performing vitrification demonstrations on that feed. It is also recommended that the privatization contracts require the contractor(s) to disclose extensive data about the waste composition, properties, and behavior while 
being treated-data that they might otherwise consider proprietary. Especially during the early years, these data will provide valuable information about the waste that remains in the tanks from which the waste was retrieved for delivery to the privatization contractor and about waste in similar tanks.

\subsubsection{Integrate Requirements and Plan Characterization}

The output from the risk-based and value-of-information requirements analysis process provides an explicit and potentially quantitative basis for integrating characterization needs from each issue and each tank to form a complete, comprehensive, and defensible characterization plan. This integration leads to the appropriate selection of sampling and analysis, monitoring, special studies, and laboratory experiments to form an implementation plan for a defensible and cost-effective characterization supporting the waste disposal program. This approach will also provide a defensible basis for budgeting and scheduling decisions by justifying and prioritizing characterization work for funding. Characterization work conducted in this context will become a cost-saving investment because it selects only those characterization actions that are less costly (more valued) than taking alternative actions to achieve waste safety or disposal goals without the additional information that additional characterization work would provide.

Characterization decisions will not be based solely on individual issues. The risk-based requirements analysis process estimates the value of information about a specific issue in a given tank. However, characterization decisions will be based on the combined need for information in a given tank or for the combined value of knowledge gained from quantifying a particular parameter or chemical process across multiple tanks versus the cost of obtaining it. Techniques for combining value of information across issues and tanks are addressed in Section 4.

Decisions regarding further justified characterization and the relative tank priorities discussed above provide a defensible basis for preparing short- and long-term characterization plans and optimizing characterization schedules. Individual tank characterization plans will be developed for each tank, group of tanks, or waste actions based on the combined value of performing characterization activities in that tank. These plans document the rationale for collecting or not collecting further data.

\subsubsection{Collect Data}

Data collection activities will be conducted based on the tank waste and waste actions characterization plans. These activities can include historical records retrieval, laboratory experiments (e.g., aging of organic wastes), on-line monitoring of tank conditions (e.g., hydrogen monitoring sampling and analyses, results from modeling [e.g., tank layering model]), or review of work in the scientific literature.

Raw data are collected in any of the methods listed above, then processed and validated to become useable information for the Resolution Team. It is critical that these data accurately represent the parameters the Resolution Team requires for making technical decisions. 


\section{Historical and Expert Judgment Data}

One source of information is historical data available on a tank. If the source is sufficiently reliable, then the information would be provided and the request satisfied. An example of a historical data source is the Historical Tank Contents Estimation model (Brevick et al. 1995a, 1995b, 1995c, 1995d; Agnew et al. 1996). This information is based on the use of historical processing and waste transfer/storage records to infer current tank contents. The most reliable historical data sources will have been validated and peerreviewed to ensure the required data quality. These data sources have not been validated by external experts. Much of the historical raw data must be combined with numerical modeling (e.g., radiological aging of components and physical layering) to produce valuable information.

Another method for developing estimates of parameters is to elicit the values based on expert judgment. This method is an adaptation of the Nuclear Regulatory Commission (NRC) methodology documented in the NUREG-1150 risk analyses of several nuclear power plants (NRC 1989; Keeney and von Winterfeldt 1991). The NUREG-1150 methodology involves a formal elicitation of probabilities using expert judgment combined with extensive existing data and model calculations. Some of the important features of the methodology are

- clear definitions of the parameters and variables to reduce ambiguities about what is to be elicited

- careful selection of experts to preserve a broad range of approaches and diversity of opinions about the variables

- training of the experts in expressing their judgments as probabilities and probability distributions

- aggregation of the expert judgments to preserve the range of opinions and approaches

- documentation of the results in a form that allows reviewers to scrutinize the reasoning of each individual expert.

Recent studies of flammable gas safety have relied on this method for estimation of some parameters and are summarized in Section 4 . Elicitation of values from experts should only be used when sufficiently reliable data are not otherwise available. Details on the elicitation process are provided as well.

\section{Experimental and Modeling Data}

Experimental data are another source of information. Laboratory experiments are performed on actual (or simulated) waste to provide information about phenomena and to estimate key parameters. For example, important information has been provided in this manner about the mechanisms of the formation of flammable gases, the decay of energetic compounds, and the distribution of chemical species among different phases within the waste materials.

Modeling data result from mathematical representations of physical and chemical phenomena. Model- 
Modeling data result from mathematical representations of physical and chemical phenomena. Modeling results are used when a first estimate of a parameter is required, or when it is difficult to measure an actual value because of time, costs, or operational constraints. Existing tank layering models, organic aging models, and tank waste grouping models are examples of numerical representations of actual phenomena. Models also help to interrelate divergent data sources. For example, it may be possible for modeling (e.g., a chemical reaction model) to relate information about the liquid and vapor phases (information retrieved via sampling or monitoring) to the contents of the solids in the same tank.

\section{Monitoring, Sampling, and Analysis Data}

Tank monitoring data are parameters such as in situ temperature, liquid level, and vapor space measurements. Acquisition of these data has some of the same vulnerabilities as retrieved samples (e.g., grab, auger, or core samples) in terms of how well the data represent actual tank conditions.

One of the most vulnerable points in any characterization strategy is the acquisition of samples. This applies both to physically removing the waste for subsequent ex-situ analysis or to placing an in-situ measuring device in the waste. Sampling of the nearly homogeneous liquid and vapor phases in the tanks avoids the major concerns about sample representativeness that strongly affect the value of sampling thick slurries, sludges, and saltcakes (see Colson et al. 1996, Appendix B, Section 2.3.1). The solid phases of the wastes are typically heterogeneous and not necessarily in equilibrium with each other or with the liquid and vapor phases. A defensible strategy for justification of solids sampling requires consideration of the number of samples, their location(s), the waste handling, and processing requirements for analysis. Any sampling plan will be constrained by the locations of access. Existing access ports into the tanks 1) limit the use of random or fixed grid sampling schemes, and 2) may be over waste that is not representative of waste in the entire tank. A potential resolution of this problem is to use the technology needed to sample the tanks at any location (e.g., flexible robotics) or to characterize the wastes after they have been removed from the tanks. Another method is to use waste perturbations as characterization opportunities by predicting and measuring the system response to change (see Section 2.3.4).

Statistical modeling approaches (e.g., models of lateral heterogeneity, grouping tanks by waste types [Hill et al. 1995], etc.) may prove useful in specifying the number of samples required. Once the required data quality is obtained, sampling ceases. For some issues, resolution may not require information about the average tank content, thus greatly reducing waste sampling constraints. For example, resolution of the ferrocyanide safety problem required only the knowledge of a ratio of constituents (nickel and cyanide) to be determined in tanks that received ferrocyanide-bearing waste.

\subsubsection{Process, Manage, Validate, and Model Data}

A strong data management system is vital to the success of the recommended strategy. Data collected will be systematically captured and preserved by a reliable data management system. The data management system will provide the data and data quality information to the Resolution Team and all interested technical experts, decision makers, and stakeholders. Openness is key to maintaining the involvement of the broad scientific community in assuring the quality of the approach and the results. Electronic access to 
characterization information is also a requirement of the Tri-Party Agreement (Ecology 1996). The information structure provided by the structured logic diagrams will provide an important guide to organization of the information in the database.

Commonly, data by themselves do not constitute information. Information is derived from the data through chemical, physical, and/or mathematical models that relate the data to the phenomena of interest. Data that have been obtained for a particular purpose may or may not prove to be useful in responding to other information needs. If data do not meet the quality standards for the intended purpose, other means must be derived to obtain the required information. If the results fundamentally change the understanding of the problem, the characterization strategy will iterate back to the technical basis development/refinement step to benefit from this new understanding.

It is also important to evaluate objectively the quality of information relied upon for decision making because technically and economically important decisions depend upon using information of known quality. The defensibility of subsequent action depends upon the technical and scientific soundness of the inputs and. the decision process followed. For example, in determining the moisture content in solids, precision is only important near a threshold value. The waste type varies sufficiently from sample to sample to affect how each measurement should be performed. Thus "data quality" can be damaged rather than enhanced by using a nationally certified and traceable method for generic solids. Data quality must also be evaluated by independent (free from conflict of interest) and technically qualified personnel.

\subsubsection{Make Decisions}

If the execution of the recommended strategy is sound, results will promote making well-documented, defensible decisions that lead to actions with acceptable risk. The objective of the entire strategy is to guide the application of characterization resources so that their use is technically defensible, economically justified in the context of acceptable risk, and advances the safe storage and successful disposal of Hanford tank wastes.

The inputs to safety issue resolution decisions are the appropriate risk constraint measures, understanding of the parameters driving risk, and the risk-based decision model(s) for the issue. If the current state of knowledge satisfies all of the appropriate risk constraint measures, then the safety issue is resolved and the desired outcome is achieved. Thus, a perceived problem will have been resolved through understanding that the properties of the waste pose no unacceptable risk due to the related safety concern, and no mitigation or control actions are required as long as the controlling properties of the waste remain within acceptable limits. If the issue cannot be resolved through understanding gained from existing information, then risk reduction tradeoffs will be evaluated. These include taking mitigative action, establishing operational controls, or performing a more thorough evaluation of risk and cost consequences. Mitigative actions can either permanently mitigate the hazard or conditionally mitigate the hazard so that continued intervention becomes necessary. Installation of the mixer pump in Tank SY-101 is an example of conditional mitigation. Operational controls do not remove the hazard, but attempt to reduce, for example, the occurrence of initiators that might trigger a disruptive event such as a flammable gas explosion. 
Disposal decisions also begin with the same set of inputs. The appropriate set of risk measures will be more far-reaching than those for safety issue resolution decisions and will encompass issues such as longterm environmental risk, sociocultural risk, and programmatic risk in addition to the human health risks that dominate decisions related to safety issue resolution. These risk constraints and the methodologies to develop meaningful risk constraint levels are detailed by Harper et al. (1996).

If a prospective decision alternative meets all established decision criteria (risk constraint measures), then action can be taken directly to achieve the desired outcome. However, if some criteria are not satisfied by the best alternative, then further interaction with regulators and other representatives who work with the DOE to establish the decision criteria may result in a renegotiated basis for action to achieve the desired outcome. If neither of these paths are attainable, further evaluation of the problem will be required. A successful implementation of the risk management strategy requires that the parties involved in the decision-making process plus technical staff have a mutual understanding of the quantitative aspects of risk and cost-benefit calculations (Section 2.3.3).

\subsubsection{Take Action}

The goal of the outcome-focused strategy is to take action towards achieving the desired waste safety and disposal outcomes under the conditions of well-managed risk. The types of actions being considered are 1) those designed specifically for obtaining waste characterization data, 2) mitigative actions such as salt-well pumping or adding mixers to tanks, 3) addition of monitors and/or controls leading to improved operational safety, and 4) actions on the waste in direct support of waste disposal (i.e., preparation and delivery of waste for processing). When fully developed, the recommended strategy will provide the basis for justifying such actions. It will provide decision makers with the projected risk reduction benefits and with information about the risks and inherent uncertainties involved in taking specific actions.

When sufficient knowledge has been accumulated, a decision will be made by the DOE or its contractor personnel from prospective alternatives. Generally, the most effective alternative in terms of risk reduction, cost-effectiveness, and public acceptance will be selected. If a solution is found in which the risk, cost, and schedule are all acceptable, the proposed action will be taken. If no such solution is available, the process iterates back to evaluation of the technical basis to develop new alternatives or solutions. Key criteria in the justification of characterization actions are that their cost should not exceed the value of the information gained for guiding decisions about other actions.

If a prospective decision alternative meets all established decision criteria, then action can be taken directly to achieve the desired outcome. Note that 1 ) the choice of an action is made by individuals and will not be without risk even when based upon rigorous logic and quantitative risk analysis, and 2) the decision to not take any action also carries a certain amount of risk and must be technically defensible.

The recommended strategy also incorporates characterization actions into other actions that significantly perturb the waste. Properly designed characterization work conducted while taking actions will provide further knowledge that will refine the technical understanding for resolution of other issues and future decisions. Such events represent unique opportunities to gain further knowledge about the wastes. 
Any action affecting large volumes of the waste can be seen as a deliberate perturbation of the model for that tank or group of tanks and should be used to refine the model. An example of applied characterization accompanying an action is evaluating the temporal fluctuations of the hydrogen concentration in the dome of Tank 241-SY-101 following pump installation. To take advantage of these opportunities, the planning and approval of characterization actions must be rapid and flexible. This, in turn, will require reassessment of the current operational constraints on obtaining access into and on conducting studies inside the tanks to take better advantage of these characterization opportunities. 



\subsection{Value of Information Methodology}

The goal of a characterization strategy is to provide timely information to support decisions. Information has value if it leads to better decisions, which are those with better expected consequences. Thus, the VOI should be gauged by the increase in the expected value of decisions. Frequently (but not always), information will reduce uncertainty about consequences. Information will also often (but not always) lead to decisions that involve reduced risks. These ideas are made specific in the VOI approach described in this report for developing a characterization strategy to support tank safety and disposal decisions.

\subsection{Basic Approach}

VOI is a central concept in decision analysis (Raiffa 1968; Keeney and Raiffa 1976; von Winterfeldt and Edwards 1986; Clemen 1991). Qualitatively speaking, a source of information (such as a sample in a tank) is valuable if it has the potential for changing subsequent decisions, such as whether or not to keep TRU waste separate from HLW. For a source of information to be valuable, two conditions must be met:

1. The decision alternatives must have uncertain consequences (otherwise there is nothing to be learned from the information).

2. Depending on which information is obtained, different decisions may be best (otherwise, the information would not have any impact).

To conduct a VOI analysis, one typically first conducts a decision analysis without explicit consideration of the information source. This analysis consists of describing each decision alternative in terms of several decision criteria (e.g., health and safety risks, cost, schedule). This creates a matrix of alternatives by criteria, with entries being the best engineering estimates of how well an alternative meets the criteria. In this process, key uncertainties and risks will be identified. These are then structured in a decision tree.

The generic steps in risk-based VOI analysis include the following:

1. Develop a list of issues that must be addressed and an understanding of them.

2. Develop a survey of decisions that must be made.

3. Identify the major issues in each decision (i.e., the uncertainties and trade-offs that make the decision problem complex).

4. Qualitatively assess the value of different types of tank characterization information to support these decisions. This assessment will identify which decisions will benefit most from additional tank information. A quantitative VOI analysis would be worthwhile for these decisions.

5. Select a specific decision that merits a quantitative VOI analysis. 
6. Generate a list of tank characterization information that is a key driver for the selected decision. This could be scientific understanding, historical information, or sampling data.

7. Make a qualitative assessment of whether collecting this information (i.e., tank waste characteristics) would improve the decision by raising the following three questions:

a. How strongly does the decision depend on the information? Classify the dependency of the decision on the information as either none, weak, or strong.

b. If the decision depends on the information, how much can be gained by knowing the information? Assess in approximate terms what the costs and benefits of each decision are, given different states of the information and different prior probabilities.

c. Does collecting this information require a special effort, or will this information be available with little or no effort prior to making the decision? If the information will be available at little or no cost prior to decision making, it is not worth spending any additional effort or expense now to collect it.

8. Identify sources of information (e.g., historical records, sample data, laboratory experiments, statistical models, expert judgements).

9. Formally assess prior probability distributions over the tank waste characteristics that were determined to be key decision-drivers and for which it was determined that collecting the information would improve the decision. Quantitative distributions would be preferable to elicitation if such distributions were available. If quantitative distributions are not available, this step should be done with a formal elicitation of expert probability judgments based on NUREG-1150 (NRC 1989). The sub-elements of this step are

a. Identify the Hanford experts that are most knowledgeable about prior distributions for the key tank waste variables identified in step 6.

b. Conduct a meeting with these experts to clearly define the variables, train the experts in probability assessment, and define the elicitation tasks.

c. Have the experts conduct reviews and analyses to summarize their current state of knowledge about the key tank waste variables.

d. Conduct a second meeting with the experts to elicit probability distributions over the variables.

e. Document the elicitation results.

f. Analyze and aggregate individual probability distributions. 
10. Describe each decision alternative in terms of decision criteria and create an alternatives-by-criteria matrix.

11. Identify all key risks and uncertainties.

12. Structure these risks and alternatives into a decision tree showing the sequence of actions (squares) and uncertain events (circles) that describe the decision setting, and the criteria used to characterize the consequences of the combinations of actions and events.

13. Assess costs for all paths through the decision tree.

14. Assess the probability of occurrence associated with each uncertain event in the decision tree. Use the prior probability distributions obtained in step 9 or do additional assessments if necessary.

15. "Roll back" the decision tree from right to left to determine the expected value of each event node by summing the products of probabilities and costs for all the node's branches. The expected value at a decision node is the lowest expected cost from among the node's branches, which corresponds to choosing the alternative with the lowest expected cost. Repeat the process until expected values are determined for all nodes. Processing the tree in this manner provides a complete specification of the best decisions at all decision nodes and a determination of the VOI. The expected VOI is the difference between the expected value of the best decision without gathering the information and the expected value of the decision to gather the information.

16. Iterate as necessary among the steps described above.

In this section, the basic VOI approach is illustrated with a complete, simplified example of a VOI model. The structure of a VOI model, the required inputs, the calculations, and the output are described. Outputs include the expected value of perfect information and the expected VOI obtained from sampling. The section also demonstrates how the VOI changes with changes in the state of knowledge prior to gathering the information, changes in the costs of errors, and changes in the quality of information.

\subsubsection{Decision Setting}

To illustrate the concepts and calculations involved in a VOI analysis, the following highly simplified example, based loosely on the organic-nitrate safety issue, is presented. All aspects of the model have been over-simplified to show details of the calculations. A realistic illustrative analysis of this safety issue is presented in Section 4.1 .

The moisture content of the tank waste is important to the organic-nitrate safety issue because wet waste will not sustain an organic-nitrate reaction. Suppose that some information is available on the moisture content of the waste in the tanks and a decision must be made from among the following three alternatives: 1) declare that the tank is safe based on its average moisture content being above $20 \%, 2$ ) take mitigative action on the tank, or 3 ) gather more information about moisture before deciding between 
alternatives 1 and 2 . The consequences of the decision depend on whether the moisture is actually above or below $20 \%$. Consequences of the decision include the cost of mitigation, the cost of gathering information, and the effects of falsely declaring a tank to be safe. In this example, it is assumed that all consequences can be expressed.in dollars, although they may account for an array of consequences.

\subsubsection{Structure of the VOI Model}

The formal structure of a VOI model is a decision tree (Raiffa 1968; Clemen 1991). The decision tree for our example is shown in Figure 3.1. This decision tree shows the sequence of actions (squares) and uncertain events (circles) that describe the decision setting. It also shows the criteria that are used to characterize the consequences of the combinations of actions and events. The decision tree is read from left to right. The path leading to any point in the tree is shown to the left of the point and is assumed to be determined. Paths to the right of any point show all subsequent possibilities, which are not yet determined. Decision or action nodes (squares) are under the control of the decision maker, who may choose which branch to follow. Event nodes (circles) are not under the control of the decision maker but are subject to uncertainty. A probability distribution represents the uncertainty about which branch will occur.

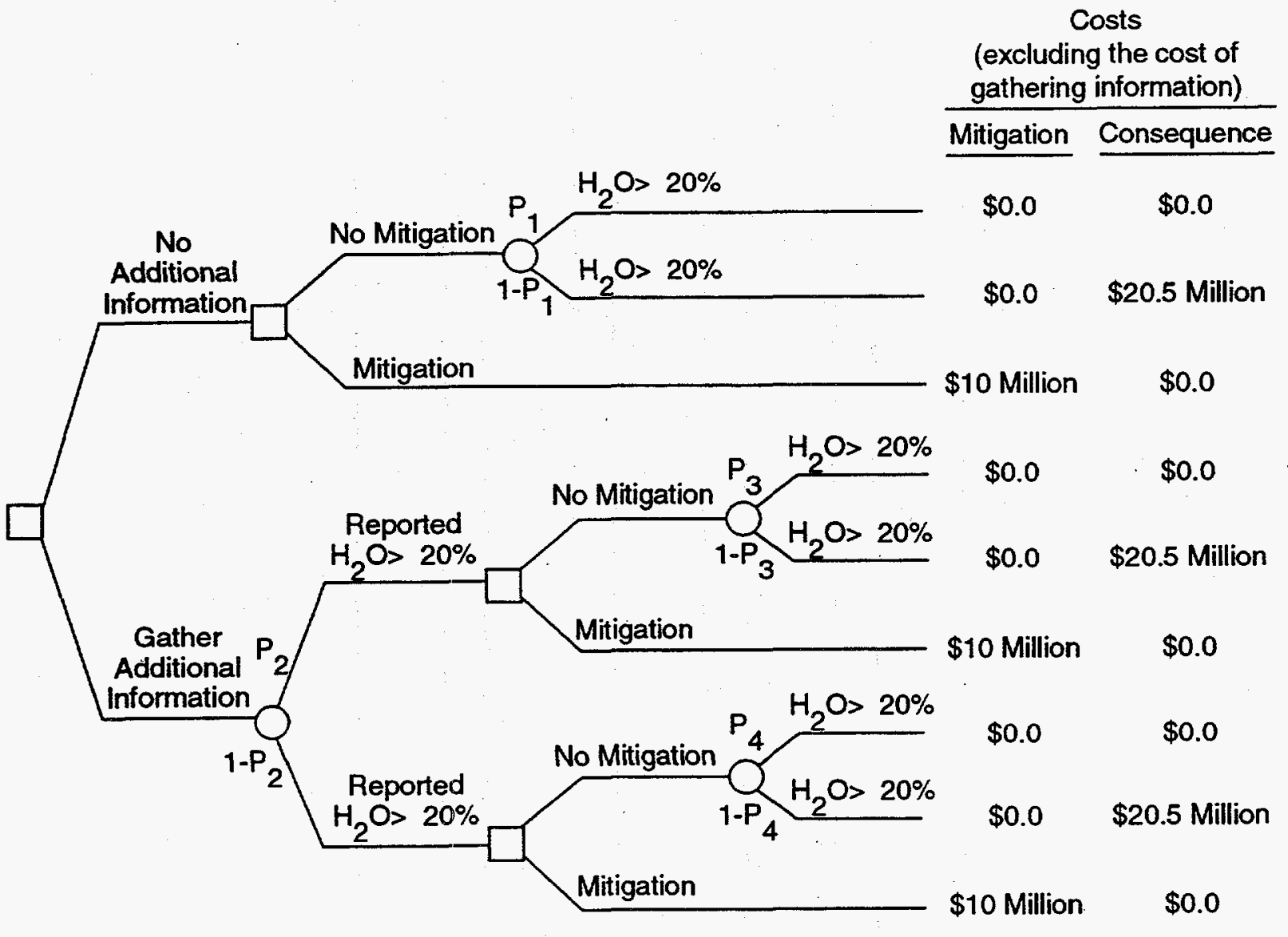

Figure 3.1. Illustrative VOI Model 
The first decision is whether to gather additional information, which is shown as the first square on the left. If no additional information is to be gathered, then the choice is made whether to mitigate. If the choice is made not to mitigate, then it is important whether the moisture level is above or below $20 \%$. This example assumes that the mitigation is completely effective, so that the original moisture level is no longer important once the mitigative action is taken.

If the choice is made to gather additional information, the next node on the path is an event that describes the results from the information-gathering activity. This is shown as a report that moisture is above or below $20 \%$. To simplify the language, we will simply call these two states "high moisture" and "low moisture." The next node encountered along either path is an action node showing the choice of whether to mitigate. As before, if the choice is made not to mitigate, then it is important whether the moisture level is above or below $20 \%$. This last event node refers to the actual moisture level, which contrasts with the earlier event node that contained the reported moisture level.

The attractiveness of each of the nine combinations of actions and events (paths through the tree) is characterized by its costs of mitigation, consequences, and information gathering. The example assumes that all of the consequences can be expressed as equivalent dollar values. Consequences include all attributes that represent the attractiveness of the paths through the tree (e.g., costs, health and safety, effects on cultural assets).

Associated with each uncertain event is its probability of occurrence. There are four probabilities specified in the tree:

- $\mathrm{p}_{1}=$ the unconditional probability that the actual moisture level is high

- $\mathrm{p}_{2}=$ the probability that the information gathering will report that the moisture level is high

- $\mathrm{p}_{3}=$ the conditional probability that the moisture level is actually high given that the report says that it is high

- $\mathrm{p}_{4}=$ the conditional probability that the moisture level is high given that the report says that it is low.

The last two probabilities account for both possible reporting errors, falsely reporting that moisture is high $\left(1-\mathrm{p}_{3}\right)$ and falsely reporting that moisture is low $\left(\mathrm{p}_{4}\right)$.

The decision tree model is processed using the calculation procedure known as "rollback." First, valuation measures (costs) are assessed for all nine paths through the tree, and all four probabilities are also assessed. Next, the tree is evaluated from right to left. The expected cost is determined for event nodes by summing the products of probabilities and costs for all of the node's branches. The expected cost at a decision node is the lowest expected cost from among the node's branches. This corresponds to choosing the alternative with the lowest expected cost. The process is repeated until expected values are determined for all nodes. Processing the tree in this manner provides a complete specification of the best decisions at all decision nodes and a determination of the VOI. The expected VOI is the difference 
between the expected value of the best decision without gathering the information and the expected value of the decision to gather information. This value depends on what is already known without gathering more information (which is represented by $\mathrm{p}_{1}$ ), the costs of the nine combinations of actions and events, and the diagnosticity of the information to be gathered (which is represented by $p_{3}$ and $p_{4}$ ). The following numerical examples illustrate this process.

\subsubsection{Value of Perfect Information}

Assume that the parameters are as follows: Based on previous information and prior to gathering the information under analysis, the probability that the moisture level is high $\left(p_{1}\right)$ is 0.95 . The cost of mitigation is $\$ 10$ million. The consequences of a moisture level that is low are equivalent to a cost of $\$ 20.5$ million if no mitigative action is taken. There are no costs to the consequences if moisture is high or mitigative action is taken. This assumes perfectly effective mitigation. These costs are shown at the ends of the paths in Figure 3.1. The best decision is to mitigate if the moisture level is low and to not mitigate if the moisture is high. Of course, the actual concentration is not known before the decision must be made, so there is a value to getting this information.

In the rollback calculation for the top portion of the tree, the expected value at the uncertain event node describing actual moisture level is

$$
(\$ 0.0) \times(0.95)+(\$ 20.5 \text { million }) \times(0.05)=\$ 1.025 \text { million }
$$

The expected value at the action node of whether or not to mitigate is

$$
\operatorname{Min}(\$ 1.025 \text { million, } \$ 10 \text { million })=\$ 1.025 \text { million }
$$

The conclusion from this calculation is that (ignoring for now the alternative of gathering additional information) the best choice is to not mitigate and to accept the risk. This choice results in an expected cost of $\$ 1.025$ million.

Using only the parameters given above, it is also possible to determine the expected value of perfect information (EVPI). This is the expected cost of making the decision without additional information minus the expected cost of making the decision after obtaining perfect information about moisture. The expected cost of making the decision without any additional information is calculated above as $\$ 1.025$ million. Perfect information would permit the best choice in either moisture condition; that is, perfect information about moisture would allow the decision-maker to choose to mitigate if the moisture were low and to avoid mitigation if the moisture were high. The expected cost of the decision under perfect information is the cost of no mitigation when moisture is high times the probability that it is high $\left(\mathrm{p}_{1}\right)$, plus the cost of mitigation when moisture is low times the probability that it is low $\left(1-\mathrm{p}_{1}\right)$ :

$$
(\$ 0.0) \times(0.95)+(\$ 10 \text { million }) \times(0.05)=\$ 0.50 \text { million }
$$


Thus, the EVPI is $\$ 1.025$ million - $\$ 0.50$ million $=\$ 0.525$ million. This value places an upper bound on the expected VOI that could actually be obtained, since no information can be better than perfect information. In this case, if additional information costs more than $\$ 525,000$, then it is not worth its cost.

\subsubsection{Value of Imperfect Information}

Of course, perfect information of the type analyzed above is impossible to obtain. What is more interesting is the value of the imperfect information that could be obtained from characterization. For this, we need to know the diagnosticity of the information, (i.e., how well the information will help distinguish whether the moisture level is above or below $20 \%$ ). One way to describe the diagnosticity of information is through conditional probability statements of the reported information, given the actual condition. Assume for this example that the diagnosticity of information that might be obtained from a particular characterization of tank contents can be described as a probability of 0.90 of receiving a report that the moisture level is high if it actually were high, and a probability of 0.95 of receiving a report that the moisture level is low if it actually were low.

The probability of the report given actual moisture is useful information, but it is not in the form required by the decision tree in Figure 3.1. What is needed for $p_{3}$ and $p_{4}$ are the probabilities of actual levels given the reports. The required probabilities can be calculated by applying Bayes' Theorem, which states that the probability of the actual level given the reported level is equal to the probability of the reported level given the actual level times the probability of the actual level divided by the probability of the reported level.

The probabilities of the reported levels given the actual levels are stated above as 0.90 and 0.95 . The probabilities of the actual levels are $p_{1}$ and $1-p_{1}$, stated above as 0.95 and 0.05 . The probability of a high reported moisture $\left(p_{2}\right)$ is the probability of a report of high given that moisture is actually high times the probability that moisture is actually high, plus the probability of a report of high given that moisture is actually low times the probability that moisture is actually low:

$$
\mathrm{p}_{2}=(0.90) \times(0.95)+(0.05) \times(0.05)=0.8575
$$

Similarly, the probability of a low reported moisture is the probability of a report of low given that moisture is actually high times the probability that moisture is actually high, plus the probability of a report of low given that moisture is actually low times the probability that moisture is actually low:

$$
(0.10) \times(0.95)+(0.95) \times(0.05)=0.1425
$$

Alternatively, this is calculated as $1-\mathrm{p}_{2}=1-0.8575=0.1425$.

Applying Bayes'Theorem gives the remaining probabilities required for Figure 3.1:

$$
\mathrm{p}_{3}=(0.90) \times(0.95) / 0.8575=0.855 / 0.8575=0.997
$$




$$
\mathrm{p}_{4}=(0.10) \times(0.95) / 0.1425=0.095 / 0.1425=0.6667
$$

With this information, we can calculate the expected value of the best decision conditional on each report, and the expected value of the decision to gather information. The rollback calculations for the bottom part of the decision tree in Figure 3.1 are as follows. If the report is that moisture is high, then the expected cost of no mitigation is

$$
(\$ 0.0) \times(0.997)+(\$ 20.5 \text { million }) \times(0.003)=\$ 0.06 \text { million }
$$

The expected cost of mitigation is $\$ 10$ million, so the better decision is no mitigation at an expected cost of $\$ 0.06$ million.

If the report is that moisture is low, then the expected cost of no mitigation is

$$
(\$ 0.0) \times(0.6667)+(\$ 20.5 \text { million }) \times(0.3333)=\$ 6.83 \text { million. }
$$

Again, the expected cost of mitigation is $\$ 10$ million, so the better decision is still no mitigation, but now the expected cost of the decision is $\$ 6.83$ million.

Combining these expected costs with the probabilities of the reports (through $\mathrm{p}_{2}$ ) gives an expected cost of the decision to gather information of

$$
(\$ 0.06 \text { million }) \times(0.8575)+(\$ 6.83 \text { million }) \times(0.1425)=\$ 1.025 \text { million }
$$

The expected value of this information is calculated by taking the difference between the expected cost without the information, which was calculated above, and the expected cost with the information. In this case, the expected VOI information is

$$
\$ 1.025 \text { million }-\$ 1.025 \text { million }=\$ 0.0 \text {. }
$$

This information is not worth anything. The decision would not change regardless of the report, so the expected cost is the same whether the information were obtained or not. Of course, this conclusion depends on the specific costs and probabilities, as illustrated below.

\subsubsection{VOI with Different Input Values}

This section shows how the VOI changes if the model inputs (prior knowledge, costs, and the diagnosticity of the information) are changed. All values shown in this section are purely illustrative to demonstrate the calculations in a VOI model.

Change Prior Knowledge. The VOI changes if we change the information that is known prior to gathering the information under question. For example, if the prior information were $p_{1}=0.75$ rather 
than 0.95 , and all of the costs and diagnosticities remained exactly the same as above, then the calculations would be as follows. The decision to gather no more information and to perform no mitigation would have an expected cost of

$$
(\$ 0.0) \times(0.75)+(\$ 20.5 \text { million }) \times(0.25)=\$ 5.125 \text { million }
$$

This is still less than the cost of mitigation ( $\$ 10$ million), so the better decision is still no mitigation. Perfect information would indicate a decision to mitigate with a 0.25 probability, so the expected cost of the decision with perfect information is

$$
(\$ 0.0) \times(0.75)+(\$ 10 \text { million }) \times(0.25)=\$ 2.50 \text { million }
$$

Thus, the expected value of perfect information is

$$
\$ 5.125 \text { million }-\$ 2.50 \text { million }=\$ 2.625 \text { million. }
$$

With the same diagnosticity of the information, the values of the required inputs to the tree in Figure 3.1 are now

$$
\begin{gathered}
\mathrm{p}_{2}=(0.90) \times(0.75)+(0.05) \times(0.25)=0.6875 \\
\mathrm{p}_{3}=(0.90) \times(0.75) / 0.6875=0.982 \\
\mathrm{p}_{4}=(0.10) \times(0.75) /(1-0.6875)=0.240
\end{gathered}
$$

Now, the expected cost of no mitigation if the reported average moisture is high is

$$
(\$ 0.0) \times(0.982)+(\$ 20.5 \text { million }) \times(0.018)=\$ 0.37 \text { million. }
$$

The expected cost of no mitigation if the reported average moisture is low is

$$
(\$ 0.0) \times(0.24)+(\$ 20.5 \text { million }) \times(0.76)=\$ 15.58 \text { million. }
$$

which is greater than the $\$ 10$ million cost of mitigation. Thus, the best decision is to not mitigate if the reported moisture is high and to mitigate if the reported moisture is low. This decision has an expected cost of

$$
(\$ 0.37 \text { million }) \times(0.6875)+(\$ 10 \text { million }) \times(0.3125)=\$ 3.38 \text { million }
$$

The expected value of the information is now $\$ 5.125$ million $-\$ 3.38$ million $=\$ 1.74$ million (rounded) for information of the same accuracy as in the first example. The difference here is that much the tank contents are known with less certainty before gathering additional information, so getting information could change the mitigation decision. 
Change Costs. The VOI is also sensitive to costs. Assume that the costs associated with no mitigation of a tank with a low moisture level are $\$ 66$ million rather than $\$ 20.5$ million. Further, assume that the prior knowledge of the tank's contents is the same as in the first numerical example, $p_{1}=0.95$. The expected cost of a decision to gather no additional information and to conduct no mitigation is now

$$
(\$ 0.0) \times(0.95)+(\$ 66 \text { million }) \times(0.05)=\$ 3.30 \text { million }
$$

which is still less than the cost of mitigation. In this case, the expected value of perfect information is

$$
\$ 3.30 \text { million }-\$ 0.50 \text { million }=\$ 2.80 \text { million. }
$$

If the prior probabilities and the diagnosticity of the information are the same as in the first example, then the values of $p_{2}, p_{3}$, and $p_{4}$ are the same as in the first example. That is, $p_{2}=0.8575, p_{3}=0.997$, and $\mathrm{p}_{4}=0.667$. The expected cost of no mitigation if the reported moisture is high is

$$
(0.0) \mathrm{x}(0.997)+(\$ 66 \text { million }) \mathrm{x}(0.003)=\$ 0.19 \text { million. }
$$

The expected cost of no mitigation if the reported moisture is low is

$$
(\$ 0.0) \times(0.667)+(\$ 66 \text { million }) \times(0.333)=\$ 22 \text { million }
$$

which is greater than the $\$ 10$ million cost of mitigation. Thus, the best decision is to mitigate if the reported moisture is low and to not mitigate if the reported moisture is high. This decision has an expected cost of

$$
(\$ 0.19 \text { million }) \times(0.8575)+(\$ 10 \text { million }) \times(0.1425)=\$ 1.59 \text { million. }
$$

The expected VOI is now $\$ 3.30$ million $-\$ 1.59$ million $=\$ 1.71$ million. Again, this is the same information that had an expected value of $\$ 0.0$ in the first example. The difference is that here the costs of an incorrect decision to not mitigate when moisture is low are much greater. This changes the decision based on the outcome of the report, even though the report is no more accurate than in the first example.

Staying with this example, it is instructive to see the effect of changes in the costs of mitigation. Suppose that mitigation had a cost of $\$ 1$ million instead of $\$ 10$ million and that all other parameter values were the same as in the previous paragraphs. The expected cost of a decision to gather no additional information and to not mitigate is still $\$ 3.30$ million. However, the cost of mitigation is now $\$ 1.0$ million, so the preferred choice in the absence of additional information is to mitigate. The expected value of perfect information is now

$$
\$ 1.0 \text { million - }((\$ 0.0) \times(0.95)+(\$ 1 \text { million }) \times(0.05))=\$ 0.95 \text { million }
$$


The availability of an effective, low-cost mitigative action reduces the value of perfect information from $\$ 2.80$ million to $\$ 0.95$ million. The cost of such a mitigative action places an upper bound on the VOI.

The value of the additional information provided by characterization is reduced as well. It is still better to mitigate if the reported moisture is low and to not mitigate if the reported moisture is high. The expected cost of not mitigating when the reported moisture is high is still $\$ 0.19$ million, but the decision to mitigate now costs only $\$ 1.0$ million. So, the expected cost of the decision to gather additional information is now

$$
(\$ .19 \text { million }) \times(0.8575)+(\$ 1.0 \text { million }) \times(.01425)=\$ 0.31 \text { million }
$$

So, the expected value of the information is: $\$ 1.0$ million $-\$ 0.31$ million $=\$ 0.69$ million. This compares with a VOI of $\$ 1.71$ million when the cost of mitigation is $\$ 10$ million.

Change Diagnosticity. As a final example, consider the effect on the VOI of changing the diagnosticity of that information. Assume that the prior information and costs are the same as in the first example, but that the diagnosticity of the information under analysis is different. In particular, assume that the conditional probability of a high reported moisture value given that it actually is high is 0.99 rather than 0.90 . Further assume that the conditional probability of a low reported moisture value given that it actually is low remains at 0.95 . Since the prior probabilities and the costs are the same as in the first example, $\mathrm{p}_{1}=$ 0.95 and the expected value of perfect information is the same as well, $\$ 0.525$ million.

The change in diagnosticity changes the values of the probabilities in Figure 3.1 as follows, using the calculations described previously:

$$
\begin{gathered}
\mathrm{p}_{2}=(0.99) \times(0.95)+(0.05) \times(0.05)=0.943 \\
\mathrm{p}_{3}=(0.99) \times(0.95) / 0.943=0.9974 \\
\mathrm{p}_{4}=(0.01) \times(0.95) /(1-0.943)=0.1667
\end{gathered}
$$

With these parameter values, the expected cost of not mitigating if the reported moisture is high is

$$
(\$ 0.0) \times(0.9974)+(\$ 20.5 \text { million }) \times(0.0026)=\$ 0.05 \text { million. }
$$

The expected cost of not mitigating if the reported moisture is low is

$$
(\$ 0.0) \times(0.1667)+(\$ 20.5) \times(0.8333)=\$ 17.08 \text { million, }
$$


which exceeds the $\$ 10$ million cost of mitigation. Thus, the best decision is to not mitigate if the reported moisture is high but to mitigate if the reported moisture is low. This decision has an expected cost of

$$
(\$ 0.05 \text { million }) \mathrm{x}(0.943)+(\$ 10 \text { million }) \mathrm{x}(0.057)=\$ 0.62 \text { million. }
$$

So the VOI is $\$ 1.02$ million $-\$ 0.62$ million $=\$ 0.40$ million. The VOI in the original example was $\$ 0.0$. This more diagnostic information is worth more.

\subsubsection{Expected Net Gain from Sampling}

As demonstrated in the previous section, the VOI depends on its diagnosticity. Generally, more diagnostic information is more valuable. In this sense, diagnosticity can be viewed as a measure of the quality of information. Usually, improved quality of information comes at a price. For example, a larger sample size reduces the sampling error, or a more costly sampling and analysis method reduces some other error component. A VOI analysis can determine the best information-gathering strategy through an examination of the expected net gain from sampling (ENGS). ENGS for any proposed characterization strategy is equal to its VOI minus its cost. The best choice is the method with the highest ENGS.

To examine the ENGS for different characterization strategies, start by calculating the VOI for each strategy. As described above, the VOI depends on the diagnosticity of the information as determined from the conditional probabilities of correct or incorrect reports. For example, the basic analysis in Section 3.1 .4 considers a strategy that provides a 0.90 probability of reporting that moisture is high if it is actually high, and a 0.95 probability of reporting that moisture is low if it is actually low. This same diagnosticity can be stated as the probability of incorrect reports. That is, there is a 0.10 probability of a report of low moisture if it is actually high, and a 0.05 probability of a report of high moisture if it is actually low.

This latter way of stating diagnosticity is analogous to the statistical terms "Type I" and "Type II" errors. A Type I error is to reject a true hypothesis. A Type II error is to accept a false hypothesis. If the hypothesis is that moisture is low, then the information described in the previous paragraph has a $5 \%$ chance of a Type I error and a $10 \%$ chance of a Type II error.

Errors in measuring continuous variables such as moisture are often stated as parameters related to the quantity being measured. The choice of the parameter might also be related to the functional form of the probability distribution of the variable. For example, if moisture were known or assumed to be distributed log normally, then a convenient way to state the uncertainty is by the relative standard deviation. Knowing the RSD of an information-gathering strategy allows one to calculate its Type I and Type II errors. An RSD of zero corresponds to perfect information and Type I and Type II errors of zero. As RSD increases, the probabilities of Type I and Type II errors increase, and the quality and value of information decreases.

Of course, the RSD of an information-gathering strategy will depend on the method of gathering the information and the specifics of the strategy, such as sample size. The method and specifics such as 
sample size will also determine the cost of the information-gathering strategy. One would expect that the cost would increase within a given method as its RSD decreased. The VOI can be calculated from the model as described above. The ENGS of the information-gathering strategy is its VOI minus its cost.

Table 3.1 illustrates an ENGS analysis for two information-gathering methods. This analysis uses the same prior probabilities and costs as in the basic analysis in Section 3.1.4. The table shows Type I and Type II errors as a function of RSD, the resulting VOI, the cost necessary to attain the RSD level by each of two methods, and the resulting ENGS for each combination of method and RSD.

The conclusion from this analysis is that the best strategy is to gather information using Method 1 at an RSD of 20\%. This method and degree of accuracy provides the highest ENGS $(\$ 166,000)$. ENGS drops off with less accuracy because the VOI drops more quickly than the cost. ENGS drops off with greater accuracy because the VOI rises more slowly than the cost. Method 2 is never recommended. ENGS is negative for all RSD values with Method 2.

\subsubsection{Estimation Approach: Introduction to Loss Functions}

The above description of the VOI approach may be characterized as the "decision" approach to VOI analysis. Decision analysts generally regard this decision approach as technically best. Its advantages are that it links the information directly to the decisions that need to be made and that it carefully analyzes the impact of information on the decision. Its disadvantages in the tank waste area are that there are many

Table 3.1. Expected Net Gain from Sampling (ENGS) Analysis

\begin{tabular}{|c|c|c|c|c|c|c|c||}
\hline & & & & \multicolumn{2}{c|}{ Method 1 } & \multicolumn{2}{c|}{ Method 2 } \\
RSD & Type I & Type II & VOI & Cost & ENGS & Cost & ENGS \\
\hline \hline 0 & Error & Error & thousands & thousands & thousands & thousands & thousands \\
\hline $5 \%$ & 0 & 0 & $\$ 525$ & - & - & - & - \\
\hline $10 \%$ & 0 & 0 & $\$ 525$ & $\$ 900$ & $-\$ 375$ & - & - \\
\hline $20 \%$ & 0.0062 & 0 & $\$ 466$ & $\$ 300$ & $\$ 166$ & $\$ 900$ & $-\$ 434$ \\
\hline $30 \%$ & 0.0478 & 0.0004 & $\$ 71$ & $\$ 200$ & $-\$ 129$ & $\$ 600$ & $-\$ 529$ \\
\hline $40 \%$ & 0.1060 & 0.0062 & $\$ 0$ & & & & \\
\hline $50 \%$ & 0.1590 & 0.0227 & $\$ 0$ & & & & \\
\hline $60 \%$ & 0.2020 & 0.0478 & $\$ 0$ & $\$ 100$ & $-\$ 100$ & $\$ 300$ & $-\$ 300$ \\
\hline $70 \%$ & 0.2380 & 0.0765 & $\$ 0$ & & & & \\
\hline $80 \%$ & 0.2670 & 0.1050 & $\$ 0$ & & & & \\
\hline $90 \%$ & 0.2890 & 0.1330 & $\$ 0$ & & & & \\
\hline $100 \%$ & 0.3090 & 0.1590 & $\$ 0$ & & & & \\
\hline
\end{tabular}


interlinked decisions, and that it is not always possible to model all the decisions in detail. An alternative and somewhat less sophisticated approach that uses most of the concepts of VOI and decision analysis is the estimation approach.

The estimation approach treats the estimate of the uncertain variable as a decision and specifies a loss function for mis-estimating the true parameter. In general, the loss function would be estimated in dollars by considering scenarios for making decisions using a wrong estimate. This approach puts the burden of modeling decisions and consequences into a simple loss function. Thus, the problem of understanding the detailed and interlinked decisions does not go away, it is simply cut short with a surrogate-the analyst's definition of how much the decision maker stands to lose by mis-estimating a tank waste variable. This approach is easier to use and probably easier to generalize, but the loss function is simply an approximation of the real expected losses and gains in a decision problem.

Realizing the advantages and disadvantages of the decision versus the estimation approach to assessing the value of characterization information, this report includes examples of both (see Sections 4.2.2 and 4.2.3). Both approaches share the qualitative analysis of decisions and information sources (Section 4.2.1) and the assessment of prior distributions as discussed in the next section.

\subsubsection{Elicitation of Selected Prior Distributions}

The characterization strategy will define the tank waste variables that are most likely to be important for disposal decisions. Independent of the overall approach taken to develop a tank characterization strategy, it is useful to quantify the uncertainty about these variables in the form of prior probability distributions.

Because these prior distributions are essential inputs into most subsequent analyses, it is very important that they be elicited with state-of-the-art procedures. An approach that is similar to the rigor of the NUREG 1150 (NRC 1989) uncertainty assessments should be used. This approach is described in Section 4.2.1 as it applies to tank waste characterization.

Prior probability distributions for the organic-nitrate safety issue analysis described above were estimated for each tank from analysis of past data about the tanks. Data included the history of waste inventories in the tanks, photographs of tank waste, and previous sampling and analysis of the tank contents. Statistical models were used to fit the parameters of interest for the analysis to the available data.

Constructing prior probability distributions can build on existing work. For example, McConville et al. (1995) provides a first-cut set of priors in the form of three-point approximations of probability distributions. In addition, substantial work exists on historical records, simulations, and actual samples. The main guidelines for this additional effort to construct prior probability distributions are to select the best experts and to conduct a formal elicitation. Otherwise, the results of this elicitation will merely be considered just another set of opinions. 


\subsection{Possible Outputs from a VOI Analysis}

The following are some of the possible outputs from a VOI analysis of safety issues. The particular outputs provided will depend on how the analysis is formulated and how the results are developed and analyzed. These remarks refer to VOI analyses that are performed on each tank. This section is written in the context of safety issues; similar outputs would result from a VOI analyses of disposal issues.

\subsubsection{Probability Distributions to Support Risk Analyses}

A VOI analysis of a tank safety issue can provide information that supports a risk analysis on the tank. This information is in the form of a probability that a certain value of a risk characteristic (e.g., radiation dose or toxic chemical exposure) will be exceeded. This can be compared with risk guidelines or thresholds.

This output is not part of the VOI analysis per se but is a byproduct of the probabilistic model of events and their consequences. To provide this output, the characteristics must be estimated as part of the input. For example, the radiation dose consequences must be estimated for each possible event in the VOI decision tree.

In some cases, the units of measurement will differ from those used in traditional risk analysis. For example, the risk analysis may require the radiation dose to the maximally exposed individual, but the VOI analysis may need the total person-rems of exposure. These cases require some procedure to estimate both inputs to support both types of analyses.

\subsubsection{VOI and Sampling Strategies}

Fundamental outputs from a VOI analysis are the expected value of perfect information (EVPI) and the expected value of sample information (EVSI). EVPI is the expected value of learning for certain the true state of an uncertain quantity that is modeled in the analysis. If several uncertainties are included in the model (e.g., uncertainty over a tank's total organic carbon [TOC] content and uncertainty over its moisture content), then EVPI can be calculated for each uncertain quantity and for each combination of quantities. Perfect information is usually unattainable; however, its value places an upper bound on the VOI that is attainable.

EVSI is the expected VOI with a specified diagnosticity. Diagnosticity is a measure of how likely it is that the information correctly specifies the actual value of the uncertain quantity. If more than one uncertain quantity is represented in the model (e.g., TOC and moisture), then diagnosticity expresses how diagnostic the information is of each uncertain quantity. Diagnosticity is represented in a VOI analysis as a conditional probability distribution of what the information will say conditional on each possible actual value of each uncertain quantity. EVSI can be calculated for actual or hypothetical information-gathering actions. These information-gathering actions are not limited to those involving sampling and analysis but 
could include such actions as analysis of historical data, laboratory experimentation, computer models and simulations, and theoretical research. The key is that each information-gathering's diagnosticity must be . specified in order to calculate its expected value.

Both EVPI and EVSI are usually expressed in dollars. This facilitates the comparison of the expected value of the information with the cost of the information, which is usually stated in dollars.

An EVSI can be calculated for several different information-gathering actions to determine the best course of action. For example, EVSI could be calculated for sample sizes of 1,2,3, and so forth that are analyzed for all or some of the uncertain quantities. Each information-gathering action represents a characterization strategy for the tank. It specifies what information is sought, how the information is to be obtained, and the sample size. Each EVSI is then compared with the cost of sampling to determine the optimal sample size, which is the sample size that has the greatest ENGS. The net gain from sampling is equal to the EVSI minus the cost of sampling.

A table of EVPI and EVSI or ENGS values for each tank and each sample size considered provides the basis for a strategy on tank sampling to support the particular safety issue being analyzed. First, if EVPI is less than the cost of sampling for any tank, then no sampling is justified for that tank. Sampling might be justified for tanks with EVPI greater than the cost of sampling, but ENGS must be considered. If ENGS is greater than zero for any actual sample size, then some sampling is justified. The optimal sample size is that with the highest ENGS. Since the diagnosticity of the sampling schemes depends on both the sample size and the characteristic for which the sample is taken (e.g., TOC, moisture, or both), the strategy defines both what to sample for and the size of the sample. Similar considerations apply for other types of information gathering.

This strategy applies strictly only to the first sampling action to be taken. If sampling in one tank provides information about other tanks, then the VOI in the other tanks will change after the first tank's information is known (see Section 3.2.4).

\subsubsection{VOI and Mitigation Strategies}

In a VOI decision analysis, information has value only if it has the possibility of changing an action or decision. For safety issues, this action is often one of mitigation. (The term "mitigation" is used broadly and includes actions to reduce the probability of adverse consequences as well as actions to reduce the magnitude of those consequences.) A VOI decision analysis will show the best mitigative action to take, including possibly no mitigation, in the absence of information and the best mitigative action to take conditional on each possible reported result from an information-gathering action. In all cases, "best" is determined by choosing the action or set of actions that minimizes the total expected cost or maximizes the expected utility, considering the probabilities of all consequences. Cost includes all adverse consequences, not just dollars. Expected values are also shown for each mitigation. Together, this information provides a recommended mitigation strategy for each tank. 
The mitigation strategy can be combined with the sampling strategy discussed under Section 3.2.2. For each tank, the sampling strategy indicates the optimal sample size and the mitigation strategy indicates the optimal mitigation, conditional on each reported outcome. The following is a hypothetical example of such a strategy: "Take a single rotary core sample from Tank X-000 and analyze it for TOC as a weight percentage. If the analysis reports that TOC is less than X\%, then perform no mitigation; if the reported TOC is between $\mathrm{X} \%$ and $\mathrm{Y} \%$, then control ignition sources; if the reported TOC is greater than $\mathrm{Y} \%$, then install a moisture monitoring and control system in the tank." As with the sampling strategy, the mitigation strategies can be arranged in a table that summarizes the best strategy for each tank.

\subsubsection{Sequential Sampling Strategies}

A sequential sampling strategy is one whose later information-gathering actions depend on earlier results. For example, the best sample to take second might depend on the results of the first sample. The most tractable sequential analysis of sampling strategies for tank safety VOIs is to rerun the analysis based on reported results as they are received.

At an extreme, the analyses could all be rerun after each individual core sample is taken. First, ENGS is calculated for a single sample from each tank. Then a single sample is chosen from the tank with the highest ENGS. The information from the sample is then used to update the probability distributions for all tanks, and new ENGS values are calculated for a single sample from each tank. The next sample is taken from the tank with the highest revised ENGS, and the process is repeated.

Because it is difficult to gain access to a tank for sampling, and because there is considerable delay between the time a sample is taken and the time that it is analyzed, it is impractical to rerun the VOI analyses after each sample. A close approximation is to recalculate ENGS values for each sample strategy after the receipt of each report. An example of this sequential strategy is to begin with the calculations described in Section 3.2.2 and choose the strategy with the highest ENGS, which might be to take three core samples from a particular tank. After the results from the analysis of these samples are known, all probability distributions could be updated to account for the information, the ENGS values recalculated for sampling strategies on all other tanks, and the tank with the highest ENGS chosen for the next sampling.

If time delays for the analysis of samples require that the initial sampling strategy specify the first several tanks to be sampled, a further approximation is needed. Since probability distributions of tank contents are estimated by first forming groups of similar tanks, an initial specification in a sequential strategy might be to specify the strategies with the highest ENGS for each group of tanks. Results from the analyses of these tank samples would then be used to update the probability distributions of the remaining tanks, and the new values of ENGS would be used to specify the next group of tanks to be sampled. An analogous approach would be used to specify a sequential sampling strategy for information-gathering actions other than tank sampling. 


\subsubsection{Informal Strategies Across Tanks}

Performing a series of VOI analyses on all tanks allows one to develop informal strategies that apply to all tanks. For example, the detailed quantitative analyses of each tank might show that, for a large group of tanks, information about surface moisture is much more valuable than information about TOC. In addition, it might be determined that this information can be obtained from auger samples at a much lower cost than taking a core sample, which provides information about both TOC and moisture. These observations may lead to the informal conclusion that auger samples analyzed for moisture should precede the more expensive core samples, and that all ENGS values should be recalculated after the results from the auger samples are reported.

A variety of such informal analyses are possible. They are usually performed by analyzing tables of values and strategies developed as described in the previous three sections.

\subsubsection{Characterization Plans}

Characterization plans can be developed from VOI decision analyses for all uncertain tank variables for safety issues. First, for each safety issue, show what information in needed about each tank. For example, the organic-nitrate safety issue might need information about the concentrations of total organic carbon and moisture in the waste. The ferrocyanide safety issue might need information about the concentrations of ferrocyanide, oxidants, and moisture in the waste. The flammable gas safety issue may need information about the concentration of flammable gas in the headspace and the composition of the waste. All safety issues might need information about the consequences of a tank dome failure or a HEPA filter failure.

Each VOI analysis will model one or more uncertain quantities, which define what information should be sought by an information-gathering action. The quantities also help to identify possible means for gathering the information. For example, a drop bottle may be sufficient to estimate the concentration of gases in the headspace, an auger sample may be sufficient to estimate the concentrations of identified chemicals and moisture in the waste's surface, core samples may be needed to estimate the total waste composition, and computer models and simulations may be needed to estimate the consequences of a dome failure.

EVPI calculations show how valuable it is to obtain perfect information about each uncertain quantity to support each safety issue in each tank. For most tanks, the EVPI to support any safety issue is less than the cost of obtaining and analyzing a core sample from the tank. In these cases, it should be concluded that core sampling from the tank is not justified by the need to resolve the safety issue for the tank. The combined value of perfect information to support all safety issues for a tank is approximated by the sum of EVPIs for the tank across safety issues. Again, most tanks will show values too low to justify core sampling to resolve the issues for the tank.

The sum of EVPIs across tanks within a single safety issue places an upper bound on the value of obtaining information about an uncertain quantity or quantities that supports resolution of the safety issue. 
If this value is below the cost of actually obtaining the information by some means, then that means of collecting information is not justified by the safety issue. For example, if the sum of EVPIs across all tanks for a safety issue is less than the cost of one core sample, then core sampling is not justified by its support of that safety issue. The sum of EVPIs across all tanks and all safety issues similarly provides an upper bound on the value of all types of information to support safety issues. This last value is likely to be a very large bound, and therefore is of little value in setting a characterization plan.

For tanks for which the EVPI does not rule out information gathering, EVSIs for particular information-gathering schemes provide the information needed to determine the best way to sample. In general, the best first information-gathering action is the one with the highest ENGS. If, however, the action provides information about several uncertain quantities, then ENGS for a single quantity underestimates the net value of the action. In addition, if a similar action provides information about more than one quantity, then ENGS may also underestimate the net value of the action. For example, if a core sample could be analyzed for multiple quantities for a total cost that is close to the cost of obtaining the sample and analyzing it for a single quantity, then ENGS calculated for a single quantity underestimates the value of the sample.

The case of multiple types of information from essentially the same action can be handled by a simple adjustment to the EVSI values calculated and tabulated as discussed in Section 3.2.2. The adjustment is to consider the total value and total cost of the action. In the case of a small incremental cost to analyze a core sample for multiple quantities, the procedure adds the EVSIs across information categories and uses the total cost for the analyses. For example, if several safety VOIs for a tank produce EVSIs for different sample sizes, then the total EVSI is the sum of the EVSIs (this is approximate if multiple analyses used the same uncertain quantity, such as moisture). The cost of taking the sample and analyzing it for all quantities is subtracted from this sum to determine its adjusted ENGS. If this procedure is repeated for all sample sizes, the optimal sample size is the one with the highest revised ENGS.

Revised ENGS values could be tabulated in the manner discussed in Section 3.2.2. The best first characterization action is the one with the highest revised ENGS. The form of this action might be to take three core samples from Tank U-000 and analyze them for TOC, moisture, and ferrocyanide concentrations and provide a list of all chemical constituents.

Such a table will show ENGS values for other tanks and other actions as well. A characterization plan to support safety issues could be developed by choosing the entries with the highest revised ENGS values. A refinement of this plan would consider the effects of sequential sampling as discussed in Section 3.2.4. An examination of the table might also lead to the discovery of informal strategies of the nature discussed in Section 3.2.5.

\subsection{Incorporation of Risk-Based Constraints}

The terms "safe" and "clean" contain implicit assumptions and requirements regarding acceptable risk. Hanford tank waste constitutes a significant "hazard" (i.e., it poses the potential for harm to human health, the environment, and socio-cultural practices and values). Programmatic goals, such as meeting technical 
objectives, schedule, or budgetary targets may be "harmed" or upset by unacceptable conditions or events encountered in waste management or disposal. Incorporation of risk constraints into VOI models poses several issues:

- How can risk be addressed in a way that supports decision-making based on the "best" balance of cost, risk, and benefit (risk-based decision-making)?

- What types of risk (what kinds and degrees of harm to what receptors) should be addressed in a particular decision context, and what are the proper measures of those risks?

- How can the types and measures of risk be integrated into VOI analysis to ensure that proper value is assigned to information that effectively supports decisions and outcomes that meet risk acceptance goals?

This section describes the risk constraints that govern the TWRS Program and how risk will be used in the VOI analysis. Risk constraints measures are discussed in Harper et al. (1996).

\subsubsection{Risk Dimensions and Constraints}

The risks that should be addressed in risk-based decision making arise from several sources:

- Stakeholder values

- Laws and regulations

- Scientific and technical standards and practices

- Policies and guidance documents.

Both the risk categories identified here and the measures themselves are based on Keeney and von Winterfeldt (1996), WHC (1995), the environmental regulatory framework (including the Tri-Party Agreement) (Ecology 1996), and other Hanford sources, including comments submitted by stakeholders over the years.

\subsubsection{The Basic Risk Categories}

Four basic risk categories (and associated measures) emerge from an examination of the sources: The Human Health Category is concerned with public and worker health effects associated with routine and accident conditions and includes multi generational effects.

The Environmental Category is concerned with contamination of and physical impacts on environmental media (air, water and soil) and living resources.

The Socio-Cultural Category is concerned with community quality of life and impacts to cultural resources. 
The Programmatic Category is concerned with impacts on mission (including achievement of specified goals and implementation of technical options), cost, and schedule.

\subsubsection{Key Measures of Risk}

Key risk measures such as annual radiological dose expressed in rem/year constitute the scales used to express risk (essentially in terms of consequences) within the basic risk categories. A total of 20 Hanford risk measures (summarized in Table 3.2) describe the four basic risk categories. These individual measures are presented in Appendix A. The key risk measures encompass both routine operations and

Table 3.2. Individual Risk Measures (see Appendix A for more detail)

\begin{tabular}{||l|l||}
\hline CATEGORY: PUBLIC/WORKER HEALTH & $\begin{array}{l}\text { CATEGORY: SOCIO-CULTURAL QUALITY } \\
\text { OF LIFE }\end{array}$ \\
\hline \hline H1. Short-term public/worker radiological dose & S1. Community quality of life \\
\hline $\begin{array}{l}\text { H2. Short-term public/worker non-radiological } \\
\text { exposure. }\end{array}$ & S2. Tribal quality of life \\
\hline H3. Annual worker radiological dose & S3. Intra- and Intergenerational equity \\
\hline H4. Annual public radiological dose & S4. Impacts to cultural resources \\
\hline H5. Annual population radiological dose & CATEGORY: PROGRAMMATIC \\
\hline $\begin{array}{l}\text { H6. Lifetime individual cancer and non-cancer } \\
\text { risk }\end{array}$ & P1. Penalties for noncompliance \\
\hline H7. Multi generation risks & P2. Shutdown costs \\
\hline \hline CATEGORY: ENVIRONMENTAL IMPACTS & P3. Response and repair costs \\
\hline E1. Environmental disturbance during & P4. Mission impacts \\
\hline E2. Ecotoxicity and habitat impacts & P5. Interprogram and Sitewide impacts \\
\hline Emen response or remedial action & P6. Cost of stakeholder non-involvement \\
\hline
\end{tabular}


accidents. Accident risks are an issue for waste management and cleanup operations; post-closure and cleanup states imply the achievement of risk goals without further management/corrective activity or opportunity for accidental release.

Most of the key measures are directly extracted from Site standards and practices, individual regulations and/or widely recognized Site values. However, a few additional measures are included which are typically required by the decision makers or the public and which, if omitted, have the potential to change decisions. Stakeholder review of the measures, risk acceptance levels, and especially the monetary valuation of the consequences is important. Assignment of equivalent dollar values to disparate consequences is difficult and often contentious. Choosing the right unit(s) of impact is challenging. For example, conversion of the value of a human life or a non-lethal health effect into dollar amounts is counterintuitive to many stakeholders. Without active stakeholder participation in this process, the ultimate acceptance of these measures and proposed actions will remain uncertain.

\subsubsection{Acceptable Levels of Risk}

The 20 key measures describe the landscape of risk, but the answers to the questions of what constitutes safe and clean depend upon the identification of acceptable risk levels within these measures. Acceptance limits can be expressed as discrete points on the risk measures scale, or in some cases, as qualitative statements of consequences of interest.

Risk Acceptance Constraints and the Regulatory Framework. Acceptable risk levels are often prescribed by law. For instance, for Public/Worker Health Measure $1(\mathrm{H} 1)$, the risk acceptance limit prescribed by law for routine annual worker radiological dose is $5 \mathrm{rem} / \mathrm{yr}$. The acceptable annual radiological dose from nuclear facilities/activities for the public is $100 \mathrm{mrem} / \mathrm{yr}$. Other measures, even if derived from legal authority, have no prescribed risk acceptance level. For example, natural resource damage during environmental cleanup activities (under CERCLA) is prohibited where not explicitly excused by a resource commitment under the National Environmental Policy Act, but specific compliance limits for types and degrees of natural resource damage are not specified in the regulations. It is important to note that some environmental laws permit the use of alternative risk:levels where there are good reasons for departing from the generally prescribed standards and where it can be persuasively demonstrated that the alternative approach will achieve the underlying goals of the law.

Risk Acceptance Constraints at Hanford. Human health risk acceptance constraints for routine/normal operations are well developed in the regulatory framework and operative policies and standards at Hanford. However, routine risk acceptance levels for environmental, socio-cultural and programmatic risk are not well institutionalized at Hanford, and accident risk acceptance levels are not well addressed in any of the primary risk categories. The Safety Analysis Manual (WHC-CM-4-46, Rev. 4), which does contain accident risk acceptance criteria for human radiological and chemical risk, is currently under review. 
Utility of Risk Acceptance Constraints in VOI and Risk-based Decision-Making. There are several reasons to identify or determine risk acceptance levels for each risk measure:

- In addition to understanding the risks associated with any proposed action, the decision makers (and stakeholders) need to understand whether those risks fall within prevailing risk acceptance levels or whether exceeding those levels is appropriate to achieve the best cost/risk/benefit outcome.

- Negotiation of alternatives to compliance with usual legal (or conventional) standards depends upon explicitly showing the gap between "strict" compliance and the proposed alternative risk levels. (This also means that the analysis of alternatives must include relevant risk measures expressed in appropriate units, e.g., annual worker radiological dose in rem/yr).

- When converting consequences to costs, each measure must be assigned its own cost of achieving and of exceeding the risk acceptance level, especially if a single event can lead to several adverse outcomes.

- Risk acceptance levels will, in many cases, define "break-points" in cost/risk curves because exceeding an acceptance level may require additional technical assessment, negotiation with regulators or other stakeholders, additional mitigation measures, or significant programmatic adjustments. This is particularly true if the risk acceptance level is a regulatory standard.

- The set of risk acceptance levels essentially constitutes an envelope for safe (active containment) and clean (passive or no containment) operations. The safe-and-clean envelope integrates the various categories and dimensions of risk around a (negotiable) decision space, and supports normalization of disparate types of impacts. Comparisons and tradeoffs among human health and environmental impacts, for instance, is challenging due to lack of agreement about relative worth. By defining conceptually similar constraint levels for each measure (such as what routine consequences are acceptable, or at the other extreme, what severe consequences are acceptable given $10 \mathrm{E}-6$ probability), the acceptance points on the individual risk measure scales are automatically aligned. The translation of envelope-defining metrics to a common currency (such as equivalent dollar value in the VOI approach) provides a basis for calibration of both the acceptance levels and the equivalent dollar values.

\subsubsection{The Use of Risk Acceptance Constraints in VOI Applications}

Risk acceptance constraints are used in VOI analysis to: 1) ensure that the structure of the analysis reflects the relevant consequences and attendant risks associated with a particular problem to the extent necessary to make a decision, and 2) provide, as a byproduct of the analysis, probabilities that selected risk acceptance constraints will be exceeded (see Section 3.2.1). VOI analysis and associated risk analyses can therefore be integrated to describe the cost, risks and benefits associated with alternative actions and to show where those alternatives stand in terms of risk acceptability. 
The risk acceptance constraints will be used in the VOI approach in three ways (see Figure 3.2):

- Preliminary screen to eliminate clearly unacceptable alternatives

- Framework to discriminate among alternatives for a particular decision

- Posterior screen to evaluate preferred technical options for acceptability and to determine whether to implement an alternative, negotiate flexibility in the constraints, or analyze other alternatives.

Preliminary Screen. Risk acceptance constraints will be used to screen postulated alternatives and ensure that the starting points for analysis conform as much as possible to the risk acceptance envelope. However, because the objective of VOI analysis is to find the best overall alternative, it is important to

\section{Anterior Screen \\ (low precision)}

Are any of the proposed options clearly unacceptable?
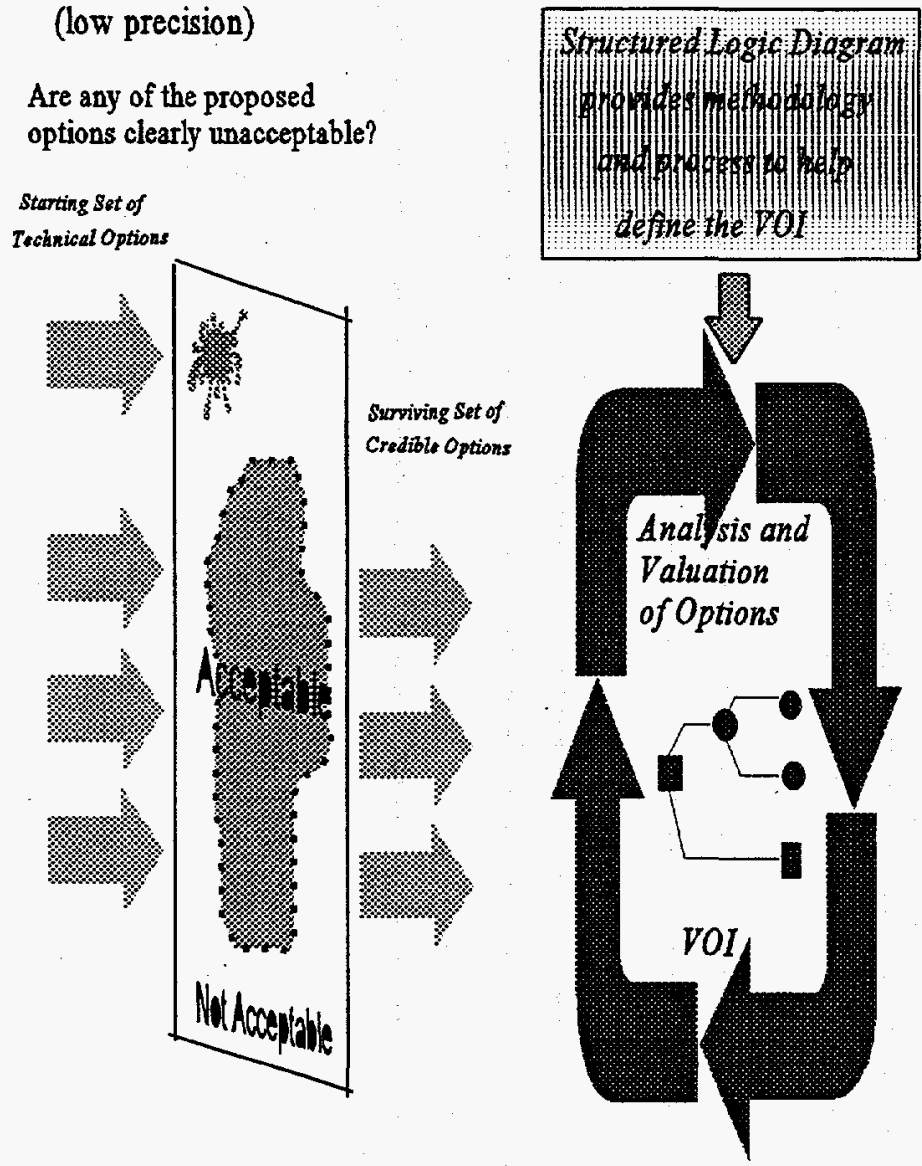

Posterior Screen

(higher precision)

Does the preferred option meet

all risk constraints, or does it suggest that acceptable risk levels should be negotiated toward a balanced cost-risk-benefit solution?

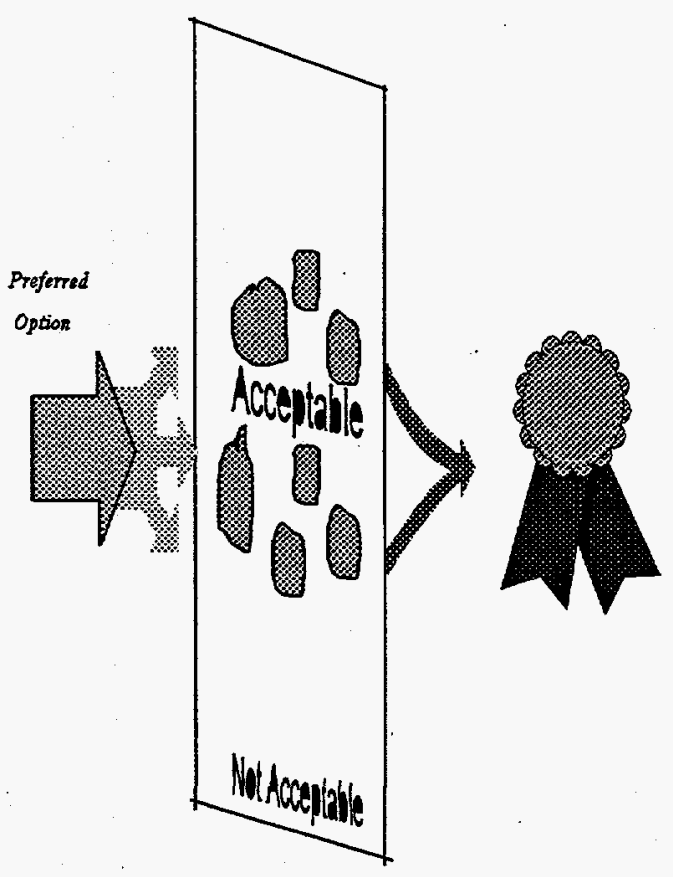

Risk Measures Used for:

- Developing the Problem Statement

Figure 3.2. The Use of Risk Acceptance Measure/Constraints 
allow for analysis of alternatives that might fall outside of the envelope in order to provide a basis for understanding the cost of meeting constraints and also to allow the best overall solution to emerge based on tradeoffs among costs, risks, and benefits.

Framework for Alternative Evaluations. A limited set of risk constraints is used to frame the problem so that alternative actions can be evaluated in terms of overall acceptability, and to ensure that the risks that drive the decision and that materially contribute to discrimination among alternatives are captured. The limited set of risk constraints used in any particular VOI application should be selected from the complete set with several objectives in view:

- Selected measures should reflect the significant risks associated with the decision (action) being analyzed.

- Selected measures should represent the whole problem.

- Measures should be included only to the extent necessary to achieve enough certainty to make a good decision.

- Selected measures should be traceable to the technical, legal, and Site values basis.

VOI analysis will be constructed using the set of measures that achieves the above objectives. Down selection will be achieved by using measures only from relevant risk categories and by judicious use of proxy or combined measures from the master set. The preferred alternative will reflect the relevant risk implications in a way that is clear and understandable to the stakeholders and permits practical assessment against the entire risk envelope.

Posterior Screen. The preferred alternative that emerges from VOI analysis can be compared with the risk envelope to determine whether it is likely to be acceptable across all the relevant risk acceptance constraints. The preferred alternative might meet all constraints (clearly acceptable); it might meet many or most constraints (good fit) and therefore suggest focus areas for tradeoffs and negotiation; or the screen may suggest that other alternatives should be reevaluated. An iterative approach can be taken using VOI and the risk acceptance envelope to reach the best solution (i.e., the best cost/risk/benefit outcome that can be achieved in light of technical and budgetary issues and through an appropriate stakeholder involvement process). 


\subsection{VOI Applications}

\subsection{VOI Analysis for Safety Issue}

This section illustrates how VOI decision analysis methods can be used to develop a characterization strategy as it relates to safety issues. The organic-nitrate safety issue was chosen to illustrate the methods because a significant amount of work had been completed on a VOI analysis of this safety issue by November 1995. The analysis as it existed at that point in time was used as the basis for the illustration; however, some of the inputs were updated and several parts of the previous analysis extended to meet the needs of this illustration. As this report was being prepared, the organic-nitrate safety issue was being analyzed further for preparation of a Safety Analysis Report (SAR) that is expected to be published late in 1996. The analyses and conclusions presented here are based on earlier data and may be superseded by the SAR. The methods, however, remain valid.

\subsubsection{Organic Nitrate Problem Description}

The organic-nitrate safety issue in the Hanford single-shell tanks (SSTs) arises from the fact that many of the high-level wastes in these tanks contained organic compounds when last processed, and sampling tank wastes has shown significant concentrations of organic compounds in some tanks. Based on processing history and assay data, all tanks are presumed to have significant quantities of oxidizers, including sodium nitrate and sodium nitrite. Recent laboratory studies show that organic concentrations below about $5 \mathrm{wt} \%$ (dry basis) are very unlikely to support propagating reactions, but concentrations of $7 \mathrm{wt} \%$ can support potentially violent reactions in heated, dry material. Thus an accurate assessment of organic concentration, moisture level, and temperature status is basic to the assurance of interim (pre-retrieval) safe storage in these tanks.

Organic materials were used in several applications in the separations of nuclear materials at the Hanford Site, and many of the waste types generated included organic materials. These included ethylenediaminetetraacetic acid (EDTA), $\mathrm{N}$-hydroxyethylenediaminetriacetic acid (HEDTA), sodium citrate, sodium acetate, normal paraffin hydrocarbons (NPH), tributyl phosphate (TBP), and hundreds of miscellaneous compounds used in small-scale applications (Strachan et al. 1993). An estimated average chemical composition of these organic materials (Turner 1993) approximates sodium acetate. These waste materials have been degraded by radiolytic and chemical attacks in the waste tanks (Babad et al. 1993) but still retain significant potential fuel value.

The presence of these organic materials in the waste tanks is of concern because almost all of the wastes are rich in $\mathrm{NaNO}_{3}$ and $\mathrm{NaNO}_{2}$. Substantial effort has been expended to remove moisture from most tanks, and several tanks contain wastes with significant decay heat. Together, these factors could create conditions favorable for an organic-nitrate reaction. 
Concern for potential organic-nitrate reactions has resulted in an organic tank safety program at Westinghouse Hanford Company (WHC), and several SSTs have been placed on an organic tanks Watch List based on historical records (Babad and Turner 1993).

The organic-nitrate safety issue can be summarized as follows: An initiator such as a spark or lightning might ignite the organic waste in a tank, and this waste might burn. The burning might increase the pressure in the tank to the point where a HEPA filter fails or the tank dome failures. The HEPA filter failure or dome failure would allow the contents of the tank to be released to the environment.

The chain of events leading to the release of waste material to the environment is uncertain. There is uncertainty about whether an initiator could ignite the waste and whether the waste will burn. There is uncertainty about whether the burning waste will raise the pressure sufficiently to cause a HEPA filter failure or a dome failure. Characterization can provide information about some of these uncertainties.

A key uncertainty is whether the waste, if ignited, will burn. Webb et al. (1995) describes the conditions under which the organic-nitrate reaction will continue to burn, as shown in Equation 4.1.

$$
\text { \% TOC }>4.5+0.17\left(\% \mathrm{H}_{2} \mathrm{O}\right) \text { and } \% \mathrm{H}_{2} \mathrm{O}<20
$$

where \% TOC is the wt\% of TOC and $\% \mathrm{H}_{2} \mathrm{O}$ is the wt\% moisture. The first condition states that perfectly dry waste with greater than $4.5 \%$ TOC will burn. The required TOC concentration increases linearly at a rate of $0.17 \%$ per $1 \%$ of moisture until moisture reaches $20 \%$. The second condition states that waste with greater than $20 \%$ moisture will not burn.

Waste that will burn is called reactive waste. The part of the tank contents that is of interest for the organic-nitrate reaction is the part containing reactive waste. Burning reactive waste is of interest if it leads to HEPA filter failure or dome failure. Whether these events will occur depends on the pressure increase in the tank due to the burning. The pressure increase is related to the amount of reactive waste, as described below (see Damage Thresholds).

Reactive waste will lead to an adverse event only if it is ignited. Some ignition sources, such as lightning, will ignite any reactive waste. Other ignition sources, such as small sparks, will ignite reactive waste only if that waste is dry (less than $5 \mathrm{wt} \%$ moisture). We need to know the amount of reactive waste to determine the likelihood and seriousness of an organic-nitrate reaction. We also need to know how much of the reactive waste is dry ( $<5 \%$ moisture) and how much is damp (5\%-20\% moisture). The amounts of dry and damp reactive waste define our state space for tank contents.

Two damage thresholds are of interest for the organic-nitrate reaction: the threshold for a HEPA filter failure at $0.2 \mathrm{~atm}$ overpressure, and the threshold for a dome failure at $1.0 \mathrm{~atm}$ overpressure. The 
reactive volume needed to reach these pressures depends on the tank headspace volume. The thresholds for damage are calculated for each tank according to Equation 4.2.

$$
\mathrm{P}_{2} / \mathrm{P}_{1}=(1+\mathrm{aR} / \mathrm{H}) \times[1+(\mathrm{cR} / \mathrm{H}) /(1+\mathrm{bR} / \mathrm{H})]
$$

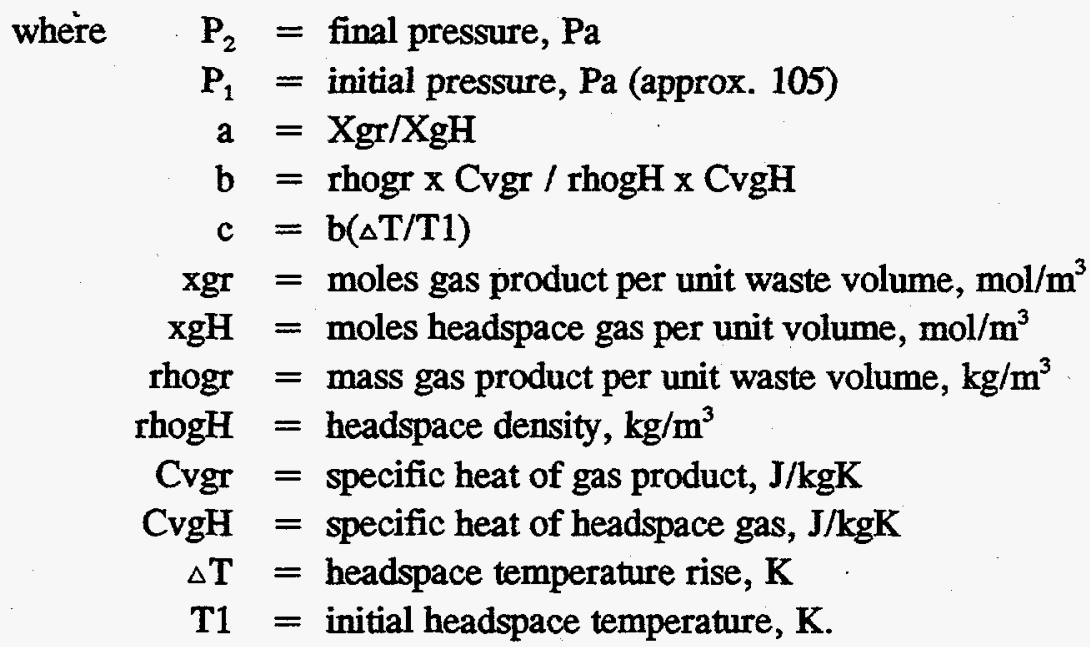

The parameters $a, b, c$, and $\triangle T$ depend on the TOC and moisture content of the reactive waste. All of the parameters are determined given the waste composition. By specifying the damage state pressure ratio $P 2 / P 1$, which is 1.2 for HEPA filter failure and 2.0 for dome failure, $R$ can be computed for each of the damage thresholds on a tank-by-tank basis. Results for HEPA failure are almost exactly $1 / 5$ the value for dome failure in all cases. The ratio $\mathrm{R} / \mathrm{H}$ (reactive volume to headspace volume) is $5 \times 10^{-4}$ for dome failure and $1 \times 10^{-4}$ for HEPA filter failure. In general, dome failure requires a reactive volume between 1 and $2 \mathrm{~m}^{3}$, and HEPA filter failure requires between 0.2 and $0.4 \mathrm{~m}^{3}$. The values of RHEPA and Rfailure for each tank are shown in Appendix B.

Shape of Reactive Waste. Since waste variations are more likely to occur vertically than horizontally due to waste layering and liquid draining, a pancake shape is reasonably conservative. For this analysis, we assume that the reactive waste is shaped like a pancake that is $10 \mathrm{~cm}$ deep. (A very thin pancake will not support a propagating reaction due to heat losses.)

Location of Reactive Waste. A review of Hanlon (1995) reports determined that the average waste volume in the $75-\mathrm{ft}$ diameter SSTs was $1,018 \mathrm{~m}^{3}$ with average waste depth of 2.5 meters. There are 25 different places that one could locate a $10-\mathrm{cm}$ pancake through this waste depth; therefore, the probability that the $10-\mathrm{cm}$ pancake of reactive waste was at the surface would be 0.04 , if it were randomly located. However, the reactive waste was relatively dry and the moisture gradient in the tank waste was dry near the surface and wet near the bottom. Thus it was more likely to find the reactive waste at the surface. We assumed a probability of 0.29 that the pancake of reactive waste was at the surface. This location was important because energy sources were more likely to be at the surface. 
Energy Sources and Probability of Ignition. The probability of ignition was estimated by multiplying an estimation of the frequency that energy sources existed in a tank by an estimate of the probability that the energy source would be co-located with reactive waste. Energy source frequencies were taken from Webb et al. (1995). The probability that the energy source was co-located with reactive waste was estimated using geometric analysis and assumptions regarding the shape of the reactive waste and the shape of the energy source. Probabilities of ignition were estimated for each energy source. These probabilities were then added to obtain an estimate of the probability of ignition by any source.

The analysis considered the following energy sources: lightning, small sparks, welding sparks and hot slag, core drilling upsets (from overheated drill bits), and vehicle gasoline fires. Lightning arcs to the waste causes high local temperatures, which would ignite reactive waste. Cowley and Stepnewski (1994) indicate that an arc to the waste surface (from equipment suspended just above the surface) would dissipate much of its energy in a circle $10 \mathrm{~cm}$ in diameter. Lightning current would also travel down a piece of equipment, such as a thermocouple tree, and arc to the waste at a point of high electrical resistance. These arcs could occur at any waste depth, and they would strike the pancake of reactive waste.

Small sparks could be produced by shorting electrical wires, dropped light bulb filaments, and other minor activities. We assumed that small sparks would not penetrate the waste, so they could only ignite reactive waste located at the surface.

Welding sparks are created in larger numbers than the small sparks. They may shower down into the tank through a riser or cascade overflow line.

The core drill upset was similar to lightning in that it could occur at any waste depth. The drill is most likely to overheat if the cooling purge gas flow fails while drilling through hard, dry saltcake. We assumed that the hot drill bit would find the pancake of reactive waste. The heated region around the drill bit is small, so the core drill acts as a point source at the surface of the waste. The frequency for core drill overheating was estimated considering the interlocks in place to prevent such scenarios. (Core drills are fitted with nitrogen bit cooling systems with shutdown interlocks if cooling fails, but operators have overridden the interlocks. The likelihood of overriding the interlocks was reflected in the estimated probability of ignition by core drilling upset.)

Burning gasoline from a vehicle might enter the tank through a broken riser or pit drain. The burning gasoline hits the waste surface and causes ignition. Alternatively, the gasoline might enter the tank, vaporize into a cloud of flammable gas, and ignite the waste. We assumed that the burning gasoline pool is 2 meters in diameter. The burning pool heats waste only at or near the surface. The frequencies for the energy sources are shown in Table 4.1.

Ignition frequencies for the energy sources shown in Table 4.1 were used, together with assumptions about the shape and location of the reactive waste, to estimate the probability of ignition. Table 4.2 summarizes these estimates. Lambda-dry is the estimated annual probability that the given amount of dry reactive waste would ignite and burn. Lambda-wet is the estimated annual probability that the given amount of damp reactive waste would ignite and burn. 
Table 4.1. Ignition Frequencies for Energy Sources

\begin{tabular}{||l|l|}
\hline Energy Source & Yearly Ignition Frequency \\
\hline Lightning & $1 \times 10^{-5}$ \\
\hline Small Sparks & $3 \times 10^{-3}$ \\
\hline Welding Sparks and Hot Slag & $1 \times 10^{-2}$ \\
\hline Core Drilling Upsets & $1 \times 10^{-4}$ \\
\hline Vehicle Gasoline Fires & $1 \times 10^{-4}$ \\
\hline
\end{tabular}

Table 4.2. Estimated Probabilities of Ignition for Dry or Damp Reactive Waste

\begin{tabular}{|c|c|c|}
\hline Reactive Volume, $\mathrm{m}^{3}$ & Lambda-dry, annual & Lambda-wet, annual \\
\hline 0.1 & $5.8 \times 10^{-5}$ & $1.72 \times 10^{-6}$ \\
\hline 0.2 & $7.81 \times 10^{-5}$ & $2.27 \times 10^{-6}$ \\
\hline 0.5 & $1.28 \times 10^{-4}$ & $3.69 \times 10^{-6}$ \\
\hline 1.0 & $1.99 \times 10^{-4}$ & $5.85 \times 10^{-6}$ \\
\hline 2.0 & $3.27 \times 10^{-4}$ & $9.88 \times 10^{-6}$ \\
\hline 5.0 & $6.79 \times 10^{-4}$ & $2.13 \times 10^{-5}$ \\
\hline 10 & $1.23 \times 10^{-3}$ & $3.97 \times 10^{-5}$ \\
\hline 20 & $2.285 \times 10^{-3}$ & $7.56 \times 10^{-5}$ \\
\hline 35 & $3.76 \times 10^{-3}$ & $1.23 \times 10^{-4}$ \\
\hline 50 & $3.91 \times 10^{-3}$ & $1.39 \times 10^{-4}$ \\
\hline 75 & $3.91 \times 10^{-3}$ & $1.39 \times 10^{-4}$ \\
\hline 100 & $3.91 \times 10^{-3}$ & $1.39 \times 10^{-4}$ \\
\hline
\end{tabular}

Mitigative Actions. Mitigative actions on the reactive waste or the ignition sources to reduce the probability of an organic-nitrate reaction that would result in a blown HEPA filter or a failured tank dome were 1) no action, 2) moisture monitoring and control system (MMCS), 3) flush and pump, 4) control ignition sources, and 5) waste retrieval and transfer.

MMCS was estimated to cost $\$ 500,000$. During the year of its installation, MMCS was estimated to double the probability of an adverse event. After installation, MMCS was estimated to have the effect of 
changing dry reactive waste into damp reactive waste; thus the estimated probabilities of ignition for dry reactive waste dropped to those of damp reactive waste.

Flush and pump was estimated to cost $\$ 3$ million. During the year of its installation, flush and pump was estimated to double the probability of an adverse event. After installation, flush and pump would render all waste nonreactive. Thus the estimated probabilities of ignition dropped to zero.

Control ignition sources was estimated to cost $\$ 100,000$. Controlling ignition sources was estimated to reduce the probability of igniting damp reactive waste by an order of magnitude immediately.

Waste retrieval and transfer was estimated to cost $\$ 20$ million. It would reduce the probability of an event to zero immediately.

\subsubsection{VOI Model for Organic-Nitrate Safety Issue}

Model Structure. The VOI model is the decision tree shown schematically in Figure 4.1. The primary structure of the model provides the value of obtaining information about a tank's TOC and moisture content. A secondary feature of the model is to indicate the preferred mitigation under each condition of information. In the course of determining these values and preferred decisions, the model also determines various measures of risk, the expected cost, and the probability of consequences for each action. The results of running the model for each tank can be combined to provide estimates over all tanks.

This model follows standard decision tree conventions, as explained in Section 3.0. Decision or action nodes, which are under the control of the decision maker, are shown as squares. Event nodes, which are subject to uncertainty, are shown as circles. The structure is read from left to right as a sequence of actions and events that represent the situation. At any point in the tree, everything to the left of the point is determined and everything to the right is yet to be determined. The complete tree for Figure 4.1 is too large to display on the page. In general, the complete tree is generated by attaching each node to every branch emanating from the previous node. The exception is that the node, Reported Tank Contents, is not attached to the previous branch, No Additional Information. The complete decision tree contains 2730 branches. This tree is processed for each specification of information quality; that is, each particular information-gathering scheme (e.g., a particular sample size) requires a complete specification and processing of this tree.

The first decision is whether to gather more information. If more information is to be gathered, the probability distribution over what the information will report about the tank contents is required. This information is summarized by 13 regions of the state space, labeled $A$ through $\mathbf{M}$. The state space is represented by the amount of dry reactive and damp reactive waste, as described in Section 4.1.1 and shown in Figure 4.2.

Whether information is sought or not, there is an opportunity to take mitigative action. The five actions described above are possible: no action, moisture monitoring and control system (MMCS), flush and 


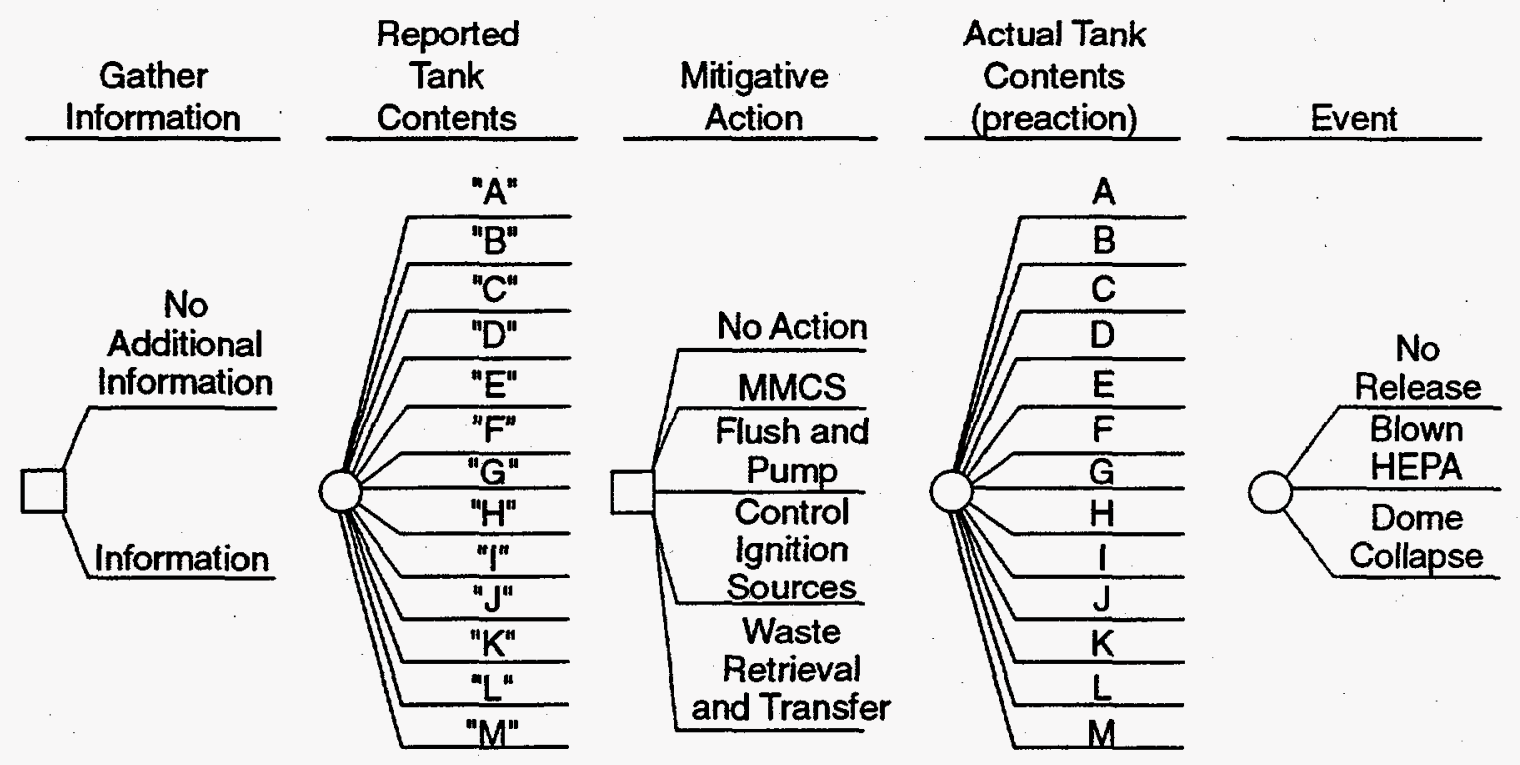

SG96100412.7

Figure 4.1. Decision Tree for the Organic-Nitrate Safety Issue

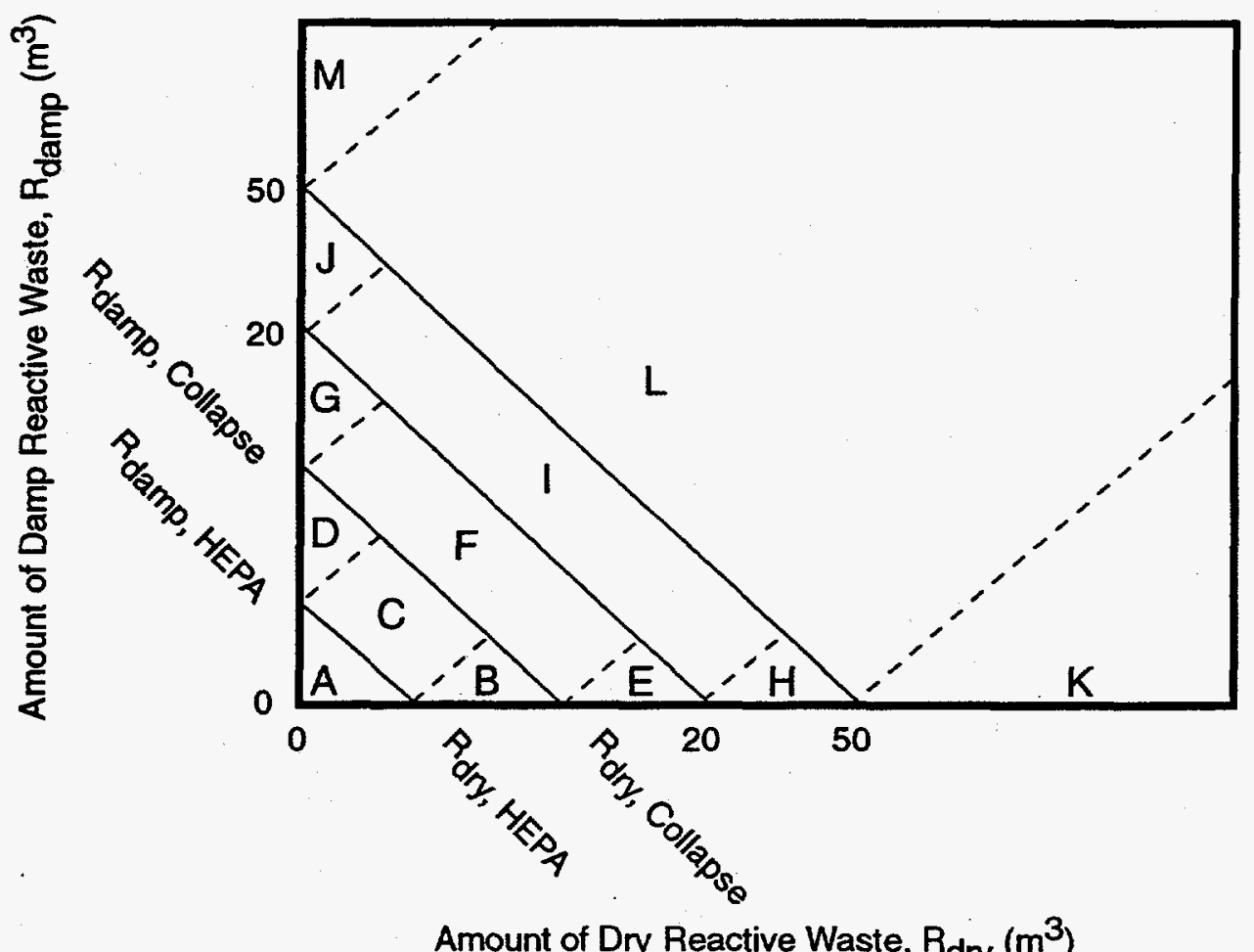

SG96100412.8

Figure 4.2. Representation of Tank Contents 
pump, control ignition sources, and waste retrieval and transfer. In cases where information is sought, the choice of the action is conditional on the reported tank contents. Where no information is sought, the mitigation is specified before learning anything more about the contents of the tank.

Following the action, an event node describes the uncertainty about the tank's contents in terms of 13 regions of the amounts of dry reactive waste and damp reactive waste (see Figure 4.2). The regions were chosen to correspond to the threshold amounts needed for adverse events and to provide a good approximation of the entire range of amounts possible. Region $A$ corresponds to an amount of reactive waste below the threshold needed to blow a HEPA filter. Regions B, C, and D correspond to amounts needed to blow a HEPA filter. They differ in the split between dry and damp reactive waste. The other regions are all above the amount necessary to cause a dome failure if ignited. In the model, a discrete point was chosen to represent each region. Midpoints along the Rdry axis were chosen to represent Regions B, E, and H. Midpoints along the Rdamp axis were chosen to represent Regions D, G, and J. Midpoints were chosen to represent Regions C, F, and I. The origin represented Region A, and larger amounts represented Regions K, L, and $M$.

The final node is an event node that describes the uncertain events. Three events represent the range of possibilities: tank dome failure, blown HEPA filter, or no release of the tank's contents.

Model Inputs. Inputs to the model included estimates of the costs of mitigative actions and event consequences and the probability distributions over information reports, tank contents, and events. For this analysis, 30-year costs and probabilities were used. Costs represented the attractiveness of each path, and they included several attributes. Direct dollar costs incurred in the path were one attribute. Direct dollar costs might arise from gathering the information, taking a mitigative action, or cleaning up land contaminated by an event. Events could also have other consequences, such as exposure of workers and the general public to radiation or toxic chemicals, or the degradation of cultural assets near the Hanford Site. All of these criteria were represented in the cost of each path. For the VOI analysis, these criteria were combined into a single measure and expressed as an equivalent dollar amount. Such an expression facilitated the comparison of alternatives and the determination of the best information-gathering decision.

The other inputs to the VOI decision tree model were probability distributions. Moving from right to left in Figure 4.1, the Event node contained the probability distribution over the three possible events conditional on the mitigative action and actual tank contents. The mitigative action influenced the probability that the waste would ignite. The tank contents influenced both the probability that the waste would ignite and the probability that burning waste would produce an event. If tank contents were in Region B, $C$, or D, burning waste would result in a blown HEPA filter. If tank contents were in Region $E, F, G, H$, $\mathrm{I}, \mathrm{J}, \mathrm{K}$, or $\mathrm{L}$, burning waste would result in the failure of the tank's dome. If the contents were in Region A, burning waste would result in no release.

The Actual Tank Contents node contains the probability distribution over the 13 regions of the amounts of dry and damp reactive waste. This probability distribution is conditional on reported tank contents. If no information is gathered, the prior probability distribution over contents is used. Prior probability 
distributions were estimated for each tank from analyses of past data about the tanks. Log normal distributions were estimated for the TOC and moisture of each tank, based on available information about the tank and groups of tanks. Monte Carlo simulations were used on these distributions to estimate the volumes of dry and damp reactive waste in the tank. These distributions were summarized as probability distributions over the 13 tank contents regions. The prior probability distributions for the 65 saltcake tanks used in analyses in this report are shown in Appendix B.

The Mitigative Action node contains the five actions described in Section 4.1.1. Mitigative actions might be taken after learning the reported tank contents. Mitigative actions other than No Action involve a cost and influence the probability that the tank waste will be ignited. These features are described above.

The Reported Tank Contents node contains a probability distribution over the reports that would be obtained from information gathering. This is calculated from statements of the diagnosticity of information. The diagnosticity of information is stated in the model as a set of conditional probability distributions. These are the probability distributions over reported tank contents conditional on actual tank contents. Tank contents are defined by the 13 regions of dry and damp reactive waste. The reports were assumed to be in the same form as the representation of the contents. For example, it was assumed that reports would be that the tank contents were in Region A, Region B, and so forth.

The diagnosticity of information was combined with the prior probability distributions over tank contents using Bayes' Theorem, as described in Section 3.0. This application of Bayes' Theorem provided two kinds of probability distributions needed by the model: the distribution over Reported Tank Contents and the distribution over Actual Tank Contents conditional on reported contents.

Diagnosticity is a way to express the quality of information. Perfect information is perfectly diagnostic in determining the actual tank contents. It is a set of conditional probability distributions with probabilities of 1.0 of having the same reported region as actual region. Less diagnostic information has a lower probability of receiving a correct report and a higher probability of receiving an incorrect report.

Specific Input Values. The total cost (considering all attributes) of the event of a blown HEPA filter was estimated at $\$ 2$ million. The total cost of a dome failure was estimated at $\$ 100$ million if the amount of reactive waste were less than $20 \mathrm{~m}^{3}, \$ 500$ million if the amount of reactive waste were 20 to $50 \mathrm{~m}^{3}$, and $\$ 1$ billion if the amount of reactive waste were greater than $50 \mathrm{~m}^{3}$. The estimated thresholds for a blown HEPA filter and dome failure, which define some of the tank content regions, varied by tank and are shown in Appendix B as are the prior probability distributions over tank contents, which also varied by tank. Ignition probabilities for different volumes of dry and damp reactive waste and different mitigative actions are given in Section 4.1.1; event probabilities conditional on the amount of burning reactive waste are discussed in Section 4.1.1.1.

Output Calculations. Output is calculated by "rolling back" the tree from right to left, as described in Section 3.0. At each event node, the expected cost of the node is calculated as the sum of the products of probabilities and costs over all of the branches emanating from the node. At each action node, the expected 
cost is the lowest expected cost from among all of its branches. Continuing this process produces an expected cost at every node in the tree. At the initial node, Gather Information, the expected values on its two branches, No Additional Information and Information, are of interest. The expected VOI is the difference between the expected cost without gathering information and the expected cost with the information. If the information modeled is perfect information, then VOI is the expected value of perfect information (EVPI). The VOI is compared with the cost of the information to determine whether the information is worth gathering. For sample information, the VOI minus the cost of sampling is known as the expected net gain from sampling (ENGS). If ENGS is positive, the information is worth its cost. If ENGS is negative, the information is not worth its cost. If several information-gathering choices are being analyzed (e.g., relating to different sample sizes), the VOI decision model will recommend the choice with the highest ENGS.

Another output from the model is the designation of the best mitigation strategy (i.e., the mitigation strategy with the lowest expected cost). The model shows, for each reported tank content and for the "no information" case, the mitigation strategy with the lowest expected cost, which is the recommended mitigation strategy. The VOI calculation assumes that this strategy will be followed.

\subsubsection{Organic-Nitrate Model Results}

The results from running the VOI decision model for the organic-nitrate safety issue are at three levels: results for individual tanks, results for all tanks taken together, and the resulting characterization strategy. Results from individual tanks include the expected value of perfect information, the expected value of sample information for any given sample scheme, the optimal mitigation strategy, and risk analyses. Results shown in this report are based on the information that was developed during the fall of 1995 and are intended to illustrate the methodology with realistic data. The results reported here are not definitive; the organic-nitrate safety issue continues to be analyzed and new data are being produced even as this report goes to press.

Results for Individual Tanks. Results for individual tanks were calculated by following the procedure described in Section 4.1.1.1. Inputs were estimated for all parameters in the model, and the VOI decision tree was rolled back to determine the expected VOI and the optimal mitigation strategy. The results of applying this procedure with the inputs for perfect information described above and in Appendix B for one tank, TX-102, are shown in Table 4.3.

The expected cost from rolling back the portion of the tree emanating from the No Information branch was \$1.94 million to take the mitigative action of installing a MMCS. Recall that cost was a summary measure of all adverse effects, not just dollar costs. The expected value was calculated as described in Section 4.1.1.1 as the sum of the products of probabilities times costs, sometimes referred to as the mathematical expectation. Because of the large number of branches in the organic-nitrate VOI decision tree, space does not permit a complete description of the calculation of the expected cost; a complete description for a much simpler tree is given in Section 3.0. 
With perfect information, the expected cost was $\$ 0.86$ million. The expected cost was lower with information because the information allowed mitigations that were best for the tank contents. These mitigations are shown at the bottom of Table 4.3. If the reported contents were in Region A, then, because the information was perfect, one would know that the actual contents were in Region A. Since Region A produced no release of the tank contents even if it were ignited, it required no mitigation. By knowing that the tank's contents were in Region A, one could avoid the expense of installing the MMCS, which was needed in the absence of information. Similarly, the expected cost of taking no action was less than the expected cost with a mitigative action if the contents were in Region B, Region C, or Region D. If the contents were in Regions E, F, H, or I, the best mitigative action would be to install an MMCS. If the contents were in Regions G, J, or M, the best mitigative action would be to control ignition sources. If the contents were in Regions $\mathrm{K}$ or $\mathrm{L}$, the best mitigative action would be to flush and pump the tank's contents.

Table 4.3. Results for a Single Tank

\begin{tabular}{|c|c|}
\hline Tank Identifier & $\mathrm{TX}-102$ \\
\hline Expected cost without information & $\$ 1.94$ million \\
\hline Expected cost with perfect information & $\$ 0.86$ million \\
\hline Expected value of perfect information & $\$ 1.08$ million \\
\hline Best mitigation without information & MMCS \\
\hline Reported Tank Contents (Region) & Best Mitigation with Reported Contents \\
\hline "A" & No Action \\
\hline “B” & No Action \\
\hline "C" & No Action \\
\hline "D" & No Action \\
\hline “E” & MMCS \\
\hline "F" & MMCS \\
\hline “G” & Control Ignition Sources \\
\hline "H" & MMCS \\
\hline "I" & MMCS \\
\hline "J" & Control Ignition Sources \\
\hline "K" & Flush and Pump \\
\hline "L" & Flush and Pump \\
\hline "M" & Control Ignition Sources \\
\hline
\end{tabular}


As with the case of no information, the expected cost was calculated as the sum of the products of probabilities times costs. The best mitigation given each possible report is the tank's optimal mitigation strategy.

The expected value of perfect information (EVPI) is the difference between the expected cost with perfect information and the expected cost without information. The EVPI was calculated as $\$ 1.94$ million minus $\$ 0.86$ million, or $\$ 1.08$ million. Although perfect information about the tank's contents is unattainable, the EVPI provides an upper bound on the value of any information that could be obtained about the contents.

The expected value of perfect information depends on all parameters in the model, including the costs of mitigation, the costs of consequences, and the prior knowledge of the tank's contents. The costs were essentially the same for all of the tanks, but prior knowledge of the contents varied considerably from tank to tank, as shown in the prior probability distributions in Appendix B. This had a dramatic effect on the EVPI calculated for each tank. For example, in Tank BX-110 the prior probability distribution had a 0.999 probability of contents in Region A, a 0.001 probability of contents in Region $\mathrm{D}$, and zero probability of contents in any other region. The EVPI for Tank BX-110 was zero. Its contents were known almost with certainty without any additional information, and the little uncertainty that was present would not change the best mitigative action. This contrasts with the prior probability distribution for Tank TX-102, which had considerable probability of tank contents in any region.

Other tanks had prior probability distributions like U-107, which had probabilities of contents in Regions A, D, G, J, or M. Region A had insufficient waste to cause a release of the tank's contents. The other regions contained only damp reactive waste, and the only uncertainty was the amount. EVPI for Tank U-107 was only $\$ 0.02$ million. Without information, its best mitigation was to control ignition sources, which was judged to be very effective with damp reactive waste. Perfect information would only change this decision and avoid costs of $\$ 100,000$ if the contents were in Region A or Region D. There was only a 0.212 probability that the contents would be in Regions A or D.

For tanks with very low EVPI, further analysis of the value of less-than-perfect information is unnecessary. However, for tanks with large EVPI, a further analysis can point the way to the best characterization decision. Recall that EVPI for Tank TX-102 was \$1.08 million. One might next inquire which is more valuable, information about the amount of TOC or information about moisture. This is especially important if different methods of sampling could be used to provide information about one characteristic but not the other. For example, an auger sample might provide good information about moisture but little information about the amount of TOC. Perfect information about the amount of TOC only is represented by a diagnosticity that differentiates perfectly among regions. with different amounts of TOC but is undiagnostic about regions with different moistures but the same TOC. Running the VOI decision model with this diagnosticity produced an expected value of perfect information about the amount of TOC of $\$ 0.33$ million. Similarly, the expected value of perfect information about moisture only is represented by a diagnosticity that differentiates perfectly among regions with different moistures but is undiagnostic about regions with different amounts of TOC. The expected value of perfect information about moisture was $\$ 0.78$ million. This result is displayed graphically in Figure 4.3. 


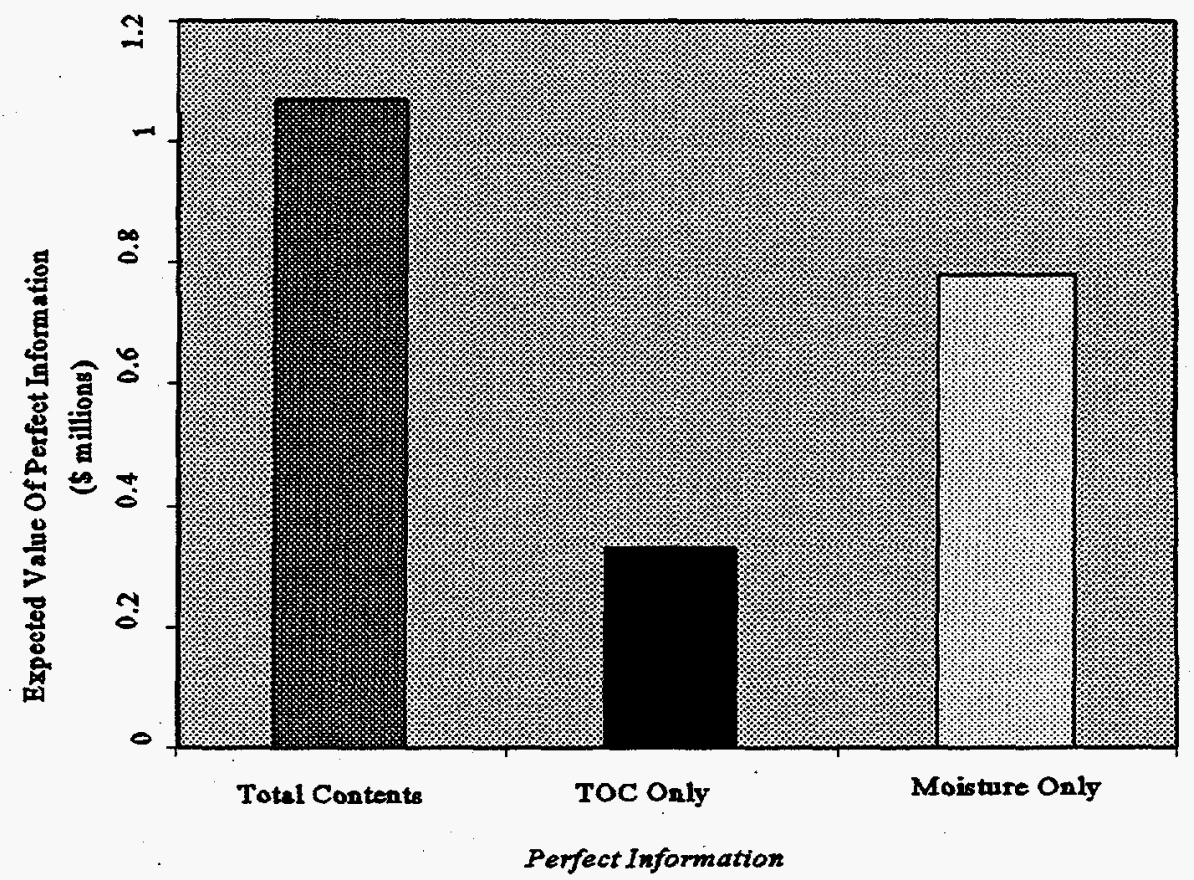

Figure 4.3. Expected Value of Perfect Information for the Organic-Nitrate Safety Issue for Tank TX-102

The EVPI about moisture and amount of TOC varied by tank, as did the relationship of these values to the EVPI. For some tanks, all of the EVPI was provided by the expected VOI about the amount of TOC. For others, perfect information about the amount of TOC provided only $2 \%$ of the EVPI. For some tanks, most of the EVPI was provided by perfect information about moisture; for others, information about moisture had no value.

The VOI decision model also indicated the optimal mitigation strategy for each type of information. These strategies took the mitigative action that minimized the expected cost conditional on each information report. These strategies were different for different tanks because they depended on the prior probability distributions.

The analysis of the value of less-than-perfect information can be taken another step to examine how the expected VOI changes as the diagnosticity changes. One such analysis examines how the VOI on the amount of reactive waste in the tank changes as the quality of the information changes. Figure 4.4 shows how the expected VOI on the amount of reactive waste in the tank decreases as RSD increases. Results are shown for four tanks: two with high values of perfect information about amount of TOC (S-105 and TX-102) and two with intermediate values of perfect information about amount of TOC (U-109 and U-107). Value does not drop at all as RSD increases from 0.0 to 0.2 . For high-valued tanks, the expected 


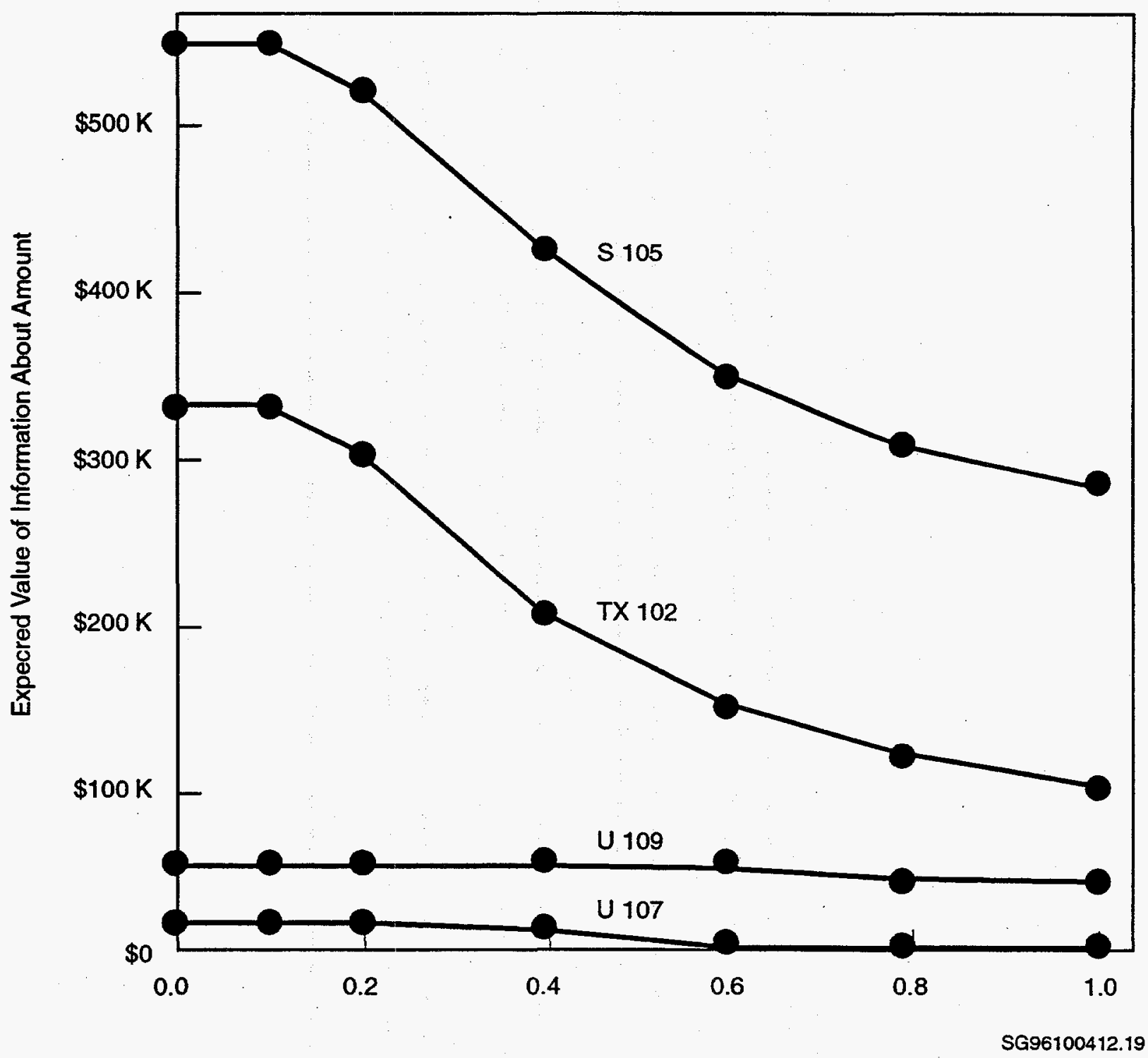

Figure 4.4. VOI Versus Quality of Information

VOI drops most quickly between RSD values of 0.2 and 0.8 ; above 0.8 , the value flattens out. These shapes are specific to the individual tanks. For Tank U-109, the expected VOI drops very little over the range of RSD from 0.0 to 1.0 .

The VOI decision model also provides information that can be compared with risk-based constraints. As an additional feature, the VOI decision model is a risk analysis model. It can provide probability distributions over consequences or events in addition to the expected values of information and the optimal mitigation strategy. For example, the model can provide single-year and multi-year estimates of the probability of the adverse events, blown HEPA filter and collapsed dome, conditional on the information and mitigation strategy. Table 4.4 shows a partial list of these results for Tank TX-102 under conditions of no information and perfect information. 
Table 4.4. Summary Results for Tank 241-TX-102

\begin{tabular}{|c|c|c|c|c|c|c|c|}
\hline \multirow[b]{2}{*}{$\begin{array}{c}\text { Reported } \\
\text { Tank } \\
\text { Contents }\end{array}$} & \multirow[b]{2}{*}{ Best Mitigation } & \multicolumn{3}{|c|}{ Probability of Dome Failure } & \multicolumn{3}{|c|}{ Probability of HEPA Filter Failure } \\
\hline & & First Year & Later Years & 30-Year & First Year & Later Years & 30-Year \\
\hline No Info & $\operatorname{MMCS}^{(2)}$ & $8.11 \mathrm{E}-04$ & $6.44 \mathrm{E}-05$ & 2.67E-03 & $5.28 \mathrm{E}-06$ & $5.94 \mathrm{E}-07$ & $2.25 \mathrm{E}-05$ \\
\hline “A" & No Action & 0 & 0 & 0 & 0 & 0 & 0 \\
\hline "B" & No Action & 0 & 0 & 0 & $1.92 \mathrm{E}-04$ & 1.92E-04 & $5.76 \mathrm{E}-03$ \\
\hline "C" & No Action & 0 & 0 & $\mathbf{0}$ & $1.24 \mathrm{E}-04$ & $1.24 \mathrm{E}-04$ & $3.72 \mathrm{E}-03$ \\
\hline "D" & No Action & 0 & 0 & 0 & $5.65 \mathrm{E}-06$ & $5.65 \mathrm{E}-06$ & $1.69 \mathrm{E}-04$ \\
\hline "E" & MMCS & $2.61 \mathrm{E}-03$ & 4.23E-05 & $3.84 \mathrm{E}-05$ & 0 & 0 & 0 \\
\hline "F" & MMCS & $1.44 \mathrm{E}-03$ & 4.23E-05 & $2.66 \mathrm{E}-03$ & 0 & 0 & 0 \\
\hline$" G^{\prime \prime}$ & Control Ignition & $4.23 \mathrm{E}-06$ & $4.23 E-06$ & $1.27 \mathrm{E}-04$ & 0 & 0 & 0 \\
\hline "H" & MMCS & $7.52 \mathrm{E}-03$ & $1.23 \mathrm{E}-04$ & $1.11 \mathrm{E}-02$ & 0 & 0 & 0 \\
\hline "I" & MMCS & $4.04 \mathrm{E}-03$ & $1.23 \mathrm{E}-04$ & $7.59 \mathrm{E}-03$ & 0 & 0 & 0 \\
\hline "J" & Control Ignition & $1.23 \mathrm{E}-05$ & $1.23 \mathrm{E}-05$ & $3.69 \mathrm{E}-04$ & 0 & 0 & 0 \\
\hline "K" & Flush and Pump & $7.82 \mathrm{E}-03$ & 0 & $7.82 \mathrm{E}-03$ & 0 & 0 & 0 \\
\hline "L" & Flush and Pump & $7.82 \mathrm{E}-03$ & 0 & $7.82 \mathrm{E}-03$ & 0 & 0 & 0 \\
\hline$" M^{\prime \prime}$ & Control Ignition & $1.39 \mathrm{E}-05$ & $1.39 \mathrm{E}-05$ & 4.17E-04 & 0 & 0 & 0 \\
\hline
\end{tabular}

Results for all Tanks. Runs of the VOI decision model for all tanks provides results that can be combined to provide a profile across all saltcake tanks. First, consider the EVPI. The results for all tanks are shown in Appendix B, and the resulting profile is shown graphically in Figure 4.5. Forty-four of the 65 saltcake tanks had an EVPI of less than $\$ 100,000$; 11 tanks had an EVPI of $\$ 100,000$ to $\$ 250,000$; five tanks had an EVPI of $\$ 250,000$ to $\$ 500,000$; one tank had an EVPI of $\$ 500,000$ to $\$ 1$ million; and four tanks had an EVPI of greater than $\$ 1$ million. The largest EVPI was under $\$ 2$ million.

If sampling and analysis of tank contents costs on the order of at least $\$ 250,000$, then this analysis indicates that it is not worth taking samples from 55 tanks, because even perfect information is not worth the cost of sampling. For the other ten tanks, a more detailed analysis of the value of imperfect information, as described above, would likely indicate that several other tanks should not be sampled because the cost of sampling would exceed the value of the information. 


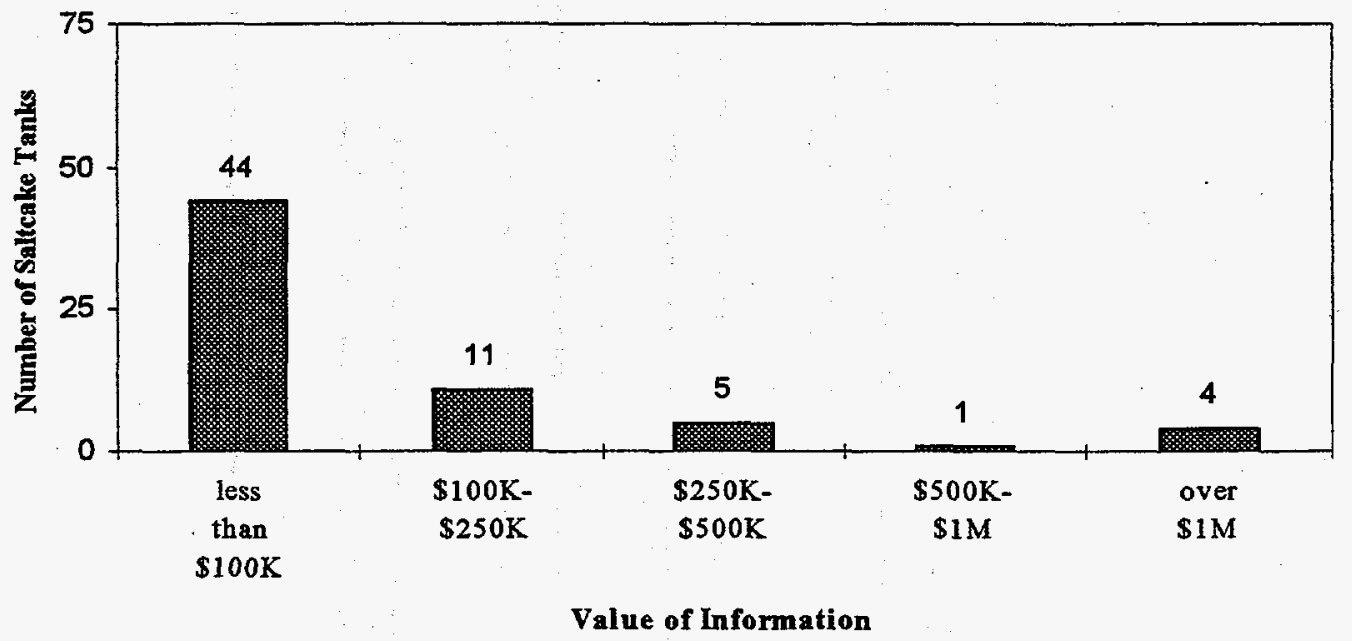

Figure 4.5. Expected Value of Information (EVPI) Profile Condensed Phase Organic-Nitrate Reactions in Saltcake Tanks

For 24 of the 44 tanks, the best decision was to take no further action. The risk was small enough that no further mitigative action was justified. For others, however, mitigation was a better action than collecting more information. As detailed in Appendix B, controlling the ignition sources was a better action to take for the remaining 20 tanks with an EVPI of less than $\$ 100,000$. If no information were sought for the 16 tanks with an EVPI between $\$ 100,000$ and $\$ 500,000$, it would be best to control ignition sources on these tanks as well.

Adding the values of information across tanks provides an approximation to the value of providing information for those tanks. Perfect information about the contents of the five highest-valued tanks was worth approximately $\$ 7$ million. Perfect information about the contents of the ten highest-valued tanks was worth approximately $\$ 9$ million. A similar analysis shows that perfect information about the amount of reactive waste for the four highest-valued tanks was worth approximately $\$ 2$ million. Perfect information about moisture in the four highest-valued tanks was worth approximately $\$ 5$ million.

Resulting Characterization Strategy. Performing the VOI analyses for all tanks and combining the results from all runs provides the basis for formulating a tank characterization strategy for the organicnitrate safety issue. The strategy addresses the following components: what to characterize, where to characterize, how to characterize, when to stop characterizing, and how to use characterization information. 
What to Characterize. The structure of the organic-nitrate VOI decision model shows uncertainty about tank contents represented by the amounts of dry and damp reactive waste. So characterization should be aimed at resolving the TOC and moisture in each tank.

The analysis shows the value of information for each tank. Forty-four tanks have EVPI less than $\$ 100,000$. So based on the results of this analysis alone, the contents of these tanks would not be characterized further. The rest of the tanks are possible candidates for further characterization depending on the cost of gathering information and the quality of information that would be provided. The four tanks with the highest EVPI, each over \$1 million, were TX-105, S-105, TX-106, and TX-102.

For each of the four tanks listed above, the method of characterization should provide information about the moisture in the tank. Information about moisture is more valuable than information about the amount of reactive waste for these tanks. This characterization may be done by auger sample or other methods that provide information about the surface moisture of the waste. Other methods, such as core drilling, that can provide information about both moisture and amount of reactive waste should also be considered. The more expensive methods will need a closer examination of the quality of information that can be provided at different costs, such as might arise through different sample sizes. The VOI of differènt quality also needs to be calculated, as described above.

The EVPI analysis showed that no additional characterization is justified for 44 tanks. More detailed analyses were needed to decide when to stop characterizing the other tanks. Another 16 or 17 may be ruled out for further characterization based on the quality of information that could be provided for under $\$ 500,000$. The remaining four or five needed even more detailed analysis of the quality and cost of information that could be provided by different means to decide on the appropriate characterization method and sample size.

The mitigation strategies show how to use the information provided by characterization to ensure interim safe storage. Twenty-four tanks showed no additional characterization or mitigative action was justified. Twenty to $\mathbf{4 0}$ additional tanks justified no additional characterization but needed ignition sources to be controlled. The remaining tanks that justified additional characterization might also require mitigation, depending on the results of the characterization. (A mitigation was specified as conditional on each possible report of tank contents.)

It is important to note that similar VOI decision analyses would be combined across safety issues and between safety and disposal to provide a comprehensive characterization strategy. Information needs require justification based on a combination of issues, both safety issues and disposal information needs. This example calculation was performed and described for the single safety issue of condensed phase organic-nitrate reactions in saltcake tanks. A more plausible characterization strategy would result from the combination of all safety issues and disposal needs. 


\subsubsection{VOI Analysis for a Flammable Gas Tank}

This section describes the application of the recommended strategy for a safety issue relevant to Tank 241-S-106, a HLW storage tank at Hanford. The flammable gas safety issue was selected because it is an issue that potentially exists in many of the HLW tanks at Hanford. This example uses currently available information and shows how implementing the strategy integrates the technical basis for safety issue closure, probabilistic risk calculations, cost of alternative actions, and decision analysis methods, thereby providing the basis for decisions, including justification for characterization, in a risk-based context. The scope of the example has been selected so that all aspects of the strategy implementation can be illustrated in an integrated manner. This section summarizes the analysis of the example; the flammable gas safety issue is also discussed.

\subsubsection{Flammable Gas Safety Issue Description}

Flammable gases, primarily hydrogen, are generated in HLW at Hanford by both radiolytic and chemical processes. A synergistic relationship between these processes is demonstrated for some wastes (Bryan and Pederson 1996). Gaseous reaction products other than hydrogen include nitrogen, nitrous oxide, ammonia, methane, oxygen, and oxides of carbon. The exact composition of the gases produced and the quantity produced as a function of time depend on the waste composition and temperature. A mixture of gases may be generated that is flammable without the need for first mixing in air to be within their flammability range (for example, hydrogen and nitrous oxide have a flammable mixture range). However, prediction of the flammability range of these potential gas mixtures is difficult, and little directly measured information exists.

Normally, the gases produced are expected to migrate through the waste at about the same rate as they are produced (the gases generally have low solubility in the aqueous phase, ammonia being the notable exception, and transport through the solid phases occurs by permeation). Once released from the waste, the gases enter the tank dome space where they are subject to dilution and transport by normal gas phase processes. Some tanks employ active forced ventilation of the tank dome space while others rely on static, or passive, ventilation. Thus, the rate at which released gases are diluted and mixed with the atmosphere within the tank dome space and subsequently released to the external atmosphere depends on individual tank characteristics (e.g., volume of the tank dome space, temperature, active or passive ventilation, ventilation system characteristics, quantity of released gas, and rate of gas release). Measurement of flammable gas concentrations in the tank dome space indicate a very low flammable gas concentration in the dome space of many tanks (Hodgson 1996). This condition could exist at the time of the measurement if 1) the gases are not retained but are continuously released and mixed within the tank dome space; 2) the gases generated were retained within the waste and were released at a rate about equal to their production to the external atmosphere; 3) the gases generated were retained in the waste and were not released into the tank dome space in a time period that would result in their detection at the time of the measurement; or 4) the quantity of flammable gas generated and released from the waste is small enough that the released gas is thoroughly mixed with and diluted by the tank atmosphere at the point of measurement. 
Some tanks containing both fluid and settled solids retain gas in the settled solids layers. These gases may possibly be released suddenly by a disturbance such as core sampling (LANL 1996), saltwell pumping (WHC 1996), seismic events, ${ }^{(a)}$ or retrieval. A few tanks, such as 241-SY-101, exhibit episodic spontaneous gas releases or "burps" requiring no external disturbance. Gas released episodically from the waste into the dome space may remain flammable for some time (minutes to days) (Babad et al. 1992; Allemann et al. 1991). Tank 241-SY-101 is thus far the worst known case for such episodic gas releases at Hanford (the episodic gas releases in this tank have been mitigated by the installation of a mixer pump that is used periodically to stir the gas-retaining solids phase, thereby releasing the retained gas more frequently and in small volumes).

If a sufficient volume of gas were released and ignited while it remained flammable, the subsequent burn could produce pressures high enough to cause failure of the tank ventilation system, including the HEPA filters, the tank structure, and/or the associated tank components. An event of this kind could lead to a release of waste to the environment. Even relatively small volumes of flammable gases can be a safety issue if the gases collect in pockets that are exposed to ignition sources. A flammable gas burn within the waste itself is also considered possible though very unlikely.

The implementation of the risk-based characterization strategy for this example is described below. The desired outcome for the example is defined as the risk associated with the flammable gas safety issue being within acceptable levels. As pointed out in Section 2.3, definition of the acceptable level of risk is a DOE responsibility. The specific requirements considered in this example are given in Section 4.1.2.2 and summarized in Table 4.5. This desired outcome may be achieved by 1) resolution through knowledge that existing risks are acceptable, 2) mitigation to change waste or safety issue-related conditions sufficiently to make the risks acceptable, or 3) implementing controls to properly manage tank operational aspects related to safety issues for which risks are not known to be acceptable.

\subsubsection{Results and Conclusions}

The example shows that it is possible to make technically sound and defensible decisions based on current information, even if the current information is minimal. This section will summarize the conclusions drawn from analysis of the example.

Risk-Based Acceptance Criteria and Decisions. A technically sound and defensible set of decisions using current information based on acceptable risk is the foundation of the strategy. It is necessary, however, to have definite and unambiguous criteria for making the decision. The following cases illustrate how decisions will be made under different criteria.

(a) Reid HR, PA Meyer, and J Phillips. "Waste Tank Sludge Yielding Estimates with Design Base Earthquake Spectrum Shock Analysis." Presentation at the Flammable Gas Quarterly Review Meeting, May 1996. Pacific Northwest National Laboratory, Richland, Washington. 
The decision to be made will be decision A, B, or C. Decision A is resolution through understanding, where the risk due to a flammable gas burn in Tank 241-S-106 is known to be acceptable. Decision B is acceptable risk through mitigation or controls, where an active ventilation system would be installed in Tank 241-S-106 to mitigate the chance of flammable gas burn. Decision C is to gather information, where more information concerning the waste and tank conditions in Tank 241-S-106 or about mitigation options would be obtained before selecting an action.

The above set of options is a type of triage, where either decision A or decision B is chosen when the recommendation based on current information is unambiguous, and indefinite cases are passed on to the next stage of analysis, which involves a detailed VOI analysis. The results of the more detailed VOI analysis determine whether additional characterization is warranted. The decision under investigation in this example is considered under several different criteria and interpretations of criteria, each having been employed at some time on the Hanford Site. The criteria and their interpretations are listed in Table 4.5.

Based upon the analysis described in the following sections, the triage decision for these options is as follows:

Table 4.5. Risk Acceptance Criteria under Consideration

\begin{tabular}{|c|l|l||}
\hline Criterion & \multicolumn{1}{|c|}{ Definition } & \multicolumn{1}{|c|}{ Interpretation } \\
\hline \hline I & No potential for flammable gas burn & $\begin{array}{l}\text { The likelihood (or chance) for having even a } \\
\text { small flammable gas burn is zero. }\end{array}$ \\
\hline II & $\begin{array}{l}\text { The frequency of flammable gas burn is } \\
\text { less than } 10^{-6} \text { per year }\end{array}$ & $\begin{array}{l}\text { Flammable gas burns (including those that } \\
\text { cause no damage) may occur at an expected } \\
\text { rate of no more often than } 10^{-6} \text { times in a year } \\
\text { (once in a million years). }\end{array}$ \\
\hline III.a & $\begin{array}{l}\text { The frequency of dome failure resulting } \\
\text { from a flammable gas burn is less than } \\
10^{-6} \text { per year, as defined by the expected } \\
\text { frequency of dome failure less than } 10^{-6} \\
\text { per year }\end{array}$ & $\begin{array}{l}\text { A tank dome failure as a result of a flammable } \\
\text { gas burn may occur no more often than } 10^{-6} \\
\text { times in a year (once in a million years), as } \\
\text { defined by the expected or mean frequency } \\
\text { less than 10-6 times in a year. }\end{array}$ \\
\hline III.b & $\begin{array}{l}\text { The frequency of dome failure resulting } \\
\text { from a flammable gas burn is less than } \\
10^{-6} \text { per year, as defined by the } 95^{\mathrm{t}} \text { per- } \\
\text { centile of frequency of dome failure less } \\
\text { than } 10^{-6} \text { per year. }\end{array}$ & $\begin{array}{l}\text { A tank dome failure as a result of a flammable } \\
\text { gas burn may occur at an expected rate of no } \\
\text { more often than 10 } 10^{-6} \text { times in a year (once in a } \\
\text { million years), as defined by the 95\% } \\
\text { confidence level of the predicted frequency } \\
\text { being less than } 10^{-6} \text { times in a year. }\end{array}$ \\
\hline IV & $\begin{array}{l}\text { Offsite and onsite dose is acceptable } \\
\text { The dose consequence from a flammable gas } \\
\text { burn is at an acceptable level. }\end{array}$ \\
\hline
\end{tabular}


Criterion I: If risk acceptance criterion I is imposed, the recommendation is decision C, gather information. Since the analysis shows more than a zero chance for a flammable gas burn in Tank 241-S-106, the risk acceptance criterion is not met under current conditions based on the existing information. Consequently, the resolution decision (decision A) cannot be made. Similarly, installing an active ventilation system will not remove the potential or chance for flammable gas burn as long as the waste produces flammable gas. Consequently, mitigation decision (decision B) will not meet the risk acceptance criterion. The risk is not acceptable, and other mitigation options must be considered and/or new information must be obtained that will remove the risk in order to meet criterion I. Except for retrieval of the tank contents or other modification of the waste contents as a mitigation action, it is unlikely that this acceptance criterion can be met. There is a remote chance that the gas produced by the waste is not flammable; thus a remote chance that pursuit of decision $C$ would result in a determination that the gas produced by the waste is not flammable. A better decision might be to recognize that this is an unrealistic criterion to apply to many (most) tanks.

Criterion II: If risk acceptance criterion II is imposed, the recommendation is decision C, gather information. The frequency of a flammable gas burn of any size is not shown by this analysis to be less than $10^{-6}$. Therefore, resolution (decision A) cannot be asserted. Since active ventilation does not affect the frequency of burns from small volumes of flammable gas, the proposed mitigation action (decision B) is not effective. Additional information and analysis (decision $\mathrm{C}$ ) would focus on a more detailed analysis of the contributing factors to the risk, especially with regard to the frequency of plume burns of small volume. Strict controls on ignition sources might lead to an acceptable risk under this criterion.

Criterion III.a: If risk acceptance criterion III.a is imposed, the recommendation is decision A, resolution through understanding. The analysis shows that the expected frequency of dome failure due to a flammable gas burn is calculated in this example to be $8 \times 10^{-7}$. According to this criterion, the risk from flammable gas is acceptable. No mitigation is necessary (decision B), nor is any further information gathering and analysis warranted (decision $C$ ). The expected life-time cost incurred by this decision is $\$ 1300$, based on a per-accident estimate of $\$ 500 \mathrm{M}$.

Criterion III.b: If risk acceptance criterion III.b is imposed, the recommendation is decision C if there are several tanks of this type to which this new information would be valuable, or decision $B$ otherwise. The $95^{\text {th }}$ percentile value for the frequency of dome failure due to a flammable gas burn is $3 \times 10^{-6}$ (see Figure 4.6). The large difference between this value and the expected value of the frequency $\left(8 \times 10^{-7}\right)$ is due to the large uncertainty in the information about this tank. This value must be reduced by a factor of three to produce an acceptable risk; therefore, resolution is not demonstrated (decision A). The factor of three reduction is easily obtainable by the mitigation action of active ventilation (decision B). However, it may also be possible to ascertain from new information (decision $\mathrm{C}$ ) that the risk is actually less than the calculated $95^{\text {th }}$ percentile risk value. The uncertainty in the risk is sensitive to the uncertainty in/about passive ventilation rates, retained gas composition, and spark frequency, and there are existing, relatively low-cost options for obtaining this information. The sensitivity analysis shows that it will be necessary to obtain new information on more than one of these parameters to achieve the required factor of three reduction in the uncertainty of the risk. While it is not possible to obtain new information on several of these 


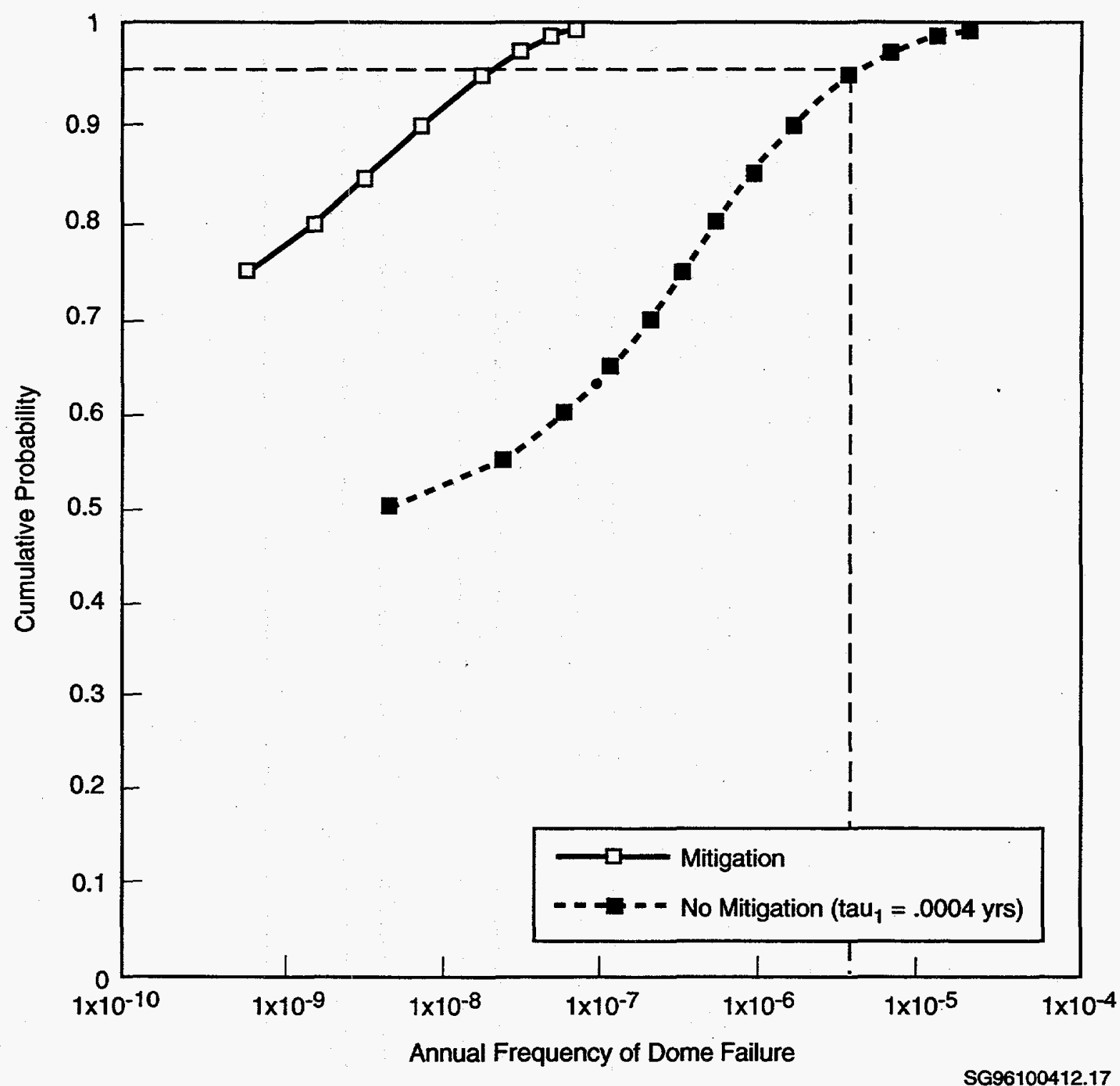

Figure 4.6. Annual Frequency of Dome Failure (value on horizontal axis is $3 \times 10^{-6}$ for annual frequency of dome failure of the point whose cumulative probability [on the vertical axis] is $\mathbf{0 . 9 5}$ 
parameters for less cost than the mitigation option $(\$ 700 \mathrm{~K})$ for a single tank, a possible cost-effective option is to perform such studies on a few related tanks in the Hanford S-Farm and then apply the results to reduce the uncertainty in the estimated risk in many of the S-Farm tanks. Based on this example, it can be recommended that this VOI analysis be performed before a decision is made to install active ventilation in Tank 241-S-106.

Note that the VOI analysis may still show that active ventilation should be installed (decision B). The advantage of performing the analysis is that the cost benefit, as well as the benefit from risk reduction, will be known and documented to aid in defending this decision if it is challenged.

Tank failure from a seismic event is predicted to occur with a frequency of at least 1 in 7500 years $\left(1.3 \times 10^{-4}\right.$ per year) by some estimates (LANL 1996). Therefore, the acceptable level of $10^{-6}$ annual frequency for tank failure from a flammable gas burn is somewhat arbitrary, and it might be more costeffective to focus efforts and budgets on reducing the risk from failure of single-shell tanks due to seismic events. This could be accomplished by accelerated salt-well pumping and transferring the waste into double-shell tanks. Likewise, the expected (and in some cases demonstrated) loss of tank integrity due to corrosion and the plans to remove liquid wastes from single-shell tanks places a time scale on the problem that should be considered in deciding on what level of risk is acceptable. If this tank is to be salt-well pumped within a few years, is a risk of $3 \times 10^{-6}$ may be acceptable. This illustrates how the application of the strategy provides options with quantifiable risks to decision-makers but does not prescribe solutions.

Criterion IV: If risk acceptance criterion IV is imposed, the recommendation is decision A, resolution. The expected offsite and onsite doses from a dome failure accident were calculated to be essentially zero offsite, and onsite doses were less than 100 mrem for 10 workers. According to any historical version of Hanford risk-acceptance guidelines, this dose is acceptable, even at an annual frequency of one per year. A dome failure accident is obviously not acceptable at a frequency of one per year. Therefore, it is probable that this criterion alone would be insufficient for making defensible decisions. However, it is valuable for the decision maker to be able to point out that the expected dose from a dome failure is well within guidelines to assure stakeholders that their concerns are being addressed.

The recommended decisions that apply to each of the risk acceptance criteria discussed in this section are summarized in Table 4.6. Each decision that recommends gathering information, provides the most insight in addressing the ultimate resolution or mitigation decisions.

\subsubsection{Comparison to Traditional Bounding Calculations}

A typical conservative or "bounding" calculation takes a conservative estimate for each parameter value and uses these point values in the deterministic risk model. This procedure is a valid approach to obtaining an upper bound on the risk; however, the results may often be overly conservative when compared with the actual risk. Using the $95^{\text {th }}$ percentile values for each of the parameters in the calculation of risk (Equation 4.1) provides a highly conservative estimate for the probability of dome failure as $\mathbf{0 . 0 1 4}$ 
Table 4.6. Summary of Decisions for Flammable Gas Burn in 241-S-106

\begin{tabular}{|l|l|l||}
\hline Risk Acceptance Criterion & \multicolumn{1}{|c|}{ Decision } & \multicolumn{1}{c|}{ Parameter } \\
\hline I & Gather information & $\begin{array}{l}\text { - Equivalent hydrogen fraction } \\
\text { - Retained gas volume } \\
\text { - Time at risk }\end{array}$ \\
\hline II & & $\begin{array}{l}\text { - Spark frequency } \\
\text { - Retained gas volume }\end{array}$ \\
\hline III.a & Gather information & - Not applicable \\
\hline III.b & Resolution & $\begin{array}{l}\text { - Characteristic ventilation time } \\
\text { - Spark frequency } \\
\text { - Retained gas volume }\end{array}$ \\
\hline IV & $\begin{array}{l}\text { Gather information (or miti- } \\
\text { gate depending upon VOI } \\
\text { analysis) }\end{array}$ & - Not applicable \\
\hline
\end{tabular}

(1.4\%). Alternatively, the $95^{\text {th }}$ percentile value for the probability of dome failure may be read directly from Figure 4.7 as $2 \times 10^{-5}$. This value is three orders of magnitude smaller than the highly conservative, bounding risk calculation. This shows the bounding calculation is in fact bounding, but it is more conservative than is warranted.

The primary reason for the difference is that each of the conservative parameter values is somewhat unlikely and is statistically independent. The chance of achieving all conservative parameter values simultaneously is extremely unlikely; thus the highly conservative, bounding risk calculation is extremely improbable. The actual (direct) $95^{\text {th }}$ percentile of the risk is, generally speaking, an accumulation of a number of somewhat unlikely combinations of conservative and nominal parameter values. The probability of dome failure in these cases is, therefore, not as large as the highly conservative calculation.

Although a bounding analysis of the consequence was not calculated, experience in the organic nitrate safety program has shown that using conservative source terms such as the "supertank" source also leads to overly conservative bounds of the consequence (Webb et al. 1995). The supertank source term represents the highest composition ever documented for every constituent under consideration and applying this source term to any and every tank.

Decisions based on overly conservative bounds consume resources by encouraging unjustified characterization and unnecessary mitigation activities. 


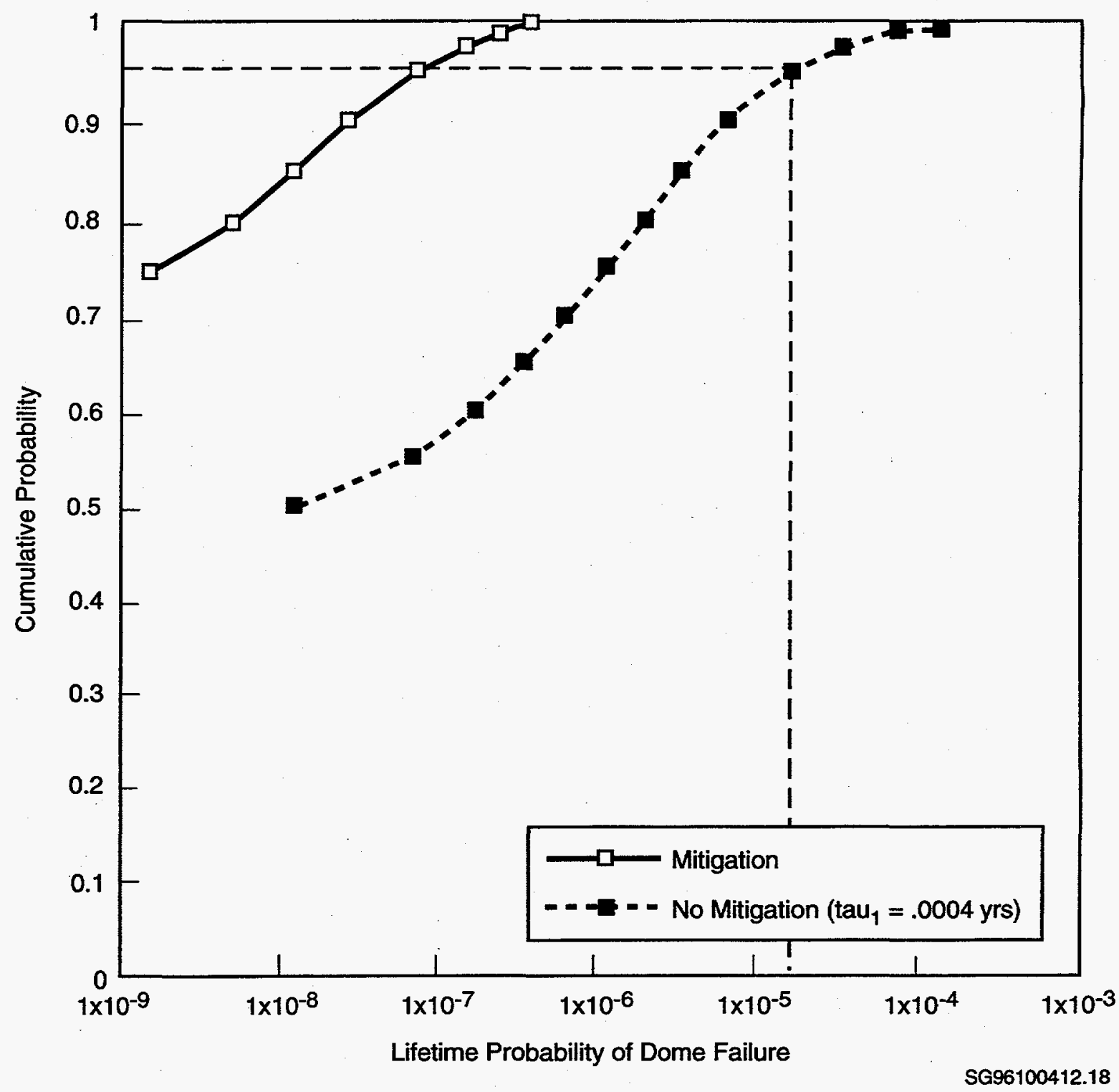

Figure 4.7. Cumulative Distribution of Lifetime Dome Failure Probability. The value on the horizontal axis is $2 \times 10^{-5}$ for the lifetime probability of dome failure for the point whose cumulative probability (on the vertical axis) is $\mathbf{0 . 9 5}$. 


\subsubsection{Scope of Example Application of the Recommended Strategy}

Tank 241-S-106, a single-shell tank, was selected as the example for examining the utility of the strategy. This waste tank is currently not on the flammable gas watch list, ${ }^{(2)}$ but based on current concepts (Brewster et al. 1995), the behavior of the waste suggests a potential for generation, retention, and for episodic release of gases. The waste behavior that indicates this tank may be flammable gas safety concern are 1) a rise of about $50 \mathrm{~cm}$ in waste surface level since the date of last waste level adjustment (June 1982) and 2) the change in waste surface level as a function of barometric pressure is one of the largest observed to date for a single-shell tank at Hanford (Hopkins 1995). ${ }^{\left({ }^{(b)}\right.}$ Without relevant measurements, it is unknown whether the gases generated and released are flammable or if the waste properties allow for episodic releases of significant volumes of the trapped gases.

This tank contains waste that is predominantly of the salt cake type (Agnew 1996; Brevick 1995d). The waste in this tank has not been core sampled and analyzed, nor has the tank dome space been sampled and analyzed. The tank is passively ventilated. A tank characterization report for this tank has not been issued; the tank has not been interim stabilized (i.e., it has not been salt-well pumped to remove as much free liquid as feasible). Thus, direct information regarding the tank contents and the composition of the gases in the tank dome space does not presently exist (Hanlon 1996). Implementation of the recommended strategy for our limited scope example using Tank 241-S-106 is therefore typical of applying the strategy to many of the existing waste tanks and their associated potential safety issues.

The strategy employs a structured logic approach for technical problem analysis; the structured logic diagram applicable to Tank 241-S-106 is shown in Figure 4.8. This diagram is consistent with, but not identical to, the comprehensive structured logic diagram for the flammable gas safety issue. The symbols and abbreviations used in Figure 4.8 are shown in Figure 4.9. A detailed description of the flammable gas structured logic diagram, including its development, is in Stewart et al. (1996).

Optimal management of the risk of a flammable gas burn in a tank dome space requires making decisions regarding characterization (gaining new, additional chemical and physical intelligence), reducing uncertainties in information, models, mitigation options, operational controls, and the costs associated with each.

This example considers a release of flammable gas from the waste into the tank dome space followed by its ignition by an uncorrelated ignition source. An uncorrelated ignition source is one in which the probability of occurrence is statistically independent of the probability of a gas release. The example does not consider a burn of a plume of flammable gas as it is released from a specific location (area) in the tank waste surface (typically called a plume burn).

(a) A watch list tank is one that has been identified in accordance with Public Law 101-501, Section 3137, "Safety Measures for Waste Tanks at Hanford Nuclear Reservation," 1990. Such tanks have operating specifications that have been adopted to prevent injury to personnel or damage to the facility or environment that may be more stringent than those applied to waste tanks not on a watch list.

(b) Brewster ME and BJ Palmer. 1995. Prioritization of Single-Shell Tanks Study of Gas Retention and Episodic Release. PNL-WTS122295, Pacific Northwest Laboratory, Richland, Washington. 


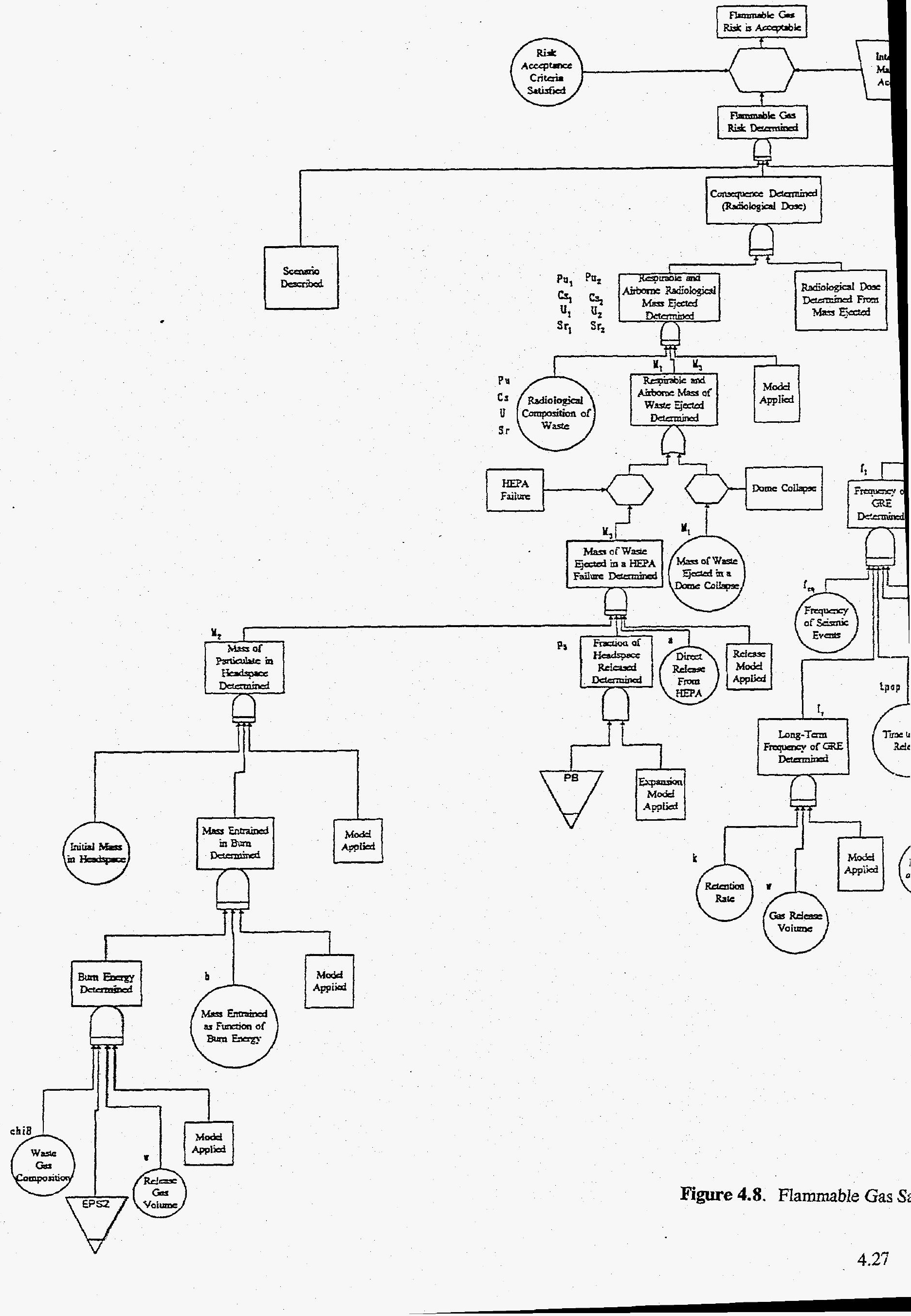




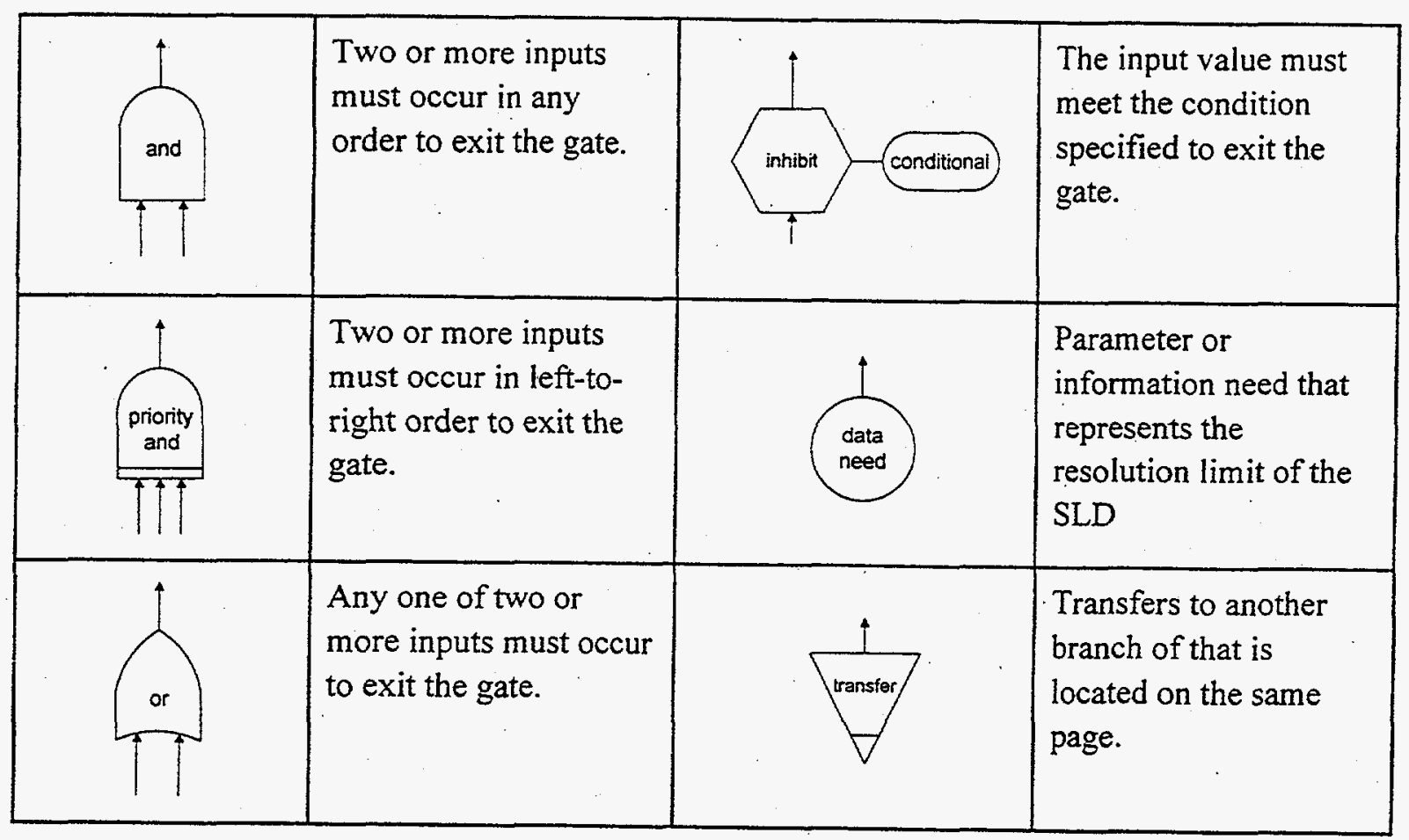

Figure 4.9. Structured Logic Diagram Symbols

A flammable gas burn in the tank dome space is expected to result in an increase in pressure inside the tank dome space. This pressure increase could 1) lead to failure of the tank ventilation system including the HEPA filters, 2) cause structural damage to the tank, and 3) possibly result in tank dome failure into the waste with resulting tank waste released to the environment.

The example uses a tank dome failure as an illustrative event. Mitigation of the event is considered by installation of permanent active ventilation of the tank dome space. This option was identified through use of the structured logic diagram, calculations of probabilities of the event, and sensitivity analysis of the calculation. Since increased ventilation is a standard mitigative action for flammable gas hazards in industry, technical and cost information were readily available for this mitigation option.

The example also considers obtaining additional (justified) information regarding the composition of the gas, its flammability, the energy expected to be released in a burn, and the potential volume of gas that might be released. The results of this analysis also provide insight into whether obtaining (new) information pertinent to the tank waste is justified and cost-effective.

Controls to reduce the risk are not explicitly analyzed in this example. However, the estimates of frequency of the assumed ignition source (spark) are based on certain controls being in place.

More permanent solutions to the flammable gas safety issue, such as retrieval and processing of the waste, were not considered in this example. Salt-well pumping of the tank is also expected to mitigate the flammable gas safety issue for this specific tank because of the predicted saltcake nature of the waste. 


\subsubsection{Risk Analysis}

The risk associated with an event, Risk $\mathrm{e}_{\mathrm{i}}$ is the product of the probability (Probability) ef $_{\mathrm{E}}$ the event occurring and the consequences (Consequence) ${ }_{\mathrm{E}}$ resulting from its occurrence:

$$
\text { Risk }_{\mathrm{E}}=\left(\text { Probability }_{\mathrm{E}} \text { (Consequence) }\right)_{\mathrm{E}}
$$

The consequence resulting from a tank dome failure is essentially uncoupled from the calculation of the probability of the dome failure. Neither mitigation or characterization options affect the calculation of consequences. Only the probability calculation is decomposed and discussed in detail in this chapter. To simplify this example, synergistic effects were not considered (i.e., other disruptive events, such as an organic waste fire that might initiate [or be caused by] a flammable gas fire, were not included in the analysis for either probability or consequence calculations).

\subsubsection{Calculation of Probability}

The structured logic diagram in Figure 4.8 is the basis for a mathematical model used to calculate the probability of a tank dome failure. Tables 4.7 and 4.8 describe the input parameters and the step-by-step deterministic calculation of probability of dome failure, $\mathrm{p}_{4}$. Each input parameter and intermediate calculation corresponds to an item in the structured logic diagram. The input parameters and intermediate calculations are defined in the tables. Simple but defensible physical and empirical models were used to provide close-form expression for each intermediate calculation. Equation 4.3 (the mathematical equation to deterministically calculate the probability of tank dome failure, based on Figure 4.8) is shown to illustrate the form of the solution obtained from this somewhat simplified model.

$$
\begin{aligned}
& \left(0 \quad \text { if } \frac{\tau_{1} \chi_{F} R F V_{r e t}}{t_{r} V_{\text {dome }} L F L}\left(1-\exp \left(-\frac{t_{r}}{\tau_{1}}\right)\right) \leq 1,\right. \\
& p_{4}=\left\{1-\exp \left(-f_{s} f_{e q}\left(t_{r}-\tau_{1} \ln \left(\frac{\tau_{1} \chi_{F} R F V_{\text {ret }}}{\tau_{1} \chi_{F} R F V_{\text {ret }}-t_{r} V_{\text {dome }} L F L}\right) \quad\right. \text { otherwise. }\right.\right.
\end{aligned}
$$

where $\mathrm{p}_{4}$ is the probability determined by carrying the calculation through the structured logic diagram.

Very few of the tank-specific data needed for the probability calculations exist for the example tank. In the absence of tank-specific information, a regulatory-accepted formal process was used to obtain the needed expert judgements for all necessary parameter values and distributions used in the calculations. The procedure was adapted from a methodology developed for the Nuclear Regulatory Commission (NRC) as 
Table 4.7. Input Parameters

\begin{tabular}{|c|c|c|c|}
\hline Name & Symbol & Nominal Value & Description \\
\hline Time at risk & $\mathbf{t}$ & 3 yrs & $\begin{array}{l}\text { The time at risk for a flammable gas burn (i.e., from now until } \\
\text { resolution of the flammable gas safety issue for the tank in } \\
\text { question via salt-well pumping). }\end{array}$ \\
\hline Spark frequency & $f_{\mathbf{s}}$ & $1 \mathrm{E}-2$ per yr & $\begin{array}{l}\text { The expected number of sparks that could initiate a flammable gas } \\
\text { burn per year. }\end{array}$ \\
\hline Gas release frequency & $\mathbf{f}_{\mathrm{eq}}$ & 1E-3 per yr & $\begin{array}{l}\text { The rate at which gas release events (GREs) of sufficient magni- } \\
\text { tude to lead to dome failure if ignited occur in the tanks in } \\
\text { question. }\end{array}$ \\
\hline Duration of release & $\mathbf{t}_{\mathbf{r}}$ & $5 \mathrm{E}-4$ yrs (4 hrs) & $\begin{array}{l}\text { The length of time that gas is released from the waste in a single } \\
\text { GRE. The release rate is assumed constant over this period. }\end{array}$ \\
\hline Characteristic ventilation time & $\operatorname{tau}_{1}$ & $3 \mathrm{E}-2$ yrs (10 days) & $\begin{array}{l}\text { The time necessary for ventilation (passive or active in the case of } \\
\text { mitigation) to remove a volume equal to the headspace volume. }\end{array}$ \\
\hline Headspace volume & $\mathrm{V}_{\text {dome }}$ & $2200 \mathrm{~m}^{3}$ & $\begin{array}{l}\text { The volume inside the tank that is not occupied by solid or liquid } \\
\text { waste. }\end{array}$ \\
\hline Retained gas volume (STP) & $\mathrm{V}_{\mathrm{ret}}$ & $550 \mathrm{~m}^{3}$ & $\begin{array}{l}\text { The amount of gas retained in the waste at the time a release } \\
\text { occurs. }\end{array}$ \\
\hline Release fraction & RF & 0.5 & The fraction of the retained gas that is released to the headspace. \\
\hline Equivalent hydrogen fraction (flammability) & $\mathrm{chi}_{p}$ & 0.4 & $\begin{array}{l}\text { A description of the flammability of the actual waste gas relative } \\
\text { to pure hydrogen. }\end{array}$ \\
\hline Lower flammability limit of hydrogen in air & LFL & 0.04 & $\begin{array}{l}\text { The smallest concentration (molar) of hydrogen in air that will } \\
\text { sustain combustion. }\end{array}$ \\
\hline Pressure at which gas is held in the waste & $\mathbf{P}$ & $2 \mathrm{~atm}$. & $\begin{array}{l}\text { Pressure due to hydrostatic forces at which retained gas is held in } \\
\text { the waste. }\end{array}$ \\
\hline
\end{tabular}


Table 4.8. Intermediate Calculations ${ }^{(a)}$

\begin{tabular}{|c|c|c|c|}
\hline Name & Symbol & Formula & Description \\
\hline Probability of dome failure & $p_{4}$ & $1-\exp \left(-\mathrm{f}_{c} * t\right)$ & The probability of at least one dome failure occurring. \\
\hline Frequency of dome failure & $f_{c}$ & $\mathbf{f}_{\mathrm{c}}=\mathbf{f}_{\mathrm{b}}^{*} \mathbf{p}_{1}$ & $\begin{array}{l}\text { The annual frequency of dome failure is the probability of } \\
\text { dome failure in one year. }\end{array}$ \\
\hline $\begin{array}{l}\text { Fraction of time headspace is } \\
\text { flammable }\end{array}$ & $\mathbf{p}_{\mathbf{1}}$ & $\mathrm{p}_{1}=\mathbf{f}_{\mathrm{s}} * \operatorname{tau}$ & The probability of the headspace being flammable. \\
\hline Duration of hazard per GRE & tau & $\operatorname{tau}=t_{a}+t_{b}$ & $\begin{array}{l}\text { The duration following a given GRE of conditions that could } \\
\text { produce a flammable gas burn of sufficient energy to cause a } \\
\text { dome failure. The presence of a flammable gas composition is } \\
\text { necessary for a hazardous condition. }\end{array}$ \\
\hline Duration of hazard during release & $t_{\mathrm{a}}$ & $\begin{array}{c}\mathbf{t}_{\mathrm{a}} / \mathrm{t}_{\mathrm{r}}=1-\left(1 / \mathrm{eps}_{1}\right) * \\
\ln \left(\operatorname{lambda} \mathrm{a}_{1} /\left(\operatorname{lambda}_{1}-1\right)\right)\end{array}$ & $\begin{array}{l}\text { That portion of the duration of hazard (tau) that occurs while } \\
\text { gas is being released from the waste. }\end{array}$ \\
\hline Duration of hazard after release & $t_{b}$ & $t_{b} / t a u_{1}=\ln \left(\operatorname{lambda}_{f}\right)$ & $\begin{array}{l}\text { That portion of the duration of hazard (tau) that occurs after } \\
\text { gas is released from the waste. }\end{array}$ \\
\hline Threshold condition & lambda & $\begin{array}{l}\text { lambda }=1 \operatorname{lambda}_{1} * \\
\quad\left(1-\exp \left(-\mathrm{eps}_{1}\right)\right)\end{array}$ & $\begin{array}{l}\text { The maximum fraction of the lower flammability limit (LFL) } \\
\text { achieved from a given gas release. If lambda }<1 \text {, then the } \\
\text { duration of hazard (tau) is zero. Otherwise the given formulas } \\
\text { for } t_{a} \text { and } t_{b} \text { apply. }\end{array}$ \\
\hline $\begin{array}{l}\text { Fraction of LFL achieved at steady } \\
\text { state }\end{array}$ & lambda & $\begin{array}{c}\text { lambda }{ }_{1}=\left(\operatorname{tau}_{1} * \mathrm{chi}_{\mathrm{p}} * \mathrm{RF}^{*} \mathrm{~V}_{\mathrm{ret}}\right) / \\
\left(\mathrm{t}_{\mathrm{r}} * \mathrm{~V}_{\text {dome }^{*}} * \mathrm{LFL}\right)\end{array}$ & \\
\hline $\begin{array}{l}\text { Ratio of duration of release to } \\
\text { characteristic ventilation time }\end{array}$ & eps & $\mathrm{eps}_{1}=\mathbf{t} / \mathrm{tau}_{1}$ & $\begin{array}{l}\text { This is a dimensionless group measuring the duration of the } \\
\text { gas release relative to the characteristic ventilation time. If } \\
\text { this quantity is much less than } 1 \text {, the gas release is considered } \\
\text { "fast." }\end{array}$ \\
\hline
\end{tabular}


part of the NUREG-1150 risk analysis of several nuclear power plants (NRC 1989; Keeney and von Winterfeldt 1991). Staff members from Pacific Northwest National Laboratory, Westinghouse Hanford Company, and Los Alamos National Laboratory, who are knowledgeable about the specific parameters to be elicited, were identified. Others from these organizations also were selected to critique the mathematical model and spreadsheet calculations. The elicitation was led by nationally recognized experts on obtaining and using expert judgements for complex technical problems. It is important to note that expert judgment can be used effectively and defensibly in the absence of definitive, specific information regarding the tank wastes to start the process. The results obtained using such information can then be evaluated to determine whether certain model parameters need better definition; if so, the optimum way to obtain the improved parameters can be determined. Specific information on waste properties, tank dome space gas composition, tank system information such as ventilation rates and structural information, experimental data from waste simulants or waste samples, and improvements in models may be required to reduce the uncertainties in the relevant variables and parameters and the calculated risk.

The nominal values for the input parameter obtained from the elicitation process are given in Table 4.7. The model containing the intermediate calculations was also validated through the elicitation process. The input parameters were entered and the calculations performed using an Excel $^{\mathrm{TM}}$ spreadsheet program.

Values for the dome failure probability are not excessive. An estimate of the value of obtaining additional information that perhaps would reduce the uncertainty in a parameter and the overall risk is justified for certain criteria as summarized in Table 4.6.

\subsubsection{Calculation of Consequences}

If tank dome failure occurs, some quantity of the tank waste is expected to be released into the environment. The quantity predicted to be released was obtained by the elicitation process, as were the specific composition of the waste, its particle size range, and fraction in each size range. For this example, only four elements were used ( $\mathrm{Pu}, \mathrm{Cs}, \mathrm{U}$, and $\mathrm{Sr}$ ); the most toxic isotope for each radionuclide was used for dose and risk calculations.

The total risk of the flammable gas safety issue includes contributions from many sources including health and safety risks to workers and the public, cleanup costs if tank contents are released into the environment, programmatic costs in both monies and credibility, and impact on other Hanford operations.

The predicted quantity of waste released and its predicted composition and particle size range result in the risk measure contours not reaching the Hanford Site boundary; therefore, many of the costs for the risk measures considered are zero. If the amount of material released were a hundred times larger, the contours would extend offsite, and costs for those measures dependent on the size and location of the contaminated area would increase greatly to a value proportional to the size and location of the area affected. 
For this test case, the costs of the adverse consequences of the dome failure scenario (in-tank flammable gas burn, dome failure, and release of $10 \mathrm{~kg}$ of material in the respirable particle size fraction that contributes to radiation dose of an exposed individual) is about $\$ 500 \mathrm{M}$, with the overwhelming cost impact resulting from impacts on other activities at Hanford..$^{(a)}$

\subsubsection{Decision Analysis}

The risk analysis is combined with mitigation options to present a structured decision analysis that will enable investigators to determine the appropriate plan of action for Tank 241-S-106 for the purposes of addressing the issues surrounding flammable gas safety. The influence of each parameter on the risk of a flammable gas burn as measured by the dome failure event is taken into account. Uncertainty in the parameter measurements and the ability to reduce the uncertainty are a significant consideration in justifying additional information collection and analysis.

\subsubsection{Decisions Applicable to Flammable Gas Safety}

The decision to be made is resolution, mitigation, or to gather information. The decision is based on the risk of flammable gas burn for Tank 241-S-106, the risk acceptance criteria that must be met, and the expected costs for each of the decisions. The risk of flammable gas burn for Tank 241-S-106 is estimated based on current knowledge about tank parameters that contribute to the conditions that must exist for a flammable gas burn to occur, including the knowledge of the level of uncertainty surrounding the values of those parameters and the consequences that would result if a flammable gas burn did occur.

The input parameters identified by the risk analysis are characteristic ventilation time, spark frequency, equivalent hydrogen fraction, retained gas volume, and time at risk. The straightforward decision analysis for Tank 241-S-106 shows that the expected lifetime value for frequency of dome failure due to a flammable gas burn is $8 \times 10^{-7}$. The expected cost is $\$ 1,300$ for making the decision that the safety issue is resolved based on a per accident cost of $\$ 500 \mathrm{M}$, compared with the mitigation cost of $\$ 700 \mathrm{~K}$ and the cost of gathering information (dependent on specified parameters for gathering information). Sensitivity analysis is performed to ascertain the parameters that have the most effect on the uncertainty risk. This is done by varying the parameters individually and showing the range of the risk calculation result. In Figure 4.10 , the five flammable gas burn parameters have been varied between their $5^{\text {th }}$ and $95^{\text {th }}$ percentile values and are shown versus the tank lifetime probability of dome failure. Changes in characteristic ventilation time have the most impact on changing the probability of dome failure, thus providing insight into the parameter that should be manipulated to help reduce risk for a flammable gas burn.

The risk analysis calculation in combination with a decision analysis software product, DPL ${ }^{\mathrm{TM}}$, was used to easily perform sensitivity analyses based on the uncertainties of the input parameters. The sensitivity analysis identified the input parameters having the greatest influence on the uncertainty of the

(a) The quantity of waste ejected into the environment and its composition and fractions of respirable and nonrespirable material were obtained by the elicitation process. 


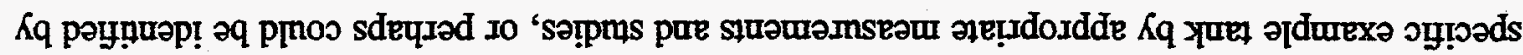

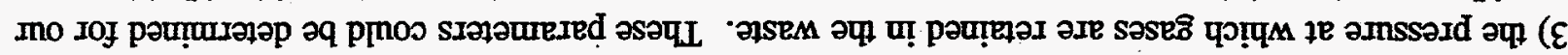

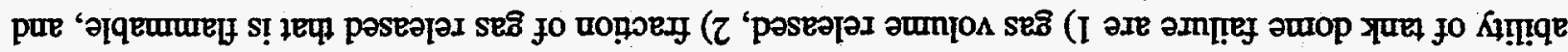

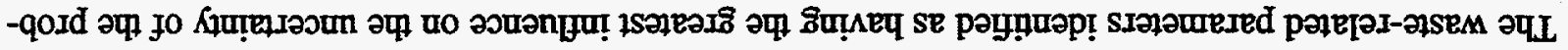

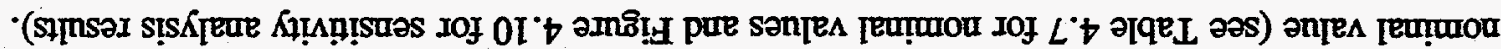

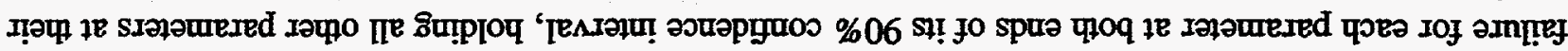

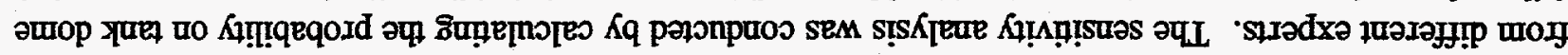

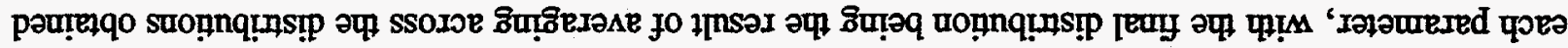
of

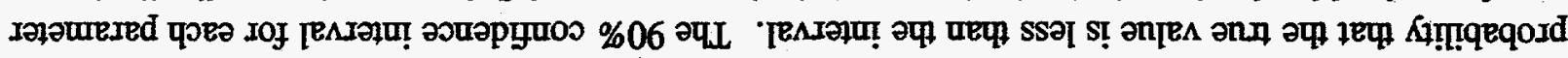

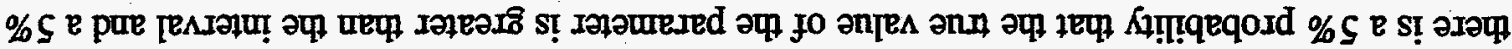

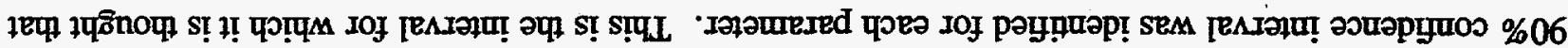

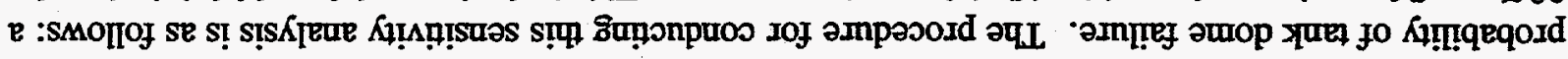

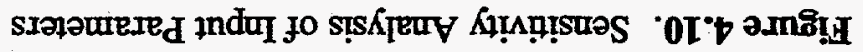

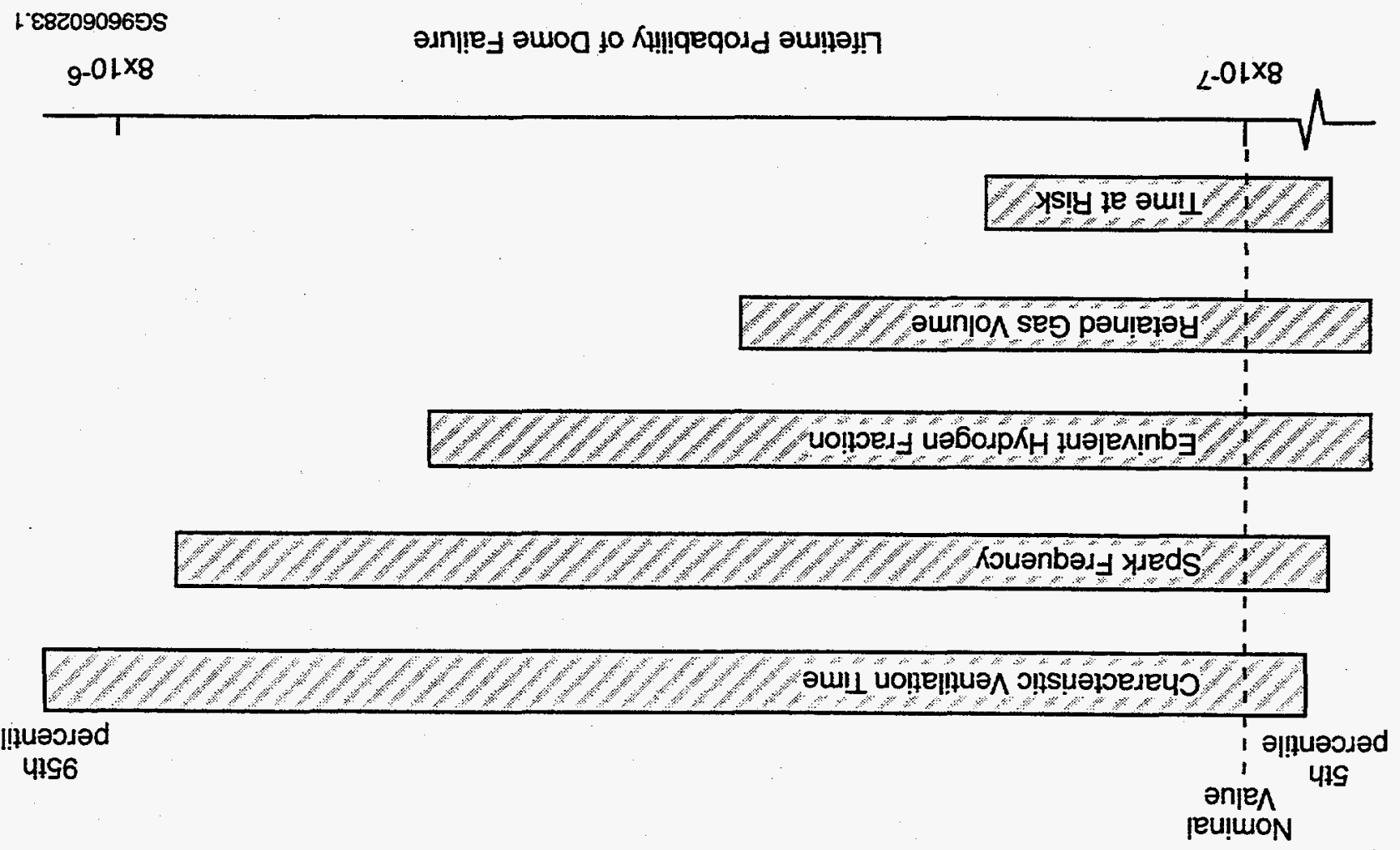


correlation with other tanks with similar wastes. The characteristic ventilation time, a tank system-related parameter, also strongly influences the uncertainty. However, to embark on obtaining tank-specific information for these parameters requires a justified need for the information.

The input parameters obtained by the elicitation process were used to calculate the probability of tank dome failure for this specific tank in its present state. The result is a calculated expected value of

$$
p_{4}=3 \times 10^{-6}
$$

That is, the odds of a dome failure occurring in the lifetime of Tank 241-S-106 are three in one million.

The corresponding expected value for tank dome failure if the mitigation action of installing a permanent active ventilation system is completed is

$$
\mathrm{p}_{4}=1 \times 10^{-8}
$$

which corresponds to odds of one in one hundred million. Both of these values are expected values (i.e., the weighted average of all possible values).

The cumulative distribution for probability of dome failure, based on the uncertainties of the input parameters for the calculation are given in Figure 4.7.

The cumulative distribution of lifetime dome failure probability covers four orders of magnitude for the case of not mitigating. Mitigation by permanent active ventilation reduces the expected value by two orders of magnitude and also reduces the range covered by the cumulative distribution of lifetime dome failure probability. This range in the cumulative distribution of lifetime dome failure probability results from the uncertainties in the input parameters. However, decisions will be made on the basis of risk in comparison to acceptability criteria, not on the basis of probability of occurrence alone.

\subsubsection{The Mitigation Option}

The mitigation option chosen for this example is installing and using a permanent active ventilation system. This option changes the characteristic ventilation rate of the tank. An analysis for effectiveness of the mitigation option shows that the risk is roughly proportional to the characteristic ventilation time, and that permanent ventilation would reduce the characteristic ventilation time by roughly two orders of magnitude; hence, the expected risk could be reduced by two orders of magnitude by the mitigation strategy. The mitigation option considered does not affect the composition of the waste, the composition of the gases produced, or the characteristics of the waste related to the volume of a sudden gas release. Implementation of this mitigation option does not preclude a flammable gas burn; it only impacts the probability of a burn. 
Engineering and cost information for installing an active ventilation system on a single tank existed and was used without change: $\$ 700 \mathrm{~K}{ }^{(a)}$ This cost includes operational and future maintenance costs.

\subsubsection{Justified Information Gathering Option}

Information gathering includes model development and analysis, sampling and analysis of tank contents, and investigation of programmatic decisions and priorities that affect the flammable gas burn probability or consequence in Tank 241-S-106. Figure 4.10 shows that changes in the characteristic ventilation time has the most impact on the probability of dome failure from a flammable gas burn in 241-S-106; thus investigations on this parameter will aid in the decision process if risk acceptance criterion III.b is imposed. Likewise, change in spark frequency has the second most significant impact on the probability of dome failure from a flammable gas burn. Investigations on this parameter will aid in the decision process if risk acceptance criterion II or III.b are imposed.

Changes in equivalent hydrogen fraction and retained gas volume have less impact on the probability of dome failure in 241-S-106. However, information about both parameters relate to the nature of the waste in the tank and would provide insight into the flammable gas burn issue for all of the criteria that may be applied (criteria I, II, and III.b). The remaining parameter, time at risk, is set programmatically. If criterion $I$ is imposed, and information gathered and analyzed about the equivalent hydrogen fraction and/or retained gas volume shows that the gas produced by the waste is flammable, then programmatic issues must be addressed that prioritize the schedule for removal of tank contents within the context of the total risk at the site.

It should be noted that within the scope of performing the analysis on a single tank, information gathering may appear justified. Economies of scale cannot be analyzed on an example of a single tank for a single issue. It is anticipated that over many tanks for a single issue, and over many issues, there will be a cost benefit realized from synergistic learning. The focused learning process that is provided by this strategy is of overall benefit to site issues and corresponding actions.

\subsection{VOI Analysis for Disposal Decisions}

Section 4.2.1 describes the results of a qualitative VOI analysis for disposal decisions. It then outlines a procedure for assessing prior probability distributions over selected tank characteristics. Section 4.2.2 presents a VOI model for TRU segregation and describes the decision analysis for a single tank, presents the VOI model results, and generalizes the application to all TRU tanks. Section 4.2.3 presents a VOI model for the technetium feed specification decision for low-level waste (LLW) feed for Phase I Privatization. This example is developed in terms of a loss function that captures the life-cycle costs and waste volume consequences of the alternatives.

(a) W. Meader, WHC, personal communication. 


\subsubsection{Qualitative Analysis}

A major strength of the VOI approach is the explicit linkage of information to decision making. To establish this link, it is useful to begin the VOI analysis with a survey of the decisions that can be made and a qualitative assessment of the value of different types of tank characterization information to support these decisions. This qualitative analysis points to those decisions which will benefit most from additional tank information and for which a quantitative VOI analysis would be worthwhile.

The qualitative analysis will also identify a limited set of tank waste characteristics that influence disposal decisions in an important way. For example, decisions related to managing the tanks containing TRU wastes are influenced by the knowledge of possible past HLW contamination, the activity level of the TRU waste, and the amount of zirconium in the tank. Looking across disposal decisions in this way will generate a list of tank waste characteristics that have significant decision relevance and those that have little or no decision relevance. A formal assessment of prior probability distributions over these characteristics is the next logical step in a VOI analysis.

This section describes preliminary results of a qualitative VOI analysis for disposal decisions. It also outlines a procedure for assessing prior probability distributions over selected tank characteristics identified as important decision drivers.

To conduct the qualitative VOI analysis, published documents (Johnson 1994, 1996; McConville et al. 1995) and preliminary unpublished lists of decisions were reviewed. These decisions were classified into five categories:

1. Waste retrieval and processing

2. Specifications for privatization

3. Pretreatment and waste form

4. Interim storage and transportation

5. Tank closure and final disposal.

For each of these categories, a preliminary list of decisions was created using available documents. At this stage of the analysis, the list only provides examples of the most important decisions that have to be made in the disposal area. The ultimate goal is to complete lists of decisions for each of the categories.

Having created a list of decisions, the next step of the qualitative VOI analysis is to identify the major issues in each decision problem. These are the uncertainties and tradeoffs that make the decision problem complex. For example, in the problem of whether to keep TRU waste segregated from HLW, the issues are the uncertainties about the volumes and disposal costs of HLW and.TRU wastes and the tradeoff between the waste management complications introduced by segregation versus the possible cost and time savings. 
Next, the characterization information that may be useful for resolving these issues is identified. For example, to resolve the issues related to the segregation of TRU waste from HLW, one would need to know whether the TRU waste may have been contaminated with HLW in the past (in which case it would not make sense to keep it separate), the activity level of the TRU constituents (must be above $100 \mathrm{nCi} / \mathrm{g}$ to be classified as TRU), and the amount of the constituents that determine the glass waste form loading factor, in particular, the amount of zirconium. Additional information not related to the tank contents includes the costs of processing and disposing wastes as TRU rather than HLW.

Finally, a qualitative assessment is made on whether collecting this information would improve the decisions. Three questions are raised in this qualitative assessment:

1. How strongly does the decision depend on the information?

2. If the decision depends on the information, how much can be gained by knowing the information?

3. Does collecting this information require a special effort, or will this information be available with little or no effort prior to making the decision?

To answer the first question in qualitative terms, one classifies the dependency of the decision on the information as none, weak, or strong. For example, the choice of alternative routes to transport HLW to the Yucca Mountain, Nevada, repository does not depend on tank waste information at all. There is only a weak dependency of the choice of retrieval technologies on tank waste information, since retrieval decisions are primarily driven by the retrieval goal rather than by the characteristics and amounts of the sludge. There is a strong dependency of the feed and product specifications for privatization on information about the constituents in the tanks because, with more precise information about the amounts and activity levels of the constituents, feed specifications can be narrowed down (thereby reducing vendors' bids) and product specifications defined more accurately (increasing the likelihood of meeting performance standards).

To answer the second question, one must assess, in approximate terms, what the costs and benefits of each decision are given different states of the information and different prior probabilities. For example, if the cost of segregating TRU waste from HLW is small, and the cost of processing and disposing wastes are significantly lower for TRU wastes than for HLW, it can be worthwhile to determine whether a tank contains TRU waste or HLW. Another consideration is the prior probability of tank waste information. For example, if the prior probability that a tank contains TRU waste is close to 0.50 , it will be worth more to collect the information on whether the waste is TRU than if the prior probability is 0.90 .

The third question addresses the timing and cost of collecting the information for decision making. If the information will be available at no or little cost prior to decision making, it is not worth spending additional effort or expense now to collect it. For example, the final choice of an interim storage facility for HLW can be made after information on waste form performance becomes available. This information is essentially free, and it would not be worth a special effort to collect information for this decision now. 
Tables 4.9 to 4.13 show the preliminary results of this qualitative VOI analysis. For waste retrieval and processing decisions, the value of tank characterization information is assessed to be low to medium. For decisions on privatization specification and for the vendors' decisions about pretreatment and waste form, the VOI is assessed to be high. For interim storage and transportation and for tank closure and final disposal decisions, the VOI is assessed to be low.

Looking across these decisions, several tank waste characteristics appear to have an important impact on disposal decisions:

1. Technicium inventory

2. Chromium, strontium, and cesium inventories

3. TRU inventory

4. Constituents that determine the glass waste form loading factors for HLW, LLW, and TRU wastes.

Table 4.9. Qualitative VOI Analysis for Waste Retrieval and Processing Decisions

\begin{tabular}{|c|c|c|c|}
\hline Decision $^{(a)}$ & Issues & Tank Waste Information & Value of Information \\
\hline $\begin{array}{l}\mathrm{NCAW}^{(\mathbf{a})} \\
\text { consolidation }\end{array}$ & $\begin{array}{l}\text { Create tank space, } \\
\text { safety }\end{array}$ & $\begin{array}{l}\text { Constituents with safety } \\
\text { implications }\end{array}$ & $\begin{array}{l}\text { Low, because there are few associated } \\
\text { safety problems }\end{array}$ \\
\hline $\begin{array}{l}\text { NCRW }^{(b)} \\
\text { consolidation }\end{array}$ & $\begin{array}{l}\text { Create tank space, } \\
\text { avoid contamination } \\
\text { of LLW with TRU }\end{array}$ & $\begin{array}{l}\text { Amount of complexants, } \\
\text { solubility of TRU }\end{array}$ & $\begin{array}{l}\text { Medium, mainly regarding operational } \\
\text { improvements and cost }\end{array}$ \\
\hline $\begin{array}{l}\text { DST retrieval } \\
\text { methods }\end{array}$ & $\begin{array}{l}\text { Efficiency of } \\
\text { retrieval }\end{array}$ & $\begin{array}{l}\text { Amount and characteristics } \\
\text { of sludge }\end{array}$ & $\begin{array}{l}\text { Low, the decisions are driven primar- } \\
\text { ily by the retrieval goal and would not } \\
\text { change with tank information }\end{array}$ \\
\hline $\begin{array}{l}\text { Enhanced } \\
\text { shudge washing }\end{array}$ & $\begin{array}{l}\text { Effects on HLW and } \\
\text { LLW volume, } \\
\text { schedule }\end{array}$ & $\begin{array}{l}\text { Amount and characteristics } \\
\text { of sludge }\end{array}$ & $\begin{array}{l}\text { Low, the decision is less dependent on } \\
\text { tank information than on tests of } \\
\text { enhanced sludge washing }\end{array}$ \\
\hline $\begin{array}{l}\text { Waste retrieval } \\
\text { sequence }\end{array}$ & $\begin{array}{l}\text { Blending, safety, } \\
\text { operational effective- } \\
\text { ness }\end{array}$ & $\begin{array}{l}\text { Amount and activity level of } \\
\text { waste constituents }\end{array}$ & $\begin{array}{l}\text { Medium, value comes largely from } \\
\text { reduced cost and higher effectiveness }\end{array}$ \\
\hline $\begin{array}{l}\text { Segregate TRU } \\
\text { waste and } \\
\text { HLW }\end{array}$ & $\begin{array}{l}\text { Cost, schedule, } \\
\text { effectiveness }\end{array}$ & $\begin{array}{l}\text { HLW contamination of } \\
\text { TRU, activity level of TRU }\end{array}$ & $\begin{array}{l}\text { Medium, value comes from a few } \\
\text { tanks that may have cost savings and } \\
\text { earlier disposal opportunities }\end{array}$ \\
\hline
\end{tabular}


Table 4.10. Qualitative VOI Analysis for Specifications for Privatization

\begin{tabular}{|l|l|l|l||}
\hline \multicolumn{1}{|c|}{ Decision } & \multicolumn{1}{|c|}{ Issues } & \multicolumn{1}{c|}{ Tank Waste Information } & \multicolumn{1}{c|}{ Value of Information } \\
\hline $\begin{array}{l}\text { Feed envelopes } \\
\text { for LLW }\end{array}$ & $\begin{array}{l}\text { Will feed exceed the } \\
\text { envelopes? }\end{array}$ & $\begin{array}{l}\text { Radionuclide inventory by } \\
\text { tank and for all tanks } \\
\text { (especially Tc) }\end{array}$ & $\begin{array}{l}\text { High, drives the vendors' bids and } \\
\text { decisions }\end{array}$ \\
\hline $\begin{array}{l}\text { Product } \\
\text { specifications } \\
\text { for LLW }\end{array}$ & $\begin{array}{l}\text { Can product meet } \\
\text { specifications? }\end{array}$ & $\begin{array}{l}\text { Radionuclide inventory for } \\
\text { all tanks (especially Tc) }\end{array}$ & $\begin{array}{l}\text { High, drives vendors' bids and } \\
\text { ultimate performance characteristics }\end{array}$ \\
\hline $\begin{array}{l}\text { Feed envelopes } \\
\text { for HLW }\end{array}$ & HLW volume? & $\begin{array}{l}\text { Radionuclide inventory by } \\
\text { tank and for all tanks } \\
\text { (especially Cr, Sr, Cs) }\end{array}$ & $\begin{array}{l}\text { High to medium, drives HLW volume } \\
\text { and schedule, may not affect decisions }\end{array}$ \\
\hline $\begin{array}{l}\text { Product } \\
\text { specifications } \\
\text { for HLW }\end{array}$ & $\begin{array}{l}\text { Can product meet } \\
\text { specifications? }\end{array}$ & $\begin{array}{l}\text { Radionuclide inventory for } \\
\text { all tanks (especially Cr, Sr, } \\
\text { Cs) }\end{array}$ & $\begin{array}{l}\text { High, drives vendors' bids and } \\
\text { ultimate performance characteristics }\end{array}$ \\
\hline
\end{tabular}

Table 4.11. Qualitative VOI Analysis for Pretreatment and Waste Form Decisions

\begin{tabular}{||l|l|l|l||}
\hline \multicolumn{1}{|c|}{ Decision } & \multicolumn{1}{|c|}{ Issues } & \multicolumn{1}{c|}{ Tank Waste Information } & \multicolumn{1}{c|}{ Value of Information } \\
\hline $\begin{array}{l}\text { HLW } \\
\text { separation } \\
\text { process }\end{array}$ & $\begin{array}{l}\text { Effectiveness of } \\
\text { separation, cost, } \\
\text { schedule }\end{array}$ & $\begin{array}{l}\text { Total inventories of waste } \\
\text { constituents }\end{array}$ & $\begin{array}{l}\text { High, will influence vendors' } \\
\text { decisions and bids substantially }\end{array}$ \\
\hline $\begin{array}{l}\text { LLW separation } \\
\text { process }\end{array}$ & $\begin{array}{l}\text { Effectiveness of } \\
\text { separation, cost, } \\
\text { schedule }\end{array}$ & $\begin{array}{l}\text { Total inventories of waste } \\
\text { constituents }\end{array}$ & $\begin{array}{l}\text { High, will influence vendors' } \\
\text { decisions and bids substantially }\end{array}$ \\
\hline $\begin{array}{l}\text { Vitrification } \\
\text { concept and } \\
\text { facility }\end{array}$ & $\begin{array}{l}\text { Effectiveness of } \\
\text { meeting product } \\
\text { specs }\end{array}$ & $\begin{array}{l}\text { Total inventories of waste } \\
\text { constituents }\end{array}$ & $\begin{array}{l}\text { High, will influence vendors' } \\
\text { decisions and bids substantially }\end{array}$ \\
\hline Waste form & $\begin{array}{l}\text { Effectiveness of } \\
\text { meeting product } \\
\text { specs }\end{array}$ & $\begin{array}{l}\text { Total inventories of waste } \\
\text { constituents }\end{array}$ & $\begin{array}{l}\text { High, will influence vendors' } \\
\text { decisions and bids substantially }\end{array}$ \\
\hline
\end{tabular}

To pursue a formal VOI analysis, it would be useful to assess prior probability distributions over these variables. Sections 4.2 .2 and 4.2 .3 provide some preliminary assessments of prior probability distributions over TRU activity level in the tanks currently designated as containing TRU and over technicium in the LLW feed. These assessments were made in fairly informal interviews with selected TWRS experts. To create high-quality assessments of these prior probabilities, a more formal elicitation of expert probability judgments would be useful. This type of process has been applied in the nuclear safety and related areas for several years (Merkhofer 1987; Wheeler et al. 1989; Ortiz et al. 1990; Keeney and von Winterfeldt 1991). 
Table 4.12. Qualitative VOI Analysis for Interim Storage and Transportation Decisions

\begin{tabular}{|l|l|l|l||}
\hline \multicolumn{1}{|c|}{ Decision } & \multicolumn{1}{|c|}{ Issues } & \multicolumn{1}{c|}{ Tank Waste Information } & \multicolumn{1}{c|}{ Value of Information } \\
\hline $\begin{array}{l}\text { Choice of } \\
\text { interim storage } \\
\text { facility and site } \\
\text { for LLW }\end{array}$ & $\begin{array}{l}\text { Amount and product } \\
\text { specifications of } \\
\text { LLW, cost, schedule }\end{array}$ & $\begin{array}{l}\text { Tank waste constituents that } \\
\text { determine the amount of } \\
\text { LLW }\end{array}$ & $\begin{array}{l}\text { Low, decisions can be made after } \\
\text { obtaining LLW product }\end{array}$ \\
\hline $\begin{array}{l}\text { Choice of } \\
\text { interim storage } \\
\text { facility and site } \\
\text { for HLW }\end{array}$ & $\begin{array}{l}\text { Amount and product } \\
\text { specifications of } \\
\text { HLW, cost, } \\
\text { schedule }\end{array}$ & $\begin{array}{l}\text { Tank waste constituents that } \\
\text { determine the amount of } \\
\text { HLW }\end{array}$ & $\begin{array}{l}\text { Low, decisions can be made after } \\
\text { obtaining HLW product }\end{array}$ \\
\hline $\begin{array}{l}\text { Choice of } \\
\text { interim storage } \\
\text { facility and site } \\
\text { for TRU waste }\end{array}$ & $\begin{array}{l}\text { Amount and product } \\
\text { specifications of } \\
\text { TRU, cost, schedule }\end{array}$ & $\begin{array}{l}\text { Tank waste constituents that } \\
\text { determine the amount of } \\
\text { TRU }\end{array}$ & $\begin{array}{l}\text { Low, decisions can be made after } \\
\text { obtaining TRU product }\end{array}$ \\
\hline $\begin{array}{l}\text { Disposition and } \\
\text { storage of } \\
\text { separated Cs, } \\
\text { Tc, Sr, TRU }\end{array}$ & $\begin{array}{l}\text { Volume and activity } \\
\text { level of separated } \\
\text { Tc, Cs, Sr, TRU }\end{array}$ & $\begin{array}{l}\text { Inventory of Tc, Sr, Cs, } \\
\text { TRU in tanks }\end{array}$ & $\begin{array}{l}\text { Low, decisions can be made after } \\
\text { determining amounts }\end{array}$ \\
\hline $\begin{array}{l}\text { Mode and route } \\
\text { of shipping } \\
\text { HLW and TRU } \\
\text { canisters }\end{array}$ & $\begin{array}{l}\text { Number and types of } \\
\text { HLW \& TRU } \\
\text { canisters }\end{array}$ & $\begin{array}{l}\text { Tank waste constituents that } \\
\text { determine the amount of } \\
\text { HLW and TRU wastes }\end{array}$ & $\begin{array}{l}\text { Low, decisions can be made after } \\
\text { determining amounts of HLW and } \\
\text { TRU wastes }\end{array}$ \\
\hline
\end{tabular}

Table 4.13. Qualitative VOI Analysis for Tank Closure and Final Disposal Decisions

\begin{tabular}{||l|l|l|l||}
\hline \multicolumn{1}{|c|}{ Decision } & \multicolumn{1}{|c|}{ Issues } & \multicolumn{1}{c|}{ Tank Waste Information } & \multicolumn{1}{c|}{ Vahe of Information } \\
\hline $\begin{array}{l}\text { Strategy for tank } \\
\text { closure }\end{array}$ & $\begin{array}{l}\text { Amount and activity } \\
\text { levels of residuals }\end{array}$ & $\begin{array}{l}\text { Amounts and activity levels } \\
\text { of major radionuclides in } \\
\text { sludge }\end{array}$ & $\begin{array}{l}\text { Low, decisions can be made after } \\
\text { determining amounts }\end{array}$ \\
\hline $\begin{array}{l}\text { Final disposal } \\
\text { site for HLW }\end{array}$ & $\begin{array}{l}\text { Is Yucca Mountain } \\
\text { available? }\end{array}$ & NA & $\begin{array}{l}\text { None, decision driven by external } \\
\text { factors }\end{array}$ \\
\hline $\begin{array}{l}\text { Final disposal } \\
\text { site for TRU }\end{array}$ & $\begin{array}{l}\text { Will TRU be WIPP } \\
\text { certified? }\end{array}$ & $\begin{array}{l}\text { Constituents that determine } \\
\text { the amount and activity } \\
\text { levels of vitrified TRU }\end{array}$ & $\begin{array}{l}\text { Low, decision driven by external } \\
\text { factors }\end{array}$ \\
\hline
\end{tabular}


As mentioned earlier, eliciting prior probability distributions can build on existing work. For example, McConville et al. (1994) provides first cut prior probability distributions in the form of three point approximations. In addition, substantial work exists on historical records, simulations, and actual samples.

\subsubsection{VOI Model for TRU Segregation Decision}

DOE Order 5820.2A defines HLW as highly radioactive material that results from the reprocessing of spent nuclear fuel, including liquid waste produced directly in reprocessing and any solid waste derived from the liquid, that contains a combination of TRU and fission products in concentrations requiring permanent isolation. TRU is defined as waste that is contaminated with alpha-emitting transuranic radionuclides with half-lives greater than 20 years and concentrations greater than $100 \mathrm{nCi} / \mathrm{g}$. Low-level waste (LLW) is defined as waste that contains radioactivity and is not classified as HLW, transuranic waste, spent nuclear fuel, or byproduct material.

Most of the 177 tanks at the Hanford Site contain HLW. There are 10 tanks that are likely to contain TRU wastes:

$\begin{array}{lll}241-B-201 & 241-T-201 & 241-A W-103 \\ 241-B-202 & 241-T-202 & 241-A W-105 \\ 241-B-203 & 241-T-203 & \\ 241-B-204 & 241-T-204 & \end{array}$

Two additional tanks, 241-T-110 and 241-T-111, are likely to contain either TRU wastes or LLW rather than HLW.

Three tanks may contain TRU wastes, but they are probably contaminated with HLW; they are 241-B-110, 241-B-111, and 241-B-112.

The decision problem is whether to keep the wastes from these 15 tanks segregated from the HLW stream or to blend them with the HLW. Arguments for segregation are the lower cost of processing and disposing TRU wastes (about $\$ 60,000 / \mathrm{MT}$ ) and the fact that a TRU waste disposal site (WIPP) exists and is likely to be licensed in the near future. In contrast, HLW processing and disposal costs are higher (about $\$ 160,000 / \mathrm{MT}$ ) and there is considerable uncertainty about whether the nation's HLW repository at Yucca Mountain will ever be licensed. Arguments for blending are the increased operational and dollar costs of segregation, the lower loading factors of TRU wastes (which increase its volume relative to HLW), and questions about the availability of space at the WIPP site.

For the two tanks that could possibly be classified as containing LLW, the arguments for segregation are somewhat stronger, since the costs of LLW processing and disposal are much lower (about $\$ 400 / \mathrm{MT}$ ) 
and the onsite disposal of LLW has significantly fewer licensing and certification problems than TRU or HLW disposal. However, the likelihood that the wastes in these two tanks will be classified as LLW is quite low.

This VOI analysis begins with a decision analysis for a single tank to determine whether the waste from this tank should be segregated from the HLW stream. The sensitivity of the decision to prior probabilities and other estimates is shown. Next, the results of an analysis of the EVPI and the EVSI are presented. Finally, generalizations of this analysis to other tanks are presented.

\subsubsection{Decision Analysis for a Single Tank}

The decision tree for this problem is shown in Figure 4.11. This analysis is for Tank T-111, with $1850 \mathrm{MT}$ of waste that could either be HLW, TRU, or LLW. There are three initial alternatives for this tank: blend the waste with the HLW stream, segregate the waste as TRU waste, or segregate the waste as LLW. If the waste is blended with the HLW stream, the issue of whether the waste actually could have been classified as TRU or LLW becomes moot. The only uncertainties are the loading factor of the (blended) HLW and the cost of processing and disposing of the waste as HLW (see the upper part of the decision tree). The loading factor for blended $\mathrm{HLW}$ is close to 0.50 (i.e., for each unit of waste, there will be twice as much volume of glass produced). In this analysis, the continuous probability distribution over the loading factor is approximated by a three-point distribution that assigns a probability of 0.185 to the $5^{\text {th }}$ fractile of the continuous distribution, 0.630 to the median, and 0.185 to the $95^{\text {th }}$ fractile (Keefer and Bodily 1983). Similarly, the continuous distribution over the cost of processing and disposing of $\mathrm{HLW}$ is approximated by a three-point distribution.

If the waste is initially segregated and treated as TRU waste, further investigation may determine that the waste was, at some point in time, contaminated by HLW. This information is likely to come from an examination of historical records, since the definition of HLW contamination is related to the source of the waste, not the amounts and activity levels of the radionuclides. If the waste turns out to be contaminated with HLW, it would, of course, need to be included in the HLW stream. In that case, the remaining uncertainties are the loading factor of HLW and the cost of HLW processing and disposal. If the waste turns out to be TRU, it would be processed separately and treated and disposed of as TRU waste. The remaining uncertainties are the loading factor of TRU and the cost of processing the waste as TRU. As with the HLW case, the continuous probability distributions about the loading factor and cost are approximated by three-point distributions.

For Tank 241-T-111, there is an additional opportunity to treat the waste initially as LLW. Further investigation will reveal whether the waste was contaminated by HLW. If it was contaminated it would be included in the HLW stream, and the subsequent uncertainties are the loading factor and cost of HLW processing and disposal. If the waste was not contaminated, there is a possibility that it is TRU ( $>100 \mathrm{nCi} / \mathrm{g})$ or $L L W(<100 \mathrm{nCi} / \mathrm{g})$. The resolution of this uncertainty would require sampling of the tank waste. If the 


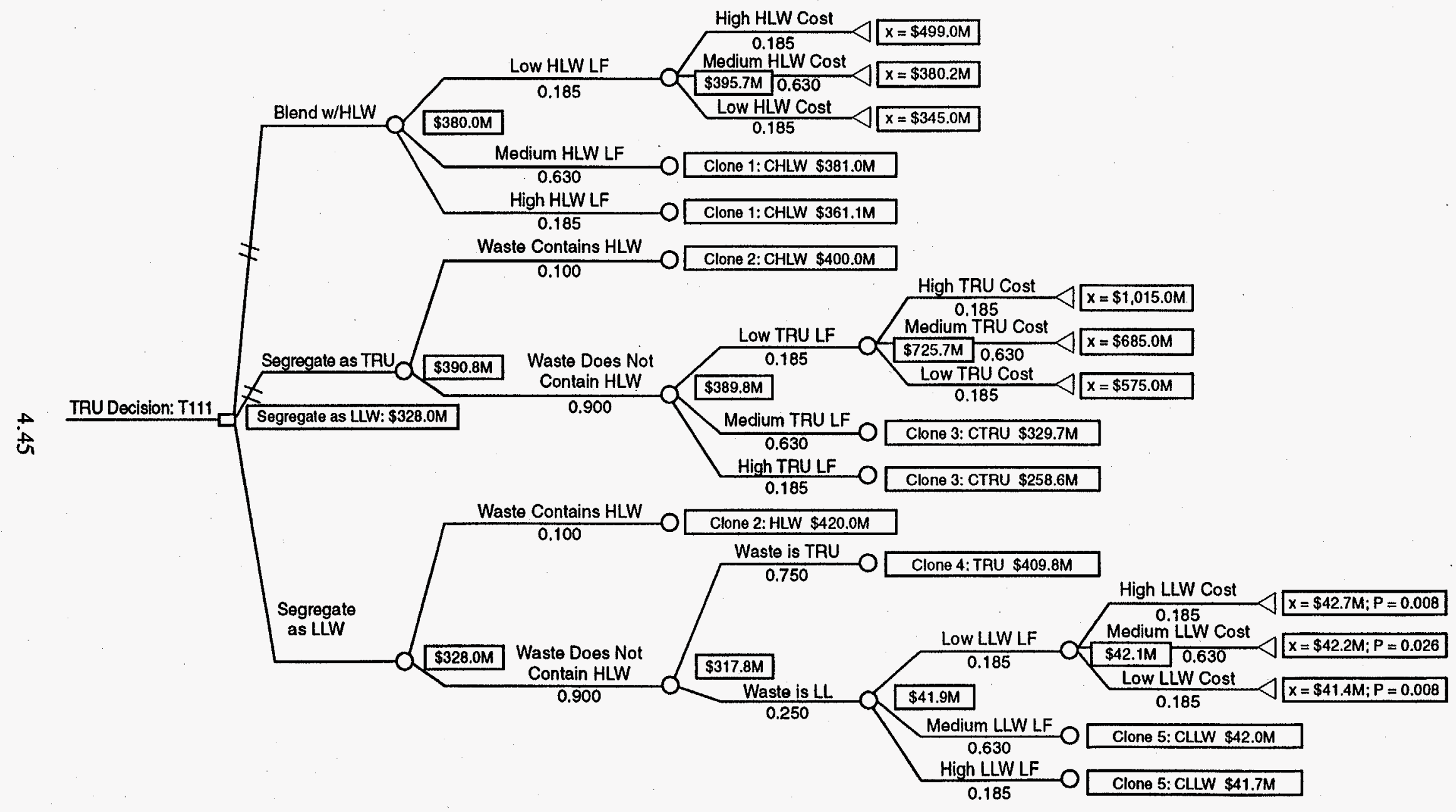

Figure 4.11. Decision Tree for TRU Segregation Problem 
waste turns out to be TRU it would enter the TRU waste stream, and the subsequent uncertainties concern the TRU loading factor and processing and disposal costs. If the waste turns out to be LLW it would enter the LLW stream, and the remaining uncertainties are about the loading factor and costs of LLW processing and disposal. As with the HLW and TRU cases, the continuous distributions about the loading factor and the cost are approximated by three-point distributions.

At the end of each path through the decision tree there will be consequences, which are evaluated in terms of three objectives:

1. Minimize waste processing and disposal costs

2. Minimize costs and hassles of segregating wastes

3. Minimize time to final disposal:

These objectives are measured by the following attributes:

1. Total life-cycle cost for processing and disposal for one MT of waste.

2. Fixed cost for segregating TRU from HLW and for segregating LLW from TRU/HLW. This fixed cost is parameterized in the model.

3. For each year after the year 2000 , a fixed cost penalty is applied for late disposal. This penalty is parameterized in the model.

For Tank 241-T-111, in this example, the following probabilities were assessed in discussions with experts at Westinghouse Hanford Company:

Probability of no contamination with $\mathrm{HLW}=\mathrm{p}=\mathbf{0 . 9 0}$

Probability of contamination with HLW $=1-p=0.10$

Probability of TRU, given no contamination with $\mathrm{HLW}=\mathrm{q}=0.75$

Probability of $L L W$, given no contamination with $H L W=1-q=0.25$.

The probability distributions over loading factors and costs were assessed through interviews with experts at WHC and the Pacific Northwest National Laboratory. The preliminary assessments for the glass loading factors used in this model are shown in Table 4.14.

At the end of each path through the decision tree, consequences must be estimated in terms of three attributes: 1) the cost of processing and disposal, 2) the cost of segregation, and 3) the cost of delaying final disposal. The cost of processing and disposal for HLW is calculated as

$$
C D=(T W / H L W L F) * C H L W
$$


Table 4.14. Probability Distributions (fractiles) for Glass Loading Factors

\begin{tabular}{||l|l|c|}
\hline \multicolumn{1}{|c|}{ Name } & \multicolumn{1}{|c|}{ Description } & Base Value \\
\hline HLWLF05 & Low HLW loading factor & 0.50 \\
\hline HLWLF50 & Medium HLW loading factor & 0.52 \\
\hline HLWLF95 & High HLW loading factor & 0.55 \\
\hline TRULF05 & Low TRU loading factor & 0.10 \\
\hline TRULF50 & Medium TRU loading factor & 0.23 \\
\hline TRULF95 & High TRU loading factor & 0.30 \\
\hline LLWLF05 & Low LLW loading factor & 0.20 \\
\hline LLWLF50 & Medium LLW loading factor & 0.22 \\
\hline LLWLF95 & High LLW loading factor & 0.25 \\
\hline
\end{tabular}

where TW is the mass of the tank waste to be vitrified (in MT), HLWLF is the loading factor, and CHLW is the cost per MT of processing and disposing of HLW. Similar equations apply to TRU and LLW. To obtain the MT of waste requiring vitrification for each tank, the tank waste volume was transformed into metric tons, and a reduction factor was applied to reflect the effectiveness of sludge washing and leaching. Estimates of this mass reduction factor vary from $10 \%$ to $90 \%$, depending on the constituents of the waste in each tank and the assumptions made about the method and effectiveness of pretreatment. For this preliminary analysis, a moderate mass reduction of $40 \%$ was assumed. Table 4.15 shows the distribution of costs per MT for HLW, TRU, and LLW.

The cost of segregation is parameterized, since it is very difficult to provide firm estimates. The assumption is made that the cost of segregation is a fixed penalty (possibly depending on the tank volume) which relates to the additional management and operations difficulties of keeping the wastes in this tank separate from other tanks. Furthermore, the assumption is made that the cost of segregation of TRU waste from HLW is less (base case: $\$ 10$ million for small tanks with less than $100 \mathrm{kgal}$ volume, $\$ 20$ million for large tanks with more than $100 \mathrm{kgal}$ volume) than the cost of segregating a potentially LLW tank from TRU and HLW (base case: $\$ 20$ million for small tanks, $\$ 40$ million for large tanks). Since Tank $241-T-111$ is considered a large tank, penalties of $\$ 20$ million and $\$ 40$ million were assigned to its segregation as a TRU and LLW tank, respectively.

The cost of delayed final disposal was also parameterized. The earliest disposal could occur with LLW in about 2005 . With TRU waste, the disposal could occur early as well. However, to indicate the additional complexities with obtaining licenses and certifications of TRU waste for disposal at WIPP, the base case estimate for TRU waste was set to be 2010 (i.e., a five-year delay relative to LLW disposal). 
Table 4.15. Probability Distributions (fractiles) for Cost (per MT)

\begin{tabular}{|l|l|c||}
\hline \multicolumn{1}{|c|}{ Name } & \multicolumn{1}{c|}{ Description } & Base Value \\
\hline CHLW05 & Low HLW disposal cost & $\$ 150,000$ \\
\hline CHLW50 & Medium HLW disposal cost & $\$ 166,000$ \\
\hline CHLW95 & High HLW disposal cost & $\$ 220,000$ \\
\hline CTRU05 & Low TRU disposal cost & $\$ 50,000$ \\
\hline CTRU50 & Medium TRU disposal cost & $\$ 60,000$ \\
\hline CTRU95 & Hligh TRU disposal cost & $\$ 90,000$ \\
\hline CLLW05 & Low LLW disposal cost & $\$ 250$ \\
\hline CLLW50 & Medium LLW disposal cost & $\$ 400$ \\
\hline CLLW95 & High LLW disposal cost & $\$ 500$ \\
\hline
\end{tabular}

There currently is no HLW repository, and even the Secretary of Energy has stated that the chance that the Yucca Mountain will eventually be licensed as the nation's first HLW repository is only about 50 percent. To indicate the difficulty and delay in the final disposal of HLW, the base case assumes a final disposal date of 2020. Each year of delay after 2005 is penalized with a cost of $\$ 1,000,000$.

Value function. To aggregate processing and disposal costs, the cost of segregation, and years of delay in final disposal, a simple additive value function was used:

$$
\mathrm{X}=\mathrm{CD}+\mathrm{CSEG}+(\mathrm{YEAR}-2000) * \mathrm{CPY}
$$

where $\mathrm{X}$ is the total cost, CD is the cost of processing and disposal, CSEG is the cost of segregating the wastes, YEAR is the year of final disposal, and CPY is the penalty for each year of delay in disposing of the waste.

Base Case and Sensitivity Analysis. Figure 4.11 shows the "rolled back" decision tree with the expected equivalent costs calculated at each node. It shows that for this tank, the best decision is to keep it segregated as a potential LLW tank, with an expected equivalent cost of $\$ 328.0$ million. The alternative to blend the tank waste with the HLW stream has an expected equivalent cost of segregating the tank as TRU has the highest expected equivalent cost of $\$ 390.8$ million.

Table 4.16 shows the equivalent costs broken down into the three attributes of this analysis: direct cost of processing and disposal, cost of segregation, and cost of delay of final disposal. The main benefit from 
Table 4.16. Expected Equivalent Cost (in \$ Millions) for TRU Segregation Alternatives

\begin{tabular}{||l|c|c|c||}
\hline \multirow{2}{*}{\multicolumn{1}{|c|}{ Attribute }} & \multicolumn{3}{c|}{ Alternatives } \\
\cline { 2 - 4 } & Blend with HLW & Segregate as TRU & Segregate as LLW \\
\hline \hline Processing and disposal cost & 365.0 & 364.8 & 283.2 \\
\hline Segregation cost & 0.0 & 20.0 & 40.0 \\
\hline Cost of delay of final disposal & 15.0 & 6.0 & 4.8 \\
\hline Total cost & 380.0 & 390.8 & 328.0 \\
\hline
\end{tabular}

treating the tank waste as LLW is due to the chance ( $\mathrm{pxq}=0.225$ ) that the waste will eventually be classified as LLW. The direct expected costs of processing the waste as HLW versus TRU waste are very similar. Yet the decision to blend the waste with HLW is preferred because the cost of segregation is large compared with penalties for delays.

To further analyze how the differences in total cost depend on the cost of segregation and the penalties for later disposal, a sensitivity analysis on these two variables was performed (see Figure 4.12). It shows that only for extremely high costs of segregation ( $\$ 80$ million and above) and low costs of delays, the best decision is to blend this tank's waste with the HLW stream. Otherwise, the waste should be segregated as LLW.

Figure 4.13 shows how the equivalent expected costs of the three alternatives vary as a function of $p$, the probability that the waste was not contaminated with HLW. For relatively low probabilities of less than 0.4 (indicating a good chance of contamination), the best decision is to blend the tank waste with HLW. For probabilities above 0.4 , the best decision is to treat the tank as LLW.

Figure 4.14 shows a two-way sensitivity analysis of $p$ (probability that the tank waste is not contaminated with $\mathrm{HLW}$ ) and $\mathrm{q}$ (probability that the tank waste is TRU, given that it is not contaminated with $H L W$ ). It shows that for most combinations of $p$ and $q$, the best decision is to segregate the tank as LLW. If $p$ is low and $q$ is high (which is likely to be the case for most tanks except 241-T-110 and 241-T-111), the best decision is to blend the tank waste with HLW.

\subsubsection{TRU Segregation Model Results}

Table 4.17 shows the results of various EVPI analyses. The value of knowing whether the waste has been contaminated with HLW is worth $\$ 4.0$ million, and the value of knowing whether the waste is TRU or low level is $\$ 23.0$ million. The combined VOI of knowing both probabilities is $\$ 24.0$ million. The VOI about the loading factors is also very high ( $\$ 32.3$ million) because there is substantial uncertainty about the 


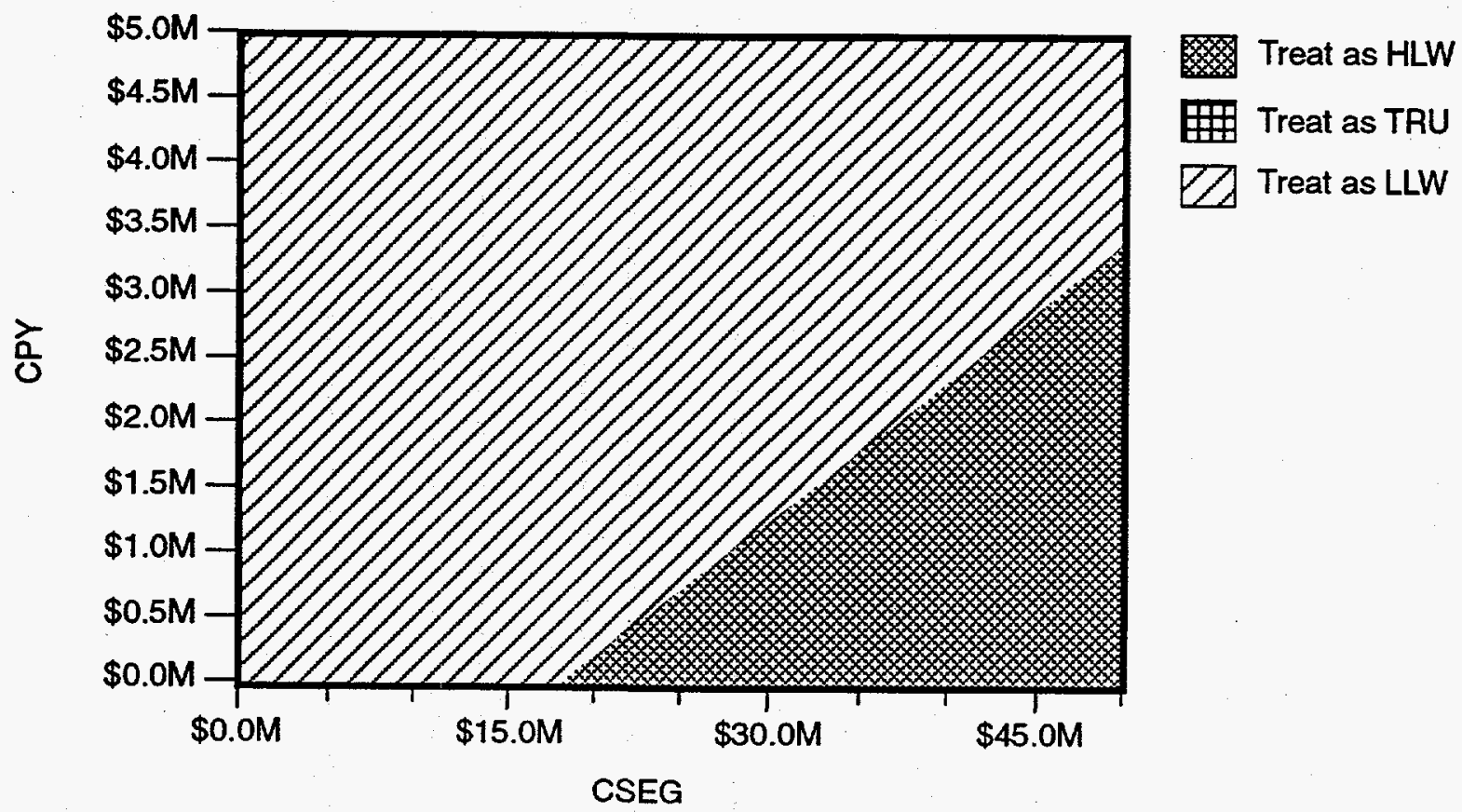

SG96100412.4

Figure 4.12. Sensitivity Analysis on Cost of Segregation (CSEG) and Penalty for Late Disposal (CPY)

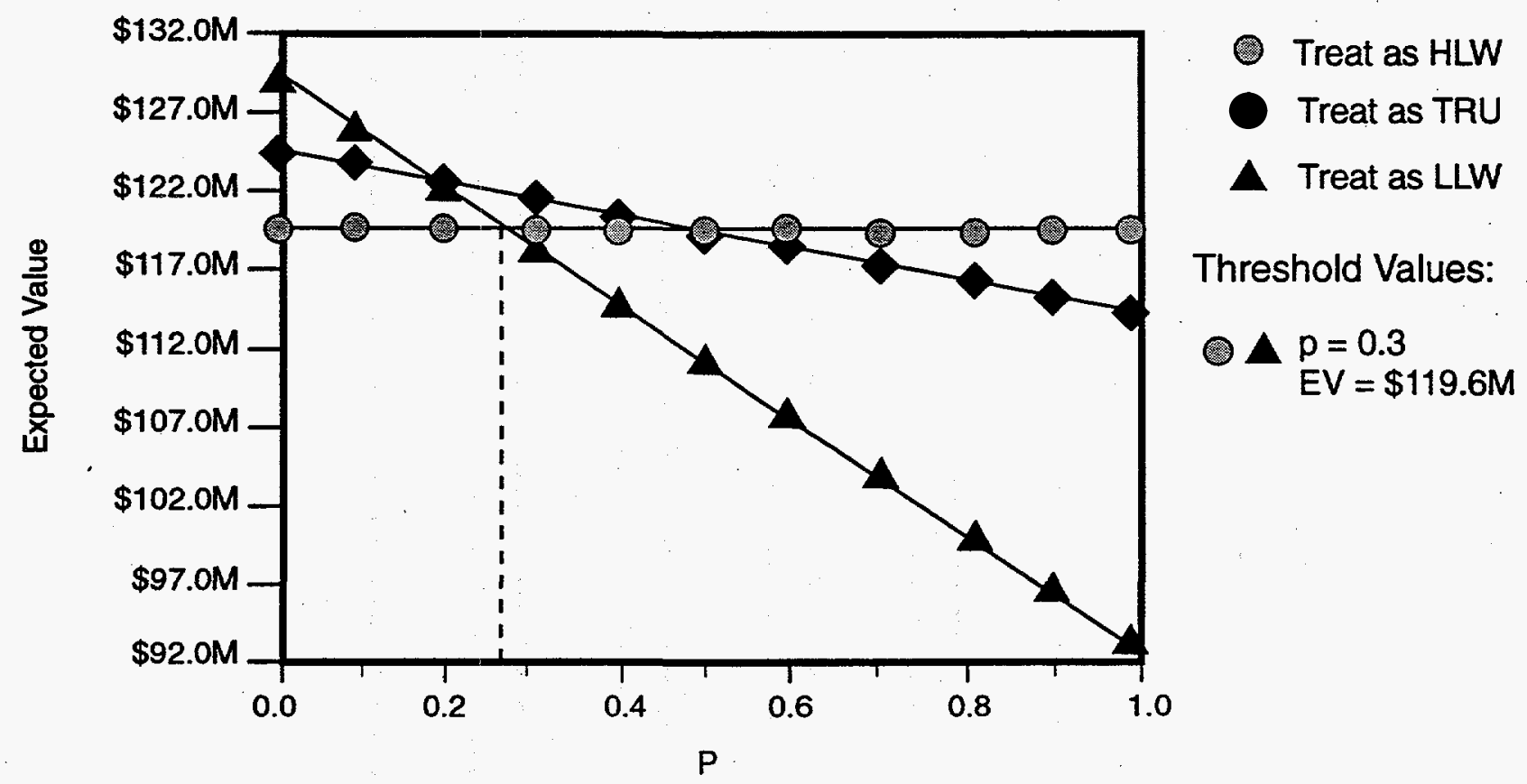

SG96100412.2

Figure 4.13. Sensitivity Analysis on the Probability That Tank 241-T-111 Is Not Contaminated with HLW 


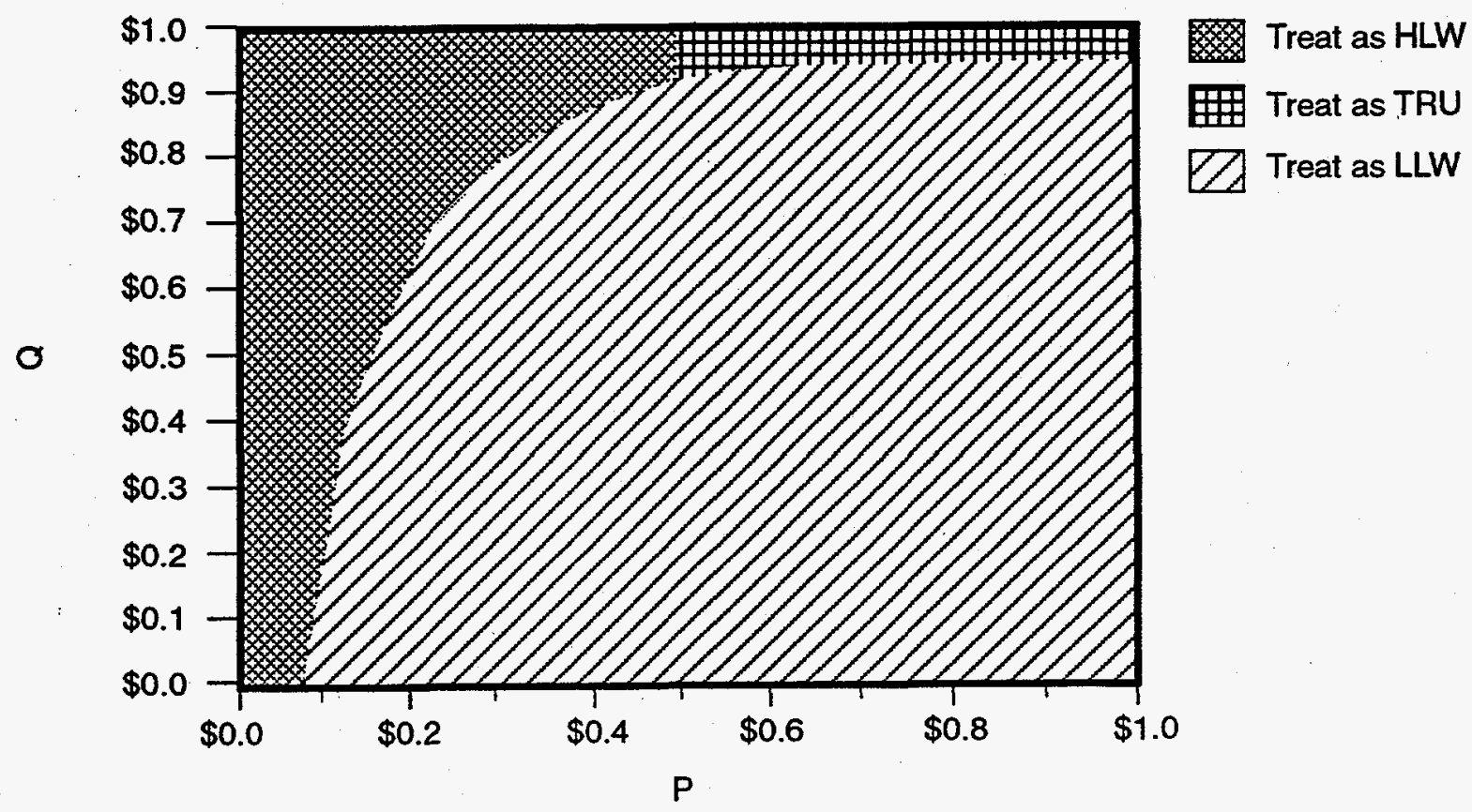

SG96100412.3

Figure 4.14. Two-Way Sensitivity Analysis on $p$ and $q$

loading factor of TRU, which creates a substantial uncertainty about the cost of TRU processing and disposal. On the other hand, the value of knowing the unit cost of processing and disposal is lower ( $\$ 10.5$ million), because the range of costs provided by the experts was fairly narrow.

Given the fairly high values of perfect information for all variables, it is worth investigating selected characterization plans for Tank $\mathrm{T}-111$. There are three sources of information that can be obtained to resolve the uncertainties:

1. Study of historical records to determine past HLW contamination - resolves uncertainty about p.

2. Taking one or more core samples to determine the exact activity level of the TRU waste - resolves uncertainty about $\mathrm{q}$.

3. Taking one or more core samples to determine the amount of zirconium in the tank - resolves uncertainty about the loading factors for TRU.

Table 4.18 illustrates the assumed diagnosticity of collecting historical information. The table shows the conditional probabilities of results of the evidence thus collected, given the true state about HLW contamination. The assumption is made that if there was no HLW contamination, the evidence will rarely suggest such contamination by mistake. However, if there was some HLW contamination, the evidence 
Table 4.17. Expected Value of Perfect Information Analyses (Summary)

\begin{tabular}{||l|c|c|}
\hline Perfect Information About & EV (preferred alternative) in millions & EVPI (in millions) \\
\hline $\mathrm{p}$ and $\mathrm{q}$ & $\$ 304.0$ & $\$ 24.0$ \\
\hline $\mathrm{P}$ & $\$ 324.0$ & $\$ 4.0$ \\
\hline $\mathrm{q}$ & $\$ 305.0$ & $\$ 23.0$ \\
\hline Loading Factors & $\$ 295.7$ & $\$ 32.3$ \\
\hline Cost & $\$ 317.5$ & $\$ 10.5$ \\
\hline
\end{tabular}

Table 4.18. Diagnosticities for Imperfect Information

Diagnosticities for Historical Information about "Contains $H L W$ "versus "Does Not Contain $H L W$ "

\begin{tabular}{|l|c|c|}
\hline \multirow{2}{*}{} & \multicolumn{2}{|c|}{ True State } \\
\cline { 2 - 3 } & Contains HLW & Does Not Contain HLW \\
\hline \hline \multirow{2}{*}{ Evidence suggests HLW } & 0.60 & 0.90 \\
\hline Evidence does not suggest HLW & 0.40 & 0.10 \\
\hline
\end{tabular}

Diagnosticities for Historical Information about "Waste is TRU"versus "Waste is $L L W "$

\begin{tabular}{|l|c|c|}
\hline & Waste is TRU & Waste is LLW \\
\hline Sample is TRU & 0.90 & 0.05 \\
\hline Sample is LLW & 0.10 & 0.95 \\
\hline
\end{tabular}

will be somewhat inconclusive. These assumptions reflect the weak nature of historical records to establish HLW contamination. There always will be debate about this issue, no matter how detailed the records are and how much they are studied.

Due to the weak diagnosticity, if there has been HLW contamination, the expected value of imperfect historical information is $\$ 0.0$ million (as opposed to a value of perfect information of $\$ 4.0$ million). Thus, given the questionable accuracy of historical information, it is not worthwhile to conduct a more detailed analysis records for this tank. 
Table 4.18 also shows the assumed diagnosticity of taking one core sample to determine whether the tank waste is TRU ( $>100 \mathrm{nCi} / \mathrm{g}$ ) or LLW $(<100 \mathrm{nCi} / \mathrm{g})$. These diagnosticities were elicited informally with a TWRS expert. If the waste is actually LLW, the sample will be very diagnostic: there is almost no chance of a false classification as TRU. On the other hand, if the waste is TRU, then the probability of classifying it correctly as TRU is only 0.90 versus classifying it incorrectly as LLW $(0.10)$.

Even though the diagnosticity of one core sample is not perfect, the value of sample analysis shows that it is worth spending $\$ 17.0$ million on obtaining this information. This would justify taking several core samples for this tank at a cost of $\$ 1$ million each. These sample should also be analyzed for the amounts and activity levels of zirconium to re-assess the uncertainties about the loading factors for TRU. Since the VOI about zirconium is high, it is likely that taking several core samples may be worthwhile.

\subsubsection{Applications to All TRU Tanks}

Table 4.19 shows current estimates of waste volumes and probabilities for the 15 suspected TRU tanks. The second column lists the volume of solid wastes in these tanks according to Hanlon (1995). Six tanks (241-AW-13, -AW-105, 241-T-110, 241-T-111, 241-B-110, and 241-B-111) contain more than 85\% of the waste volume. The third column of Table 4.19 summarizes preliminary ranges of the probability $\mathrm{p}$ that the tank waste has not been contaminated by HLW. The fourth column shows the preliminary ranges of the probability $\mathrm{q}$ that the waste is TRU, given that it is not contaminated with HLW. These probability estimates were obtained via interviews with tank waste experts at the Hanford Site.

Tanks 241-B-201 to 241-B-204 and 241-T-201 to 241-T-204 have a fairly small volume of waste (21 to $51 \mathrm{kgal}$ each). They have a high probability of not being contaminated with HLW and a very high probability of being TRU, given that they are not contaminated with HLW. Thus, they are most likely classified as TRU tanks. Tanks 241-AW-103 and 241-AW-105 contain large volumes of NRCW wastes, and they are likely to be TRU if they have not been contaminated with HLW. However, there has been considerable debate about the possibility that HLW contamination occurred for these tanks at some point in time. The two tanks 241-T-110 and 241-T-111 are special in that they contain large volumes of wastes that are probably not contaminated by $\mathrm{HLW}$ and have a fairly good chance (about 0.25 ) to be classified as LLW or incidental wastes. Tanks 241-B-110 to 241-B-112 have a good chance of having been contaminated with HLW, but if they have not been contaminated, they are more likely to contain TRU waste than LLW.

As in the example of Tank 241-T-111, the waste volume (in kgal) of each tank was first transformed into a mass (in MT) of in-tank waste. Next, a mass reduction factor was applied to take into account the effectiveness of in-tank washing, leaching, and other methods of pretreatment. Estimates of this mass reduction factor vary from $10 \%$ to $90 \%$, depending on the constituents of the waste in each tank and the assumptions made about the method and effectiveness of pretreatment. For this preliminary analysis, a moderate mass reduction of $40 \%$ was assumed for all tanks. However, we also conducted some sensitivity 
Table 4.19. Application to All TRU Tanks

\begin{tabular}{|c|c|c|c|}
\hline Tank & Volume in kgal & $\begin{array}{l}\text { p: Probability of no } \\
\text { contamination with HLW }\end{array}$ & $\begin{array}{l}\text { q: Probability of TRU given } \\
\text { no contamination with HLW }\end{array}$ \\
\hline 241-B-201 & 29 & $>0.90$ & $>0.95$ \\
\hline 241-B-202 & 27 & $>0.90$ & $>0.95$ \\
\hline 241-B-203 & 51 & $>0.90$ & $>0.95$ \\
\hline 241-B-204 & 50 & $>0.90$ & $>0.95$ \\
\hline $241-\mathrm{T}-201$ & 29 & $>0.90$ & $>0.95$ \\
\hline $241-\mathrm{T}-202$ & 21 & $>0.90$ & $>0.95$ \\
\hline $241-T-203$ & 35 & $>0.90$ & $>0.95$ \\
\hline $241-\mathrm{T}-204$ & 38 & $>0.90$ & $>0.95$ \\
\hline 241-AW-103 & 363 & $\sim 0.50$ & $>0.95$ \\
\hline 241-AW-105 & 297 & $\sim 0.50$ & $>0.95$ \\
\hline 241-T-110 & 376 & $>0.90$ & $\sim 0.75$ \\
\hline 241-T-111 & 297 & $>0.90$ & $\sim 0.75$ \\
\hline 241-B-110 & 245 & $<0.50$ & $>0.95$ \\
\hline 241-B-111 & 237. & $<0.50$ & $>0.95$ \\
\hline 241-B-112 & 33 & $<0.50$ & $>0.95$ \\
\hline
\end{tabular}

analyses to assess the impact of different pretreatment efficiencies for tanks where such information was available. The qualitative recommendations were not altered in a substantial way by using different mass reduction factors.

Next, the loading factors in Table 4.14 were applied for all tanks to determine the mass of vitrified waste product. Applying these factors uniformly for all tanks is reasonable, if the wastes are blended with other wastes. However, if one wants to examine the loading factors for a particular tank without blending its waste with other tanks, these factors will need to be adjusted to reflect the particular limiting constituents of each tank.

The mass of the vitrified product was then multiplied with the costs per metric ton shown in Table 4.15 to determine the ultimate processing and disposal costs for each tank. The segregation costs for the TRU 
were assumed to be $\$ 10$ million for the small-volume tanks ( $>100 \mathrm{kgal}$ ) and $\$ 20$ million for the largevolume tanks ( $>100 \mathrm{kgal}$ ). Similarly, the segregation cost for LLW was assumed to be $\$ 20$ million for small tanks and $\$ 40$ million for large tanks. The years of disposal were the same for all tanks: 2005 for LLW, 2010 for TRU, and 2020 for HLW.

Using this information, the EVPI was calculated for all 15 tanks. Column 2 of Table 4.20 shows the EVPI of $p$, the probability that there has not been any HLW contamination. The EVPI is zero for all but Tanks 241-T-110 and 241-T-111. The EVPI for q, the probability that a tank contains TRU waste (versus LLW), given that it does not contain HLW, is moderate (around \$0.6 to \$5.8 million) for all tanks except for 241-T-110 and 241-T-111, for which the EVPI is high (about $\$ 23$ million). This reflects the fact that these two tanks have a potential for containing LLW, which would significantly reduce the cost of processing and disposal.

Table 4.20. Preliminary VOI Results and Recommendations (\$M)

\begin{tabular}{||c|c|c|c|c|c||}
\hline \multirow{2}{*}{ Tank } & \multicolumn{2}{|c|}{$\begin{array}{c}\text { Expected Value of Perfect } \\
\text { Information }\end{array}$} & $\begin{array}{c}\text { Expected Value of Imperfect } \\
\text { Information }\end{array}$ & $\begin{array}{c}\text { Collect } \\
\text { Information? }\end{array}$ \\
\cline { 2 - 6 } & No HLW (p) & TRU (q) & No HLW (p) & TRU (q) & No \\
\hline \hline $241-B-201$ & 0.0 & 0.8 & 0.0 & 0.0 & No \\
\hline $241-B-202$ & 0.0 & 0.6 & 0.0 & 0.0 & No \\
\hline $241-B-203$ & 0.0 & 1.5 & 0.0 & 0.0 & No \\
\hline $241-B-204$ & 0.0 & 1.5 & 0.0 & 0.0 & No \\
\hline $241-T-201$ & 0.0 & 0.8 & 0.0 & 0.0 & No \\
\hline $241-T-202$ & 0.1 & 0.5 & 0.0 & 0.0 & No \\
\hline $241-T-203$ & 0.0 & 1.0 & 0.0 & 0.0 & No \\
\hline $241-T-204$ & 0.0 & 1.1 & 0.0 & 0.0 & Yes \\
\hline $241-A W-13$ & 0.0 & 5.8 & 0.0 & 2.2 & Yes \\
\hline $241-A W-104$ & 0.0 & 4.4 & 0.0 & 0.8 & Yes \\
\hline $241-T-110$ & 4.0 & 23.1 & 0.0 & 17.7 & Yes \\
\hline $241-T-111$ & 4.0 & 23.0 & 0.0 & 17.0 & No \\
\hline $241-B-110$ & 0.0 & 3.4 & 0.0 & 0.0 & No \\
\hline $241-B-111$ & 0.0 & 3.2 & 0.0 & 0.0 & No \\
\hline $241-B-112$ & 0.0 & 0.0 & 0.0 & 0.0 & \\
\hline \hline
\end{tabular}


The calculation of the EVSI was based on the diagnosticity matrixes similar to the ones shown in Table 4.19. Interestingly, the EVSI is zero for historical information, because it has a fairly low diagnosticity. The EVSI for sampling information about TRU is also zero, except for four tanks. For Tanks 241-AW-103 and 241-AW-105, the EVSI is just high enough to justify taking one or two core samples. For Tanks 241-T-110 and 241-T-111, the EVSI is substantial and would justify taking several samples.

The expected value of perfect information about the mass of vitrified waste (influenced by loading factors) was between $\$ 0.0$ million and $\$ 5.6$ million for the low-volume tanks and 241-B-110 and 241-B-111. The EVPI for Tanks 241-AW-103 (EVPI=\$13.3 million), 241-AW-105 (EVPI=\$8.6 million), 241-T-110 (EVPI $=\$ 28.2$ million), and 241-T-111 (EVPI =32.3 million) was substantial. Similarly, the EVPI for the cost of processing a MT of waste is fairly high. The reason information on mass and cost has such a large impact is that, in some cases (low mass of TRU and low cost of TRU), it would be preferable to process and dispose TRU wastes as TRU wastes, while in others (high mass of TRU and high cost of TRU), it would be preferable to process TRU wastes as HLW. Thus, depending on the resolution of mass and cost uncertainties, the decision for TRU wastes will switch.

These VOI calculations suggest the following strategy for collecting waste information from the 15 potential TRU tanks:

1. Obtain better information on the relative costs of processing wastes as TRU or HLW. This involves getting better estimates of the wastes that need to be vitrified, the loading factors, and the cost of processing and disposing one MT of waste. Initially this should be done by a review of the tank waste inventories and cost estimates. If this is not sufficient to resolve the issue of whether TRU wastes or HLW are cheaper to process and dispose of, additional information sources should be examined. How much to pay for these sources can easily be determined by re-running the current analysis with the updated probability distributions for mass, loading factors, and cost.

2. Do not collect any more information about whether any of the 15 potential TRU tanks have been contaminated with HLW. Possible exceptions to this suggestion are Tanks 241-T-110 and 241-T-111, for which at least the EVPI for this variable is $\$ 4$ million (even though the expected value of imperfect information is zero for both cases). If a more reliable historical analysis could be designed that would come close to resolving the issue of whether these tanks have been contaminated with HLW, it should be conducted.

3. Collect core samples for Tanks $241-T-110$ and $241-T-111$ to determine the TRU activity level. If the samples indicate that the wastes are TRU, process and dispose of them as TRU (or as HLW, if this turns out to be cheaper). If the samples indicate that the wastes are LLW, process and dispose of them as LLW. 
4. Possibly collect core samples for Tanks 241-AW-103 and 241-AW-105. If the sample indicate that the tank wastes are TRU, process and dispose of them as TRU, if this is cheaper than processing and disposing of them as HLW.

5. For all other tanks, process and dispose of the wastes as HLW.

\subsubsection{VOI Model for Technetium Feed Specification Decision}

\subsubsection{Technetium Characterization Problem}

The technetium (99 $\mathrm{Tc}$ ) characterization problem is mapped schematically in Figure 4.15. DOE's objective is to set a LLW feed envelope and a product specification for ${ }^{99} \mathrm{Tc}$ (represented by rectangles) that minimize total cost and schedule, including losses due to failure to meet these specifications. The total quantity of ${ }^{99} \mathrm{Tc}$ in the LLW fraction of tank waste, represented as a circle on the left of the figure, is a key unknown variable in this decision problem. Based on DOE's feed envelope and product specifications, the vendor must make engineering and design decisions and accept certain risks inherent in the overall uncertainty of the problem, including those associated with the performance characteristics of the technologies selected. These decisions and risks are represented by the schematic decision tree enclosed by rectangles. Following from those vendor decisions will be results in the form of project cost, schedule, and performance.

The feed envelope and product specification decisions are made based on knowledge about the total quantity of ${ }^{99} \mathrm{Tc}$ in the LLW, the direct costs of setting these specifications, and potential losses that may be incurred if the feed or product does not meet the specifications for ${ }^{99} \mathrm{Tc}$. The analysis approach we use presumes that the costs and losses are a function of the estimated quantity of ${ }^{99} \mathrm{Tc}$ and the actual quantity of ${ }^{99} \mathrm{Tc}$. This loss function approach will suggest how much ${ }^{99} \mathrm{Tc}$ characterization information should be purchased, based on the diagnosticity of that information and the cost of gathering it.

Characterization information can provide an imperfect indication of the unknown ${ }^{99} \mathrm{TC}$ quantity before the feed envelope and product specification decisions must be made. This characterization information is imperfect in that it is probabilistically dependent on the actual quantity of ${ }^{99} \mathrm{Tc}$; perfect knowledge about the quantity of ${ }^{99} \mathrm{Tc}$ is not possible. Current tank characterization information about ${ }^{99} \mathrm{Tc}$ quantities can be used to suggest a feed envelope and a product specification for ${ }^{99} \mathrm{Tc}$ that minimize expected costs and losses from not meeting specifications. The decrease in expected costs and losses associated with additional characterization information can be compared with the cost of obtaining that additional information. In addition, different characterization strategies can be compared with one another in terms of their expected decrease in expected costs and losses. 


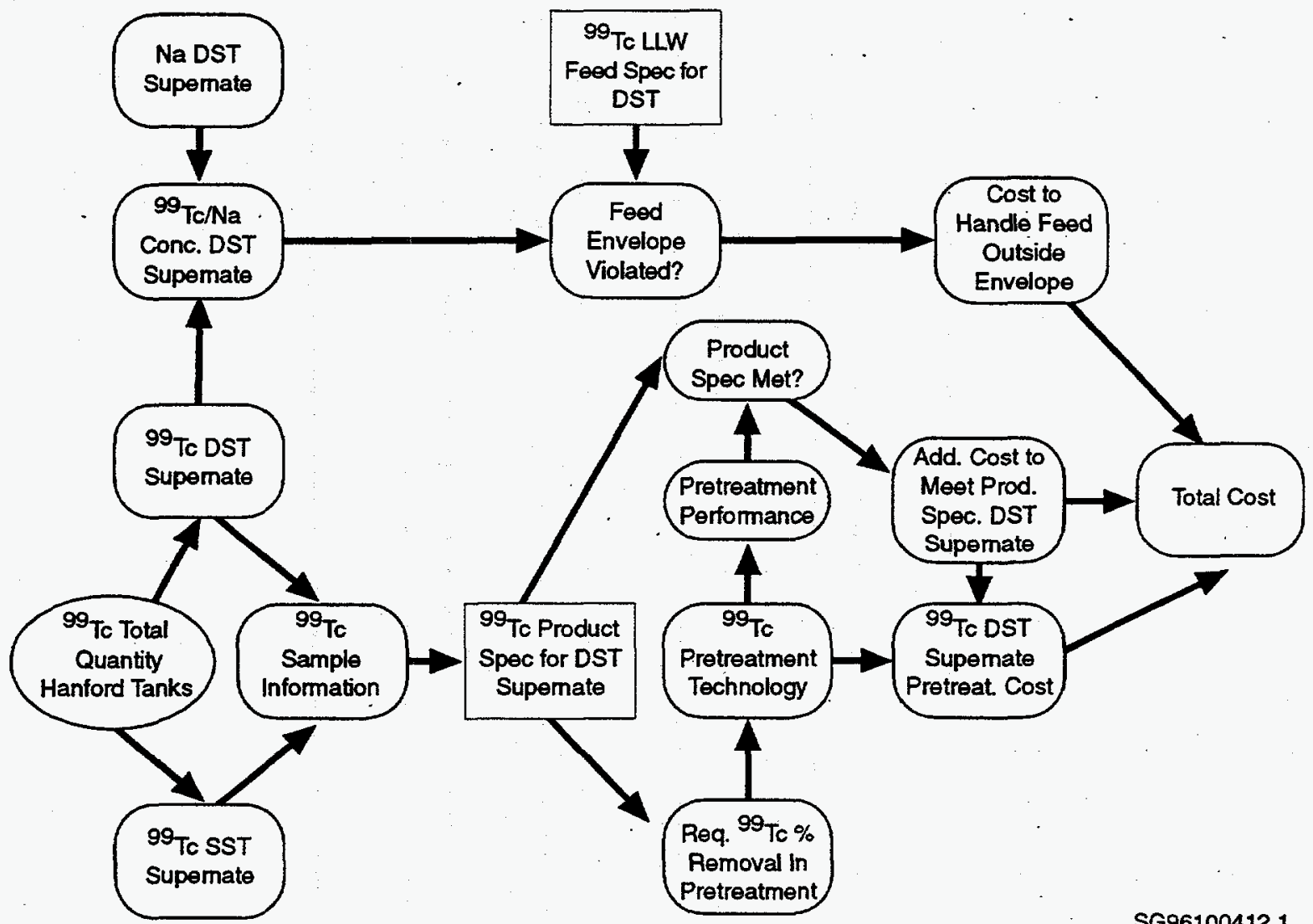

Figure 4.15. Influence Diagram for ${ }^{99} \mathrm{Tc}$ Characterization

\subsubsection{Methodology Overview}

The following steps were followed for evaluating the expected VOI regarding the total quantity of ${ }^{99} \mathrm{Tc}$ in the LLW fraction of tank waste:

1. A prior probability distribution was determined for the total quantity of Tc in all SST and DST supernate to be processed as LLW and disposed onsite. This prior distribution took the form of a normal distribution (Section 4.2.3.3).

2. The ${ }^{99} \mathrm{Tc}$ disposal problem was examined to develop a loss function that captures the lifecycle cost and waste volume consequences suggested in the decision representation of Figure 4.10 (Section 4.2.3.4). While this loss function is necessarily an approximation of the decision outcomes (consequences), it is constructed to capture the essential properties of the decision problem. The problem was investigated from the vendors' perspective to gain a better understanding of how Tc quantities might drive LLW pretreatment options. The loss function took the general form of a piece-wise quadratic (or linear) 
loss, where greater deviations between the actual total quantity of ${ }^{99} \mathrm{Tc}$ and that assumed for setting a product specification resulted in greater losses. The increase in loss was either quadratic (base case) or linear. Over-estimates were penalized more than under-estimates by an optimists' penalty factor of 2 to 20 times that for the pessimist (base case of 5 times).

3. An optimal estimate of the actual quantity of ${ }^{99} \mathrm{Tc}$ was determined (called $\mathrm{m}^{*}$ ) that minimized the expected loss using the assessed prior probability distribution for the mean, $\mu$. This calculation was performed analytically using an integration approximation algorithm.

4. Several possible information sources were identified (including tank sampling) that could result in new information that would suggest certain modifications of the prior distribution over $\mu$. Rather than model the diagnosticity of each of these information sources in detail, a family of (normal) likelihood functions is suggested. These likelihood functions are parameterized to reflect a broad range of different information sources; each requires that the information source be related to an observed quantity of ${ }^{99} \mathrm{Tc}$ over the entire LLW inventory. Each of these sources is considered to be equivalent to producing a "random sample" of ${ }^{99} \mathrm{Tc}$ quantity ( $\mathrm{kCi}$ ) from the entire $\mathrm{LLW}$ inventory, with known variance. The process is assumed to be normally distributed around the actual (unknown) ${ }^{99} \mathrm{Tc}$ quantity, and the variance is parameterized to explore a range of information sources, representative of various levels of diagnosticity. Using Bayes Theorem and the integration approximation algorithms, the posterior distributions for $\mu$ were calculated.

5. Using these posteriors, expected losses were recalculated, substituting $m^{*}(x)$ for $m^{*}$ and taking the expectation over information outcomes, $x$. The difference in the expected loss with information and the expected loss without information from step (3) is reported as the expected value of the imperfect (sample) information (Section 4.2.3.8).

6. Various sensitivity analyses were conducted on the expected value of information as a function of the parameterized loss functions and the diagnosticity of the information source (Section 4.2.3.10). The steps are described in detail in the following sections.

\subsubsection{Prior Distribution}

For this report, a normal prior distribution for the total quantity of ${ }^{99} \mathrm{Tc}$ (expressed in $\mathrm{kCi}$ ) present in SST and DST supernate was used. The prior distribution follows the analysis by Colby and Petersen (1995) and is normal with a mean of $22.27 \mathrm{kCi}$ and a standard deviation of $3.74 \mathrm{kCi}$. As shown in Figure 4.16, this prior distribution is diffuse and covers the range of plausible estimates of ${ }^{99} \mathrm{Tc}$ in tank supernate. Although not technically bounded, the normal prior assigns probability density of some practical significance to ${ }^{99} \mathrm{Tc}$ quantities between $11,000 \mathrm{Ci}$ (about $650 \mathrm{~kg}$ ) to $34,000 \mathrm{Ci}$ (about $2000 \mathrm{~kg}$ ). 


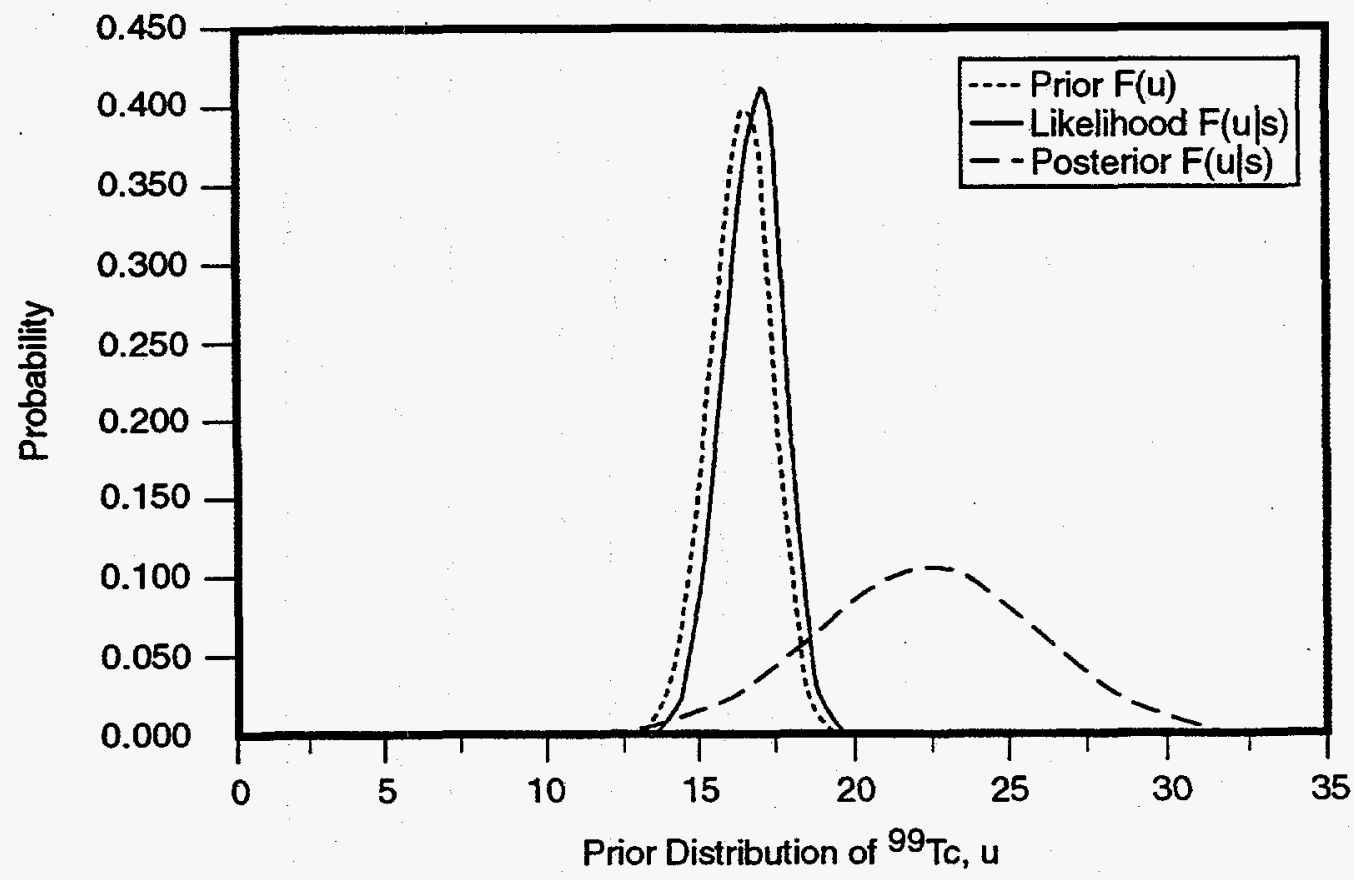

SG96100412.9

Figure 4.16. Prior Distribution of ${ }^{99} \mathrm{Tc}, \mu$

\subsubsection{Description of Loss Function}

The following general form of the loss function (L) was chosen:

$$
\begin{array}{lll}
L(u, D)= & r(D-u)^{c} & \text { for } D>u \\
k r(u-D)^{c} & \text { for } D<u \\
0 & D=u
\end{array}
$$

where $\quad \mathbf{u}=$ is the actual value of ${ }^{99} \mathrm{Tc}$

$\mathrm{D}=$ is the estimate of ${ }^{99} \mathrm{Tc}$

$r=$ is a proportionality constant for calibrating the loss function $(r>0)$

$\mathbf{k}=$ is a multiplier penalty for optimistic estimates (D) less than the true quantity $(u)(k>0)$

c. $=$ is an escalation coefficient.

The escalation coefficient represents the case in which higher costs are proportional to the difference between $u$ and $D(c=1)$ or an extreme case in which significantly higher costs are incurred only when the difference between $u$ and $D$ are greater than a particular threshold $(c>1)$.

For $k=1$ and $c=2, L$ is proportional to squared error loss; for $k=1$ and $c=1, L$ is proportional to absolute error loss. Since underestimates are assumed to be always worse than overestimates of the same magnitude, it was assumed that $k>1$ always. Figure 4.17 plots the linear $(c=1)$ version of $L$ for 


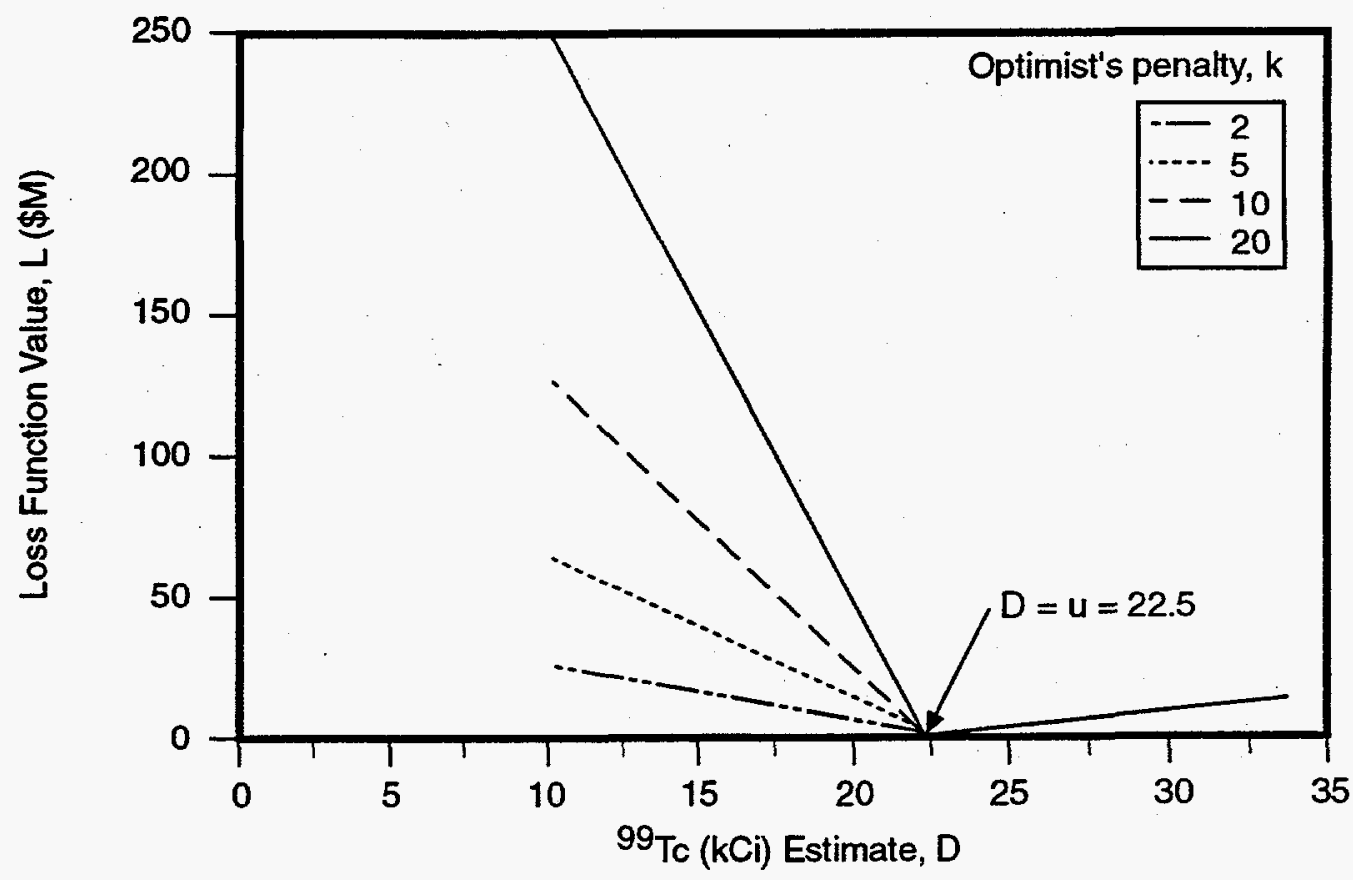

SG96100412.10

Figure 4.17. Linear Loss Functions, $L(u, D)$ Where $u=22.5$ ) for Varying Values of the Optimist's Penalty, k

four values of $k(2,5,10,20)$; Figure 4.18 plots the quadratic $(c=2)$ version of $L$ for the same four values of $k$ and $r=0.1$. In both cases, the plots are for the case of $u=22.5$, and the $x$-axis represents different estimates (D) of the true quantity of ${ }^{99} \mathrm{Tc}$. In all cases, when $\mathrm{D}=22.5$, the loss $(\mathrm{L})$ is 0 , since the estimate is equal to the true value of the ${ }^{99} \mathrm{Tc}$ quantity. Note that in both cases, there is an entire family of curves generated for different values of $u$, the random true quantity of ${ }^{99} \mathrm{Tc}$.

\subsubsection{A Specific Loss Function Ilustration}

The general form of the loss function (L) was selected to capture the increase in disposal costs that arise from setting a ${ }^{99} \mathrm{Tc}$ product specification based on an [incorrect] estimate (D) of the true quantity (u) of ${ }^{97} \mathrm{Tc}$ in the SST and DST supernate. The loss function $(\mathrm{L}[\mathrm{u}, \mathrm{D}])$ is sensitive only to deviations between the estimate (D) and the actual quantity (u) of ${ }^{99} \mathrm{Tc}$, and not to their absolute value. Thus, the fact that the overall cost of disposal is increased with higher values of $u$ and $D$, even when they are consistent, is not captured by the loss function. For all variations, when $u=D$, the loss is fixed at 0.0 . Although the absolute value of losses has not been exactly calibrated, the values of $\mathrm{L}$ roughly correspond to additional lifecycle costs measured in millions of dollars. 


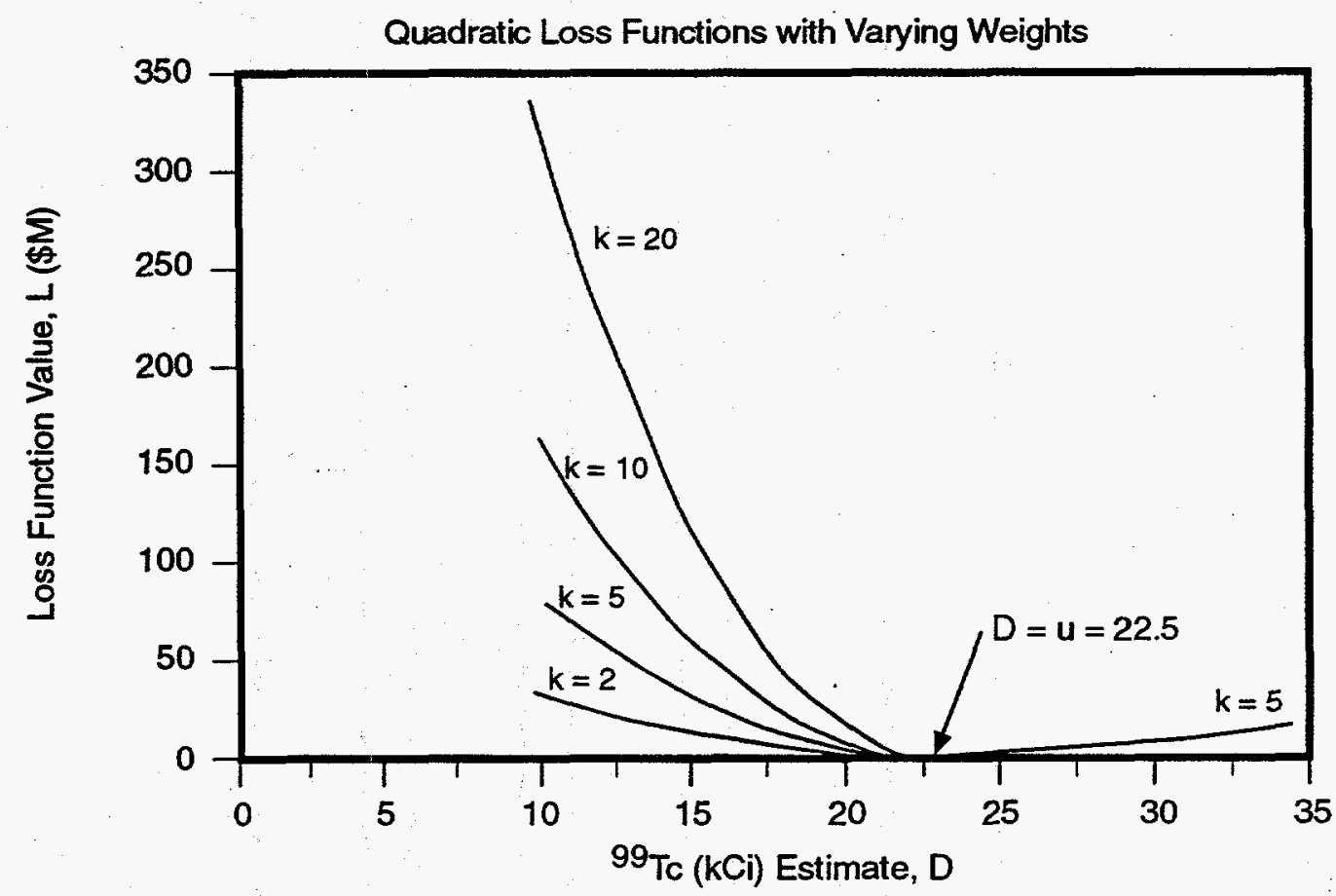

SG96100412.11

Figure 4.18. Quadratic Loss Functions, $L(u, D)$ Where $u=22.5$ ) for Varying Values of Optimist's Penalty, k

The following loss function was selected to capture the incremental costs from misestimation of the total quantity of ${ }^{99} \mathrm{Tc}$ :

$$
\begin{array}{ll}
\mathrm{L}(\mathrm{u}, \mathrm{D})=0.1(\mathrm{D}-\mathrm{u})^{2} & \text { for } \mathrm{D}>\mathrm{u} \text { (overestimation) } \\
0.5(\mathrm{u}-\mathrm{D})^{2} & \text { for } \mathrm{D}<\mathrm{u} \text { (underestimation) } \\
0 & \text { for } \mathrm{D}=\mathrm{u} .
\end{array}
$$

Loss (L) is plotted as a function of the estimate (D) in Figure 4.17 for $u=22.5$ assuming a linear loss and in Figure 4.18 for $\mathrm{u}=22.5$ assuming a quadratic loss. Note that the loss function captures the characteristic of negligible losses when $\mathrm{D}$ is close to $\mathrm{u}$, accelerating as $\mathrm{D}$ moves away from $\mathrm{u}$. The function also captures the greater potential for large incremental costs when $u$ is underestimated than when $u$ is overestimated.

\subsubsection{Optimal Estimate Based on Prior Probability (no further characterization information)}

The plots in Figures 4.17 and 4.18 are conditional on particular values of $u$, which is the uncertain parameter for which additional information is sought. But how good is the estimate with no additional 
information? One way to gauge our state of knowledge (based on the prior probability of ${ }^{99} \mathrm{Tc}$ ) is to calculate the expected loss for each possible value of the ${ }^{99} \mathrm{Tc}$ estimate (D), where the expectation is taken with respect to the prior probability distribution over the actual value, $u$. The "best estimate" is the one that yields a minimum expected loss with respect to the prior probability distribution, unenlightened by additional characterization information. Figure 4.19 shows these expected losses as a function of the estimates (D) for the linear loss function specified.

Figure 4.20 shows these expected losses as a function of the estimate (D) for the quadratic loss function specified.

The asymmetry of $\mathrm{L}$ suggests that the ${ }^{99} \mathrm{Tc}$ best estimate is greater than the mean of the prior, $22.27 \mathrm{kCi}$. The minimum expected loss comes at $\mathrm{D}=24.5 \mathrm{kCi}$ (Figure 4.20 ). Thus, the best estimate is only about $60 \%$ of a standard deviation above the mean of the prior.

\subsubsection{Likelihood Function}

As described above, all possible information sources have not been modeled in detail. Instead, various information sources are represented as comparable (or equivalent) to a sample estimate of the tank waste with a particular diagnosticity. The sample is assumed to be distributed normally about the actual parameter

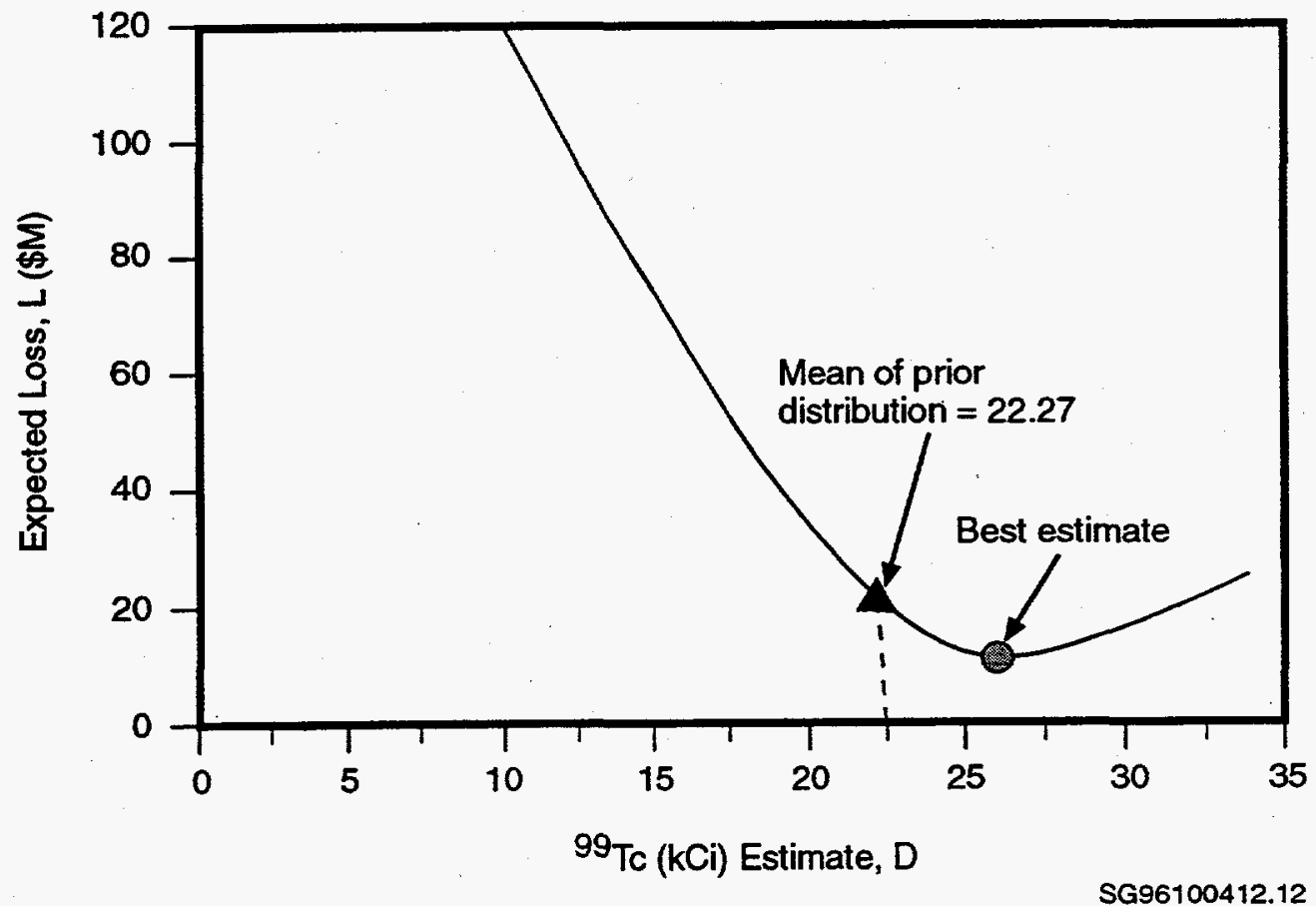

Figure 4.19. Expected (linear function, $k=5$ ) Loss as a Function of the ${ }^{99} \mathrm{Tc}$ Estimate 


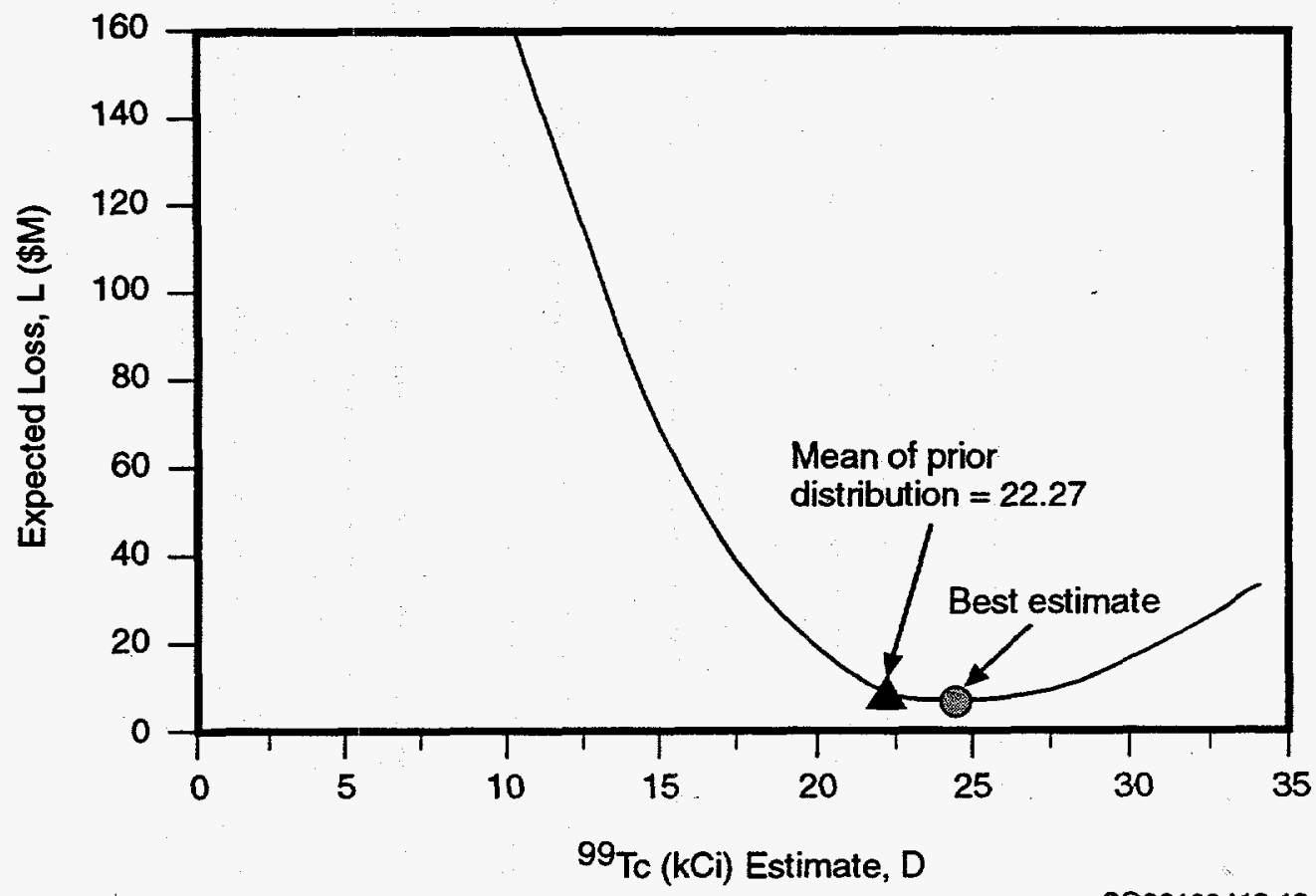

Figure 4.20. Expected (quadratic function, $k=5$ ) Loss as a Function of the ${ }^{99} \mathrm{Tc}$ Estimate

value for $u$, with a standard deviation that matches the diagnosticity of the information. For purposes of this report, four distinct values of diagnosticity are considered $(s=200 \mathrm{Ci}, 500 \mathrm{Ci}, 1000 \mathrm{Ci}$, and $2000 \mathrm{Ci}$ ). These values (s) represent the standard error of a sample about its mean, $u$. Thus, $x$ is assumed to be normally distributed, with mean $u$ and standard deviation $s$, depending on the information source. An example of a likelihood function for the case $u=16,000 \mathrm{Ci}$ and $\mathrm{s}=1000 \mathrm{Ci}$ is plotted in Figure 4.16 (left-most peaked curve). Further work is needed to map various information-gathering activities (including, but not limited to, sampling tanks) into values of $\mathbf{s}$.

\subsubsection{Posterior Distributions}

As outlined in the overview, the likelihood functions can be combined with the prior distributions and a posterior distribution computed for $u$, conditional on any observed value of $x$ (the sample mean or "mean equivalent"). Continuing with the example above, Figure 4.16 also displays an example of a posterior distribution for $u$ given observation of $x=16$. Note that while the posterior distribution is peaked near $u=16$, the expected value of the posterior is greater than 16 , and is in fact a weighted average of the mean of the prior and the sample mean.

These posterior distributions are very useful for calculating expected losses given observation of a particular sample mean. Figure 4.21, for example, plots values of expected quadratic loss $(c=2, k=5)$ 


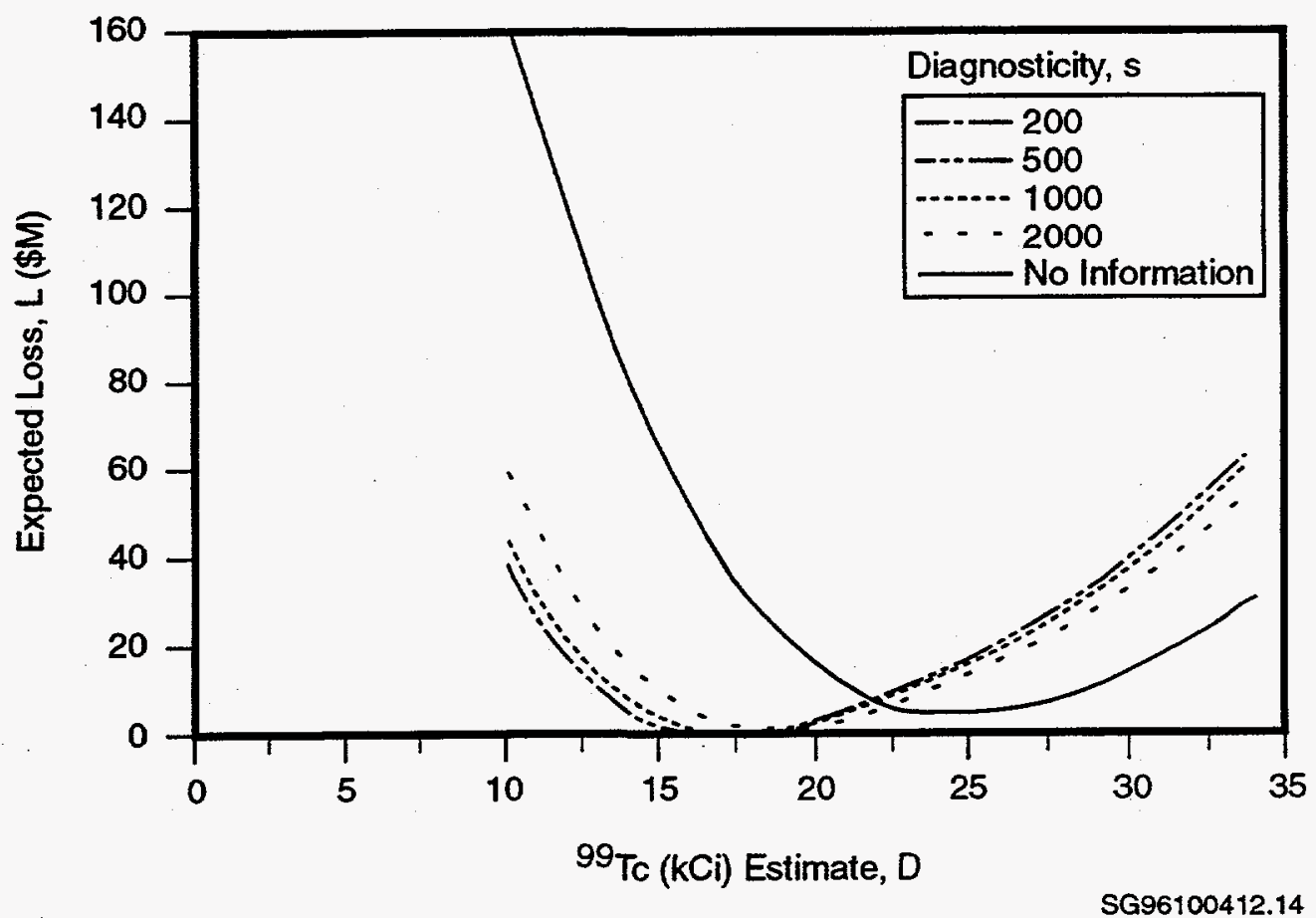

Figure 4.21. Expected Loss (quadratic, $k=5$ ) for Different Levels of Diagnosticity $(s=200,500,1000$, and $2000 \mathrm{Ci}$ ) Compared with the No Information Case

given an observation of $x=16$. The different curves correspond to different values of $s(200,500,1000$, $2000 \mathrm{Ci}$ ) in the likelihood function. For comparison, the expected loss curve with respect to the prior (no additional information) is also plotted. Each point corresponds to the expected loss with respect to the posterior (or prior) for a particular estimate (D) of ${ }^{99} \mathrm{Tc}$ in the supernate. While 24.5 is the optimal estimate with no information, it can be seen that sample information moves the optimal estimate closer to the mean equivalent estimate. The best estimate is much closer to the sample when the sample is of greater diagnosticity ( $s=200$ or $500 \mathrm{Ci}$ ), but even sample equivalents with diagnosticity comparable to the $s=1000$ or $2000 \mathrm{Ci}$ case substantially decrease the expected loss. Even the $s=2000$ case yields an expected loss that is much closer to perfect information $(E(L)=0$ ) than to the no information case using only the prior distribution. Deciding whether this decrease in expected loss is worth the cost of the samples will require further calibration of the loss function.

\subsubsection{Sensitivity of Loss Function}

The two forms of loss function analyzed in this illustration are the linear function (Figure 4.17) and the quadratic function (Figure 4.18). Other forms for loss functions can be analyzed. Sensitivity analysis was performed on $L$ varying the optimistic multiplier penalty $(k)$ over four values $(2,5,10,20)$ and the escalation coefficient $(c)$ over the linear $(c=1)$ and quadratic $(c=2)$ cases in this illustration. Together, these eight cases cover a broad range of possible loss functions for this problem. Further detailed analysis with more sensitive loss functions or better calibration parameters ( $r$ ) could be performed. 


\subsubsection{Expected Value of Information of Varying Diagnosticity}

Figure 4.21 shows the usefulness of information of varying degrees of accuracy, all conditional on a single observation of $x=16 \mathrm{kCi}$. Figure 4.22 plots values of expected linear loss $(c=1)$ for varying values of the $\mathrm{k}$ weighting parameter. The plots show the expected losses for each of four levels of diagnosticity $(s=200,500,1000$, and $2000 \mathrm{Ci}$ ), as well as the no information case of using only the prior with $\mathrm{s}=3740 \mathrm{Ci}$. Note that the steepest decline in expected loss comes from gaining any information, compared with our current state of prior knowledge. This gain is greater in the case of greater asymmetry $(k=20)$ in the loss function. Figure 4.23 plots the same analysis using a quadratic $(c=2)$ loss function. Note that the decrease in expected loss is almost linearly related to the improvement in the sample diagnosticity. Likewise, asymmetry (as observed by the optimists penalty) has a much smaller effect in the case of a linear loss function compared with the quadratic case.

\subsubsection{Conclusions}

- Obtaining a prior distribution over the uncertain parameter, in this case the amount of ${ }^{99} \mathrm{Tc}$ in SSTs and DSTs, was a useful task. All characterization efforts must begin with the question, What is currently known about the uncertain quantity? It is of vital importance to quantify this uncertainty in the form of prior probability distributions obtained from experts armed with all relevant studies and data currently available.

- The expected decrease in costs and losses from obtaining some additional sample information about the quantity of ${ }^{99} \mathrm{Tc}$ in the LLW appears to justify the cost of gathering this information. Additional effort is needed to map potential information sources with the information diagnosticity index(s) used in the analysis.

- There is some indication that additional efforts to understand more about the likelihood function of highly diagnostic samples are not as useful as efforts spent to further characterize priors representative of all that is known and to develop a valid loss function.

- The loss functions, within the families tested, demonstrated consistent results and minor variations in shape may not be that important. 


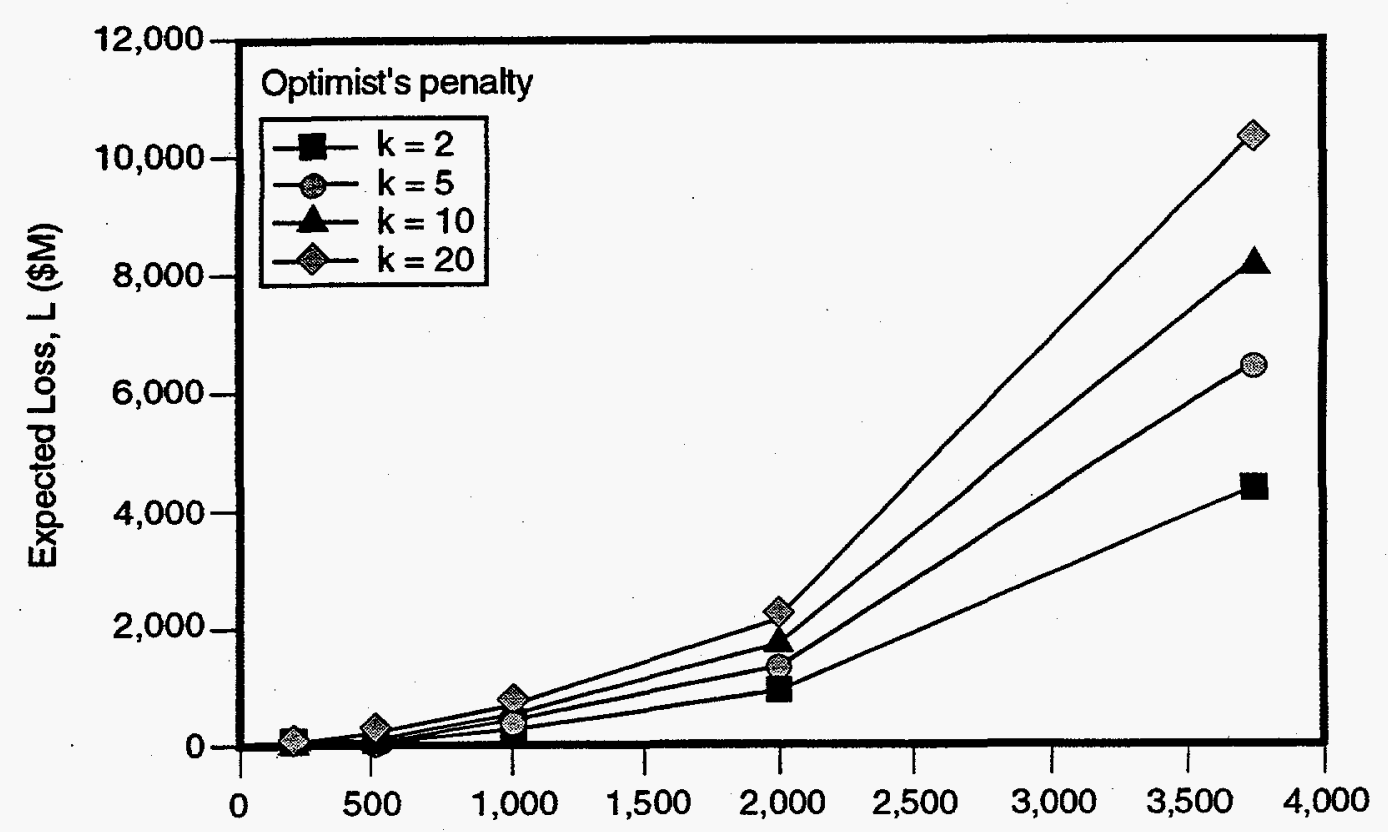

Diagnosticity, $s(\mathrm{Ci})$

SG96100412.15

Figure 4.22. Expected Linear Loss as a Function of Optimists Penalty (k) and Diagnosticity of the Information Source

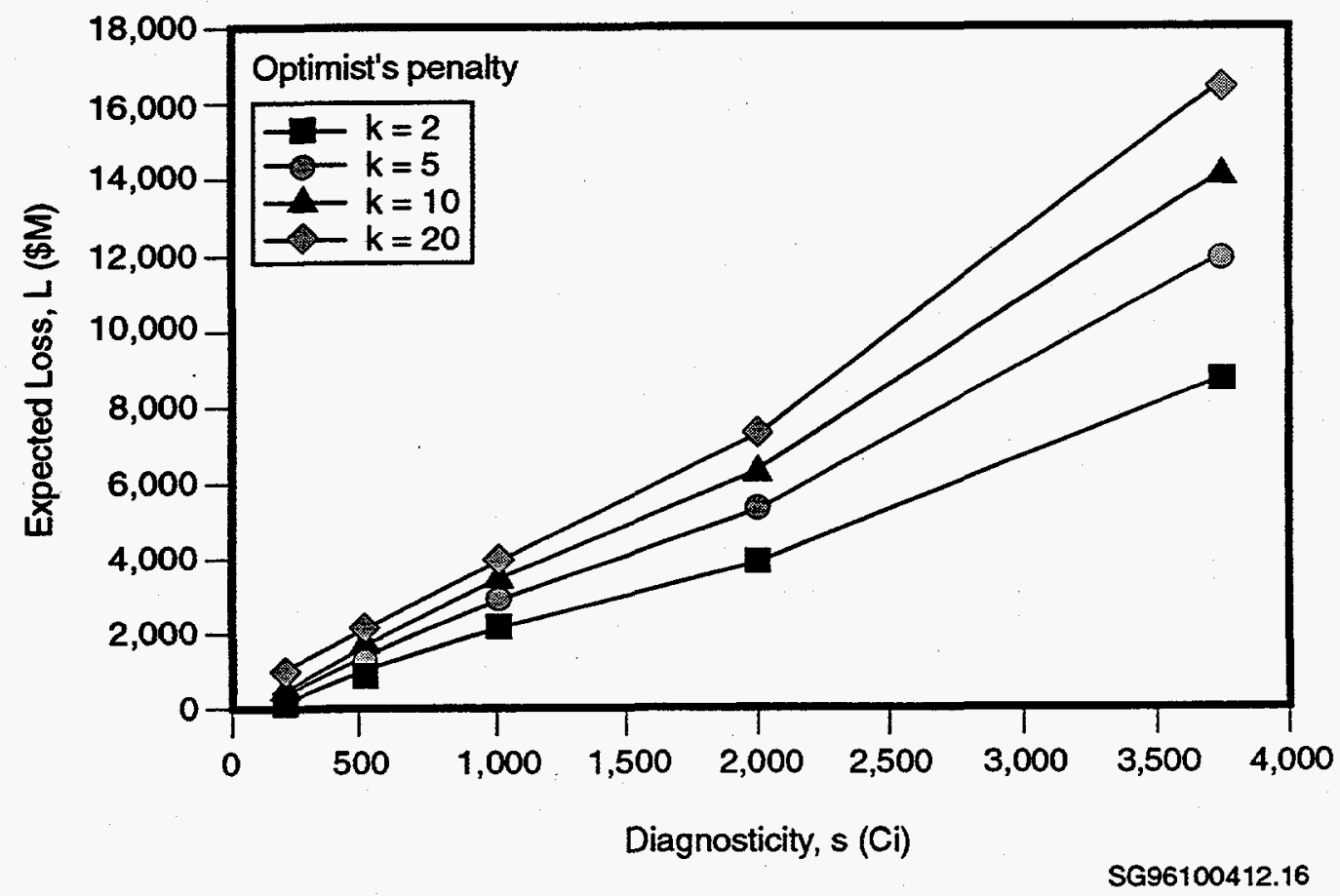

Figure 4.23. Expected Quadratic Loss as a Function of Optimists Penalty (k) and Diagnosticity of the Information Source 



\subsection{Basis for Integration of VOI and Prioritization of Requirements}

Previous sections of this report illustrated how analyses of several tank waste management decisions can lead to quantitative estimates of the VOI for the key measurable parameters in a tank waste safety or disposal problem. This section addresses how these results can be integrated and used to plan a costeffective and operationally specific characterization program.

\subsection{Integration}

A useful point of departure for this section is the observation that the VOI method requires a "welldefined" problem. That is, the decisions and outcomes can be defined, an appropriate measure of value for the problem can be specified, the relevant measurable parameters can be identified, and our degree of certainty or knowledge about each of these can be quantified. Given these conditions, the method will work. However, several important questions remain in guiding the application of this method and integrating its results.

First, how and where should the method be applied? Do all issues requiring some support from characterization need a formal VOI study? Should the method be applied to all tanks for those issues where it is clearly relevant? How should the safety issues be partitioned for purposes of VOI analysis?

Given that VOI analyses can be conducted for a set of discrete issues, how can the results be used to define specific sampling plans and a general characterization strategy? Should these sampling plans be defined at the issue level or aggregated somehow?

These questions are addressed in this section. No attempt has been made to provide detailed answers in terms of a program plan and schedule for implementation, but the answers set forth are specific in the sense of policy recommendations.

\subsubsection{Bases for Integration}

If information were free, we would not need to worry about its value. In the tank waste context, there are substantial dollar and schedule costs associated with characterization activities. Obtaining better estimates of relevant inventories and behavioral parameters is so costly it gives us the incentive to carefully estimate the VOI, which enables us to determine the appropriate expenditure of resources on acquiring information.

Thus, in simple terms, if we can estimate the VOI for some bit of information, we can compare it with the cost of obtaining the information and make a rational decision about whether to obtain it. If the VOI measure is a monetary one, this is straightforward. If the expected information from a proposed sampling and analysis scheme would decrease the expected waste treatment cost by $\$ 6.0$ million, we would spend up to that much to implement it (given that schedule and other factors were equal). In practice, we end up by expressing VOI as a function of the extent (number of samples) or the degree of accuracy of individual 
assays or sets of assays. When VOI is defined this way, the cost-effectiveness criterion becomes one of defining the sampling program that produces the highest net benefit (i.e., excess of VOI over cost of sampling and analysis). Analysis includes whatever statistical, physical-chemical, and other science is needed to extract best estimates of the parameters from laboratory data. Since VOI will often show "decreasing (marginal) returns" as a function of sample size or accuracy, this becomes equivalent to finding the extent of sampling or accuracy enhancement at which the incremental VOI is just equal to the unit sampling and analysis cost.

\subsubsection{Measuring Values on a Dollar Basis}

What about cases in which the value in VOI is not expressed in monetary terms? We have measured the VOI in terms of the reduction of the statistical expectation of some dose or other consequence measure. How can this VOI estimate be used? Estimating value required that quantitative tradeoffs be made among different consequences, e.g., dose (in REMs) and costs (in dollars). These tradeoffs allow aggregation of difference type of consequences into a single measure of value (typically dollars).

\subsection{Integrating Value of Information to Create a Characterization Strategy}

Various types of integration are needed to create a characterization strategy. This integration is discussed below, followed by a description of the specific steps needed to create the strategy and a discussion of some technical issues associated with aggregating VOI.

\subsubsection{Types of Integration}

As VOI analyses are performed for numerous tank waste decisions, the results of individual analyses must be integrated to create an overall strategy. Three types of integration may be needed:

- across sources of information, e.g., multiple samples or sampling and modeling

- across tanks for a specific decision, e.g., the organic nitrates and TRU decisions discussed previously

- across decisions, e.g., if safety mitigation actions affect disposal options.

Integration across tanks is perhaps the simplest problem. Two linkages across tanks are possible. First, information collected for one tank will update the prior information about other tanks, especially if they have similar contents. A process should be in place to update (through re-elicitation of experts, if necessary) the probability distributions over tank waste variables, after new information about one tank becomes available. Second, similar or identical decisions are linked across tanks. For example, whether to keep TRU separate from HLW is a TWRS-wide decision, but it could be made on a tank-by-tank basis. Of course, this approach works only if the application of a good decision rule for each tank separately also results in a good decision rule for all tanks. 
Integration across sources of information is more complex. First, information from one source may change the likelihood functions for other information sources. As with the updating of priors across tanks, likelihood functions should be updated across information sources. Second, the same information is often relevant for many different decision problems. When the decision problems are separable (i.e., the consequences in each problem depend only on the alternatives in the problem, not on the combination of the alternatives across problems), aggregation is relatively straightforward and adding VOIs is appropriate. Third, information usually comes in batches. For example, when taking a core sample for a safety decision, analyzing for constituents affecting disposal decisions does not cost much more. Thus, the core sample would contain multiple pieces of information that can be relevant to multiple decisions. If the decision problems are separable, the overall value of the different pieces of information can be assessed by adding VOIs across decisions.

Integration across interlinked decisions is the most complex task. For example, if the mitigation alternatives for a safety decision influence the consequences of a disposal decision, the proper VOI analysis would have to examine the combined decision situation. On the other hand, if the decisions are separable, the separate VOI analyses could be used to create an overall strategy. The VOI analyses would determine the information to be collected. The timing of the decisions would determine the sequence in which the information is collected.

\subsubsection{Basic Approach to Aggregation and Prioritization}

A general approach to aggregation of VOI and creating a characterization strategy consists of the following:

1. For each decision, determine from the VOI analysis what characterization information gathering activities are justified (its value exceeds its acquisition costs) for the individual decision.

2. For each of these information gathering activities, do a rough aggregation (qualitatively considering factors affecting additivity as discussed in Section 5.2.3) of value over decisions and estimate the aggregate cost of the information gathering.

3. Identify remaining information-gathering activities with some value for multiple decisions, but not enough from any single decision to justify the cost of collection. For these activities, do a rough (qualitative) aggregation of these VOIs across decisions (considering factors affecting additivity as discussed in Section 5.2.3) and aggregate costs (considering efficiencies) to determine if information collection is justified.

4. For all activities identified in 2 and 3 above, prioritize based on the ratio of VOI to cost. This prioritization will be somewhat subjective because of the need in 2 and 3 to qualitatively consider additivity. Nevertheless, it should be a reasonable first cut and will point out specific areas where more explicit quantitative aggregation will be useful. 
5. Plan characterization from this prioritized list, including activities with top priority down, until available funding is used.

\subsubsection{Additivity Issues and Approaches}

The value of the joint information is not necessarily equal to the sum of individual information values. When it is not, there is an easily understood rationale for this nonadditivity. An example in which information values have been shown to be non-additive for tank characterization involves the value of organic fuel and moisture information on a tank. In this instance, the values of fuel and moisture information together may be less than the sum of their values considered independently. Intuitively, what this says is that its not worth assessing fuel very carefully for tanks in which the waste is known to be wet, a sensible result. In this type of situation, the information values are "sub-additive."

Another example illustrates that information values may also be "super-additive." If the two estimates involved are for the organic fuel (TOC or energy measure) concentration in a given region of waste and the threshold at which a reaction will propagate in such waste, the value of both types of information jointly will exceed their individually calculated VOIs because it is the combination of these two estimates that really matters for the phenomenology in question.

There are several other plausible cases in which information values for tank waste information may be non-additive. A few are described to illustrate the possibilities. Tactics for dealing with some of these cases are mentioned.

Any time tanks share a common set of conditions or phenomenology, there is likely to be nonadditivity in the sense that information on the waste in one tank can be exploited for making estimates about the waste in others. This type of non-additivity can be quantitatively assessed in the context of the tank grouping schemes that are used for making best estimates of inventories and other parameters.

When there are "overriding issues" for specific tanks, information values are likely to be non-additive. This situation might occur when resolution of one safety issue required some immediate direct action which rendered another issue a moot point. This type of situation is quite likely to arise in characterizing waste as fit for treatment, when one or two constituents will typically be rate-limiting. There may also be non-additivity in information values when two safety issues are phenomenologically related. Thus the value of both headspace gas sampling for the flammable gas issue and condensed phase sampling for the organic issue may be less than the sum of their values independently calculated, since we are, in effect, learning about the same set of reactions under different conditions.

It is also possible that the engineering solutions to the various safety or disposal issues are technically. incompatible, which would clearly invalidate the associated VOI estimates. An example (plausible, but not demonstrated) might be that ventilation to ensure flammable gas safety also tends to decrease surface moisture levels and increase risks for condensed phase reactions. The converse is also plausible-adding moisture to the waste surface to decrease the chance of a condensed phase reaction could act to retard the gradual release of flammable gas and increase the hazard from this issue. 
There is expected to be marked super-additivity over different types of spatial variability information and the estimation of extreme values for certain constituents. In general, this involves an estimate of bulk conditions and a spatial model of the waste. This situation arises in both organic condensed phase reaction issues and the criticality issue, where a relatively small volume of waste of the requisite characteristics can pose a problem. In this situation, the VOI from waste samples is strongly conditioned by the validity of the spatial model. Without a good spatial model, we cannot meaningfully interpret such samples in terms of implications for extreme values. Thus the combined information on spatial variability and bulk inventories is probably worth more than the sum of the separate VOIs.

\subsection{Bounding Estimates for VOI}

Since assessing VOI and aggregating over problem domains can be somewhat tedious, and since the characterization program needs some overall guidance on the extent of sampling required as soon as possible, a potential strategy to implement the VOI model is to do some simple bounding estimates for VOI for each issue as the first step. Such estimates would allow some degree of comfort that the stakes were at least roughly commensurate with the cost of sampling. Estimates of this type can be made based on the principle that VOI will never exceed the cost of an effective engineering solution to a problem. In the case of the organic-nitrate reaction, this direct solution might be maintaining sufficient moisture at the waste surface. For the flammable gas issue, it is probably ventilation of headspace. For disposal issues in general, it is designing a process/plant to handle the worst-case waste rather than selectively sequencing and blending waste for treatment.

Such bounding estimates for the value of perfect information about waste constituents and behavioral parameters can be done largely based on engineering work already developed for the safety and disposal programs. This should be the first step in implementing the VOI approach. 



\subsection{References}

Agnew SF, J Boyer, RA Corbin, TB Duran, JR FitzPatrick, KA Jurgensen, TP Ortiz, and BL Young. 1996. Hanford Tank Chemical and Radionuclide Inventories: HDW Model Rev. 3. LA-UR-96-858, Los Alamos National Laboratory, Los Alamos, New Mexico.

Allemann RT, ZI Antoniak, JR Friley, CE Haines, LM Liljegren, and S Somasundaram. 1991. Collection and Analysis of Existing Data for Waste Tank Mechanistic Analysis. PNL-7658, Pacific Northwest Laboratory, Richland, Washington.

Babad H, GD Johnson, DA Reynolds, and DM Strachan. 1992. Understanding of Cyclic Venting Phenomena in Hanford Site High-Level Waste Tanks: The Evaluation of Tank 241-SY-101. WHC-SA-1364-FP, Westinghouse Hanford Company, Richland, Washington.

Babad H, DM Camaioni, MA Lilga, WD Samuels, and DM Strachan. 1993. Tank Chemistry - A New Understanding of Waste Aging. PNL-SA-23183, Pacific Northwest Laboratory, Richland, Washington.

Babad H and DA Turner. 1993. Interim Criteria for Organic Watch List Tanks at the Hanford Site. WHC-SD-WM-ER-189 Rev. 0, Westinghouse Hanford Company, Richland, Washington.

Benar CJ. 1995. Flammable Gas Tank Safety Program: Data Requirements for Core Sample Analysis Developed Through the Data Quality Objectives Process. WHC-SD-WM-DQO-004 Rev 2, Westinghouse Hanford Company, Richland, Washington.

Brevick CH, LA Gaddis, and WW Pickett. 1995a. Historical Tank Content Estimate for Northeast Quadrant of Hanford 200 East Area. WHC-SD-WM-ER-349 Rev 0A, Westinghouse Handford Company, Richland, Washington.

Brevick CH, LA Gaddis, and WW Pickett. 1995b. Historical Tank Content Estimate for Southeast Quadrant of Handford 200 East Area. WHC-SD-WM-ER-350 Rev 0, Westinghouse Handford Company, Richland, Washington.

Brevick CH, LA Gaddis, and ED Johnson. 1995c. Historical Tank Content Estimate for Northwest Quadrant of Handford 200 West Area. WHC-SD-WM-ER-351 Rev 0, Westinghouse Handford Company, Richland, Washington.

Brevick CH, LA Gaddis, and WW Pickett. 1995d. Historical Tank Content Estimate for Southwest Quadrant of Hanford 200 West Area. WHC-SD-WM-ER-352 Rev 0A, Westinghouse Handford Company, Richland, Washington. 
Brewster ME, NB Gallagher, JD Hudson, and CW Stewart. 1995. The Behavior, Quantity, and Location of Undissolved Gas in Tank 241-SY-101. PNL-10681, Pacific Northwest Laboratory, Richland, Washington.

Bryan SA and LR Pederson. 1996. Thermal and Combined Thermal and Radiolytic Reactions Involving Nitrous Oxide, Hydrogen, Nitrogen and Ammonia in Contact with Tank 241-SY-101 Simulated Waste. PNNL 10748, Pacific Northwest National Laboratory, Richland, Washington.

Clemen RT. 1991. Making Hard Decisions. PWS-Kent Publishing Company, Boston.

Colburn RP. 1995. Identification of Potential Transuranic Waste Tanks at the Hanford Site. WHC-SDWM-ES-331 Rev. 0, Westinghouse Hanford Company, Richland, Washington.

Colson SD, RE Gephart, VL Hunter, J Janata, and LG Morgan. 1996. A Risk-Based Focused DecisionManagement Approach for Justifying Characterization of Hanford Tank Wastes. PNNL-11231, Pacific Northwest Laboratory, Richland, Washington.

Cowley WL and DD Stepnewski. 1994. Evaluation of Hazards to Tank Farm Facilities from Lightening Strikes. WHC-SD-WM-SARR-027, Westinghouse Hanford Company, Richland, Washington.

Doherty AL, PG Doctor, AR Felmy, RJ Serne, and AW Prichard. 1996. Characterization Strategy Report for the Criticality Safety Issue (draft). PNNL-11532, Pacific Northwest National Laboratory, Richland, Washington.

Ecology, EPA, and DOE. 1996. Hanford Federal Facility Agreement and Consent Order, as amended. Washington State Department of Ecology, U.S. Environmental Protection Agency, and U.S. Department of Energy, Olympia, Washington.

Goheen SC, JA Campbell, GE Fryxell, LL Burger, RT Hallen, T Jones, GS Klinger, BP McGrail, LR Pederson, RG Riley, and DS Sklarew. 1996. Characterization Strategy Report for the Organics Safety Issue (draft). PNNL-11533, Pacific Northwest National Laboratory, Richland, Washington.

Hanlon BM. 1995. Waste Tank Summary Report for Month Ending August 31, 1995. WHC-EP-0182-89, Westinghouse Hanford Company, Richland, Washington.

Harper BL, SJ Gajewski, LD Muhlestein, CL Glantz, DM Kashporenko, GR Bilyard, AM Nazarali, and AJ Brothers. 1996. Risk Constraint Measures Developed for the Outcome-Based Strategy for Tank Waste Management. PNNL-11302, Pacific Northwest National Laboratory, Richland, Washington.

Hill JG, GS Anderson, and BC Simpson. 1995. The Sort on Radioactive Waste Type Model: A Method to Sort Single-Shell Tanks into Characteristic Groups. PNL-9814 Rev 2, Pacific Northwest Laboratory, Richland, Washington. 
Hodgson KM. 1996. Evaluation of Hanford Tanks for Trapped Gas. WHC- SD-WM-ER-526 Rev. 1, Westinghouse Hanford, Richland, Washington.

Hopkins JD. 1995. Methodology for Flammable Gas Evaluations. WHC-SD-WM-TI-724 Rev 0, Westinghouse Hanford Company, Richland, Washington.

Johnson ME. 1994. Tank Waste Remediation System Decisions and Risk Management. WHC-EP-0786, Westinghouse Hanford Company, Richland, Washington.

Johnson ME. 1996. Tank Waste Remediation System Baseline System Description. WHC-EP-0856, Westinghouse Hanford Company, Richland, Washington.

Keefer D and SE Bodily. 1983. Three-Point Approximations for Continuous Random Variables. Management Science, 29:595-609.

Keeney RL and H Raiffa. 1976. Decisions with Multiple Objectives: Preferences and Value Tradeoffs. Wiley and Sons, New York.

Keeney RL and D von Winterfeldt. 1991. Eliciting Probabilities from Experts in Complex Technical Problems. IEEE Transactions on Engineering Management, 38:191-201.

LANL. 1996. A Safety Assessment of Rotary Mode Core Sampling in Flammable Gas Single Shell Tanks: Hanford, Site, Richland, Washington. WHC-SD-WM-SAD-035 Rev 0, Los Alamos National Laboratory, Los Alamos, New Mexico.

Liebetrau AM, BA Pulsipher, DM Kashporenko, G Chen, F Gao, and SA Hartley. 1996. Uncertainty and Sampling Issues in Tank Characterization (draft). PNNL-11529, Pacific Northwest National Laboratory, Richland, Washington.

McConville CM, ME Johnson, and SL Derby. 1995. Decision Analysis Model for Assessment of Tank Waste Remediation System (TWRS) Waste Treatment Strategies. WHC-EP-0874 Rev. 0, Westinghouse Hanford Company, Richland, Washington.

Merkhofer ML. 1987. "Quantifying Judgmental Uncertainty: Methodology, Experiences, and Insights." IEEE Transactions on Systems, Man, and Cybernetics, Vol. SMC-17, No. 5.

National Research Council. 1994. Building Consensus Through Risk Assessment and Management of the Department of Energy's Environmental Remediation Program. National Research Council, National Academy Press, Washington D.C.

National Research Council. 1996. Barriers to Science: Technical Management of the Department of Energy Environmental Remediation Program. National Research Council Board on Radioactive Waste Management, National Academy Press, Washington D.C. 
Nuclear Regulatory Commission. 1989. Severe Accident Risks: An Assessment of Five U.S. Nuclear Power Plants. NUREG-1150, Nuclear Regulatory Commission, Washington, D.C.

Ortiz NR, TA Wheeler, RJ Breeding, S Hora, MA Meyer, and RL Keeney. 1990. Use of Expert Judgment in NUREG-1150. Nuclear Engineering and Design.

Pulsipher BA, LL Burger, AM Liebetrau, and RD Scheele. 1996. Characterization Report for the Ferrocyanide Safety Issue (draft). PNNL-11530, Pacific Northwest National Laboratory, Richland, Washington.

Raiffa H. 1968. Decision Analysis. Addison-Wesley, Reading, Pennsylvania.

Stewart CW, ME Brewster, and JS Roberts. 1996. Characterization Strategy for the Flammable Gas Safety Issue (draft). PNNL-11531, Pacific Northwest National Laboratory, Richland, Washington.

Strachan DM, WW Schulz, and DA Reynolds. 1993. Hanford Site Organic Waste Tanks: History, Waste Properties, and Scientific Issues. PNL-8473, Pacific Northwest Laboratory, Richland, Washington.

Turner DA. 1993. Corrective Action Strategy for Single-Shell Tanks Containing Organic Chemicals. WHC-EP-0671, Westinghouse Hanford Company, Richland, Washington.

von Winterfeldt D and W Edwards. 1986. Decision Analysis and Behavioral Research. Cambridge University Press, Cambridge, Massachusetts.

Webb AB, JL Stewart, DA Turner, MG Plys, G Malinovic, JM Grigsby, DM Camaioni, PG Heasler, WD Samuels, and JJ Toth. 1995. Preliminary Safety Criteria for Organic Watch List Tanks at the Hanford Site. WHC-SD-WM-SARR-033 Rev. 0, Westinghouse Hanford Company, Richland, Washington.

WHC. 1995. Tank Waste Remediation System Risk Management List. WHC-SD-WM-RPT-201, Westinghouse Hanford Company, Richland, Washington.

WHC. 1996. A Safety Assessment for Saltwell Jet Pumping Operations in Tank 241-A-101: Hanford Site, Richland, Washington. WHC-SD-WM-SAD-034 Rev 0, Westinghouse Hanford Company, Richland, Washington.

Wheeler TA, SC Hora, WR Cramond, and SD Unwin. 1989. Analysis of Core Damage Frequency from Internal Events: Expert Judgment Elicitation. NUREG-CR 5116, Vol. 1 and 2, Sandia National Laboratories, Albuquerque, New Mexico.

Wood TW, VL Hunter, and JW Ulvila. 1995. A Value of Information Approach to Data Quality Objectives for the Hanford High-Level Waste Tanks. PNL-SA-25649, Pacific Northwest Laboratory, Richland, Washington. 
Appendix A

Risk Constraint Measures 



\section{Appendix A}

\section{A.1 Risk Constraint Measures for Routine Operations and Post-Remedial Conditions}

Definition: The Risk Constraint level is a level of risk (or dose, exposure, or other impacts) above which some regulatory response, technical or scientific objection, or public protest is typically triggered.

Examples include exceedance of a regulatory standard, an accidental release that requires some minimal level of emergency response, a significant likelihood of project delay and inquiry, or residual contaminant levels that invoke some level of restricted access or land use. The middle column of Table A.1 may be thought of as things to minimize, avoid, or (in a few instances) goals to achieve. Before the Constraint Measures are used, they must be reviewed for verbal precision; for example, are the constraint levels definite levels that must not be reached, or do they define the minimum level of impact that is likely to be acceptable? Note that for more significant events, additional measures may need to be evaluated, because there could be many more adverse impacts whose effects persist longer. The constraint levels listed in Table $A .1$ are minimum-impact definitions. 
Table A.1. Risk Constraint Measures for Routine Operations and Post-Remedial Conditions

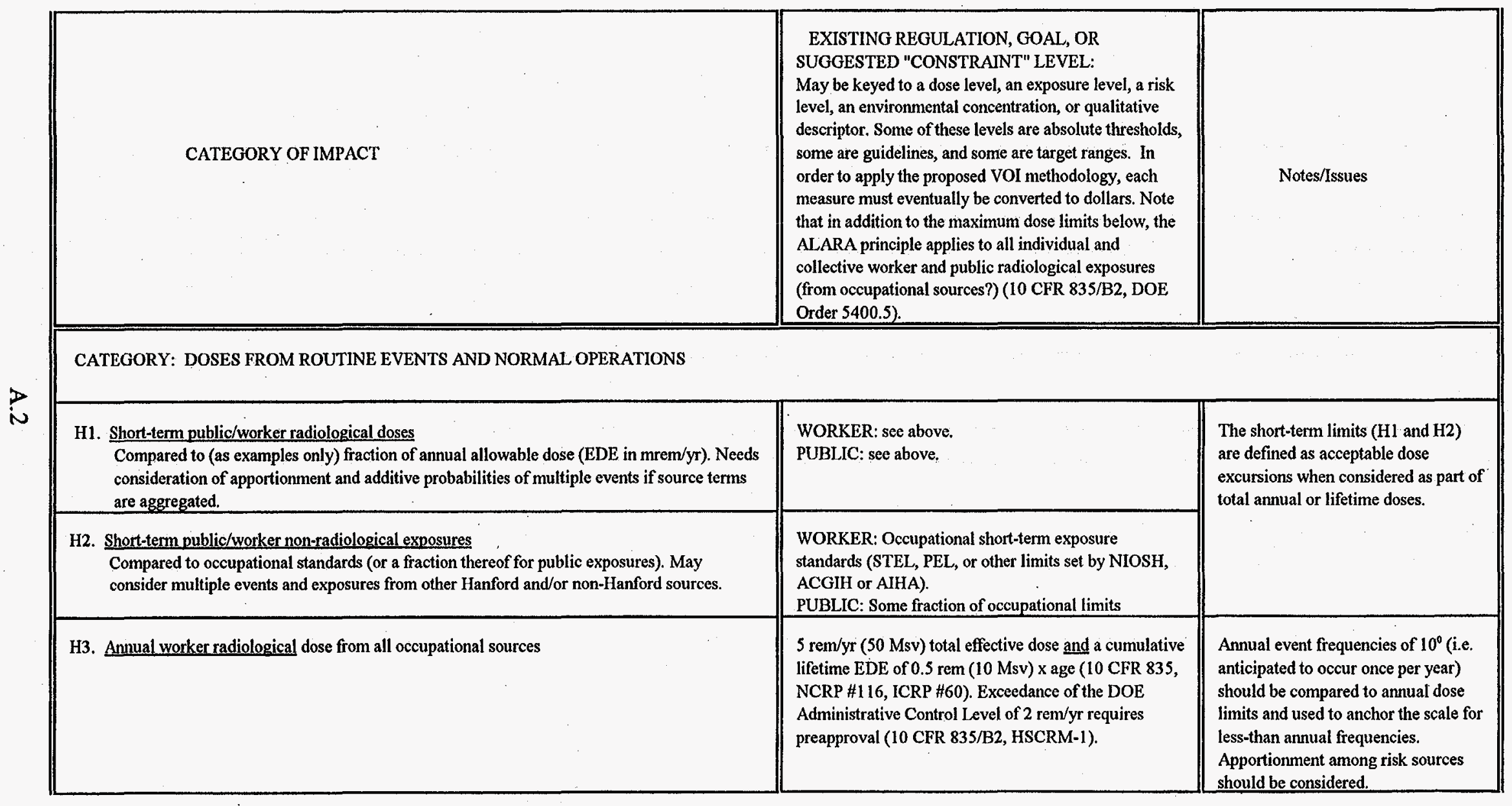




\section{H4. Annual public (individual) radiological dose}

Contribution ("apportionment") to the annual public MEI radiological Hanford-wide tota dose and inhalation dose from routine (stack + fugitive) and unplanned releases. Includes consideration of tanks as point sources, privatized processing activities as contributing to Hanford air quality, etc.
Public MEI dose limit (TEDE) $=100 \mathrm{mrem} / \mathrm{yr}(1$ $\mathrm{Msv} / \mathrm{yr}$ ), summed across all continuous or frequent

exposures from all sources (for local compliance this is all Hanford sources combined), all pathways, and all

radionuclides; $10 \mathrm{mrem} / \mathrm{yr}$ of this dose may be by

inhalation (40 CFR 60 - NESHAPs; NCRP \#116; 10

CFR 23; 40 CFR 191.04; DOE Order 5400.5; Draft

10 CFR 834). There are additional limits, such as 0.5

Msv/month ( $50 \mathrm{mrem} / \mathrm{mo})$ for the embryo-fetus.

Also: $25 \mathrm{mrem} / \mathrm{yr}(0.25 \mathrm{Msv} / \mathrm{yr}$ ) (or $15 \mathrm{mrem} / \mathrm{yr}$ as

cited by EPA in 40 CFR 191 for WIPP) whole body

dose from a single source, termed "apportionment" (40

CFR 191, 10 CFR 61.41, 40 CFR 190; 40 CFR

191.03; WAC 173-480, NCRP \#116).

Draft 40 CFR 193 \& 196 have limits of 15 and 75

mrem/yr, depending on "protection" (??).

For HLW storage: $15 \mathrm{mrem} / \mathrm{yr}$ for 10,000 yrs ( $40 \mathrm{CFR}$

191.15)

Groundwater ingestion dose for an individual located

offsite during facility operations and at $100 \mathrm{~m}$ from the

edge of the facility after the period of institutional

control $=4 \mathrm{mrem} / \mathrm{yr}$ (DOE 5480.2A, 40 CFR 193 draft, Clean Water Act)

H5. Annual population radiological dose

\section{TBD}

Collective $\mathrm{EDE}=500$ person-rem/yr $(\mathrm{DOE} 5820.2 \mathrm{~A}$ groundwater only?)
Same comment as above.

Issue: current Hanford MEI location may eventually move on-site to 100

$m$ or at the 200 Area boundary.

There are nuances in public dose limits for HLW/LLW storage, operational facilities, and so on

Issues: this measure may need to be applied to a subset of the general

population which has high exposure

through reasonably anticipated land

uses; is this the $80 \mathrm{~km}$ population? 


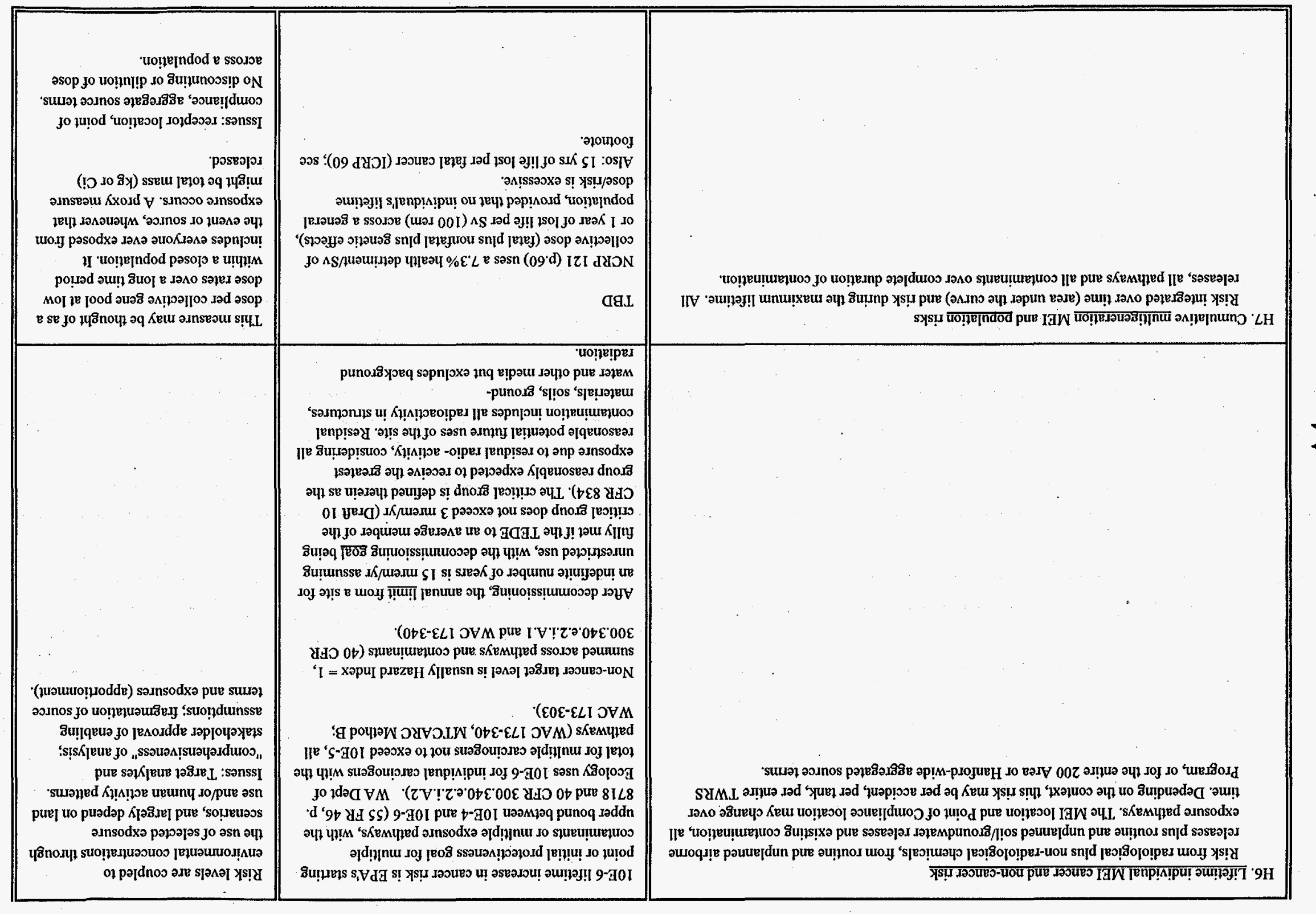


CATEGORY: ENVIRONMENTAL IMPACTS (due to any type of release and response to it)

E1. Contamination of media

Soil, groundwater, air, surface water concentrations relative to regulatory standard (various statutes) or antidegradation policies (e.g. GWPMS). May include provisions for multiple contaminants, aggregate area source terms (permitted plus unplanned releases), and multiple events/releases.

\section{E2. Ecotoxicity and habitat impacts}

Contamination of living natural resources (ecotoxicity) and/or impacts to ecosystem and habitat functions and services. Includes measures for reversibility, organism-populationcommunity scaling, habitat and landscape functions, aesthetics, the potential for contaminant migration, foodchain biomagnification, population vitality, the impact on species' gene pools, additive effects with other stressors and other actions/releases, and interfaces between habitat types and trophic levels. Also includes consideration of meeting Trusteeship responsibilities. (General citations are given in this column because specific measures and reporting requirements are not specified: NEPA, NRDA - 42 USC 9601 Section 107.a.4.c, ESA - 16 USC 1531, 16 USC 661, 16 USC 668, 16 USC 703, 16 USC 1271, 16 USC 757a, 43 USC 1701.a.8)

E3. Environmental impacts from remediation or emergency response actions Additional physical impacts from remediation or responding to an accident (including restoration costs, loss of future use and impaired aesthetics due to habitat loss, and permanent residual loss or reduction of resource services after restoration). Includes provision for adding impacts of multiple individual events, as well as for increasing remediation costs if release is not prevented, increasing physical remediation harm due to contamination spreading or plume mixing, and additional acreage needed for disposal needed above technical planning basis. (citations as above, plus cleanup regulations).
Avoid any release or exceedance of a regulatory standard which triggers program or regulatory response, Notice of Violation of an operating permit, land impoundment or food interdiction (by the WA Dept of Health), or new restriction on access due to exceedance of a regulatory standard.

Only localized $\left(<1000 \mathrm{~m}^{2}\right)$ short-term effects $(<1$ yr or season) (worse: widespread short-term or widespread, long-term and/or irreversible).

Only impacts on individual organisms of non-T\&E species, but not on populations, communities or ecosystems. Dose to individual non-T\&E organisms less than $1 \mathrm{rad} / \mathrm{d}$.

T\&E species: no "taking" is allowed, including incidental taking via harassment or habitat disturbance.

No or low potential for contaminant migration

Removal or disturbance of surface soil $<1000 \mathrm{~m}^{2}$ required over the lifetime of the facility or the ful remedial cycle.
Issues: Single or multiple contaminants; aggregation of source terms; dilution as the solution; definition of "degradation;" detection limits; definition of a "significant" duration of impact. Both the degree

of exceedance and the duration of contamination are important to know.

For a minimum level of impact, it is assumed that impacts to individual organisms would not result in substantive impacts to ecosystem functions and services. For more significant impacts, other measures of ecosystem functions and services need to be included.

Issues: definition of baseline or optimal conditions; intermeshed spatial and temporal scales: overlapping transport and transfer pathways; selection of indicator or

surrogate species; representativeness of simplified measures or biomarkers.

Issues: effectiveness of restoration; how satisfactory is mitigation; condition of media (such as soil column profile) and biota after completion of action relative to target or optimal conditions; uniqueness and functional criticality of the area/habitat type. 


\begin{tabular}{|c|c|c|}
\hline $\begin{array}{l}\text { S1. Community quality of life } \\
\text { Impacts on quality of life and the economic base, or the demographic characteristics of the } \\
\text { local community (NEPA - } 42 \text { USC } 4321 \text { et seq; specific measures not specified). Includes } \\
\text { benefits of an intact/uncontaminated environment, aesthetics, and recreational quality. (see } \\
\text { footnote for additional measures). }\end{array}$ & $\begin{array}{l}<5-10 \% \text { adverse impacts on jobs, markets, the } \\
\text { community or publicity. } \\
\text { Perceptible loss of recreational opportunities and } \\
\text { aesthetics or quality of experience. }\end{array}$ & $\begin{array}{l}\text { These impacts must be included even } \\
\text { if they occur in response to } \\
\text { "perceived" risk to local quality of } \\
\text { life. Community values are real } \\
\text { things at real risk even from } \\
\text { seemingly small occurrences. } \\
\text { Issues: reversibility (i.e. duration of } \\
\text { restricted access or environmental or } \\
\text { aesthetic decrement); distribution of } \\
\text { impacts versus benefits. }\end{array}$ \\
\hline $\begin{array}{l}\text { S2. Tribal quality of life } \\
\text { Impacts to tribal culture, health, quality of and access io natural and cutturat resources, } \\
\text { quality of life and socio-economics. Includes benefits of an intact/uncontaminated } \\
\text { environment, aesthetics and other measures (NEPA, Treaties with each American Indian } \\
\text { Nation, Executive Order 12898, DOE American Indian Policy, DOE Order 1230.2, Acts } \\
\text { listed under S4). (footnote) }\end{array}$ & $\begin{array}{l}\text { No/minimal restriction on access, but increased dose } \\
\text { over background if used. } \\
\text { No key culturally-important resource or site whose } \\
\text { character requires non-disturbance, non-defilement, or } \\
\text { non-degradation is contaminated or physically harmed. }\end{array}$ & $\begin{array}{l}\text { Other measures for assessing impacts } \\
\text { to socio-cultural quality of life are } \\
\text { also available. } \\
\text { Issues: duration or permanence of } \\
\text { harm; definition of cultural site (e.g. } \\
\text { gravesite), traditional cultural } \\
\text { property, culturally-important } \\
\text { resources, and historically-important } \\
\text { landscapes (see Park Service } \\
\text { Bulletins); definition of harm (i.e. } \\
\text { contamination versus physical } \\
\text { disturbance). }\end{array}$ \\
\hline $\begin{array}{l}\text { S3. Intra- and inter-generational equity } \\
\text { (What members of the present generation are at greatest risk of exposure and risk of effects, } \\
\text { and why? Is the degree of health protectiveness evenly distributed across all reasonably } \\
\text { expected exposed groups? Whose resources and activities are most likely to be harmed by } \\
\text { releases and remediation? Does a decision alternative result in essentially irretrievable waste } \\
\text { forms, or does it decrease future land use options? Will the cleanup/disposal burden increase } \\
\text { if not dealt with soon? Do management goals promote sustainability of the habitat, resources } \\
\text { and uses?) (NEPA, Executive Order 12898, others) }\end{array}$ & $\begin{array}{l}<10 \% \text { of the most-impacted significant Site user group } \\
\text { is adversely affected. Contamination and harm is } \\
\text { limited to the present generation. Minimally increased } \\
\text { future long-term cleanup and monitoring costs. } \\
\text { Minimally increased storage and disposal capacity and } \\
\text { total quantity of waste left on Site; can be } \\
\text { accommodated under current plans. (NEPA, Executive } \\
\text { Order 12898) }\end{array}$ & $\begin{array}{l}\text { Issues: intrusion safety at } 100 \text { and } \\
500 \text { years; whether to allow } \\
\text { discounting. }\end{array}$ \\
\hline $\begin{array}{l}\text { S4. Cultural resource impacts from remediation or emergency response actions } \\
\text { Additional physical disturbance of cultural, historic or archaeological resources during } \\
\text { remediation or emergency response (NHPA - 16 USC 470, ARPA - 16 USC 470aa-470.11, } \\
\text { AIRFA - } 42 \text { SC 1996, NAGPRA - PL 101-601, 16 USC 431-433, 36 CFR Parts 60, 63, 79, } \\
\text { 800,). }\end{array}$ & $\begin{array}{l}\text { No or minimal perturbation or disturbance of site(s) or } \\
\text { resource(s) during response to the event. } \\
\text { (NHPA - } 16 \text { USC } 470 \text {, ARPA - } 16 \text { USC } 470 \text { aa- } \\
470.11 \text {, ARRA - } 42 \text { SC } 1996 \text {, NAGPRA - PL 101- } \\
601,16 \text { USC } 431-433,36 \text { CFR Parts } 69,63,79 \text { abd } \\
800 \text { ). }\end{array}$ & Issues: mitigation versus protection. \\
\hline
\end{tabular}


CATEGORY: PROGRAMMATIC IMPACTS (of inadequate characterization, of accident, of environmental contamination)

P1. Non-compliance penalties

Potential for, and degree of violations, penalties, or threat of litigation due to non-compliance with statutes, orders, permits, treaties, Tri-Party Agreement, Trusteeship, or other.

P2. Shutdown costs

Shutdown/Standdown costs if safety issue not resolved, if accident occurs, if retrieval is impeded through inadequate characterization, etc.

P3. Response and repair costs

Response and repair costs and scheduling impacts to reestablish or prolong MinSafe conditions during interim storage or retrieval/disposal.

P4. Mission goals and milestones

Impacts on the timely ability of the program or facility to meet its missions of safe interim storage, volume-based retrieval, waste processing/disposal, and clean closure.

P5. Inter-program and Sitewide ramifications

Scheduling impacts and ramifications for other activities or programs, including privatization and disposal, and effects on the ability of other programs/activities to meet their TPA milestones.

P6. Stakeholder acceptance and implications to the program(s)

Impact on implementation and credibility if stakeholders are not involved early and honestly in the decision process; risk of delay if stakeholders disagree with performance measures or

retrieval strategy (this is not the same as "risk communication" or informational briefings,

but assesses the true degree of partnership and co-decision making).
Only marginal noncompliance with orders etc or

marginal administrative noncompliance without

potential for fines, jail, permit denial or compensatory requirements.

$<\$ 0.5$ Million (RDS guideline)

$<\$ 0.5$ Million (RDS Guideline)

Goals and milestones can be met with minor

adjustments within the activity/facility.

Goals and milestones can be met with minor

adjustments within the program or between or among

programs.

Stakeholders are an integral part of the decision process

so assumptions are likely to be acceptable and the

decision is likely to be durable.

\begin{tabular}{||l||}
\hline \\
\hline \\
\hline \\
\hline \\
\hline
\end{tabular}


For workers, a single uniform whole body dose of $1 \mathrm{~Sv}(100 \mathrm{mrem})=4 \mathrm{E}-2$ lifetime fatal cancer risk $+0.8 \mathrm{E}-2$ severe genetic defects $+0.8 \mathrm{E}-2$ non-fatal cancer risk $=5.6 \mathrm{E}-2$ total lifetime detriment . (NCRP \#1 16, p.30, 1993). For the public, $1 \mathrm{~Sv}(100 \mathrm{rem})=5 \mathrm{E}-2$ fatal cancer risk, $1.3 \mathrm{E}-2$ for serious genetic effects, and $1 \mathrm{E}-2$ for non-fatal cancer, resulting in a total detriment of $7.3 \mathrm{E}-2 / \mathrm{Sv}$ (NCRP $\# 116$, p. 51). The difference between the worker and public dose-to-cancer risk conversions are due to the age distribution in the worker versus the general population and also to the duration of the committed doses ( $50 \mathrm{yr}$ for workers and $70 \mathrm{yrs}$ for the public).

$1 \mathrm{rem}=0.01 \mathrm{Msv} ; 1 \mathrm{mSv}=100 \mathrm{mrem} ; 1 \mathrm{~Sv}=100 \mathrm{rem}$

De minimis cancer risk levels are typically assumed to be $10 \mathrm{E}-6$ or 10E-7 excess lifetime cancer risk due to the single source. The "negligible individual risk level" is defined as 1 to $5 \mathrm{x} 10 \mathrm{E}-7 / \mathrm{yr}$ (equivalent to $0.01 \mathrm{mSv} / \mathrm{yr}$ or $1 \mathrm{mrem} / \mathrm{yr}$ ) per source or practice (NCRP \#116, p.52).

Note $\mathrm{H} 1$ and $\mathrm{H} 2$ : When considering risk from acute exposures (or short-term visits), there may be a potential problem if an annual dose limit or a fraction thereof is used in a manner which restricts the number of visits allowed by a member of the public per year. In addition, if activities such as certain cultural practices require visits to a location that is within a partially restricted zone, the issue of whether there is a risk burden due to the probability of an event even no event or no exposure occurs. At some level of high probability, the risk burden may become unacceptable.

DOE-SEN-35-91 contains general risk goals relative to individual and population rates of other accidental and cancer deaths:

- The risk to an average individual in the vicinity of a DOE nuclear facility for prompt fatalities that might result from accidents should not exceed $0.1 \%$ of the sum of prompt fatalities resulting from other accidents to which members of the general population are generally exposed. For evaluation purposes, individuals are assumed to be located within one mile of the site boundary.

- The risk to the population in the area of a DOE nuclear facility for cancer fatalities that might result from operations should not exceed $0.1 \%$ of the sum of all cancer fatality risk resulting from other causes. For evaluation purposes, individuals are assumed to be located within ten miles of the site boundary.

Note $\mathbf{S 1}$ and $\mathbf{S 2 :}$ Additional Quality of Life measures:

- Economic well-being: community costs for services, effects on housing markets, income or tax or bankruptcy rates, net job differential, health care costs, lost productivity, wellfunctioning infrastructure, access to adequate education, costs of avoiding exposure or illness (neg.).

- Community well-being: good mental health, trust of governing institutions, access to reliable information, personal security, low stress levels, assurance for the well-being of future generations, funding stability, sustainable economic and environmental practices, capturing economic opportunity in the local community, shared decision-making power, equitable distribution of impacts and benefits, community cohesiveness, accountability of decision makers, connection to the land with local access to open space, protection of cultural and religious values, degree of ceremonial quality of the site/resource relative to optimal and adequacy of aesthetic buffer zones, quality of religious (or recreational) experience at an alternative site, cost and inconvenience of an alternative site or resource of equal quality, degree of spiritual integrity of the overall traditional/heritage area, individual and collective psycho-social well-being derived from membership in a healthy community with access to ancestral lands and heritage resources, degree (and effectiveness) of protection and preservation efforts being expended to maintain good conditions or restore lost quality and use, ability to satisfy the personal responsibility for maintenance of the spiritual quality of a site/resource/area and the responsibility to participate in traditional practices as a community member, quality of the socio-cultural and eco-cultural landscapes, intergenerational transfer of community educational and cultural knowledge 


\section{A.2 Risk Constraint Measures for Accident Conditions by Severity Class}

This section assumes that there is an inverse correlation between event frequency and severity class (see Table A.2). Descriptors for each measure are expressed in terms of severity of impact in Table A.3. Some of the severity descriptions are numerical, while some are still in narrative form until numerical correlations can be developed jointly with reguators and stakeholders.

Table A.2. Event Frequency and Severity Class

\begin{tabular}{||l|l|}
\hline $\begin{array}{c}\text { EVENT FREQUENCY } \\
\text { (with Event Frequency Category } \\
\text { from WHC-CM-4-46) }\end{array}$ & $\begin{array}{c}\text { SEVERITY CLASS } \\
\text { (with Hazard Category from WHC-CM- } \\
4-46, \text { Rev-4) }\end{array}$ \\
\hline $\begin{array}{l}\text { Normal operations (10\%/year } \\
\text { frequency of routine excursions) }\end{array}$ & $\begin{array}{l}\text { Acceptable, or within the Safe or Clean } \\
\text { compliance envelope } \\
\text { (this report's definition) }\end{array}$ \\
\hline $\begin{array}{l}10^{-2} \text { to }<10^{\circ} / \text { year } \\
\text { ("Anticipated") }\end{array}$ & $\begin{array}{l}\text { Low } \\
\text { (Category 3 Hazard: only significant } \\
\text { localized consequences) }\end{array}$ \\
\hline $\begin{array}{l}10^{-4} \text { to } \leq 10^{-2} / \text { year } \\
\text { "Unlikely") }\end{array}$ & $\begin{array}{l}\text { Moderate } \\
\text { (Category 2 Hazard: significant onsite } \\
\text { consequences) }\end{array}$ \\
\hline $\begin{array}{l}10^{-6} \text { to } \leq 10^{-4} / \text { year } \\
\text { ("Extremely Unlikely") }\end{array}$ & $\begin{array}{l}\text { High/Catastrophic } \\
\text { (Category 1 Hazard: significant offsite } \\
\text { consequences) }\end{array}$ \\
\hline $\begin{array}{l}\text { Note: The hazard category is used to apply a graded level of safety analysis and } \\
\text { may not reflect the actual severity of unmitigated consequences (WHC-CM-4-46, } \\
\text { Section 3.2) }\end{array}$ \\
\hline
\end{tabular}


Table A.3. Risk Constraint Measures for Accident Conditions by Severity Class

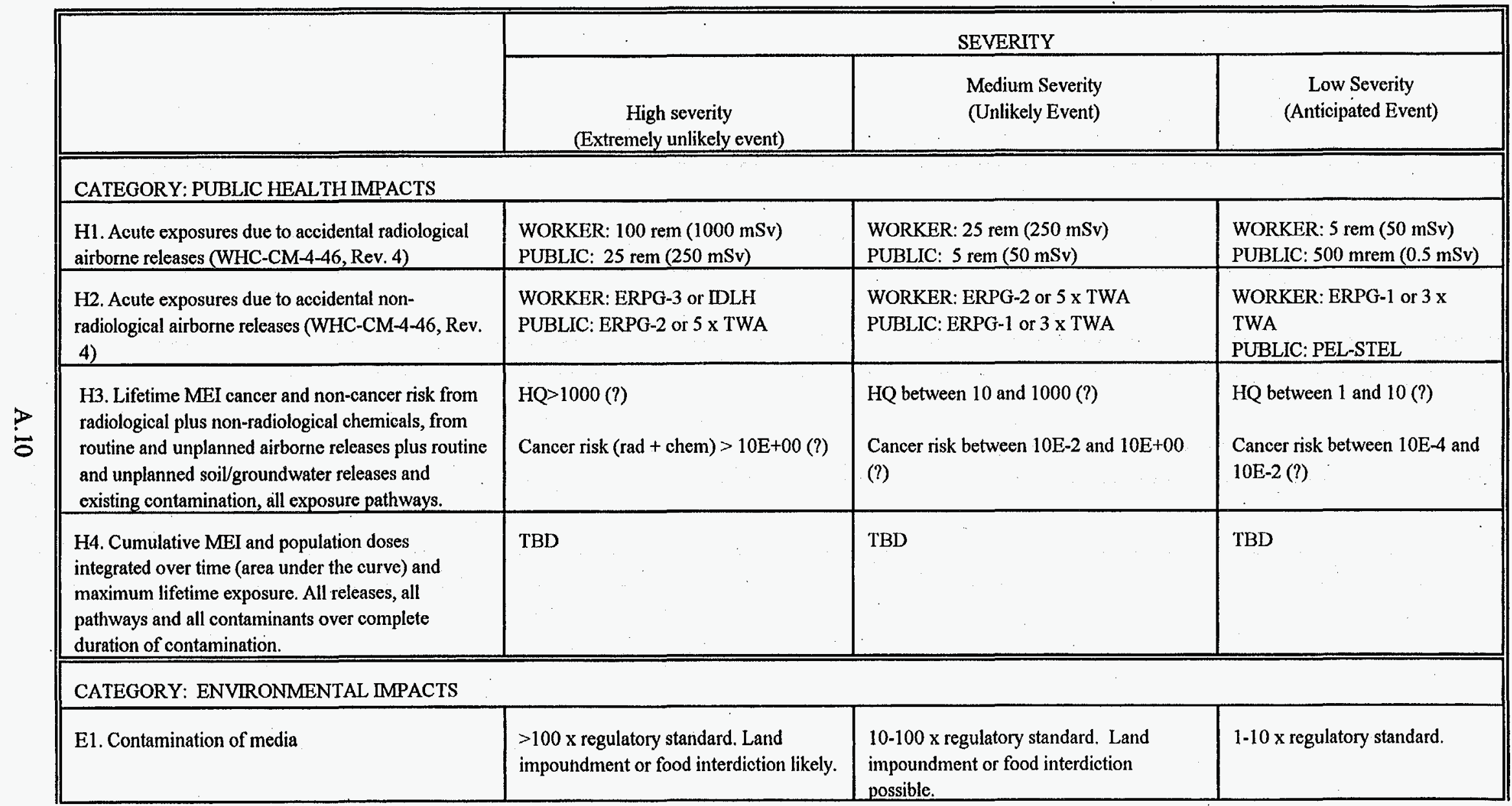




\begin{tabular}{|c|c|c|c|}
\hline $\begin{array}{l}\text { E3. Additional physical impacts from responding to } \\
\text { the action (including restoration costs and permanent } \\
\text { loss or reduction of resource services after } \\
\text { restoration). }\end{array}$ & $\begin{array}{l}\text { Removal or disturbance of surface soil } \\
>10,000 \mathrm{~m}^{2} \text { required. }\end{array}$ & $\begin{array}{l}\text { Removal or disturbance of surface soil } \\
1,000 \text { to } 10,000 \mathrm{~m}^{2} \text { required. }\end{array}$ & $\begin{array}{l}\text { Removal or disturbance of } \\
\text { surface soil }<1,000 \mathrm{~m}^{2} \text { required. }\end{array}$ \\
\hline \multicolumn{4}{|l|}{ CATEGORY: SOCIO-CULTURAL IMPACTS } \\
\hline S2. Tribal quality of life & $\begin{array}{l}\text { Loss of access }>10 \text { yrs. (acreage TBD) } \\
\text { Culturally-important resource/site } \\
\text { contaminated or physically harmed } \\
\text { irreversibly or irretrievably. }\end{array}$ & $\begin{array}{l}\text { Lost access } 1-10 \text { yrs. } \\
\text { Culturally-important resource/site } \\
\text { contaminated or physically harmed } \\
\text { significantly but not permanently. }\end{array}$ & $\begin{array}{l}\text { No lost access, but increased } \\
\text { dose if used. } \\
\text { Culturally-important } \\
\text { resource/site contaminated or } \\
\text { physically harmed to any degree. }\end{array}$ \\
\hline
\end{tabular}




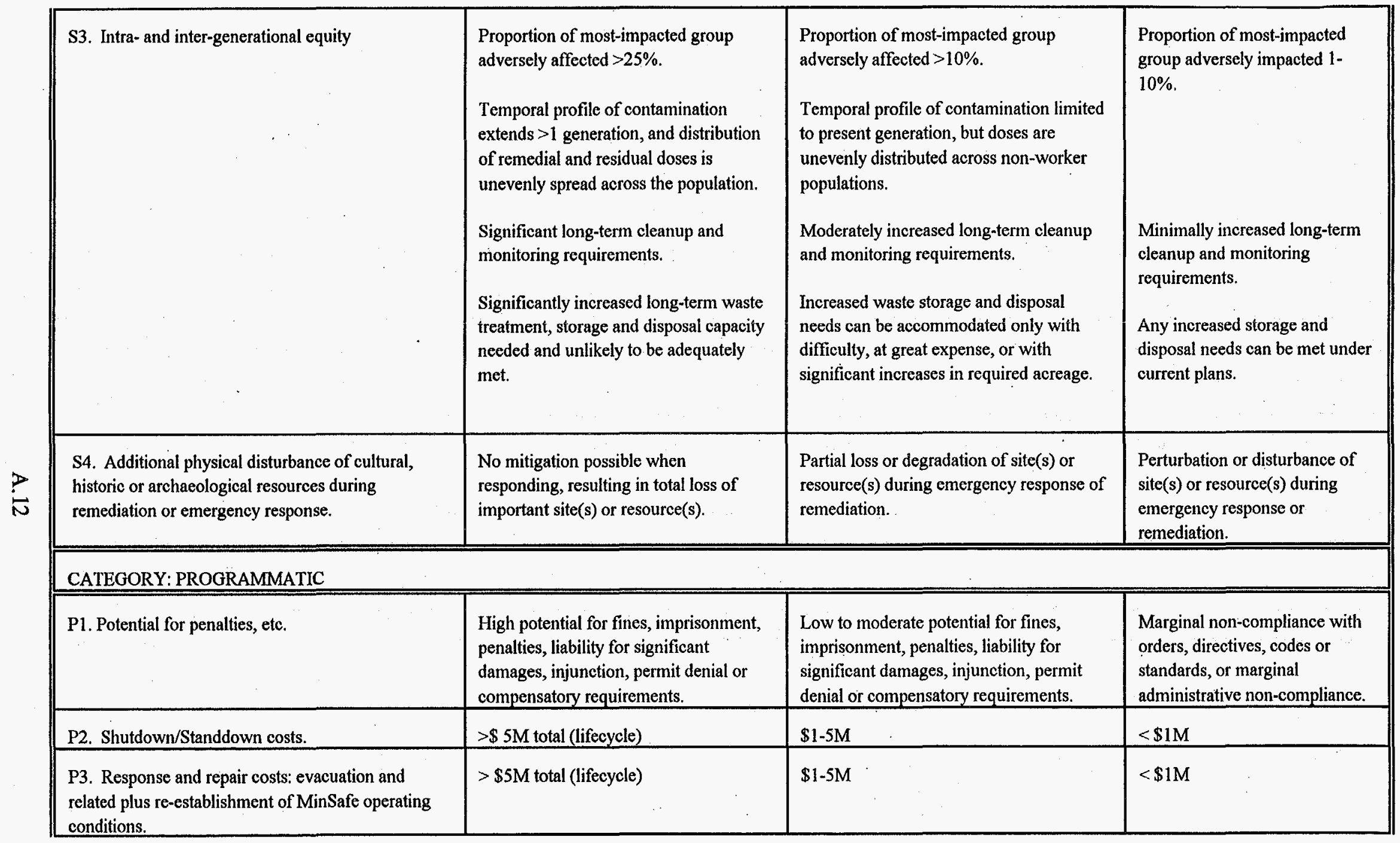




\begin{tabular}{|c|c|c|c|}
\hline $\begin{array}{l}\text { P4. Impacts on the ability of the program to meet its } \\
\text { mission. }\end{array}$ & $\begin{array}{l}\text { Serious impact to overall performance } \\
\text { evaluation or the ability to meet annual } \\
\text { technical performance goals and } \\
\text { milestones } \\
\text { Program reorganization likely, with } \\
\text { federal and state investigation and } \\
\text { certainty audits; major rebaselining } \\
\text { probably reburied. } \\
\text { Significant violation of public trust and } \\
\text { confidence. } \\
\text { Threats to funding stability. }\end{array}$ & $\begin{array}{l}\text { Moderate interruption where goals and } \\
\text { milestones may still be met. } \\
\text { Reorganization and audits possible; } \\
\text { program evaluation efforts and increased } \\
\text { scrutiny will occur. } \\
\text { Significant erosion of image and } \\
\text { credibility. } \\
\text { Funding maintained but still at risk. }\end{array}$ & $\begin{array}{l}\text { Goals and milestones can be met } \\
\text { with minor adjustments. }\end{array}$ \\
\hline $\begin{array}{l}\text { P5. Schedule impacts and ramifications for other } \\
\text { activities or programs, including privatization and } \\
\text { disposal. }\end{array}$ & $\begin{array}{l}>10 \% \text { chance of }>1 \mathrm{yr} \text { increase in } \\
\text { schedule or } 1 / 1000 \text { chance of }>10 \mathrm{yr} \\
\text { increase in schedule. }\end{array}$ & $\begin{array}{l}\text { Milestone slips with some impact on other } \\
\text { programs and activities, but overall } \\
\text { Hanford schedule is maintained. }\end{array}$ & $\begin{array}{l}\text { Milestone schedule is } \\
\text { maintained by intra- and inter- } \\
\text { program adjustments. }\end{array}$ \\
\hline \multicolumn{4}{|c|}{$\begin{array}{l}\text { Notes: } \\
\text { Human Health: ERPG-1 = the maximum airborne concentration below which it is believed that nearly all individuals could be exposed for up to } 1 \mathrm{hr} \text { without experiencing other than } \\
\text { mild transient adverse health effects or perceiving clearly defined objectionable odor. ERPG- } 2=\text { the maximum airborne concentration below which it is believed that nearly all } \\
\text { individuals could be exposed for up to } 1 \mathrm{hr} \text { without experiencing or developing irreversible or other serious health effects or symptoms that could impair their abilities to take } \\
\text { protective action. ERPG-3 = the maximum airborne concentration below which it is believed that nearly all individuals could be exposed for up to } 1 \mathrm{hr} \text { without experiencing or } \\
\text { developing life-threatening health effects. PEL-STEL = permissible exposure level, short term ( } 15 \text { min) exposure level for occupational settings. PEL-TWA = permissible exposure } \\
\text { level, time weighted average for occupational settings. IDLH = immediately dangerous to life and health. } \\
\text { Environmental Impacts: Effects on individual organisms might include a fraction of a lethal dose to one or more sensitive species or to threatened or endangered species. } \\
\text { Effects on population vitality might include organism density relative to optimal density for that area. Community effects might include the structure and function of the } \\
\text { community as well as the ability of the community to provide physical, eco-cultural or aesthetic functions and services. } 10,000 \mathrm{~m}^{2} \text { is the homerange area of a loggerhead } \\
\text { shrike. }\end{array}$} \\
\hline
\end{tabular}


Appendix B

Condensed Phase Organic Reactions Safety Issue Information 


\section{Appendix B}

Table B.1. Condensed Phase Organic Nitrate Safety Issue Prior Probability of Distributions of Tank Contents for Saltcake Tanks

\begin{tabular}{|c|c|c|c|c|c|c|c|c|c|c|c|c|c|c|c|}
\hline \multirow[t]{2}{*}{ Tank } & \multicolumn{2}{|c|}{$\begin{array}{c}\text { Threshold Volumes } \\
\left(\mathrm{m}^{3}\right)\end{array}$} & \multicolumn{13}{|c|}{ Prior Probability of Tank Contents in each Region } \\
\hline & HEPA & Dome & A & B & $\mathrm{C}$ & $\mathrm{D}$ & $\mathrm{E}$ & $\mathbf{F}$ & G & $\mathrm{H}$ & I & $\mathrm{J}$ & K & $\mathbf{L}$ & $\mathrm{M}$ \\
\hline A101 & 0.1338 & 0.6692 & 0.747 & 0.000 & 0.000 & 0.136 & 0.000 & 0.000 & 0.113 & 0.000 & 0.000 & 0.004 & 0.000 & 0.000 & 0.000 \\
\hline 1102 & 4791 & 2.3954 & 0.984 & 0.000 & 0.000 & 0.015 & 0.000 & 0.000 & 0.001 & 0.000 & 0.000 & 0.000 & 0.000 & 0.000 & \\
\hline $\mathrm{AX} 101$ & 0.2114 & 0572 & 0.797 & 0.000 & 0.000 & 0.096 & 0.000 & 0.000 & 0.097 & 0.000 & 0.000 & 0.005 & 0.000 & 0.000 & 0.005 \\
\hline $\mathrm{AX102}$ & 0.4798 & 2.3992 & 0.981 & 0.000 & 0.000 & 0.015 & 0.000 & 0.000 & 0.004 & 0.000 & 0.000 & 0.000 & 0.000 & 0.000 & 10 \\
\hline$\Delta \times 103$ & 4522 & 261 & 0.933 & 0.000 & 0.000 & 0.050 & 0.000 & 0.000 & 0.017 & 0.000 & 0.000 & 0.000 & 0.000 & 0.000 & 0.0 \\
\hline 3102 & 0.2929 & 1.4644 & 0.991 & 0.000 & 0.000 & 0.008 & 0.000 & 0.000 & 0.001 & 0.000 & 0.000 & 0.000 & 0.000 & 0.000 & 0.0 \\
\hline 104 & 646 & 0.8228 & 0.914 & 0.000 & 0.000 & 0.052 & 0.000 & 0.000 & 0.034 & 0.000 & 0.000 & 0.000 & 0.000 & 0.000 & 0.0 \\
\hline 3105 & 0.1892 & 0.9458 & 0.877 & 0.000 & 0.000 & 0.079 & 0.000 & 0.000 & 0.041 & 0.000 & 0.000 & 0.002 & 0.000 & 0.000 & 0.0 \\
\hline BX105 & 88 & 1.4285 & 0.999 & 0.000 & 0.000 & 0.001 & 0.000 & 0.000 & 0.000 & 0.000 & 0.000 & 0.000 & 0.000 & 0.000 & 0.00 \\
\hline$B \times 110$ & 0.2266 & 1.1332 & 0.999 & 0.000 & 0.000 & 0.001 & 0.000 & 0.000 & 0.000 & 0.000 & 0.000 & 0.000 & 0.000 & 0.000 & 0.8 \\
\hline$B \times 111$ & 0.2437 & 1.2184 & 0.902 & 0.000 & 0.000 & 0.066 & 0.000 & 0.000 & 0.032 & 0.000 & 0.000 & 0.000 & 0.000 & 0.000 & 0.00 \\
\hline BY101 & 0.2535 & 1.2675 & 0.055 & 0.000 & 0.000 & 0.071 & 0.000 & 0.000 & 0.424 & 0.000 & 0.000 & 0.215 & 0.000 & 0.000 & 0.2 \\
\hline BY102 & 0.2709 & 1.3546 & 0.855 & 0.000 & 0.000 & 0.083 & 0.000 & 0.000 & 0.060 & 0.000 & 0.000 & 0.002 & 0.000 & 0.000 & 0.000 \\
\hline BY103 & 0.2486 & 1.2429 & 0.849 & 0.000 & 0.000 & 075 & 0.000 & 0.000 & 0.073 & 0.000 & 0.000 & 0.003 & 0.000 & 0.000 & 0.0 \\
\hline BY104 & 0.2463 & 1.2316 & 0.011 & 0.000 & 0.000 & 0.022 & 0.000 & 0.000 & 0.304 & 0.000 & 0.001 & 0.243 & 0.000 & 0.000 & 0.419 \\
\hline BY105 & 2096 & 1.048 & 0.835 & 0.000 & 0.000 & 088 & 0.000 & 0.000 & 0.069 & 0.000 & 0.000 & 0.004 & 0.000 & 0.000 & \\
\hline BY106 & 0.157 & 0.7849 & 0.795 & 0.000 & 0.000 & 0.090 & 0.000 & 0.000 & 0.107 & 0.000 & 0.000 & 0.008 & 0.000 & 0.000 & 0.000 \\
\hline BY107 & 3 & 1.4965 & 0.075 & 0.000 & 0.000 & 090 & 0.000 & 0.000 & 0.457 & 0.000 & 0.002 & 0.229 & 0.000 & 0.002 & 0.1 \\
\hline BY108 & 0.3137 & 1.5685 & 0.124 & 0.000 & 0.000 & 0.166 & 0.000 & 0.000 & 0.582 & 0.000 & 0.001 & 0.119 & 0.000 & 0.000 & 0.0 \\
\hline BY109 & 0.239 & 1.1994 & 0.862 & 0.000 & 0.000 & 073 & 0.000 & 0.000 & 0.061 & 0.000 & 0.000 & 0.004 & 0.000 & 0.000 & 0.0 \\
\hline BY110 & 0.2493 & 1.2467 & 0.053 & 0.000 & 0.000 & .067 & 0.000 & 0.000 & 0.387 & 0.000 & 0.001 & 0.235 & 0.000 & 0.001 & 0.2 \\
\hline BY111 & 0.22 & 1.1312 & 0.036 & 0.000 & 0.000 & .053 & 0.000 & 0.000 & 0.314 & 0.000 & 0.001 & 0.230 & 0.000 & 0.004 & 0.3 \\
\hline BY112 & 0.2898 & 1.4492 & 0.056 & 0.000 & 0.000 & 0.084 & 0.001 & 0.002 & 0.384 & 0.000 & 0.000 & 0.244 & 0.000 & 0.002 & 0.2 \\
\hline$S 101$ & 0.2384 & 1.1918 & 0.247 & 0.000 & 0.000 & 0.181 & 0.000 & 0.000 & 0.467 & 0.000 & 0.000 & 0.085 & 0.000 & 0.001 & 0.0 \\
\hline $\mathrm{S} 102$ & 0.1922 & 0.9609 & 0.143 & 0.000 & 0.000 & 0.131 & 0.000 & 0.000 & 0.457 & 0.000 & 0.000 & 0.141 & 0.000 & 0.000 & 0.1 \\
\hline$\$ 103$ & 0.3061 & 1.5306 & 0.265 & 0.000 & 0.000 & 0.188 & 0.000 & 0.000 & 0.416 & 0.000 & 0.000 & 0.107 & 0.000 & 0.000 & 0.0 \\
\hline 5105 & 0.2274 & 1.1369 & 0.030 & 0.003 & 0.007 & 0.040 & 0.036 & 0.020 & 0.308 & 0.012 & 0.050 & 0.174 & 0.020 & 0.061 & 0.239 \\
\hline$\$ 106$ & 0.21 & 1.0934 & 0.164 & 0.000 & 0.000 & 0.149 & 0.000 & 0.000 & 0.462 & 0.000 & 0.000 & 0.149 & 0.000 & 0.000 & 0.0 \\
\hline 8107 & 0.2577 & 1.2883 & 0.349 & 0.000 & 0.000 & 0.249 & 0.000 & 0.000 & 0.380 & 0.000 & 0.000 & 0.021 & 0.000 & 0.000 & 0.001 \\
\hline 5108 & 0.17 & 0.8568 & 0.140 & 0.000 & 0.000 & 0.119 & 0.000 & 0.000 & 0.441 & 0.000 & 0.000 & 0.158 & 0.000 & 0.000 & 0.142 \\
\hline $\mathrm{S} 109$ & 0.185 & 0.9249 & 0.166 & 0.000 & 0.000 & 0.121 & 0.000 & 0.000 & 0.432 & 0.000 & 0.000 & 0.148 & 0.000 & 0.000 & 0.133 \\
\hline 110 & 0.2524 & 1.2618 & 0.230 & 0.000 & 0.000 & 0.181 & 0.000 & 0.000 & 0.454 & 0.000 & 0.000 & 0.097 & 0.000 & 0.000 & 0.6 \\
\hline 111 & 0.1744 & 0.8719 & 0.022 & 0.000 & .000 & 052 & 0.000 & 0.000 & 0.455 & 0.000 & 0.000 & 0.238 & 0.000 & 0.000 & 10.233 \\
\hline
\end{tabular}

B. 1 


\begin{tabular}{|c|c|c|c|c|c|c|c|c|c|c|c|c|c|c|c|}
\hline \multirow[t]{2}{*}{ Tank } & \multicolumn{2}{|c|}{$\begin{array}{c}\text { Threshold Volumes } \\
\left(\mathrm{m}^{3}\right)\end{array}$} & \multicolumn{13}{|c|}{ Prior Probability of Tank Contents in each Region } \\
\hline & HEPA & Dome & A & B & $\mathrm{C}$ & D & $\mathrm{E}$ & F & G & $\mathrm{H}$ & I & $\mathrm{J}$ & $\mathbf{K}$ & $\mathbf{L}$ & M \\
\hline 12 & 0.202 & 0101 & 0.138 & 0.000 & 0.000 & 0.110 & 0.000 & 0.000 & 0.489 & 0.000 & 0.000 & 0.143 & 0.000 & 0.000 & 0.120 \\
\hline sX101 & 0.322 & 6099 & 0.056 & 0.000 & .001 & 0.100 & 0.000 & 0.003 & 0.435 & 0.000 & 0.008 & 0.196 & 0.000 & 0.004 & 0.197 \\
\hline $5 \times 102$ & 0.2891 & 4453 & 0.025 & 0.000 & 0.000 & 0.085 & 0.000 & 0.000 & 0.405 & 0.000 & 0.000 & 0.228 & 0.000 & 0.000 & 0.257 \\
\hline $5 \times 103$ & 0.2478 & 2389 & 0.027 & 0.000 & 0.001 & 0.060 & 0.000 & 0.002 & 0.389 & 0.000 & 0.006 & 0.211 & 0.000 & 0.003 & 0.301 \\
\hline sx104 & 0.2622 & 3109 & 0.023 & 0.000 & 0.000 & 0.053 & 0.000 & 0.001 & 0.381 & 0.000 & 0.000 & 0.231 & 0.000 & 0.001 & 0.310 \\
\hline $\mathrm{s} \times 105$ & 0.2361 & 1803 & 0.029 & 0.000 & 0.000 & 0.048 & 0.000 & 0.001 & 0.366 & 0.000 & 0.000 & 0.202 & 0.000 & 0.000 & 0.354 \\
\hline $\mathrm{s} \times 106$ & 0.2909 & 4547 & 0.038 & 0.000 & 0.001 & 0.067 & 0.001 & 0.002 & 0.419 & 0.000 & 0.003 & 0.213 & 0.000 & 0.001 & 0.255 \\
\hline TX102 & 0.3179 & 5893 & 0.061 & 0.004 & 0.012 & 0.096 & 0.037 & 0.038 & 0.414 & 0.011 & 0.049 & 0.146 & 0.006 & 0.030 & 0.096 \\
\hline TX104 & 0.3754 & 1.877 & 0.431 & 0.000 & 0.000 & 0.263 & 0.000 & 0.000 & 0.290 & 0.000 & 0.000 & 0.016 & 0.000 & 0.000 & 0.000 \\
\hline TX105 & 0.1695 & 473 & 0.017 & 0.002 & 0.003 & 0.031 & 0.030 & 0.022 & 0.273 & 0.012 & 0.048 & 0.157 & 0.032 & 0.061 & 0.312 \\
\hline$t \times 106$ & 0.2285 & 1.1426 & 0.030 & 0.003 & 0.006 & 0.039 & 0.036 & 0.025 & 0.317 & 0.020 & 0.039 & 0.179 & 0.021 & 0.050 & 0.235 \\
\hline TX107 & 8864 & 319 & 0.550 & 0.000 & 0.000 & 0.263 & 0.000 & 0.000 & 0.185 & 0.000 & 0.000 & 0.002 & 0.000 & 0.000 & 0.000 \\
\hline TX108 & 0.3493 & 1.7464 & 0.112 & 0.000 & 0.000 & 0.125 & 0.000 & 0.001 & 0.507 & 0.000 & 0.000 & 0.169 & 0.000 & 0.001 & 0.085 \\
\hline IXX109 & 0.2546 & 2732 & 0.072 & 0.000 & 0.002 & 0.095 & 0.000 & 0.000 & 0.396 & 0.000 & 0.001 & 0.197 & 0.000 & 0.001 & 0.236 \\
\hline TX110 & 0.2251 & 1256 & 0.079 & 0.000 & 0.000 & 0.066 & 0.001 & 0.000 & 0.367 & 0.000 & 0.003 & 0.195 & 0.000 & 0.000 & 0.289 \\
\hline IX111 & 0.2599 & 2997 & 0.082 & 0.000 & 0.000 & 0.102 & 0.000 & 0.000 & 0.408 & 0.000 & 0.000 & 0.196 & 0.000 & 0.000 & 0.212 \\
\hline TX112 & 0.1543 & 716 & 0.050 & 0.000 & 0.000 & 0.049 & 0.000 & 0.000 & 0.367 & 0.000 & 0.000 & 0.178 & 0.000 & 0.002 & 0.354 \\
\hline $\mathrm{FX113}$ & 0.1702 & 8511 & 0.067 & 0.000 & 0.000 & 0.048 & 0.000 & 0,001 & 0.360 & 0.000 & 0.001 & 0.205 & 0.000 & 0.002 & 0.316 \\
\hline TX114 & 915 & 874 & 0.052 & 0.000 & 0.000 & 0.075 & 0.000 & 0.000 & 0.368 & 0.000 & 0.005 & 0.197 & 0.000 & 0.003 & 0.300 \\
\hline TX115 & 0.1577 & 7887 & 0.020 & 0.000 & 0.000 & 0.040 & 0.000 & 0.000 & 0.291 & 0.000 & 0.000 & 0.197 & 0.000 & 0.002 & 0.450 \\
\hline TX116 & 0.1611 & 3057 & 0.060 & 0.000 & 0.000 & 0.054 & 0.000 & 0.000 & 0.344 & 0.000 & 0.003 & 0.194 & 0.000 & 0.000 & 0.345 \\
\hline TX117 & 0.163 & 3152 & 0.055 & 0.000 & 0.000 & 0.068 & 0.000 & 0.000 & 0.360 & 0.000 & 0.002 & 0.194 & 0.000 & 0.001 & 0.320 \\
\hline TX118 & 0.2686 & 3432 & 0.056 & 0.000 & 0.000 & 0.079 & 0.000 & 0.001 & 0.391 & 0.000 & 0.000 & 0.208 & 0.000 & 0.004 & 0.261 \\
\hline TY102 & 0.3758 & .8789 & 0.996 & 0.000 & 0.000 & 0.004 & 0.000 & 0.000 & 0.000 & 0.000 & 0.000 & 0.000 & 0.000 & 0.000 & 0.000 \\
\hline U102 & 0.1634 & 0.8171 & 0.821 & 0.000 & 0.000 & 0.089 & 0.000 & 0.000 & 0.086 & 0.000 & 0.000 & 0.004 & 0.000 & 0.000 & 0.000 \\
\hline U103 & 0.1278 & 0.6392 & 0.804 & 0.000 & 0.000 & 0.076 & 0.000 & 0.000 & 0.109 & 0.000 & 0.000 & 0.008 & 0.000 & 0.000 & 0.003 \\
\hline U105 & 0.1468 & 0.7338 & 0.028 & 0.000 & 0.000 & 0.058 & 0.000 & 0.000 & 0.518 & 0.000 & 0.000 & 0.238 & 0.000 & 0.000 & 0.158 \\
\hline U106 & 0.2194 & 1.0972 & 0.884 & 0.000 & 0.000 & 0.067 & 0.000 & 0.000 & 0.048 & 0.000 & 0.000 & 0.001 & 0.000 & 0.000 & 0.000 \\
\hline U107 & 0.1513 & 0.7566 & 0.134 & 0.000 & 0.000 & 0.078 & 0.000 & 0.000 & 0.428 & 0.000 & 0.000 & 0.157 & 0.000 & 0.000 & 0.203 \\
\hline U108 & 0.1278 & 0.6392 & 0.107 & 0.000 & 0.000 & 0.069 & 0.000 & 0.000 & 0.430 & 0.000 & 0.000 & 0.181 & 0.000 & 0.000 & 0.213 \\
\hline U109 & 0.1297 & 0.6487 & 0.380 & 0.000 & 0.000 & 0.193 & 0.000 & 0.000 & 0.388 & 0.000 & 0.000 & 0.031 & 0.000 & 0.000 & 0.008 \\
\hline U111 & 0.1805 & 0.9023 & 0.803 & 0.000 & 0.000 & 0.121 & 0.000 & 0.000 & 0.076 & 0.000 & 0.000 & 0.000 & 0.000 & 0.000 & 0.000 \\
\hline
\end{tabular}


Table B.2. Condensed Phase Organic Reactions Safety Issue Expected Value of Perfect Information and Best Mitigation Without Information for Saltcake Tanks

\begin{tabular}{|c|c|c|c|c|}
\hline Tank & $\begin{array}{c}\text { Expected Value of } \\
\text { Perfect Information } \\
\text { (\$millions) }\end{array}$ & $\begin{array}{l}\text { Expected Value of Perfect } \\
\text { Information about TOC } \\
\text { (\$millions) }\end{array}$ & $\begin{array}{l}\text { Expected Value of Perfect } \\
\text { Information about Moisture } \\
\text { (\$ millions) }\end{array}$ & $\begin{array}{c}\text { Best Mitigation without } \\
\text { Information }\end{array}$ \\
\hline A101 & 0.007 & 0.007 & 0.000 & No Action \\
\hline $\mathrm{A} 102$ & 0.000 & 0.000 & 0.000 & No Action \\
\hline AX101 & 0.027 & 0.027 & 0.014 & No Action \\
\hline $\mathrm{AX102}$ & 0.000 & 0.000 & 0.000 & No Action \\
\hline $\mathrm{AX} 103$ & 0.000 & 0.000 & 0.000 & No Action \\
\hline B102 & 0.000 & 0.000 & 0.000 & No Action \\
\hline B104 & 0.000 & 0.000 & 0.000 & No Action \\
\hline $\mathrm{B} 105$ & 0.007 & 0.007 & 0.000 & No Action \\
\hline BX105 & 0.000 & 0.000 & 0.000 & No Action \\
\hline BX110 & 0.000 & 0.000 & 0.000 & No Action \\
\hline BX111 & 0.000 & 0.000 & 0.000 & No Action \\
\hline BY101 & 0.013 & 0.013 & 0.000 & Control ignition sources \\
\hline BY102 & 0.004 & 0.004 & 0.000 & No Action \\
\hline BY103 & 0.006 & 0.006 & 0.000 & No Action \\
\hline BY104 & 0.029 & 0.003 & 0.020 & \begin{tabular}{|l} 
Control ignition sources \\
\end{tabular} \\
\hline BY105 & 0.022 & 0.022 & 0.010 & No Action \\
\hline BY106 & 0.014 & 0.014 & 0.004 & No Action \\
\hline BY107 & 0.267 & 0.016 & 0.200 & \begin{tabular}{|c|} 
Control ignition sources \\
\end{tabular} \\
\hline BY108 & 0.054 & 0.029 & 0.020 & Control ignition sources \\
\hline BY109 & 0.007 & 0.007 & 0.000 & No Action \\
\hline BY110 & 0.137 & 0.012 & 0.100 & Control ignition sources \\
\hline BY111 & 0.435 & 0.009 & 0.340 & Control ignition sources \\
\hline BY112 & 0.220 & 0.014 & 0.163 & Control ignition sources \\
\hline$\$ 101$ & 0.143 & 0.090 & 0.080 & Control ignition sources \\
\hline $\mathrm{S} 102$ & 0.027 & 0.027 & 0.000 & Control ignition sources \\
\hline S103 & 0.045 & 0.045 & 0.000 & Control ignition sources \\
\hline S105 & 1.713 & 0.549 & 1.254 & \begin{tabular}{|c|}
$\begin{array}{c}\text { Moisture monitoring and } \\
\text { control }\end{array}$ \\
\end{tabular} \\
\hline S106 & 0.031 & 0.031 & 0.000 & Control ignition sources \\
\hline S107 & 0.041 & 0.041 & 0.013 & No Action \\
\hline$\$ 108$ & 0.026 & 0.026 & 0.000 & Control ignition sources \\
\hline S109 & 0.029 & 0.029 & 0.000 & Control ignition sources \\
\hline$\$ 110$ & 0.041 & 0.041 & 0.000 & Control ignition sources \\
\hline$\$ 111$ & 0.007 & 0.007 & 0.000 & Control ignition sources \\
\hline $\mathrm{S112}$ & 0.025 & 0.025 & 0.000 & Control ignition sources \\
\hline SX101 & 0.623 & 0.016 & 0.481 & Control ignition sources \\
\hline $\mathrm{SX} 102$ & 0.011 & 0.011 & 0.000 & Control ignition sources \\
\hline SX103 & 0.464 & 0.009 & 0.360 & Control ignition sources \\
\hline
\end{tabular}




\begin{tabular}{|c|c|c|c|c|}
\hline Tank & $\begin{array}{c}\text { Expected Value of } \\
\text { Perfect Information } \\
\text { (\$millions) }\end{array}$ & $\begin{array}{c}\text { Expected Value of Perfect } \\
\text { Information about TOC } \\
\text { (\$millions) }\end{array}$ & $\begin{array}{c}\text { Expected Value of Perfect } \\
\text { Information about Moisture } \\
(\$ \text { millions })\end{array}$ & \begin{tabular}{|c} 
Best Mitigation without \\
Information
\end{tabular} \\
\hline SX104 & 0.109 & 0.008 & 0.080 & Control ignition sources \\
\hline SX105 & 0.009 & 0.008 & 0.001 & Control ignition sources \\
\hline SX106 & 0.192 & 0.011 & 0.144 & Control ignition sources \\
\hline TX102 & 1.075 & 0.332 & 0.779 & $\begin{array}{c}\begin{array}{c}\text { Moisture monitoring and } \\
\text { control }\end{array} \\
\end{array}$ \\
\hline TX104 & 0.030 & 0.030 & 0.003 & No Action \\
\hline TX105 & 1.976 & 0.664 & 1.459 & \begin{tabular}{|c}
$\begin{array}{c}\text { Moisture monitoring and } \\
\text { control }\end{array}$ \\
\end{tabular} \\
\hline TX106 & 1.694 & 0.523 & 1.252 & \begin{tabular}{|c|}
$\begin{array}{c}\text { Moisture monitoring and } \\
\text { control }\end{array}$ \\
\end{tabular} \\
\hline TX107 & 0.006 & 0.006 & 0.000 & No Action \\
\hline TX108 & 0.125 & 0.024 & 0.080 & Control ignition sources \\
\hline TX109 & 0.142 & 0.017 & 0.099 & Control ignition sources \\
\hline TX110 & 0.093 & 0.014 & 0.063 & Control ignition sources \\
\hline TX111 & 0.018 & 0.018 & 0.000 & Control ignition sources \\
\hline TX112 & 0.210 & 0.010 & 0.160 & Control ignition sources \\
\hline TX113 & 0.238 & 0.011 & 0.179 & Control ignition sources \\
\hline TX114 & 0.439 & 0.013 & 0.339 & Control ignition sources \\
\hline TX115 & 0.206 & 0.006 & 0.160 & Control ignition sources \\
\hline TX116 & 0.087 & 0.011 & 0.061 & Control ignition sources \\
\hline TX117 & 0.163 & 0.012 & 0.119 & Control ignition sources \\
\hline TX118 & 0.416 & 0.013 & 0.320 & Control ignition sources \\
\hline TY102 & 0.000 & 0.000 & 0.000 & No Action \\
\hline $\mathrm{U} 102$ & 0.007 & 0.007 & 0.000 & No Action \\
\hline U103 & 0.024 & 0.024 & 0.014 & No Action \\
\hline $\mathrm{U} 105$ & 0.009 & 0.009 & 0.000 & Control ignition sources \\
\hline $\mathrm{U} 106$ & 0.002 & 0.002 & 0.000 & No Action \\
\hline $\mathrm{U} 107$ & 0.021 & 0.021 & 0.000 & Control ignition sources \\
\hline U108 & 0.018 & 0.018 & 0.000 & Control ignition sources \\
\hline U109 & 0.057 & 0.057 & 0.026 & Control ignition sources \\
\hline W11. & 0.001 & 0.001 & 0.000 & No Action \\
\hline
\end{tabular}




\section{Distribution}

No. of

Copies

Offsite

2 DOE Office of Scientific and Technical Information (OSTI)

1 Decision Science Associates, Inc. 10980 Poplar Ford Trail Manassas, VA 20109-2106

Attn: Jacob W. Ulvila

3 Decision Insights, Inc.

2062 Business Center Drive, Suite 110 Irvine, CA 92612

Attn: Thomas Eppel

Richard Johns

Detlof von Winterfeldt

Onsite

1 Richland Operations Office

Public Reading Room $\quad$ H2-53
No. of

Copies

29 Pacific Northwest National Laboratory

S. Q. Bennett

K7-90

G. R Bilyard

K8-03

M. E. Brewster

K1-96

A. J. Brothers

K8-07

S. D. Colson

K2-14

T. J. DeForest

K7-97

L. L. Fassbender (2)

K8-11

T. A. Ferryman

K5-12

S. W. Gajewski

K8-03

R. E. Gephart

K9-76

C. L. Glantz

K6-80

B. L. Harper

P. G. Heasler

J. G. Hill

V. L. Hunter (3)

K3-54

K5-12

K7-94

K7-97

D. M. Kashporenko

K8-18

L. G. Morgan

K6-48

P. J. Pelto

K7-97

D. A. Seaver

K8-07

R. D. Scheele

P7-25

T. W. Wood

K7-94

Information Release (5)

K1-06

Distr. 1 